TEXTS IN COMPUTATIONAL SCIENCE AND ENGINEERING

Svein Linge · Hans Petter Langtangen

\title{
Programming for \\ Computations - \\ MATLAB/Octave
}

Editorial Board

T. J. Barth M. Griebel D.E. Keyes

R. M. Nieminen

D. Roose

Springer Open

T. Schlick 


\section{Texts in Computational Science and Engineering}

Editors

Timothy J. Barth

Michael Griebel

David E. Keyes

Risto M. Nieminen

Dirk Roose

Tamar Schlick 
More information about this series at http://www.springer.com/series/5151 
Svein Linge - Hans Petter Langtangen

\section{Programming \\ for Computations \\ - MATLAB/Octave}

A Gentle Introduction to Numerical

Simulations with MATLAB/Octave

黛 Springer Open 


\section{Svein Linge}

Department of Process, Energy and Environmental Technology

University College of Southeast Norway Porsgrunn, Norway
Hans Petter Langtangen

Simula Research Laboratory

Lysaker, Norway

On leave from:

Department of Informatics

University of Oslo

Oslo, Norway

ISSN 1611-0994

Texts in Computational Science and Engineering

ISBN 978-3-319-32451-7

ISBN 978-3-319-32452-4 (eBook)

DOI 10.1007/978-3-319-32452-4

Springer Heidelberg Dordrecht London New York

Library of Congress Control Number: 2016947215

Mathematic Subject Classification (2010): 34, 35, 65, 68

(C) The Editor(s) (if applicable) and the Author(s) 2016 This book is published open access.

Open Access This book is distributed under the terms of the Creative Commons Attribution-NonCommercial 4.0 International License (http://creativecommons.org/licenses/by-nc/4.0/), which permits any noncommercial use, duplication, adaptation, distribution and reproduction in any medium or format, as long as you give appropriate credit to the original author(s) and the source, a link is provided to the Creative Commons license and any changes made are indicated.

The images or other third party material in this book are included in the work's Creative Commons license, unless indicated otherwise in the credit line; if such material is not included in the work's Creative Commons license and the respective action is not permitted by statutory regulation, users will need to obtain permission from the license holder to duplicate, adapt or reproduce the material.

This work is subject to copyright. All commercial rights are reserved by the Publisher, whether the whole or part of the material is concerned, specifically the rights of translation, reprinting, reuse of illustrations, recitation, broadcasting, reproduction on microfilms or in any other physical way, and transmission or information storage and retrieval, electronic adaptation, computer software, or by similar or dissimilar methodology now known or hereafter developed.

The use of general descriptive names, registered names, trademarks, service marks, etc. in this publication does not imply, even in the absence of a specific statement, that such names are exempt from the relevant protective laws and regulations and therefore free for general use.

The publisher, the authors and the editors are safe to assume that the advice and information in this book are believed to be true and accurate at the date of publication. Neither the publisher nor the authors or the editors give a warranty, express or implied, with respect to the material contained herein or for any errors or omissions that may have been made.

Printed on acid-free paper

Springer International Publishing AG Switzerland is part of Springer Science+Business Media (www.springer.com) 


\section{Preface}

Computing, in the sense of doing mathematical calculations, is a skill that mankind has developed over thousands of years. Programming, on the other hand, is in its infancy, with a history that spans a few decades only. Both topics are vastly comprehensive and usually taught as separate subjects in educational institutions around the world, especially at the undergraduate level. This book is about the combination of the two, because computing today becomes so much more powerful when combined with programming.

Most universities and colleges implicitly require students to specialize in computer science if they want to learn the craft of programming, since other student programs usually do not offer programming to an extent demanded for really mastering this craft. Common arguments claim that it is sufficient with a brief introduction, that there is not enough room for learning programming in addition to all other must-have subjects, and that there is so much software available that few really need to program themselves. A consequence is that engineering students often graduate with shallow knowledge about programming, unless they happened to choose the computer science direction.

We think this is an unfortunate situation. There is no doubt that practicing engineers and scientists need to know their pen and paper mathematics. They must also be able to run off-the-shelf software for important standard tasks and will certainly do that a lot. Nevertheless, the benefits of mastering programming are many.

\section{Why learn programming?}

1. Ready-made software is limited to handling certain standard problems. What do you do when the problem at hand is not covered by the software you bought? Fortunately, a lot of modern software systems are extensible via programming. In fact, many systems demand parts of the problem specification (e.g., material models) to be specified by computer code.

2. With programming skills, you may extend the flexibility of existing software packages by combining them. For example, you may integrate packages that do not speak to each other from the outset. This makes the work flow simpler, more efficient, and more reliable, and it puts you in position to attack new problems. 
3. It is easy to use excellent ready-made software the wrong way. Insight in programming and the mathematics behind is fundamental for understanding complex software, avoiding pitfalls, and become a safe user.

4. Bugs (errors in computer code) are present in most larger computer programs (also in the ones from the shop!). What do you do when your ready-made software gives unexpected results? Is it a bug, is it wrong use, or is it the mathematically correct result? Experience with programming of mathematics gives you a good background for answering these questions. The one who can program, can also make tailored code for a simplified problem setting and use that to verify the computations done with off-the-shelf software.

5. Lots of skilled people around the world solve computational problems by writing their own code and offer their code for free on the Internet. To take advantage of this truly great source of software in a reliable way, one must normally be able to understand and possibly modify computer code offered by others.

6. It is recognized world wide that students struggle with mathematics and physics. Too many find such subjects difficult and boring. With programming, we can execute the good old subjects in a brand new way! According to the authors' own experience, students find it much more motivating and enlightening when programming is made an integrated part of mathematics and physical science courses. In particular, the problem being solved can be much more realistic than when the mathematics is restricted to what you can do with pen and paper.

7. Finally, we launch our most important argument for learning computer programming: the algorithmic thinking that comes with the process of writing a program for a computational problem enforces a thorough understanding of both the problem and solution method. We can simply quote the famous Norwegian computer scientist Kristen Nyggaard: "Programming is understanding".

In the authors' experience, programming is an excellent pedagogical tool for understanding mathematics: "You think you know when you can learn, are more sure when you can write, even more when you can teach, but certain when you can program" (Alan Perlis, computer scientist, 1922-1990). Consider, for example, integration. A numerical method for integration has a much stronger focus on what the integral actually is and means compared to analytical methods, where much time and effort must be devoted to integration by parts, integration by substitution, etc. Moreover, when programming the numerical integration formula, it becomes evident that it works for "all" mathematical functions and that the implementation should be in terms of a general function applicable to "all" integrals. In this way, students learn to recognize a special problem as belonging to a class of problems (e.g., integration, differential equations, root finding), for which we have general numerical methods implemented in widely applicable software. When they write this software, as we do in this book, they learn how to generalize and increase the abstraction level of the mathematical problem. When they use this software, they learn how a special case should be attacked by general methods and software for the class of problems that comprises the special case at hand. This is the power of mathematics in a nutshell, and it is paramount that students understand this way of thinking. 
Target audience and background knowledge This book was written for students, teachers, engineers and scientists that know nothing about programming and numerical methods from before, but who seek a minimum of the fundamental skills required to get started with programming as a tool for solving scientific and engineering problems. Some knowledge of one- and multi-variable calculus is assumed. The basic programming concepts are presented in only 50 pages (Chaps. 1 and 2), before practical applications of these concepts are demonstrated in important mathematical subjects addressed in the remaining parts of the book (Chaps. 3-6). Each chapter is followed by a set of exercises that cover a wide range of application areas, e.g. biology, geology, statistics, physics and mathematics. The exercises were particularly designed to bring across important points from the text. The reader will realize that the modest content of the first 50 pages can in fact bring you quite far in powerful problem solving!

Learning the very basics of programming should not take long, but as with any other craft, mastering the skill requires continued and extensive practice. Some beginning practice is gained through Chaps. 3-6, but the authors strongly emphasize that this is only a start. Students should continue to practice programming in subsequent courses, while those who exercise self-study, should keep up the learning process through continued application of the craft. The book is a good starting point when teaching computer programming as an integrated part of standard university courses in mathematics and physical sciences. In our experience, such an integration is doable and indeed rewarding.

Numerical methods An overall goal with this book is to motivate computer programming as a very powerful tool for doing mathematics. All examples are related to mathematics and its use in engineering and science. However, to solve mathematical problems through computer programming, we need numerical methods. Explaining basic numerical methods is therefore an integral part of the book. Our choice of topics is governed by what is most needed in science and engineering, as well as in the teaching of applied physical science courses. Mathematical models are then central, with differential equations constituting the most frequent type of models. Consequently, the numerical focus in this book is on differential equations. As a soft pedagogical starter for the programming of mathematics, we have chosen the topic of numerical integration. There is also a chapter on root finding, which is important for the numerical solution of nonlinear differential equations. We remark that the book is deliberately brief on numerical methods. This is because our focus is on implementing numerical algorithms, but to develop reliable, working programs, the programmer must be confident about the basic ideas of the numerical approximations involved.

The computer language: Matlab We have chosen to use the programming language Matlab, because this language gives very compact and readable code that closely resembles the mathematical recipe for solving the problem at hand. Matlab also has a gentle learning curve. There is a Python companion of this book in case that language is preferred. Comparing these two versions of the book provides an excellent demonstration of how similar these languages are. We use the term Matlab throughout this book to mean the commercial MATLAB (R) software [12] or the open source alternative Octave [4]. Other computer languages, like Fortran, 
$\mathrm{C}$, and $\mathrm{C}++$, have a strong position in science and engineering. During the last two decades, however, there has been a significant shift in popularity from these compiled languages to more high-level and easier-to-read languages like Matlab, Python, R, Maple, Mathematica, and IDL, for instance. This latter class of languages is computationally less efficient, but superior with respect to overall human problem solving efficiency. This book emphasizes how to think like a programmer, rather than focusing on technical language details. Thus, the book should put the reader in a good position for learning other programming languages later, including the classic ones: Fortran, $\mathrm{C}$, and $\mathrm{C}++$.

How this book is different There are numerous texts on computer programming and numerical methods, so how does the present one differ from the existing literature? Compared to books on numerical methods, our book has a much stronger emphasis on the craft of programming and on verification. We want to give students a thorough understanding of how one thinks about programming as a problem solving method and how one can be sure that programs are correct (well, you can never be completely sure, but we show how you can provide convincing evidence for correctness).

Even though there are lots of books on numerical methods where many algorithms have a corresponding computer implementation (see, e.g., [1-3, 5-7, 11, 13-22]) it is assumed that the reader "can program" beforehand. The present book teaches the craft of structured programming along with the fundamental ideas of numerical methods. Furthermore, we have so far not found any other numerical methods book that has a strong emphasis on verifying implementations. In this book, unit testing and corresponding test functions are introduced early on. We also put much emphasis on coding algorithms as functions, as opposed to "flat programs", which often dominate in the literature and among practitioners. Functions are reusable because they utilize the general formulation of a mathematical algorithm such that it becomes applicable to a large class of problems.

There are also numerous books on computer programming, but to our knowledge only one [9] that aims to teach how to think about programming in the context of numerical methods and scientific applications. That book [9] has its primary focus on teaching Python and is a very comprehensive introduction to Python as a language and the thinking about programming as a computer scientist. Sometimes one needs a text that does not go so deep into the language-specific details, but instead targets the shortest path to reliable mathematical problem solving through programming. With this attitude in mind, a lot of topics were left out of the present book, simply because they were not strictly needed in the mathematical problem solving process. An example of such a topic is object-oriented programming.

Whenever the need for a structured introduction to programming arises in science and engineering courses, this book may be your option, either for self-study or for use in organized teaching. The thinking, habits, and practice covered in a couple of hundred pages will put readers in a firm position for utilizing and understanding the power of computers for problem solving in science and engineering.

Supplementary materials All program and data files referred to in this book are available from the book's primary web site: http://hplgit.github.io/prog4comp/. 
Acknowledgments First of all, we want to thank all students who attended the courses FM1006 Modelling and simulation of dynamic systems, FM1115 Scientific Computing, FB1012 Mathematics I and FB2112 Physics at the University College of Southeast Norway over the last couple of years. They worked their way through early versions of this text and gave us constructive and positive feedback that helped us correct errors and improve the book in so many ways. Special acknowledgement goes to Guandong Kou and Edirisinghe V. P. J. Manjula for their careful reading of the manuscript and constructive suggestions for improvement. The careful proof reading by Yapi Donatien Achou is also highly appreciated. We thank all our good colleagues at the University College of Southeast Norway, the University of Oslo, and Simula Research Laboratory for their continued support and interest, enlightening discussions, and for providing such an inspiring environment for teaching and science. In particular, Svein Linge is thankful to Marius Lysaker for their fruitful collaboration on introducing programming as an integral part of mathematics and physics bachelor courses at the University College of Southeast Norway. Finally, the authors must thank the Springer team with Dr. Martin Peters, Thanh-Ha Le Thi, and Yvonne Schlatter for the effective editorial and production process.

The text was written in the DocOnce ${ }^{1}$ [8] markup language, which allowed us to work with a single text source for both the Python and the Matlab version of this book, and to produce various electronic versions of the book.

December 2015

Svein Linge and Hans Petter Langtangen

\footnotetext{
${ }^{1}$ https://github.com/hplgit/doconce
} 


\section{Contents}

1 The First Few Steps . . . . . . . . . . . . . . . . . 1

1.1 What Is a Program? And What Is Programming? . . . . . . . . . 1

1.2 A Matlab Program with Variables ... . . . . . . . . . 3

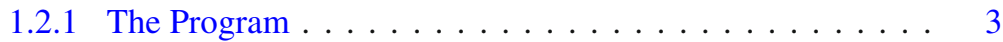

1.2.2 Dissection of the Program . . . . . . . . . . . 4

1.2.3 Why Not Just Use a Pocket Calculator? . . . . . . . . . 5

1.2.4 Why You Must Use a Text Editor to Write Programs . . . . 6

1.2.5 Write and Run Your First Program . . . . . . . . . . . 6

1.3 A Matlab Program with a Library Function . . . . . . . . . . . 7

1.4 A Matlab Program with Vectorization and Plotting . . . . . . . . 8

1.5 More Basic Concepts . . . . . . . . . . . . . . . . . . . . . 10

1.5.1 Using Matlab Interactively . . . . . . . . . . . . . . 10

1.5.2 Arithmetics, Parentheses and Rounding Errors . . . . . . . . 11

1.5 .3 Variables . . . . . . . . . . . . . . . . . 11

1.5.4 Formatting Text and Numbers . . . . . . . . . . . . . . 12

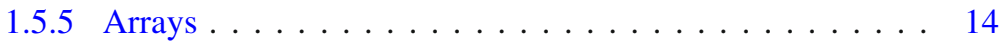

1.5 .6 Plotting ............................. 15

1.5.7 Error Messages and Warnings . . . . . . . . . . . . 18

1.5 .8 Input Data . . . . . . . . . . . . . . . . . 19

1.5.9 Symbolic Computations . . . . . . . . . . . . . . 19

1.5.10 Concluding Remarks . . . . . . . . . . . . . . . . 21

1.6 Exercises ....................... 22

2 Basic Constructions . . . . . . . . . . . . . . . . . . . 25

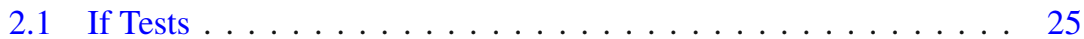

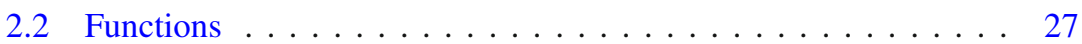

2.3 For Loops . . . . . . . . . . . . . . . . . . . . . . . . . . . . . . . . . . . . . 32

2.4 While Loops . . . . . . . . . . . . . . . . . . . 35

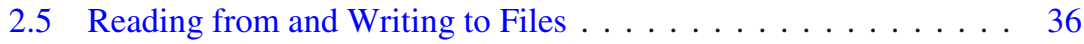

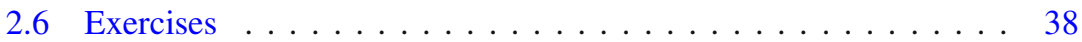

$3 \quad$ Computing Integrals $\ldots \ldots \ldots \ldots \ldots \ldots$. . . . . . . . . . . . . . . . 47

3.1 Basic Ideas of Numerical Integration $\ldots \ldots \ldots \ldots . \ldots . . \ldots 48$

3.2 The Composite Trapezoidal Rule . . . . . . . . . . . . . . . . . . . 49 
3.2.1 The General Formula . . . . . . . . . . . . . . . . . 51

3.2 .2 Implementation . . . . . . . . . . . . . . . . 52

3.2.3 Alternative Flat Special-Purpose Implementation . . . . . . 54

3.3 The Composite Midpoint Method . . . . . . . . . . . . . . . 57

3.3.1 The General Formula . . . . . . . . . . . . . . . . . . 58

3.3 .2 Implementation . . . . . . . . . . . . . 58

3.3.3 Comparing the Trapezoidal and the Midpoint Methods . . . 59

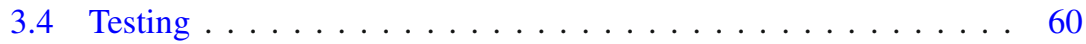

3.4.1 Problems with Brief Testing Procedures . . . . . . . . . 60

3.4 .2 Proper Test Procedures . . . . . . . . . . . . 61

3.4.3 Finite Precision of Floating-Point Numbers . . . . . . . . . 62

3.4.4 Constructing Unit Tests and Writing Test Functions . . . . . 64

3.5 Vectorization . . . . . . . . . . . . . . . . 67

3.6 Measuring Computational Speed . . . . . . . . . . . . . . . . . . . . 69

3.7 Double and Triple Integrals . . . . . . . . . . . . . . . . . . 69

3.7.1 The Midpoint Rule for a Double Integral . . . . . . . . . 69

3.7.2 The Midpoint Rule for a Triple Integral . . . . . . . . . . 73

3.7.3 Monte Carlo Integration for Complex-Shaped Domains . . 76

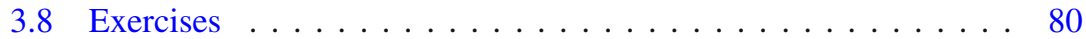

4 Solving Ordinary Differential Equations . . . . . . . . . . . . . . . . 87

4.1 Population Growth $\ldots \ldots \ldots \ldots \ldots$

4.1.1 Derivation of the Model . . . . . . . . . . . . . . . . 89

4.1 .2 Numerical Solution . . . . . . . . . . . . . . . . . . . . 91

4.1.3 Programming the Forward Euler Scheme; the Special Case 94

4.1.4 Understanding the Forward Euler Method . . . . . . . . . . 97

4.1.5 Programming the Forward Euler Scheme; the General Case 97

4.1.6 Making the Population Growth Model More Realistic . . . 98

4.1.7 Verification: Exact Linear Solution of the Discrete Equations 101

4.2 Spreading of Diseases . . . . . . . . . . . . . . . . . . 102

4.2 .1 Spreading of a Flu . . . . . . . . . . . . . 102

4.2.2 A Forward Euler Method for the Differential Equation

System . . . . . . . . . . . . . . . 105

4.2.3 Programming the Numerical Method; the Special Case . . 105

4.2 .4 Outbreak or Not . . . . . . . . . . . . . . . . . . 106

4.2.5 Abstract Problem and Notation . . . . . . . . . . . . . 108

4.2.6 Programming the Numerical Method; the General Case . . 109

4.2 .7 Time-Restricted Immunity . . . . . . . . . . . . . . 111

4.2 .8 Incorporating Vaccination . . . . . . . . . . . . . . . 111

4.2.9 Discontinuous Coefficients: a Vaccination Campaign . . . . 114

4.3 Oscillating One-Dimensional Systems . . . . . . . . . . . . . 115

4.3.1 Derivation of a Simple Model . . . . . . . . . . . . 115

4.3.2 Numerical Solution . . . . . . . . . . . . . . . . . . 117

4.3.3 Programming the Numerical Method; the Special Case . . . 117

4.3.4 A Magic Fix of the Numerical Method . . . . . . . . . . 120

4.3.5 The 2nd-Order Runge-Kutta Method (or Heun's Method) . 122

4.3.6 Software for Solving ODEs . . . . . . . . . . . . . . . . 123

4.3.7 The 4th-Order Runge-Kutta Method . . . . . . . . . . 130 
4.3.8 More Effects: Damping, Nonlinearity, and External Forces 133

4.3.9 Illustration of Linear Damping . . . . . . . . . . . . . 136

4.3.10 Illustration of Linear Damping with Sinusoidal Excitation . 137

4.3.11 Spring-Mass System with Sliding Friction . . . . . . . . . 138

4.3.12 A Finite Difference Method; Undamped, Linear Case . . . 141

4.3.13 A Finite Difference Method; Linear Damping . . . . . . . 143

4.4 Exercises . . . . . . . . . . . . . . . . . . . . . . 144

$5 \quad$ Solving Partial Differential Equations . . . . . . . . . . . . . . . . 153

5.1 Finite Difference Methods . . . . . . . . . . . . . . . . . 155

5.1.1 Reduction of a PDE to a System of ODEs . . . . . . . . 156

5.1.2 Construction of a Test Problem with Known Discrete

Solution . . . . . . . . . . . . . . . . 158

5.1.3 Implementation: Forward Euler Method . . . . . . . . . . . 158

5.1.4 Application: Heat Conduction in a Rod . . . . . . . . . 160

5.1 .5 Vectorization . . . . . . . . . . . . . . . 165

5.1.6 Using Odespy to Solve the System of ODEs . . . . . . . 165

5.1 .7 Implicit Methods . . . . . . . . . . . . . . . . . . . 166

5.2 Exercises . . . . . . . . . . . . . . . . . . 169

6 Solving Nonlinear Algebraic Equations . . . . . . . . . . . . . . . . . 177

6.1 Brute Force Methods . . . . . . . . . . . . . . . . . . . . 178

6.1 .1 Brute Force Root Finding . . . . . . . . . . . . . . . . . . 179

6.1 .2 Brute Force Optimization . . . . . . . . . . . . . 181

6.1.3 Model Problem for Algebraic Equations . . . . . . . . . . . 182

6.2 Newton's Method . . . . . . . . . . . . . . . . . . . . 183

6.2.1 Deriving and Implementing Newton's Method . . . . . . . . 183

6.2.2 Making a More Efficient and Robust Implementation . . . . 186

6.3 The Secant Method . . . . . . . . . . . . . . . . . . . . . . . . 189

6.4 The Bisection Method . . . . . . . . . . . . . . . . . . . . . . . . . . . . . . . . . 191

6.5 Rate of Convergence . . . . . . . . . . . . . . . . . . 193

6.6 Solving Multiple Nonlinear Algebraic Equations . . . . . . . . . . . . . 196

6.6 .1 Abstract Notation . . . . . . . . . . . . . . . . . . 196

6.6.2 Taylor Expansions for Multi-Variable Functions . . . . . . 196

6.6.3 Newton's Method . . . . . . . . . . . . . . . . . . . 197

6.6 .4 Implementation . . . . . . . . . . . . . . . . . . . . . . . . . . . . . . . . . . . . . . . . .

6.7 Exercises . . . . . . . . . . . . . . . . . . . . . . . . 199

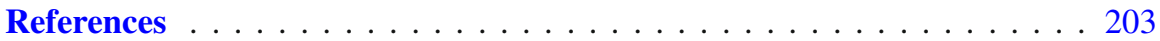

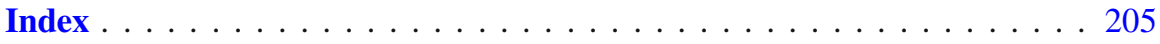




\section{List of Exercises}

Exercise 1.1: Error messages . . . . . . . . . . . . . . . . 22

Exercise 1.2: Volume of a cube . . . . . . . . . . . . . . 23

Exercise 1.3: Area and circumference of a circle . . . . . . . . . . 23

Exercise 1.4: Volumes of three cubes . . . . . . . . . . . . 23

Exercise 1.5: Average of integers . . . . . . . . . . . . . . 23

Exercise 1.6: Interactive computing of volume and area . . . . . . . . . . 23

Exercise 1.7: Update variable at command prompt . . . . . . . . . . . . 24

Exercise 1.8: Formatted print to screen . . . . . . . . . . . . . . . 24

Exercise 1.9: Matlab documentation and random numbers . . . . . . . . . 24

Exercise 2.1: Introducing errors . . . . . . . . . . . . . . . . . . . . . . . . . 38

Exercise 2.2: Compare integers $\mathrm{a}$ and $\mathrm{b} \ldots \ldots \ldots \ldots \ldots$

Exercise 2.3: Functions for circumference and area of a circle . . . . . . . 38

Exercise 2.4: Function for area of a rectangle . . . . . . . . . . . . . 39

Exercise 2.5: Area of a polygon . . . . . . . . . . . . . . . . 39

Exercise 2.6: Average of integers . . . . . . . . . . . . . . . . 40

Exercise 2.7: While loop with errors . . . . . . . . . . . . . . . . 40

Exercise 2.8: Area of rectangle versus circle . . . . . . . . . . . . . 40

Exercise 2.9: Find crossing points of two graphs . . . . . . . . . . 40

Exercise 2.10: Sort array with numbers . . . . . . . . . . . . . . . 41

Exercise 2.11: Compute $\pi \ldots \ldots \ldots \ldots$. . . . . . . . . . 41

Exercise 2.12: Compute combinations of sets . . . . . . . . . . . 41

Exercise 2.13: Frequency of random numbers . . . . . . . . . . . . . 42

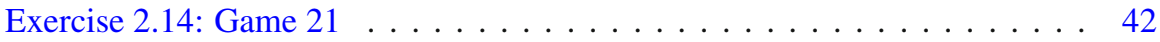

Exercise 2.15: Linear interpolation . . . . . . . . . . . . . . . . . 42

Exercise 2.16: Test straight line requirement . . . . . . . . . . . . . . 43

Exercise 2.17: Fit straight line to data . . . . . . . . . . . . . . . . 43

Exercise 2.18: Fit sines to straight line . . . . . . . . . . . . . 44

Exercise 2.19: Count occurrences of a string in a string . . . . . . . . . . 45

Exercise 3.1: Hand calculations for the trapezoidal method . . . . . . . . . . 80

Exercise 3.2: Hand calculations for the midpoint method . . . . . . . . . . 80

Exercise 3.3: Compute a simple integral . . . . . . . . . . . . . 80

Exercise 3.4: Hand-calculations with sine integrals . . . . . . . . . . . . . . 80

Exercise 3.5: Make test functions for the midpoint method . . . . . . . . . . 81

Exercise 3.6: Explore rounding errors with large numbers . . . . . . . . 81 
Exercise 3.7: Write test functions for $\int_{0}^{4} \sqrt{x} d x \ldots \ldots \ldots$. . . . . . . . . . 81

Exercise 3.8: Rectangle methods . . . . . . . . . . . . . . . . . 81

Exercise 3.9: Adaptive integration . . . . . . . . . . . . . . . 82

Exercise 3.10: Integrating $x$ raised to $x \ldots \ldots \ldots \ldots \ldots$

Exercise 3.11: Integrate products of sine functions . . . . . . . . . . . . 83

Exercise 3.12: Revisit fit of sines to a function . . . . . . . . . . 83

Exercise 3.13: Derive the trapezoidal rule for a double integral . . . . . . . . 84

Exercise 3.14: Compute the area of a triangle by Monte Carlo integration . . . 84

Exercise 4.1: Geometric construction of the Forward Euler method . . . . . . . 144

Exercise 4.2: Make test functions for the Forward Euler method . . . . . . . 145

Exercise 4.3: Implement and evaluate Heun's method . . . . . . . . . . . . . . . . 145

Exercise 4.4: Find an appropriate time step; logistic model . . . . . . . . . . . . 145

Exercise 4.5: Find an appropriate time step; SIR model . . . . . . . . . . . . . . . . 146

Exercise 4.6: Model an adaptive vaccination campaign . . . . . . . . . . . 146

Exercise 4.7: Make a SIRV model with time-limited effect of vaccination . . 146

Exercise 4.8: Refactor a flat program . . . . . . . . . . . . . . 146

Exercise 4.9: Simulate oscillations by a general ODE solver . . . . . . . . 146

Exercise 4.10: Compute the energy in oscillations . . . . . . . . . . . . . 147

Exercise 4.11: Use a Backward Euler scheme for population growth . . . . . . 147

Exercise 4.12: Use a Crank-Nicolson scheme for population growth . . . . . 148

Exercise 4.13: Understand finite differences via Taylor series . . . . . . . . . . . 148

Exercise 4.14: Use a Backward Euler scheme for oscillations . . . . . . . . . 149

Exercise 4.15: Use Heun's method for the SIR model . . . . . . . . . . . . . 150

Exercise 4.16: Use Odespy to solve a simple ODE . . . . . . . . . . . . . . 150

Exercise 4.17: Set up a Backward Euler scheme for oscillations . . . . . . . 150

Exercise 4.18: Set up a Forward Euler scheme for nonlinear and damped

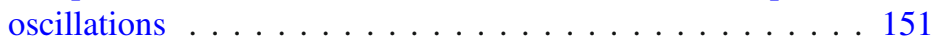

Exercise 4.19: Discretize an initial condition . . . . . . . . . . . . 151

Exercise 5.1: Simulate a diffusion equation by hand . . . . . . . . . . . . 169

Exercise 5.2: Compute temperature variations in the ground . . . . . . . 170

Exercise 5.3: Compare implicit methods . . . . . . . . . . . . . . 170

Exercise 5.4: Explore adaptive and implicit methods . . . . . . . . . . . . 171

Exercise 5.5: Investigate the $\theta$ rule . . . . . . . . . . . . . . . 171

Exercise 5.6: Compute the diffusion of a Gaussian peak . . . . . . . . . . 172

Exercise 5.7: Vectorize a function for computing the area of a polygon . . . 173

Exercise 5.8: Explore symmetry . . . . . . . . . . . . . . . . . . . . . 173

Exercise 5.9: Compute solutions as $t \rightarrow \infty \ldots \ldots \ldots \ldots \ldots \ldots$

Exercise 5.10: Solve a two-point boundary value problem . . . . . . . . . 175

Exercise 6.1: Understand why Newton's method can fail . . . . . . . . . . . 199

Exercise 6.2: See if the secant method fails . . . . . . . . . . . . . . . . 199

Exercise 6.3: Understand why the bisection method cannot fail . . . . . . . 200

Exercise 6.4: Combine the bisection method with Newton's method . . . . . 200

Exercise 6.5: Write a test function for Newton's method . . . . . . . . . . . 200

Exercise 6.6: Solve nonlinear equation for a vibrating beam . . . . . . . . 200 


\section{The First Few Steps}

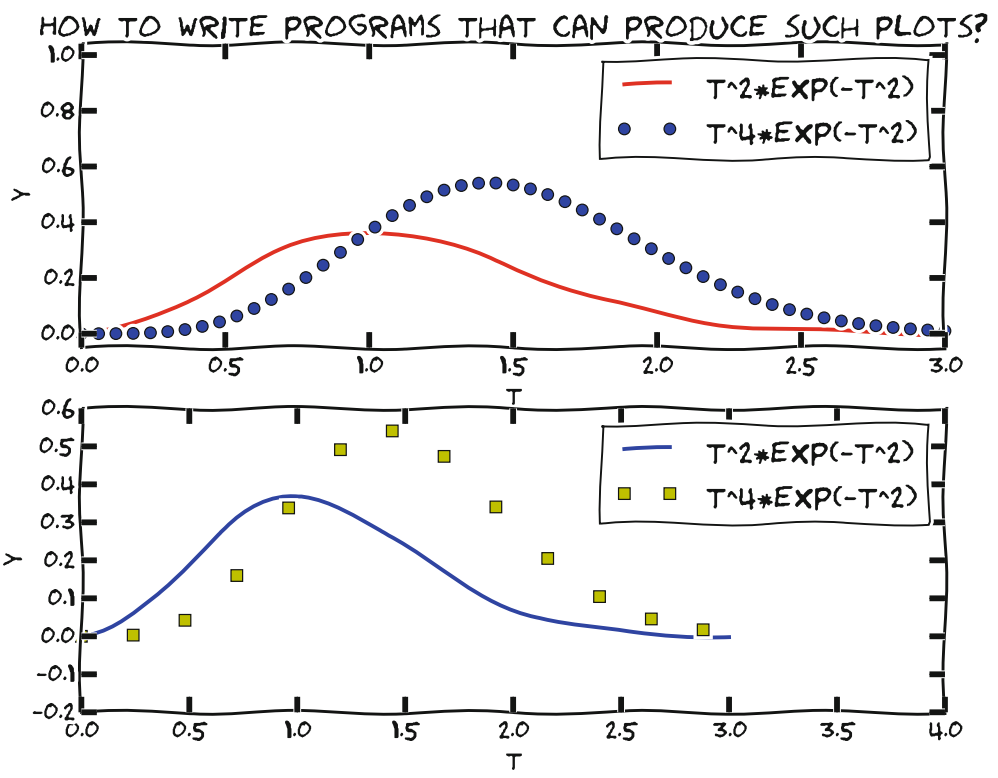

\subsection{What Is a Program? And What Is Programming?}

Today, most people are experienced with computer programs, typically programs such as Word, Excel, PowerPoint, Internet Explorer, and Photoshop. The interaction with such programs is usually quite simple and intuitive: you click on buttons, pull down menus and select operations, drag visual elements into locations, and so forth. The possible operations you can do in these programs can be combined in seemingly an infinite number of ways, only limited by your creativity and imagination.

Nevertheless, programs often make us frustrated when they cannot do what we wish. One typical situation might be the following. Say you have some measurements from a device, and the data are stored in a file with a specific format. You may want to analyze these data in Excel and make some graphics out of it. However, assume there is no menu in Excel that allows you to import data in this specific 
format. Excel can work with many different data formats, but not this one. You start searching for alternatives to Excel that can do the same and read this type of data files. Maybe you cannot find any ready-made program directly applicable. You have reached the point where knowing how to write programs on your own would be of great help to you! With some programming skills, you may write your own little program which can translate one data format to another. With that little piece of tailored code, your data may be read and analyzed, perhaps in Excel, or perhaps by a new program tailored to the computations that the measurement data demand.

The real power of computers can only be utilized if you can program them. By programming you can get the computer to do (most often!) exactly what you want. Programming consists of writing a set of instructions in a very specialized language that has adopted words and expressions from English. Such languages are known as programming or computer languages. The set of instructions is given to a program which can translate the meaning of the instructions into real actions inside the computer.

The purpose of this book is to teach you to write such instructions dedicated to solve mathematical and engineering problems by fundamental numerical methods.

There are numerous computer languages for different purposes. Within the engineering area, the most widely used computer languages are Python, MATLAB, Octave, Fortran, $\mathrm{C}, \mathrm{C}++$, and to some extent Maple, and Mathematica. How you write the instructions (i.e. the syntax) differs between the languages. Let us use an analogy.

Assume you are an international kind of person, having friends abroad in England, Russia and China. They want to try your favorite cake. What can you do? Well, you may write down the recipe in those three languages and send them over. Now, if you have been able to think correctly when writing down the recipe, and you have written the explanations according to the rules in each language, each of your friends will produce the same cake. Your recipe is the "computer program", while English, Russian and Chinese represent the "computer languages" with their own rules of how to write things. The end product, though, is still the same cake. Note that you may unintentionally introduce errors in your "recipe". Depending on the error, this may cause "baking execution" to stop, or perhaps produce the wrong cake. In your computer program, the errors you introduce are called bugs (yes, small insects! ... for historical reasons), and the process of fixing them is called debugging. When you try to run your program that contains errors, you usually get warnings or error messages. However, the response you get depends on the error and the programming language. You may even get no response, but simply the wrong "cake". Note that the rules of a programming language have to be followed very strictly. This differs from languages like English etc., where the meaning might be understood even with spelling errors and "slang" included.

This book comes in two versions, one that is based on Python, and one based on Matlab. Both Python and Matlab represent excellent programming environments for scientific and engineering tasks. The version you are reading now, is the Matlab version.

Readers who want to expand their scientific programming skills beyond the introductory level of the present exposition, are encouraged to study the book A Primer on Scientific Programming with Python [9]. This comprehensive book is as suitable for beginners as for professional programmers, and teaches the art 
of programming through a huge collection of dedicated examples. This book is considered the primary reference, and a natural extension, of the programming matters in the present book.

\section{Some computer science terms}

Note that, quite often, the terms script and scripting are used as synonyms for program and programming, respectively.

The inventor of the Perl programming language, Larry Wall, tried to explain the difference between script and program in a humorous way (from perl.com ${ }^{1}$ ): Suppose you went back to Ada Lovelace ${ }^{2}$ and asked her the difference between a script and a program. She'd probably look at you funny, then say something like: Well, a script is what you give the actors, but a program is what you give the audience. That Ada was one sharp lady... Since her time, we seem to have gotten a bit more confused about what we mean when we say scripting. It confuses even me, and I'm supposed to be one of the experts.

There are many other widely used computer science terms to pick up. Writing a program (or script or code) is often expressed as implementing the program. Executing a program means running the program. An algorithm is a recipe for how to construct a program. A bug is an error in a program, and the art of tracking down and removing bugs is called debugging (see, e.g., Wikipedia ${ }^{3}$ ). Simulating or simulation refers to using a program to mimic processes in the real world, often through solving differential equations that govern the physics of the processes.

\subsection{A Matlab Program with Variables}

Our first example regards programming a mathematical model that predicts the position of a ball thrown up in the air. From Newton's 2nd law, and by assuming negligible air resistance, one can derive a mathematical model that predicts the vertical position $y$ of the ball at time $t$. From the model one gets the formula

$$
y=v_{0} t-0.5 g t^{2},
$$

where $v_{0}$ is the initial upwards velocity and $g$ is the acceleration of gravity, for which $9.81 \mathrm{~m} \mathrm{~s}^{-2}$ is a reasonable value (even if it depends on things like location on the earth). With this formula at hand, and when $v_{0}$ is known, you may plug in a value for time and get out the corresponding height.

\subsubsection{The Program}

Let us next look at a Matlab program for evaluating this simple formula. Assume the program is contained as text in a file named ball.m. The text looks as follows (file ball.m):

\footnotetext{
${ }^{1}$ http://www.perl.com/pub/2007/12/06/soto-11.html

${ }^{2} \mathrm{http} / / /$ en.wikipedia.org/wiki/Ada_Lovelace

${ }^{3}$ http://en.wikipedia.org/wiki/Software_bug\#Etymology
} 


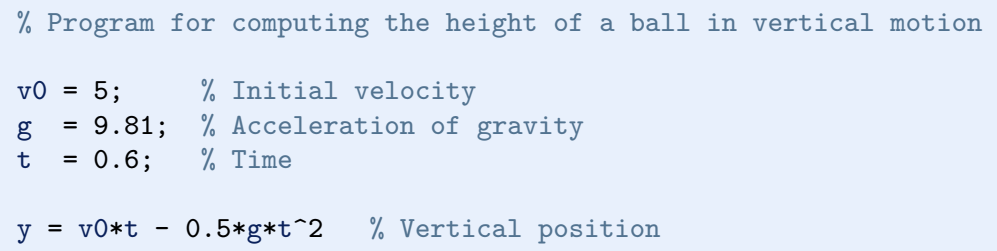

Computer programs and parts of programs are typeset with a blue background in this book. A slightly darker top and bottom bar, as above, indicates that the code is a complete program that can be run as it stands. Without the bars, the code is just a snippet and will (normally) need additional lines to run properly.

\subsubsection{Dissection of the Program}

A computer program is plain text, as here in the file ball.m, which contains instructions to the computer. Humans can read the code and understand what the program is capable of doing, but the program itself does not trigger any actions on a computer before another program, the Matlab interpreter, reads the program text and translates this text into specific actions.

\section{You must learn to play the role of a computer}

Although Matlab is responsible for reading and understanding your program, it is of fundamental importance that you fully understand the program yourself. You have to know the implication of every instruction in the program and be able to figure out the consequences of the instructions. In other words, you must be able to play the role of a computer. The reason for this strong demand of knowledge is that errors unavoidably, and quite often, will be committed in the program text, and to track down these errors, you have to simulate what the computer does with the program. Next, we shall explain all the text in ball.m in full detail.

When you run your program in Matlab, it will interpret the text in your file line by line, from the top, reading each line from left to right. The first line it reads is

\section{$\%$ Program for computing the height of a ball in vertical motion.}

This line is what we call a comment. That is, the line is not meant for Matlab to read and execute, but rather for a human that reads the code and tries to understand what is going on. Therefore, one rule in Matlab says that whenever Matlab encounters the sign $\%$ it takes the rest of the line as a comment. Matlab then simply skips reading the rest of the line and jumps to the next line. In the code, you see several such comments and probably realize that they make it easier for you to understand (or guess) what is meant with the code. In simple cases, comments are probably not much needed, but will soon be justified as the level of complexity steps up.

The next line read by Matlab is 
According to its rules, Matlab will now create a variable with the name v0 and set (the value of) that variable equal to 5 . We say that 5 is assigned to v0. This means that whenever Matlab reads v0 hereafter, it plugs in 5 instead of the name $\mathrm{v} 0$, since it knows that $\mathrm{v} 0$ has the value 5. You may think of $\mathrm{v} 0$ as a variable $v_{0}$ in mathematics. The next two lines

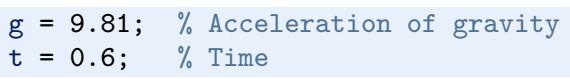

are of the same kind, so having read them too, Matlab knows of three variables (v0, $g, t)$ and their values. These variables are then used by Matlab when it reads the next line, the actual "formula",

$$
\mathrm{y}=\mathrm{v} 0 * \mathrm{t}-0.5 * \mathrm{~g} * \mathrm{t}^{-2} \quad \% \text { Vertical position }
$$

Again, according to its rules, Matlab interprets $*$ as multiplication, - as minus and - as exponent (let us also add here that, not surprisingly, + and / would have been understood as addition and division, if such signs had been present in the expression). Having read the line, Matlab performs the mathematics on the right-hand side, and then assigns the result (in this case the number 1.2342) to the variable name y. Also, since the final line has no semi-colon, Matlab understands that we also want the result printed to screen. When ball.m is run, the number 1.2342 appears on the screen.

Note that leaving out a semi-colon provides an easy way to print things to screen in general. Simply writing, e.g., v0 in the program above, i.e. without the semicolon, will make the content of v0 be printed to screen.

In the code above, you see several blank lines too. These are simply skipped by Matlab and you may use as many as you want to make a nice and readable layout of the code.

\subsubsection{Why Not Just Use a Pocket Calculator?}

Certainly, finding the answer as done by the program above could easily have been done with a pocket calculator. No objections to that and no programming would have been needed. However, what if you would like to have the position of the ball for every milli-second of the flight? All that punching on the calculator would have taken you something like four hours! If you know how to program, however, you could modify the code above slightly, using a minute or two of writing, and easily get all the positions computed in one go within a second. A much stronger argument, however, is that mathematical models from real life are often complicated and comprehensive. The pocket calculator cannot cope with such problems, even not the programmable ones, because their computational power and their programming tools are far too weak compared to what a real computer can offer. 


\subsubsection{Why You Must Use a Text Editor to Write Programs}

When Matlab interprets some code in a file, it is concerned with every character in the file, exactly as it was typed in. This makes it troublesome to write the code into a file with word processors like, e.g., Microsoft Word, since such a program will insert extra characters, invisible to us, with information on how to format the text (e.g., the font size and type). Such extra information is necessary for the text to be nicely formatted for the human eye. Matlab, however, will be much annoyed by the extra characters in the file inserted by a word processor. Therefore, it is fundamental that you write your program in a text editor where what you type on the keyboard is exactly the characters that appear in the file and that Matlab will later read. There are many text editors around. Some are stand-alone programs like Emacs, Vim, Gedit, Notepad++, and TextWrangler. Many prefer to use the text editor that comes with the graphical Matlab environment.

\subsubsection{Write and Run Your First Program}

Reading only does not teach you computer programming: you have to program yourself and practice heavily before you master mathematical problem solving via programming. Therefore, it is crucial at this stage that you write and run a Matlab program. We just went through the program ball.m above, so let us next write and run that code.

But first a warning: there are many things that must come together in the right way for ball.m to run correctly on your computer. There might be problems with your Matlab installation, with your writing of the program (it is very easy to introduce errors!), or with the location of the file, just to mention some of the most common difficulties for beginners. Fortunately, such problems are solvable, and if you do not understand how to fix the problem, ask somebody. Typically, once you are beyond these common start-up problems, you can move on to learn programming and how programs can do a lot of otherwise complicated mathematics for you.

The term Matlab refers to both the software package Matlab from MathWorks Inc., and the programming language Matlab. Matlab programs can either be run in the commercial Matlab software package, or they can be run in the free GNU Octave $^{4}$ software, usually just called Octave. We first describe how to operate the Matlab software and then Octave.

The first step is to generate a directory in which you will place your future Matlab code. Do this in a terminal window (Terminal on Mac, Power Shell or Command Prompt on Windows, or (e.g.) gnome-terminal on Linux). Write mkdir mycode to create a directory with name mycode. Then move into that directory by writing $\mathrm{cd}$ mycode.

Write and run a program in Matlab. Start Matlab and try out the following.

1. Write the Matlab program ball.m. Do this by choosing File/New/Script from the menu in the Command window. In the editor window that pops up, simply

\footnotetext{
${ }^{4}$ http://www.gnu.org/software/octave/
} 
write the code lines there as they were given above for ball.m. Now save this with the name ball.m in the right directory, i.e. myCode, via Save As from the File menu. The program is now ready for use!

2. Run the program. Do this in the Command window by writing the name of the program without the extension, i.e. write "ball", and press enter. Matlab will now run the program.

Write a program in a text editor and run it in Octave. Octave users must write the program in a plain text editor such as Gedit on Linux computers; TextWrangler on Mac, or Notepad++ on Windows. Popular, but more advanced text editors, primarily Emacs and Vim, are also available for these platforms.

1. Write the Matlab program ball . $m$ by launching a text editor and write each line exactly as they are listed in the ball.m program. Save the file as ball.m in the mycode directory.

2. Run the program. Type octave. The Octave program is started and gives you a prompt octave:1>, which indicates that you can give Octave commands. Type run ball.m and press enter. Octave will now run the program.

With a little luck, you should now get the number 1.2342 out in the command window. If so, congratulations! You have just executed your first self-written computer program in Matlab (or Octave), and you are ready to go on studying this book!

\section{m-files}

A program such as ball.m, i.e., code stored in a file with the extension .m, is usually referred to as an $m$-file.

\subsection{A Matlab Program with a Library Function}

Imagine you stand on a distance, say $10 \mathrm{~m}$ away, watching someone throwing a ball upwards. A straight line from you to the ball will then make an angle with the horizontal that increases and decreases as the ball goes up and down. Let us consider the ball at a particular moment in time, at which it has a height of $10 \mathrm{~m}$.

What is the angle of the line then? Again, this could easily be done with a calculator, but we continue to address gentle mathematical problems when learning to program. Before thinking of writing a program, one should always formulate the algorithm, i.e., the recipe for what kind of calculations that must be performed. Here, if the ball is $x \mathrm{~m}$ away and $y \mathrm{~m}$ up in the air, it makes an angle $\theta$ with the ground, where $\tan \theta=y / x$. The angle is then $\tan ^{-1}(y / x)$.

Let us make a Matlab program for doing these calculations. We introduce names $\mathrm{x}$ and $\mathrm{y}$ for the position data $x$ and $y$, and the descriptive name angle for the angle $\theta$. The program is stored in a file ball_angle.m:

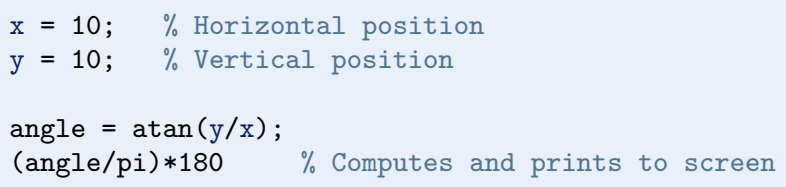


Before we turn our attention to the running of this program, let us take a look at one new thing in the code. The line angle $=\operatorname{atan}(y / x)$, illustrates how the function atan, corresponding to $\tan ^{-1}$ in mathematics, is called with the ratio $\mathrm{y} / \mathrm{x}$ as input parameter or argument. The atan function takes one argument, and the computed value is returned from atan. This means that where we see atan $(y / x)$, a computation is performed $\left(\tan ^{-1}(y / x)\right)$ and the result "replaces" the text $\operatorname{atan}(y / x)$. This is actually no more magic than if we had written just $y / x$ : then the computation of $y / x$ would take place, and the result of that division would replace the text $y / x$. Thereafter, the result is assigned to the name angle on the left-hand side of $=$.

Note that the trigonometric functions, such as atan, work with angles in radians. The return value of atan must hence be converted to degrees, and that is why we perform the computation (angle/pi) $* 180$. With the missing semi-colon, Matlab will do the computations and print the result to the screen. And yes, the famous pi $(\pi)$ is a variable that is known to Matlab.

\subsection{A Matlab Program with Vectorization and Plotting}

We return to the problem where a ball is thrown up in the air and we have a formula for the vertical position $y$ of the ball. Say we are interested in $y$ at every millisecond for the first second of the flight. This requires repeating the calculation of $y=v_{0} t-0.5 g t^{2}$ one thousand times.

We will also draw a graph of $y$ versus $t$ for $t \in[0,1]$. Drawing such graphs on a computer essentially means drawing straight lines between points on the curve, so we need many points to make the visual impression of a smooth curve. With one thousand points, as we aim to compute here, the curve looks indeed very smooth.

In Matlab, the calculations and the visualization of the curve may be done with the program ball_plot.m, reading

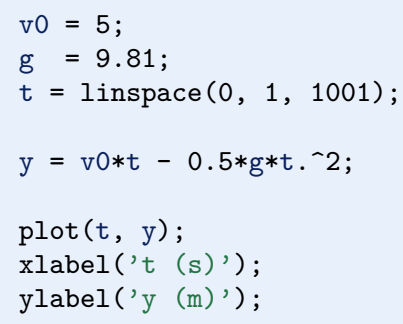

This program produces a plot of the vertical position with time, as seen in Fig. 1.1. As you notice, the code lines from the ball .m program in Sect. 1.2 have not changed much, but the height is now computed and plotted for a thousand points in time!

Let us take a look at the differences between the new program and our previous program.

The function linspace takes 3 parameters, and is generally called as 


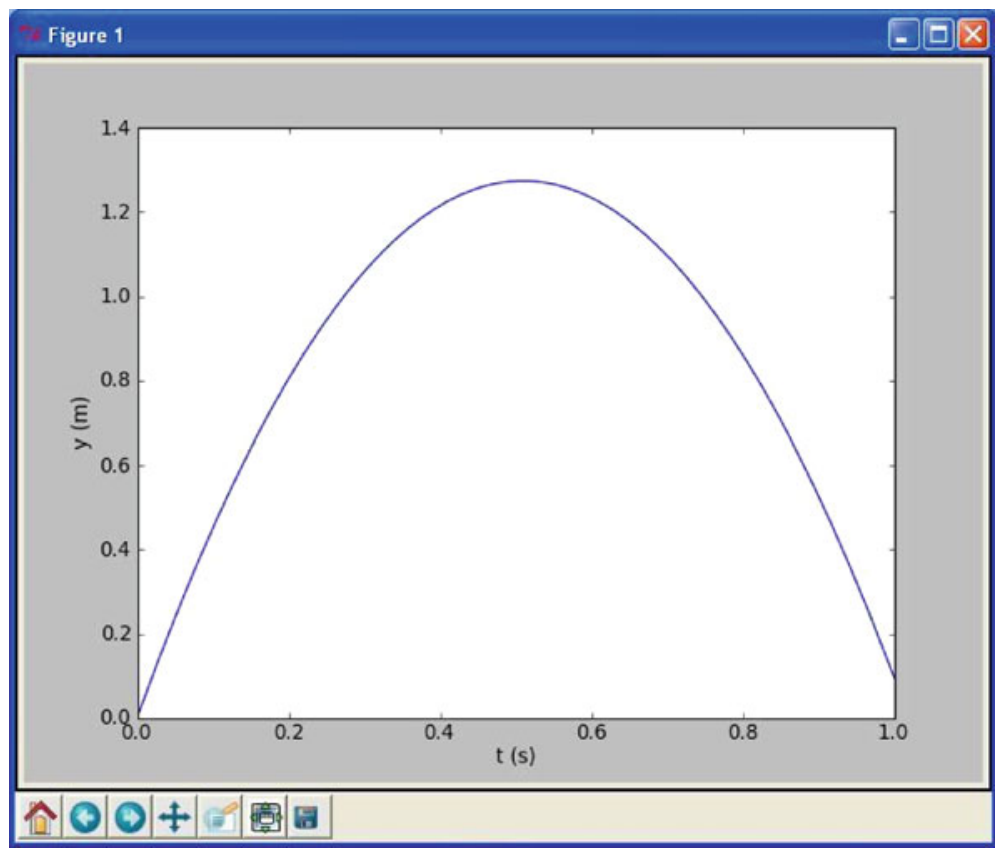

Fig. 1.1 Plot generated by the script ball_plot.m, showing the vertical position of the ball at a thousand points in time

This is our first example of a Matlab function that takes multiple arguments. The linspace function generates $\mathrm{n}$ equally spaced coordinates, starting with start and ending with stop. The expression linspace $(0,1,1001)$ creates 1001 coordinates between 0 and 1 (including both 0 and 1). The mathematically inclined reader will notice that 1001 coordinates correspond to 1000 equal-sized intervals in $[0,1]$ and that the coordinates are then given by $t_{i}=i / 1000(i=0,1, \ldots, 1000)$.

The value returned from linspace (being stored in $t$ ) is an array, i.e., a collection of numbers. When we start computing with this collection of numbers in the arithmetic expression $\mathrm{v} 0 * \mathrm{t}-0.5 * \mathrm{~g} * \mathrm{t} .{ }^{\wedge} 2$, the expression is calculated for every number in $\mathrm{t}$ (i.e., every $t_{i}$ for $i=0,1, \ldots, 1000$ ), yielding a similar collection of 1001 numbers in the result $\mathrm{y}$. That is, $\mathrm{y}$ is also an array.

Note the dot that has been inserted in $0.5 * \mathrm{~g} * \mathrm{t} .{ }^{\wedge} 2$, i.e. just before the operator ^ . This is required to make Matlab do - to each number in $t$. The same thing applies to other operators, as shown in several examples later.

This technique of computing all numbers "in one chunk" is referred to as vectorization. When it can be used, it is very handy, since both the amount of code and computation time is reduced compared to writing a corresponding for or while loop (Chap. 2) for doing the same thing.

The plotting commands are simple:

1. $p l o t(t, y)$ means plotting all the $y$ coordinates versus all the $t$ coordinates

2. xlabel ('t (s)') places the text $t$ ( $s$ ) on the $x$ axis

3. ylabel ('y (m)') places the text y (m) on the $y$ axis 
At this stage, you are strongly encouraged to do Exercise 1.4. It builds on the example above, but is much simpler both with respect to the mathematics and the amount of numbers involved.

\subsection{More Basic Concepts}

So far we have seen a few basic examples on how to apply Matlab programming to solve mathematical problems. Before we can go on with other and more realistic examples, we need to briefly treat some topics that will be frequently required in later chapters. These topics include computer science concepts like variables, objects, error messages, and warnings; more numerical concepts like rounding errors, arithmetic operator precedence, and integer division; in addition to more Matlab functionality when working with arrays, plotting, and printing.

\subsubsection{Using Matlab Interactively}

You may also use Matlab interactively (i.e. without writing a program). For example, you may do calculations like the following directly at the command prompt >> in the Command window (a prompt means a "ready sign", i.e. the program allows you to enter a command, and different programs often have different looking prompts).

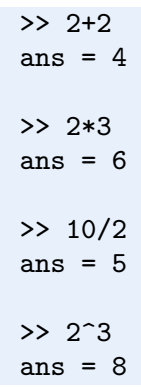

You may also define variables and use formulas interactively as

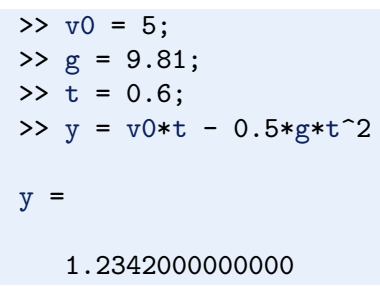

Sometimes you would like to repeat a command you have given earlier, or perhaps give a command that is almost the same as an earlier one. Then you can use the "up-arrow" key. Pressing this one time gives you the previous command, pressing two times gives you the command before that, and so on. If you go too far, you may 
go back with the "down-arrow" key. When you have the command at the prompt, it may be edited before pressing enter (which lets Matlab read it).

\subsubsection{Arithmetics, Parentheses and Rounding Errors}

When the arithmetic operators $+,-, *, /$ and - appear in an expression, Matlab gives them a certain precedence. Matlab interprets the expression from left to right, taking one term (part of expression between two successive + or - ) at a time. Within each term, - is done before $*$ and /. Consider the expression $\mathrm{x}=1 * 5 \sim 2+10 * 3-1.0 / 4$. There are three terms here and interpreting this, Matlab starts from the left. In the first term, $1 * 5^{\wedge} 2$, it first does $5^{\wedge} 2$ which equals 25 . This is then multiplied by 1 to give 25 again. The second term is $10 * 3$, i.e., 30 . So the first two terms add up to 55 . The last term gives 0.25 , so the final result is 54.75 which becomes the value of $\mathrm{x}$.

Note that parentheses are often very important to group parts of expressions together in the intended way. Let us say $\mathrm{x}=4$ and that you want to divide 1.0 by $\mathrm{x}+1$. We know the answer is 0.2 , but the way we present the task to Matlab is critical, as shown by the following example.

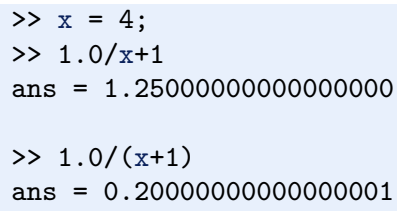

In the first try, we see that 1.0 is divided by $x$ (i.e., 4), giving 0.25 , which is then added to 1. Matlab did not understand that our complete denominator was $x+1$. In our second try, we used parentheses to "group" the denominator, and we got what we wanted. That is, almost what we wanted! Since most numbers can be represented only approximately on the computer, this gives rise to what is called rounding errors. We should have got 0.2 as our answer, but the inexact number representation gave a small error. Usually, such errors are so small compared to the other numbers of the calculation, that we do not need to bother with them. Still, keep it in mind, since you will encounter this issue from time to time. More details regarding number representations on a computer is given in Sect. 3.4.3.

\subsubsection{Variables}

Variables in Matlab will be of a certain type. Real numbers are in computer language referred to as floating point numbers, being the default (i.e. what Matlab uses if nothing is specified) data type in Matlab. These are of two kinds, single and double, corresponding to single and double precision, respectively. It is the "double" that is the default type. With double precision, twice as many bits (64) are used for storage as with single precision. Writing $\mathrm{x}=2$ in some Matlab program, by default makes $x$ a double, i.e. a float variable. 
Whole numbers may be stored more memory efficiently as integers, however, and there are several ways of doing this. For example, writing $\mathrm{x}=\operatorname{int8}(2)$, or $\mathrm{x}=$ int16(2), will store the integer 2 in the variable $\mathrm{x}$ by use of 8 or 16 bits, respectively.

Another common type of variable is a string, which we get by writing, e.g., $\mathrm{x}=$ 'This is a string'. The variable $\mathrm{x}$ then becomes a string variable containing the text between single quotes (the string actually becomes an array of characters, see "Arrays" below). Several other standard data types also exist in Matlab.

You may also convert between variable types in different ways. For example, after first writing $\mathrm{x}=2$ (which causes $\mathrm{x}$ to become a double), you may write $\mathrm{y}$ $=$ single $(\mathrm{x})$ to make $\mathrm{y}$ contain the number 2 with single precision only. Type conversion may also occur automatically, e.g. when calling a ready-made Matlab function that requires input data to be of a certain type. When combining variables of different types, the result will have a type according to given rules. For example, multiplying a single and a double, gives a single variable.

Names of variables should be chosen so that they are descriptive. When computing a mathematical quantity that has some standard symbol, e.g. $\alpha$, this should be reflected in the name by letting the word alpha be part of the name for the corresponding variable in the program. If you, e.g., have a variable for counting the number of sheep, then one appropriate name could be no_of_sheep. Such naming makes it much easier for a human to understand the written code. Variable names may also contain any digit from 0 to 9 , or underscores, but can not start with a digit. Letters may be lower or upper case, which to Matlab is different. Note that certain names in Matlab are reserved, meaning that you can not use these as names for variables. Some examples are for, while, if, else, end, global and function. If you accidentally use a reserved word as a variable name you get an error message.

We have seen that, e.g., $\mathrm{x}=2$ will assign the value 2 to the variable $\mathrm{x}$. But how do we write it if we want to increase $x$ by 4 ? We may then write an assignment like $\mathrm{x}=\mathrm{x}+4$. Now Matlab interprets this as: "take whatever value that is in $\mathrm{x}$, add 4, and let the result become the new value of ' $\mathrm{x}$ "'. In other words, the old value of $\mathrm{x}$ is used on the right hand side of $=$, no matter how messy the expression might be, and the result becomes the new value of $x$. In a similar way, $x=x-4$ reduces the value of $\mathrm{x}$ by $4, \mathrm{x}=\mathrm{x} * 4$ multiplies $\mathrm{x}$ by 4 , and $\mathrm{x}=\mathrm{x} / 4$ divides $\mathrm{x}$ by 4 , updating the value of $\mathrm{x}$ accordingly.

\subsubsection{Formatting Text and Numbers}

Results from scientific computations are often to be reported as a mixture of text and numbers. Usually, we want to control how numbers are formatted. For example, we may want to write $1 / 3$ as 0.33 or $3.3333 e-01\left(3.3333 \cdot 10^{-1}\right)$. The fprintf command is the key tool to write out text and numbers with full control of the formatting. The first argument to fprintf is a string with a particular syntax to specify the formatting, the so-called printf syntax. (The peculiar name stems from the printf function in the programming language $\mathrm{C}$ where the syntax was first introduced.)

Suppose we have a real number 12.89643, an integer 42, and a text 'some message' that we want to write out in the following two alternative ways: 
real $=12.896$, integer $=42$, string=some message

real=1.290e+01, integer $=42$, string=some message

The real number is first written in decimal notation with three decimals, as 12.896, but afterwards in scientific notation as $1.290 \mathrm{e}+01$. The integer is first written as compactly as possible, while on the second line, 42 is formatted in a text field of width equal to five characters.

The following program, formatted_print.m, applies the printf syntax to control the formatting displayed above:

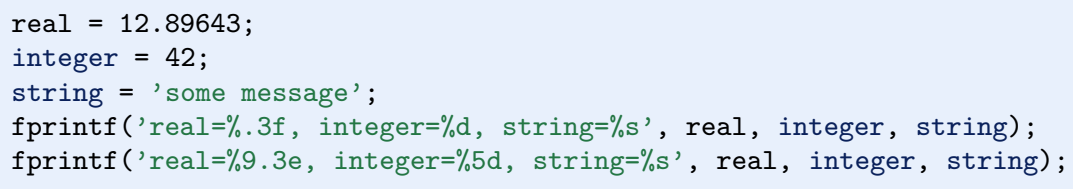

The output of fprintf is a string, specified in terms of text and a set of variables to be inserted in the text. Variables are inserted in the text at places indicated by $\%$. After $\%$ comes a specification of the formatting, e.g., $\%$ f (real number), $\% \mathrm{~d}$ (integer), or $\%$ s (string). The format $\% 9.3$ means a real number in decimal notation, with 3 decimals, written in a field of width equal to 9 characters. The variant $\% .3 f$ means that the number is written as compactly as possible, in decimal notation, with three decimals. Switching $f$ with e or E results in the scientific notation, here $1.290 e+01$ or $1.290 \mathrm{E}+01$. Writing $\% 5 \mathrm{~d}$ means that an integer is to be written in a field of width equal to 5 characters. Real numbers can also be specified with $\% g$, which is used to automatically choose between decimal or scientific notation, from what gives the most compact output (typically, scientific notation is appropriate for very small and very large numbers and decimal notation for the intermediate range).

A typical example of when printf formatting is required, arises when nicely aligned columns of numbers are to be printed. Suppose we want to print a column of $t$ values together with associated function values $g(t)=t \sin (t)$ in a second column. The simplest approach would be

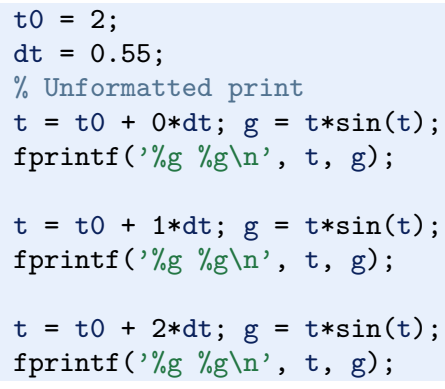

with output 
(Repeating the same set of statements multiple times, as done above, is not good programming practice - one should use a for loop, as explained later in Sect. 2.3.) Observe that the numbers in the columns are not nicely aligned. Using the printf syntax $\% 6.2 f \% 8.3 f, \%$ ( $t, g)$ for $t$ and $g$, we can control the width of each column and also the number of decimals, such that the numbers in a column are aligned under each other and written with the same precision. The output then becomes

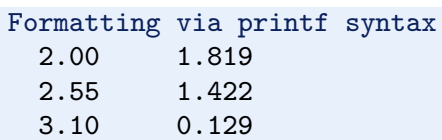

We shall frequently use the printf syntax throughout the book so there will be plenty of further examples.

\subsubsection{Arrays}

In the program ball_plot.m from Sect. 1.4 we saw how 1001 height computations were executed and stored in the variable $y$, and then displayed in a plot showing $y$ versus $t$, i.e., height versus time. The collection of numbers in y (or $t$, respectively) was stored in what is called an array, a construction also found in most other programming languages. Such arrays are created and treated according to certain rules, and as a programmer, you may direct Matlab to compute and handle arrays as a whole, or as individual array elements. Let us briefly look at a smaller such collection of numbers.

Assume that the heights of four family members have been collected. These heights may be generated and stored in an array, e.g., named h, by writing

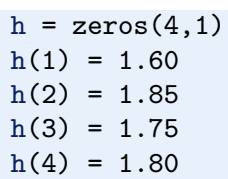

where the array elements appear as $\mathrm{h}(1), \mathrm{h}(2)$, etc. Generally, when we read or talk about the array elements of some array a, we refer to them by reading or saying "a of one" (i.e. a (1)), "a of two" (i.e. a (2)), and so on. The very first line in the example above, i.e.

$$
\mathrm{h}=\operatorname{zeros}(4,1)
$$

instructs Matlab to reserve, or allocate, space in memory for an array $\mathrm{h}$ with four elements and initial values set to 0 . (Note that zeros $(4,1)$ gives a column array, while zeros $(1,4)$ gives a line array. Try it at the command prompt to see the difference. Sometimes this distinction is important, e.g. when doing matrix - vector calculations.) The next four lines command Matlab to overwrite the zeros with the desired numbers (measured heights), one number for each element. Elements are, by rule, indexed (numbers within parentheses) from 1 to the last element, in this 
case 4. We say that Matlab has one-based indexing. This differs from zero-based indexing (e.g., found in Python) where the array index starts with 0 .

As illustrated in the code, you may refer to the array as a whole by the name $\mathrm{h}$, but also to each individual element by use of the index. The array elements may enter in computations as individual variables, e.g., writing $z=h(1)+h(2)+$ $h(3)+h(4)$ will compute the sum of all the elements in $h$, while the result is assigned to the variable $z$. Note that this way of creating an array is a bit different from the one with linspace, where the filling in of numbers occurred automatically "behind the scene".

By use of a colon, you may pick out a slice of an array. For example, to create a new array from the two elements $h(1)$ and $h(2)$, we could write slice_h $=h(1: 2)$. For the generated slice_h array, indices are as usual, i.e., 1 and 2 in this case. The very last entry in an array may be addressed as, e.g., $h$ (length $(h)$ ), where the ready made function length gives the number of elements in the array.

\subsubsection{Plotting}

Sometimes you would like to have two or more curves or graphs in the same plot. Assume we have $\mathrm{h}$ as above, and also an array $\mathrm{H}$ with the heights $0.50 \mathrm{~m}, 0.70 \mathrm{~m}$, $1.90 \mathrm{~m}$, and $1.75 \mathrm{~m}$ from a family next door. This may be done with the program plot_heights.m given as

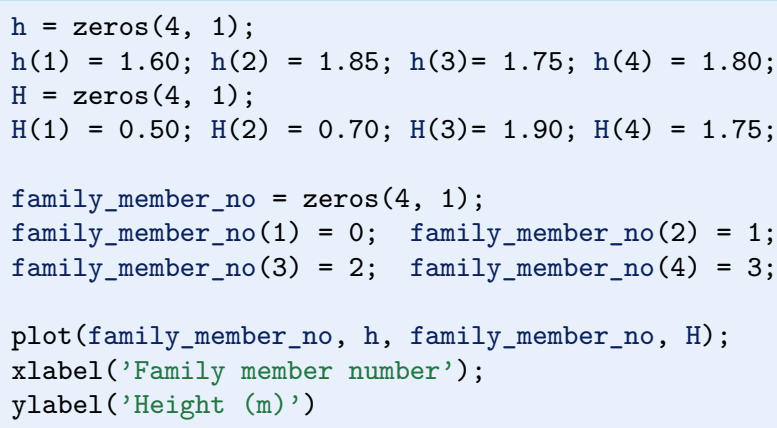

Running the program gives the plot shown in Fig. 1.2.

Alternatively, the two curves could have been plotted in the same plot by use of two plot commands, which gives more freedom as to how the curves appear. To do this, you could plot the first curve by

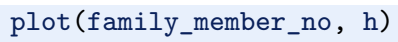

Then you could (in principle) do a lot of other things in your code, before you plot the second curve by 


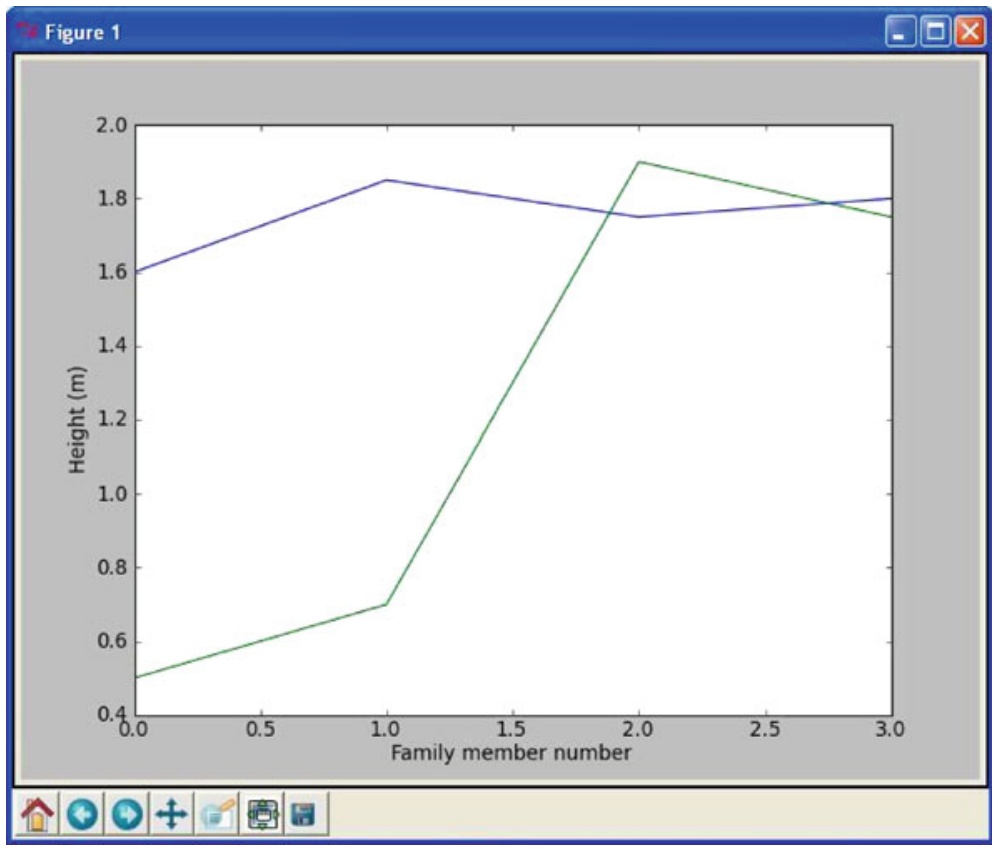

Fig. 1.2 Generated plot for the heights of family members from two families

Notice the use of hold here. hold ('on') tells Matlab to plot also the following curve(s) in the same window. Matlab does so until it reads hold('off'). If you do not use the hold ('on') or hold ('off') command, the second plot command will overwrite the first one, i.e., you get only the second curve.

In case you would like the two curves plotted in two separate plots, you can do this by plotting the first curve straightforwardly with

plot (family_member_no, h)

then do other things in your code, before you do

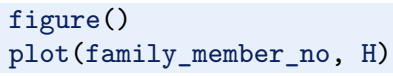

Note how the graphs are made continuous by Matlab, drawing straight lines between the four data points of each family. This is the standard way of doing it and was also done when plotting our 1001 height computations with ball_plot.m in Sect. 1.4. However, since there were so many data points then, the curve looked nice and smooth. If preferred, one may also plot only the data points. For example, writing

$\operatorname{plot}\left(\mathrm{h},{ }^{\prime} *^{\prime}\right)$ 
will mark only the data points with the star symbol. Other symbols like circles etc. may be used as well.

There are many possibilities in Matlab for adding information to a plot or for changing its appearance. For example, you may add a legend by the instruction

legend('This is some legend')

or you may add a title by

title('This is some title')

The command

$\operatorname{axis}([\mathrm{xmin}$ xmax ymin $y \max ])$

will define the plotting range for the $x$ axis to stretch from xmin to xmax and, similarly, the plotting range for the $y$ axis from ymin to ymax. Saving the figure to file is achieved by the command

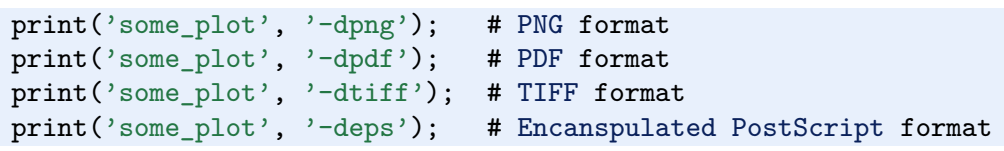

For the reader who is into linear algebra, it may be useful to know that standard matrix/vector operations are straightforward with arrays, e.g., matrix-vector multiplication. For example, assume you would like to calculate the vector $\mathbf{y}$ (note that boldface is used for vectors and matrices) as $\mathbf{y}=\mathbf{A x}$, where $\mathbf{A}$ is a $2 \times 2$ matrix and $\mathbf{x}$ is a vector. We may do this as illustrated by the program matrix_vector_product.m reading

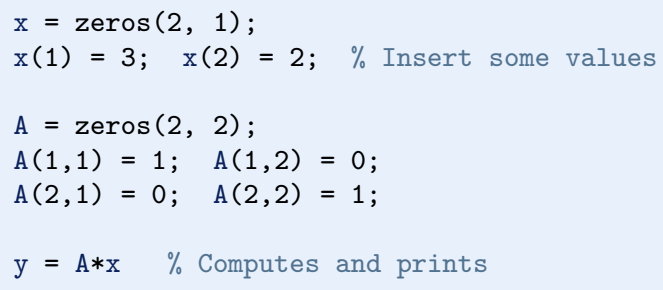

Here, $\mathrm{x}$ is first established as a column array with the zeros function. Then the test values are plugged in ( 3 and 2). The matrix A is first created as a two dimensional array with $A=\operatorname{zeros}(2,2)$ before filling in values. Finally, the multiplication is performed as $\mathrm{y}=\mathrm{A} * \mathrm{x}$. Running the program gives the following output on the screen:

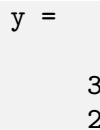




\subsubsection{Error Messages and Warnings}

All programmers experience error messages, and usually to a large extent during the early learning process. Sometimes error messages are understandable, sometimes they are not. Anyway, it is important to get used to them. One idea is to start with a program that initially is working, and then deliberately introduce errors in it, one by one. (But remember to take a copy of the original working code!) For each error, you try to run the program to see what Matlab's response is. Then you know what the problem is and understand what the error message is about. This will greatly help you when you get a similar error message or warning later.

Very often, you will experience that there are errors in the program you have written. This is normal, but frustrating in the beginning. You then have to find the problem, try to fix it, and then run the program again. Typically, you fix one error just to experience that another error is waiting around the corner. However, after some time you start to avoid the most common beginner's errors, and things run more smoothly. The process of finding and fixing errors, called debugging, is very important to learn. There are different ways of doing it too.

A special program (debugger) may be used to help you check (and do) different things in the program you need to fix. A simpler procedure, that often brings you a long way, is to print information to the screen from different places in the program. First of all, this is something you should do (several times) during program development anyway, so that things get checked as you go along. However, if the final program still ends up with error messages, you may save a copy of it, and do some testing on the copy. Useful testing may then be to remove, e.g., the latter half of the program (by inserting comment signs \%), and insert print commands at clever places to see what is the case. When the first half looks ok, insert parts of what was removed and repeat the process with the new code. Using simple numbers and doing this in parallel with hand calculations on a piece of paper (for comparison) is often a very good idea.

Matlab also offers means to detect and handle errors by the program itself! The programmer must then foresee (when writing the code) that there is a potential for error at some particular point. If, for example, some user of the program is asked (by the running program) to provide a number, and intends to give the number 5, but instead writes the word five, the program could run into trouble. A try-catch construction may be used by the programmer to check for such errors and act appropriately (see Sect. 6.2 for an example), avoiding a program crash. This procedure of trying an action and then recovering from trouble, if necessary, is referred to as exception handling and is the modern way of dealing with errors in a program.

When a program finally runs without error messages, it might be tempting to think that Ah ..., I am finished!. But no! Then comes program testing, you need to verify that the program does the computations as planned. This is almost an art and may take more time than to develop the program, but the program is useless unless you have much evidence showing that the computations are correct. Also, having a set of (automatic) tests saves huge amounts of time when you further develop the program. 


\section{Verification versus validation}

Verification is important, but validation is equally important. It is great if your program can do the calculations according to the plan, but is it the right plan? Put otherwise, you need to check that the computations run correctly according to the formula you have chosen/derived. This is verification: doing the things right. Thereafter, you must also check whether the formula you have chosen/derived is the right formula for the case you are investigating. This is validation: doing the right things. In the present book, it is beyond scope to question how well the mathematical models describe a given phenomenon in nature or engineering, as the answer usually involves extensive knowledge of the application area. We will therefore limit our testing to the verification part.

\subsubsection{Input Data}

Computer programs need a set of input data and the purpose is to use these data to compute output data, i.e., results. In the previous program we have specified input data in terms of variables. However, one often wants to get the input through some dialog with the user. Here is one example where the program asks a question, and the user provides an answer by typing on the keyboard:

age = input ('What is your age? ')

fprintf('Ok, so you are half way to $\%$ d, wow! $\backslash n^{\prime}$, age*2)

So, after having interpreted and run the first line, Matlab has established the variable age and assigned your input to it. The second line combines the calculation of twice the age with a message printed on the screen. Try these two lines in a little test program to see for yourself how it works.

There are other ways of providing input to a program as well, e.g., via a graphical interface (as many readers will be used to) or at the command line (i.e., as parameters succeeding, on the same line, the command that starts the program). Reading data from a file is yet another way. Logically, what the program produces when run, e.g. a plot or printout to the screen or a file, is referred to as program output.

\subsubsection{Symbolic Computations}

Even though the main focus in this book is programming of numerical methods, there are occasions where symbolic (also called exact or analytical) operations are useful. Doing symbolic computations means, as the name suggests, that we do computations with the symbols themselves rather than with the numerical values they could represent. Let us illustrate the difference between symbolic and numerical computations with a little example. A numerical computation could be

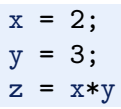


which will make the number 6 appear on the screen. A symbolic counterpart of this code could be

syms $\mathrm{x} y$

$\mathrm{z}=\mathrm{x} * \mathrm{y}$

which causes the symbolic result $\mathrm{x} * \mathrm{y}$ to appear on the screen. Note that no numerical value was assigned to any of the variables in the symbolic computation. Only the symbols were used, as when you do symbolic mathematics by hand on a piece of paper.

Symbolic computations in Matlab make use of the Symbolic Toolbox (but support for symbolic computations in Octave is weak). Each symbol is represented by a standard variable, but the name of the symbol must be declared with syms name for a single symbol, or syms name1 name2 ... for multiple symbols. The following script example_symbolic.mis a quick demonstration of some of the basic symbolic operations that are supported in Matlab.

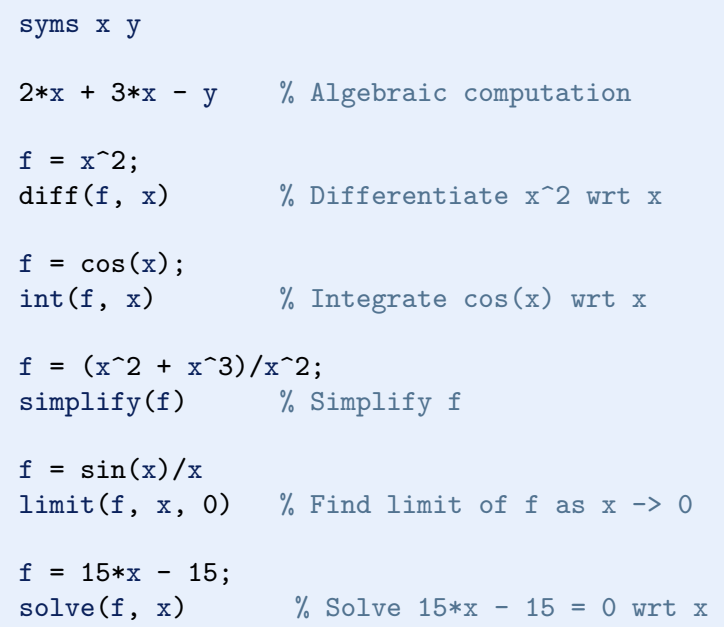

Other symbolic calculations like Taylor series expansion, linear algebra (with matrix and vector operations), and (some) differential equation solving are also possible.

Symbolic computations are also readily accessible through the (partly) free online tool WolframAlpha ${ }^{5}$, which applies the very advanced Mathematica ${ }^{6}$ package as symbolic engine. The disadvantage with WolframAlpha compared to the Symbolic Toolbox is that the results cannot automatically be imported into your code and used for further analysis. On the other hand, WolframAlpha has the advantage that it displays many additional mathematical results related to the given problem. For example, if we type $2 \mathrm{x}+3 \mathrm{x}-\mathrm{y}$ in WolframAlpha, it not only simplifies the expression to $5 \mathrm{x}-\mathrm{y}$, but it also makes plots of the function $f(x, y)=5 x-y$, solves the equation $5 x-y=0$, and calculates the integral $\iint(5 x+y) d x d y$.

\footnotetext{
${ }^{5}$ http://www.wolframalpha.com

${ }^{6} \mathrm{http} / / /$ en.wikipedia.org/wiki/Mathematica
} 
The commercial Pro version can also show a step-by-step of the analytical computations in the problem. You are strongly encouraged to try out these commands in WolframAlpha:

- $\operatorname{diff}\left(\mathrm{x}^{\wedge} 2, x\right)$ or $\operatorname{diff}(x * * 2, x)$

- integrate $(\cos (x), x)$

- $\operatorname{simplify}((\mathrm{x} * * 2+\mathrm{x} * * 3) / \mathrm{x} * * 2)$

- $\operatorname{limit}(\sin (x) / x, x, 0)$

- $\operatorname{solve}(5 * x-15, x)$

WolframAlpha is very flexible with respect to syntax.

Another impressive tool for symbolic computations is $\mathrm{Sage}^{7}$, which is a very comprehensive package with the aim of "creating a viable free open source alternative to Magma, Maple, Mathematica and Matlab". Sage is implemented in Python. Projects with extensive symbolic computations will certainly benefit from exploring Sage.

\subsubsection{Concluding Remarks}

\section{Programming demands you to be accurate!}

In this chapter, you have seen some examples of how simple things may be done in Matlab. Hopefully, you have tried to do the examples on your own. If you have, most certainly you have discovered that what you write in the code has to be very accurate. For example, with our previous example of four heights collected in an array h, writing $h[1]$ instead of $h(1)$ gives an error, even if you and I know perfectly well what you mean! Remember that it is not a human that runs your code, it is a machine. Therefore, even if the meaning of your code looks fine to a human eye, it still has to comply in detail to the rules of the programming language. If not, you get warnings and error messages. This also goes for lower and upper case letters. Pay attention to such details also when they are given in later chapters.

\section{Remember to insert comments to explain your code}

When you write a computer program, you have two very different kinds of readers. One is Matlab, which will interpret and run your program according to the rules. The other is some human, for example, yourself or a peer. It is very important to organize and comment the code so that you can go back to your own code after, e.g., a year and still understand what clever constructions you put in there. This is relevant when you need to change or extend your code (which usually happens often in reality). Organized coding and good commenting is even more critical if other people are supposed to understand code that you have written.

One important contribution to writing readable code, is to indent parts of the code that naturally belong together. You will see this used systematically from Chap. 2 and on. It is a highly recommendable habit to develop for a programmer.

\footnotetext{
${ }^{7}$ http://sagemath.org/
} 


\section{Fast code versus readable and correct code}

Numerical computing has a strong tradition in paying much attention to creating fast code. Real industrial applications of numerical computing often involves simulations that run for hours, days, and even weeks. Fast code is tremendously important in those cases. The problem with a strong focus on fast code, unfortunately, is sometimes that clear and easily understandable constructions are replaced by clever and less readable, but faster code. However, for beginners it is most important to learn to write readable and correct code. We will make some comments on constructions that are fast or slow, but the main focus of this book is to teach how to write correct programs, not the fastest possible programs.

\section{Matlab requires a license}

Matlab has a student licence version that is cheap as long as you are a student. Note, however, that the student version is stripped of much functionality. A commercial license is required to use Matlab in industry.

Matlab has a whole range of toolboxes with ready-made code dedicated to particular fields in science and engineering. We encountered one of these above, the Symbolic Toolbox. Generally, the more toolboxes you want to include in your license, the more expensive it gets.

\section{Tip: how to deal with long lines}

If a statement in a program gets too long, it may be continued on the next line by inserting three dots in succession immediately after the last character of the line that is split (no spaces between!).

The present introductory book just provides a tiny bit of all the functionality that Matlab has to offer. An important source of information is the homepage "of Matlab": http://www.mathworks.com. In addition, there are lots of excellent books (for references, see Preface).

\subsection{Exercises}

\section{Exercise 1.1: Error messages}

Save a copy of the program ball.m and confirm that the copy runs as the original. You are now supposed to introduce errors in the code, one by one. For each error introduced, save and run the program, and comment how well Matlab's response corresponds to the actual error. When you are finished with one error, re-set the program to correct behavior (and check that it works!) before moving on to the next error.

a) Insert the word hello on the empty line above the assignment to v0.

b) Remove the $\%$ sign in front of the comment initial velocity.

c) Remove the = sign in the assignment to v0.

d) Change the symbol - into **.

e) Change the calculation of y to $y=v 0 * t$.

f) Write $x$ on the line just above where $y$ is calculated. 
g) Change the statement $\mathrm{y}=\mathrm{v} 0 * \mathrm{t}-0.5 * \mathrm{~g} * \mathrm{t} \sim 2$ into $\mathrm{y}=\mathrm{v} 0 * \mathrm{t}-0.5 * \mathrm{~g} * \mathrm{t} \sim 2$; That is, insert a semicolon at the end.

Filename: testing_ball.m.

\section{Exercise 1.2: Volume of a cube}

Write a program that computes the volume $V$ of a cube with sides of length $L=$ $4 \mathrm{~cm}$ and prints the result to the screen. Both $V$ and $L$ should be defined as separate variables in the program. Run the program and confirm that the correct result is printed.

Hint See ball.m in the text.

Filename: cube_volume.m.

\section{Exercise 1.3: Area and circumference of a circle}

Write a program that computes both the circumference $C$ and the area $A$ of a circle with radius $r=2 \mathrm{~cm}$. Let the results be printed to the screen on a single line with an appropriate text. The variables $C, A$ and $r$ should all be defined as separate variables in the program. Run the program and confirm that the correct results are printed.

Filename: circumference_and_area.m.

\section{Exercise 1.4: Volumes of three cubes}

We are interested in the volume $V$ of a cube with length $L: V=L^{3}$, computed for three different values of $L$.

a) Use the linspace function to compute three values of $L$, equally spaced on the interval $[1,3]$.

b) Carry out by hand the computation $V=L^{3}$ if $L$ is an array with three elements. That is, compute $V$ for each value of $L$.

c) In a program, write out the result $\mathrm{V}$ of $\mathrm{V}=\mathrm{L} . \wedge-3$ when $\mathrm{L}$ is an array with three elements as computed by linspace in a). Compare the resulting volumes with your hand calculations.

d) Make a plot of V versus L.

Filename: volume3cubes.m.

\section{Exercise 1.5: Average of integers}

Write a program that stores the sum $1+2+3+4+5$ in one variable and then creates another variable with the average of these five numbers. Print the average to the screen and check that the result is correct.

Filename: average_int.m.

\section{Exercise 1.6: Interactive computing of volume and area}

a) Compute the volume in Exercise 1.2 by using Matlab interactively, i.e., do the computations at the command prompt (in a Matlab shell as we also say). Compare with what you got previously from the written program.

b) Do the same also for Exercise 1.3. 


\section{Exercise 1.7: Update variable at command prompt}

Invoke Matlab interactively and perform the following steps.

1. Initialize a variable $\mathrm{x}$ to 2 .

2. Add 3 to $x$. Print out the result.

3. Print out the result of $x+1 * 2$ and $(x+1) * 2$. (Observe how parentheses make a difference).

4. What variable type is $\mathrm{x}$ ?

\section{Exercise 1.8: Formatted print to screen}

Write a program that defines two variables as $x=p i$ and $y=2$. Then let the program compute the product $z$ of these two variables and print the result to the screen as

Multiplying 3.14159 and 2 gives 6.283

Filename: formatted_print.m.

\section{Exercise 1.9: Matlab documentation and random numbers}

Write a program that prints four random numbers to the screen. The numbers should be drawn from a uniform distribution over the interval $[0,10)$ (0 inclusive, 10 exclusive). Find the information needed for the task, see for example http://www. mathworks.com.

Hint Matlab has a built-in function rand for drawing random numbers. Try $\gg$ help rand at the command prompt.

Filename: drawing_random_numbers.m.

Open Access This chapter is distributed under the terms of the Creative Commons AttributionNonCommercial 4.0 International License (http://creativecommons.org/licenses/by-nc/4.0/), which permits any noncommercial use, duplication, adaptation, distribution and reproduction in any medium or format, as long as you give appropriate credit to the original author(s) and the source, a link is provided to the Creative Commons license and any changes made are indicated.

The images or other third party material in this chapter are included in the work's Creative Commons license, unless indicated otherwise in the credit line; if such material is not included in the work's Creative Commons license and the respective action is not permitted by statutory regulation, users will need to obtain permission from the license holder to duplicate, adapt or reproduce the material. 


\section{Basic Constructions}

Python 2.7

\# Square elements of a list

$x=[0,0.2,0.4,1,2,4]$

$y=[]$

for $1, x$ in enumerate $(x)$ :

$y$. append $\left(x_{-} * 2\right)$

print $y$

Edit code

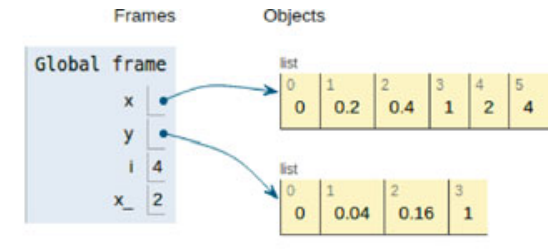

\subsection{If Tests}

Very often in life, and in computer programs, the next action depends on the outcome of a question starting with "if". This gives the possibility to branch into different types of action depending on some criterion. Let us as usual focus on a specific example, which is the core of so-called random walk algorithms used in a wide range of branches in science and engineering, including materials manufacturing and brain research. The action is to move randomly to the north $(\mathrm{N})$, east $(\mathrm{E})$, south (S), or west (W) with the same probability. How can we implement such an action in life and in a computer program?

We need to randomly draw one out of four numbers to select the direction in which to move. A deck of cards can be used in practice for this purpose. Let the four suits correspond to the four directions: clubs to $\mathrm{N}$, diamonds to $\mathrm{E}$, hearts to $\mathrm{S}$, and spades to $\mathrm{W}$, for instance. We draw a card, perform the corresponding move, and repeat the process a large number of times. The resulting path is a typical realization of the path of a diffusing molecule.

In a computer program, we need to draw a random number, and depending on the number, update the coordinates of the point to be moved. There are many ways to draw random numbers and translate them into (e.g.) four random directions, but the technical details usually depend on the programming language. Our technique here is universal: we draw a random number in the interval $[0,1)$ and let $[0,0.25)$ correspond to $\mathrm{N},[0.25,0.5)$ to $\mathrm{E},[0.5,0.75)$ to $\mathrm{S}$, and $[0.75,1)$ to W. Let $\mathrm{x}$ and $\mathrm{y}$ hold the coordinates of a point and let $d$ be the length of the move. A pseudo code (i.e., not "real" code, just a "sketch of the logic") then goes like 


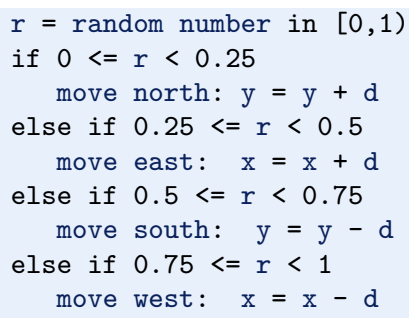

Note the need for first asking about the value of $r$ and then performing an action. If the answer to the "if" question is positive (true), we are done and can skip the next else if questions. If the answer is negative (false), we proceed with the next question. The last test if $0.75 \leq r<1$ could also read just else, since we here cover all the remaining possible $r$ values.

The exact code in Matlab reads

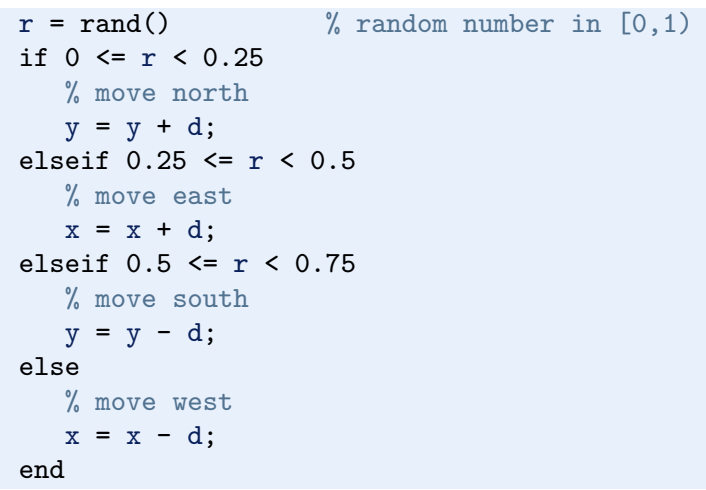

We use else in the last test to cover the different types of syntax that is allowed. Matlab recognizes the reserved words if, elseif, and else and expects the code to be compatible with the rules of if tests:

- The test reads if condition, elseif condition, or else, where condition is a boolean expression that evaluates to true (1) or false (0).

- If condition is true, the following statements up to the next elseif, else, or end are executed, and the remaining elseif or else branches are skipped.

- If condition is false, the program flow jumps to the next elseif or else branch.

The blocks after if, elseif, or else may contain new if tests, if desired.

Working with if tests requires mastering boolean expressions. Here are some basic boolean expressions involving the logical operators $==,=,<,<=,>$, and $>=$. Given the assignment to temp, you should go through each boolean expression below and determine if it is true or false. 


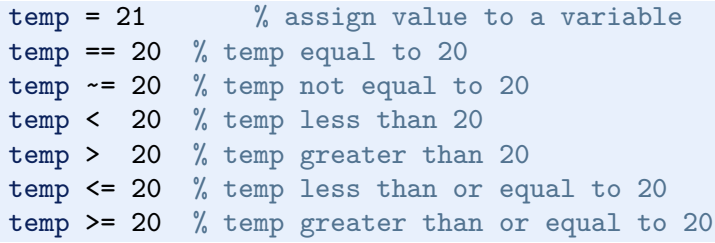

\subsection{Functions}

Functions are widely used in programming and is a concept that needs to be mastered. In the simplest case, a function in a program is much like a mathematical function: some input number $x$ is transformed to some output number. One example is the $\tanh ^{-1}(x)$ function, called atan in computer code: it takes one real number as input and returns another number. Functions in Matlab are more general and can take a series of variables as input and return one or more variables, or simply nothing. The purpose of functions is two-fold:

1. to group statements into separate units of code lines that naturally belong together (a strategy which may dramatically ease the problem solving process), and/or

2. to parameterize a set of statements such that they can be written only once and easily be re-executed with variations.

Examples will be given to illustrate how functions can be written in various contexts.

If we modify the program ball .m from Sect. 1.2 slightly, and include a function, we could let this be a new program ball_function.mas

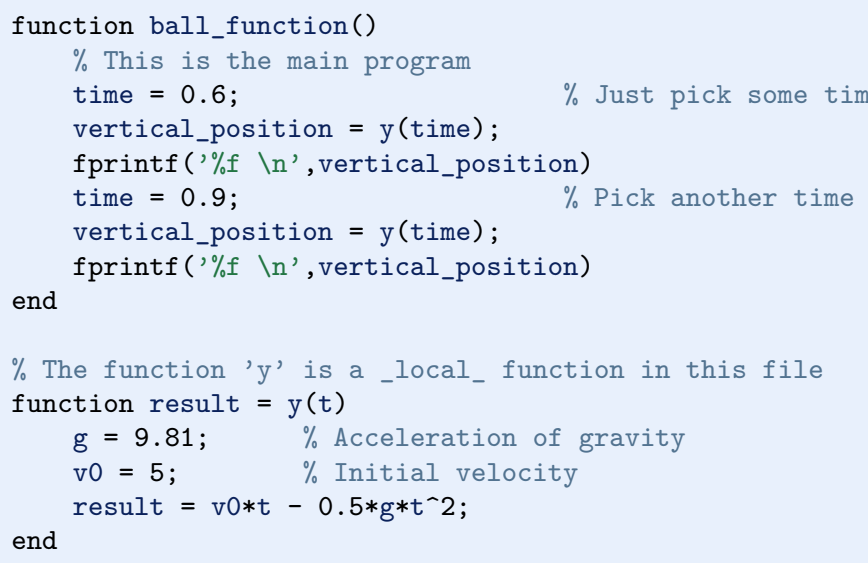

Here, Matlab interprets this as the definition of two functions, recognized by the reserved word function that appears two places. The first function ball_function, is defined by the statements between (and including) function 
ball_function() and the first end. Note that the first function in a file should have the same name as the name of the file (apart from the extension .m). The second function, i.e. $y$, is similarly defined between function result $=y(t)$ and the second end.

Opposed to the function y, the function ball_function does not return a value. This is stated in the first line of each function definition. Comparing, you notice that y has an assignment there, whereas ball_function has not. The final statement of the function y, i.e.

result $=\mathrm{v} 0 * \mathrm{t}-0.5 * \mathrm{~g} * \mathrm{t}^{\wedge} 2$

will be understood by Matlab as "first compute the expression, then place the result in result and send it back (i.e. return) to where the function was called from". The function depends on one variable (or we say that it takes one argument or input parameter), the value of which must be provided when the function is called.

What do these things mean? Well, the function definition itself, e.g. of y, just tells Matlab that there is a function y, taking the specified arguments as input, and returning a specified output result. Matlab keeps this information ready for use in case a call to y is performed elsewhere in the code. In our case, a call to y happens twice by the line vertical_position $=y($ time $)$. By this instruction, Matlab takes y (time) as a call to the function $y$, assigning the value of time to the variable $t$. So in the first call, $t$ becomes 0.6 , while in the second call $t$ becomes 0.9 . In both cases the code lines of y are executed and the returned result (the output parameter) is stored in vertical_position, before it is next printed by Matlab.

Note that the reserved word return may be used to enforce a return from a function before it reaches the end. For example, if a function contains if-elseif-else constructions, return may be done from within any of the branches. This may be illustrated by the following function containing three return statements:

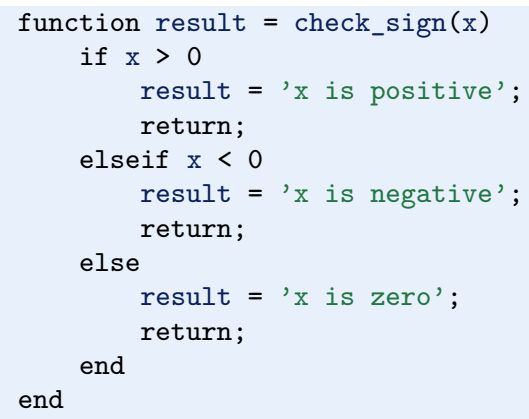

Remember that only one of the branches is executed for a single call on check sign, so depending on the number $\mathrm{x}$, the return may take place from any of the three return alternatives.

One phrase you will meet often when dealing with programming, is main program or main function, or that some code is in main. This is nothing particular to Matlab, and simply means the first function that is defined in a file, e.g. 
ball_function above. You may define as many functions as you like in a file after the main function. These then become local functions, i.e. they are only known inside that file. In particular, only the main function may be called from the command window, whereas local functions may not.

A function may take no arguments, or many, in which case they are just listed within the parentheses (following the function name) and separated by a comma. Let us illustrate. Take a slight variation of the ball example and assume that the ball is not thrown straight up, but at an angle, so that two coordinates are needed to specify its position at any time. According to Newton's laws (when air resistance is negligible), the vertical position is given by $y(t)=v_{0 y} t-0.5 g t^{2}$ and the horizontal position by $x(t)=v_{0 x} t$. We can include both these expressions in a new version of our program that prints the position of the ball for chosen times. Assume we want to evaluate these expressions at two points in time, $t=0.6 \mathrm{~s}$ and $t=0.9 \mathrm{~s}$. We can pick some numbers for the initial velocity components v0y and v0x, name the program ball_position_xy.m, and write it for example as

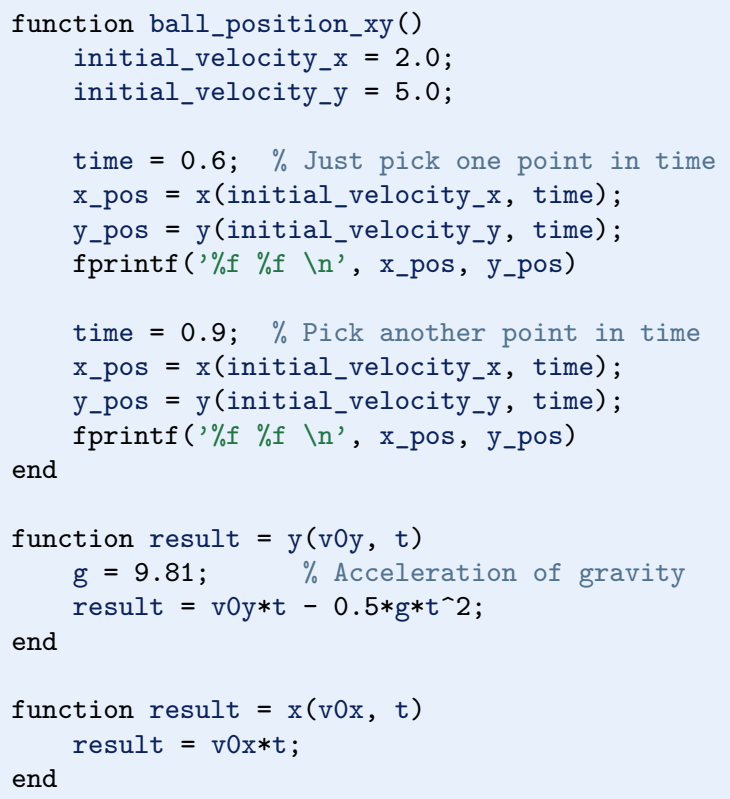

Now we compute and print the two components for the position, for each of the two chosen points in time. Notice how each of the two functions now takes two arguments. Running the program gives the output
1.21 .2342
$\begin{array}{ll}1.8 & 0.52695\end{array}$

A function may also return more than one value. For example, the two functions we just defined could alternatively have been defined into one as 


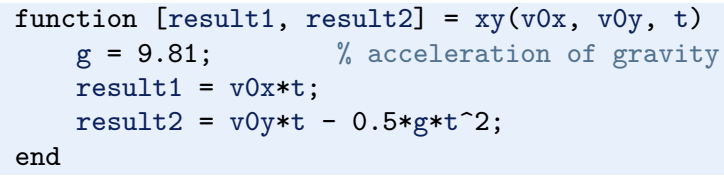

Notice the two return values result1 and result2 that are listed in the function header, i.e., the first line of the function definition. When calling the function, arguments must appear in the same order as in the function definition. We would then write

$$
\text { [x_pos,y_pos }]=x y(\text { initial__x_velocity, initial_y_velocity, time); }
$$

The variables $\mathrm{x}_{-}$pos and $\mathrm{y}_{-}$pos could then have been printed or used in other ways in the code.

There are possibilities for having a variable number of function input and output parameters (using nargin and nargout). However, we do not go further into that topic here.

Variables that are defined inside a function, e.g., $g$ in the last xy function, are local variables. This means they are only known inside the function. Therefore, if you had accidentally used $g$ in some calculation outside the function, you would have got an error message. By use of the reserved word global, a variable may be known also outside the function in which it is defined (without transferring it as a parameter). For example, if, in some function A, we write

global some_variable;

some_variable $=2$;

then, in another function B, we could use some_variable directly if we just specify it first as being global, e.g.

global some_variable;

some_other_variable = some_variable $* 2$;

We could even change the value of some_variable itself inside B if we like, so that upon return to the function A, some_variable would have a new value. If you define one global and one local variable, both with the same name, the function only sees the local one, so the global variable is not affected by what happens with its local companion of the same name. The arguments named in the header of a function definition are by rule local variables inside the function. One should strive to define variables mostly where they are needed and not everywhere.

In any programming language, it is a good habit to include a little explanation of what the function is doing, unless what is done by the function is obvious, e.g., when having only a few simple code lines. This explanation (sometimes known as a doc string) should be placed just at the top of the function. This explanation is meant for a human who wants to understand the code, so it should say something about the purpose of the code and possibly explain the arguments and return values if needed. If we do that with our xy function from above, we may write the first lines of the function as 
function $x y(v 0 x, v 0 y, t)$

$\%$ Compute the $\mathrm{x}$ and $\mathrm{y}$ position of the ball at time $\mathrm{t}$

Note that a function you have written may call another function you have written, even if they are not defined within the same file. Such a call requires the called function to be located in a file with the same name as the function (apart from the extension .m). This file must also be located in a folder where Matlab can find it, e.g. in the same folder as the calling function.

Functions are straightforwardly passed as arguments to other functions, as illustrated by the following script function_as_argument.m:

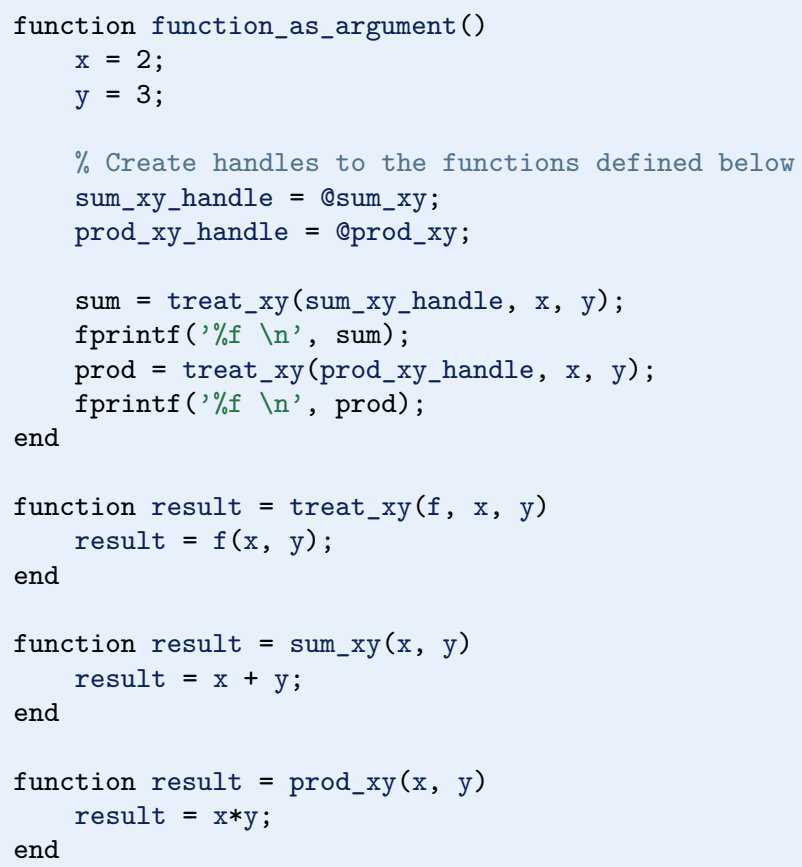

When run, this program first prints the sum of $x$ and $y$ (i.e., 5), and then it prints the product (i.e., 6). We see that treat_xy takes a function name as its first parameter. Inside treat_xy, that function is used to actually call the function that was given as input parameter. Therefore, as shown, we may call treat_xy with either sum_xy or prod_xy, depending on whether we want the sum or product of $x$ and $y$ to be calculated.

To transfer a function to the function treat_xy, we must use function handles, one for each function we want to transfer. This is done by the sign @ combined with the function name, as illustrated by the lines 
Note that it is the handle that is used in the function call, as, e.g., in

sum $=$ treat_xy $($ sum_xy_handle, $\mathrm{x}, \mathrm{y}) ;$

Functions may also be defined within other functions. It that case, they become local functions, or nested functions, known only to the function inside which they are defined. Functions defined in main are referred to as global functions. A nested function has full access to all variables in the parent function, i.e. the function within which it is defined.

One convenient way of defining one-line functions (they can not be more than one line!), is by use of anonymous functions. You may then define, e.g., a square function by the name my_square, as

my_square $=@(x) x^{\wedge} 2$;

and then use it simply as

$\mathrm{y}=$ my_sqare $(2)$;

which would have assigned the value 4 to $y$. Note that my_square here becomes a handle that may be used directly as a function parameter for example.

Overhead of function calls

Function calls have the downside of slowing down program execution. Usually, it is a good thing to split a program into functions, but in very computing intensive parts, e.g., inside long loops, one must balance the convenience of calling a function and the computational efficiency of avoiding function calls. It is a good rule to develop a program using plenty of functions and then in a later optimization stage, when everything computes correctly, remove function calls that are quantified to slow down the code.

\subsection{For Loops}

Many computations are repetitive by nature and programming languages have certain loop structures to deal with this. Here we will present what is referred to as a for loop (another kind of loop is a while loop, to be presented afterwards). Assume you want to calculate the square of each integer from 3 to 7 . This could be done with the following program.

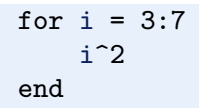

What happens when Matlab interprets your code here? First of all, the word for is a reserved word signalling to Matlab that a for loop is wanted. Matlab then sticks to the rules covering such constructions and understands that, in the present 
example, the loop should run 5 successive times (i.e., 5 iterations should be done), letting the variable $i$ take on the numbers $3,4,5,6,7$ in turn. During each iteration, the statement inside the loop (i.e. $i^{\sim} 2$ ) is carried out. After each iteration, $i$ is automatically (behind the scene) updated. When the last number is reached, the last iteration is performed and the loop is finished. When executed, the program will therefore print out $9,16,25,36$ and 49 . The variable $i$ is often referred to as a loop index, and its name (here $i$ ) is a choice of the programmer.

Note that, had there been several statements within the loop, they would all be executed with the same value of $i$ (before $i$ changed in the next iteration). Make sure you understand how program execution flows here, it is important.

The specification of the values desired for the loop variable (here $3: 7$ ) is more generally given as start:step:stop, meaning that the loop variable should take on the integers from start to stop, inclusive at both ends, in steps of step. If step is skipped, the default value is 1 , as in the example above. Note that decreasing integers may be produced by letting start > stop combined with a negative step. This makes it easy to, e.g., traverse arrays in either direction.

Let us modify ball_plot.m from Sect. 1.4 to illustrate how useful for loops are if you need to traverse arrays. In that example we computed the height of the ball at every milli-second during the first second of its (vertical) flight and plotted the height versus time.

Assume we want to find the maximum height during that time, how can we do it with a computer program? One alternative may be to compute all the thousand heights, store them in an array, and then run through the array to pick out the maximum. The program, named ball_max_height.m, may look as follows.

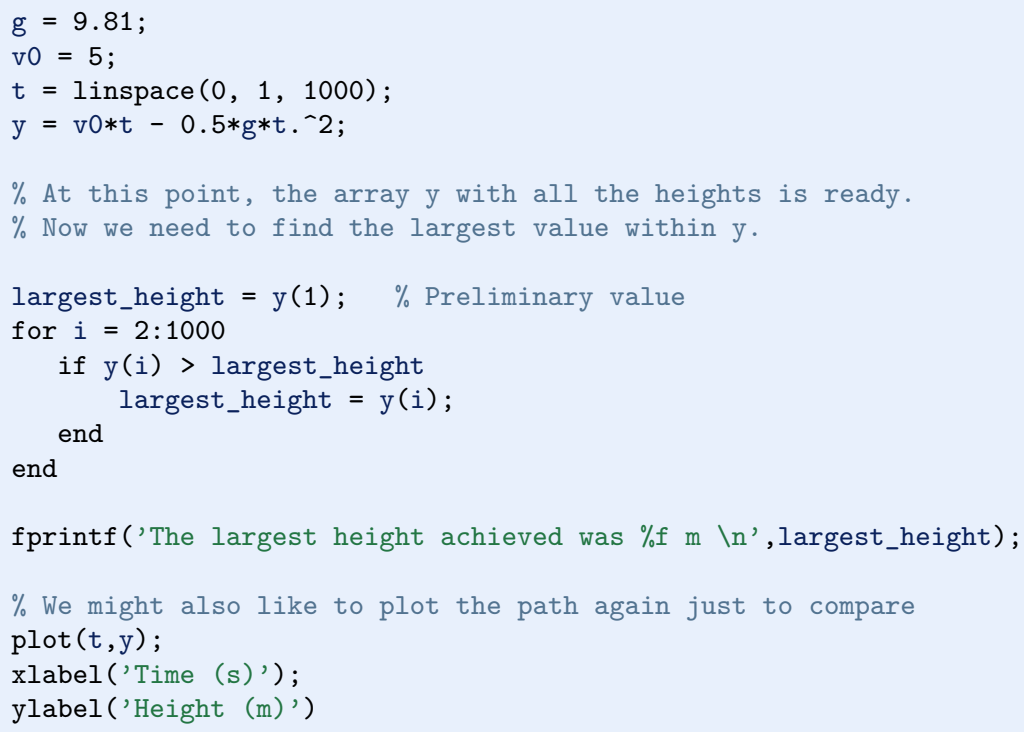

There is nothing new here, except the for loop construction, so let us look at it in more detail. As explained above, Matlab understands that a for loop is desired when it sees the word for. The value in $\mathrm{y}(1)$ is used as the preliminary largest 
height, so that, e.g., the very first check that is made is testing whether y(2) is larger than this height. If so, $\mathrm{y}(2)$ is stored as the largest height. The for loop then updates i to 2, and continues to check y (3), and so on. Each time we find a larger number, we store it. When finished, largest_height will contain the largest number from the array y. When you run the program, you get

The largest height achieved was $1.274210 \mathrm{~m}$

which compares favorably to the plot that pops up.

To implement the traversing of arrays with loops and indices, is sometimes challenging to get right. You need to understand the start, stop and step length choices for an index, and also how the index should enter expressions inside the loop. At the same time, however, it is something that programmers do often, so it is important to develop the right skills on these matters.

Having one loop inside another, referred to as a double loop, is sometimes useful, e.g., when doing linear algebra. Say we want to find the maximum among the numbers stored in a $4 \times 4$ matrix A. The code fragment could look like

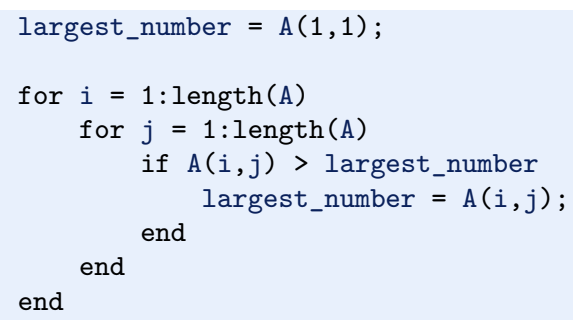

Here, all the $j$ indices $(1-4)$ will be covered for each value of index $i$. First, $i$ stays fixed at $i=1$, while $j$ runs over all its indices. Then, $i$ stays fixed at $i=2$ while $j$ runs over all its indices again, and so on. Sketch $A$ on a piece of paper and follow the first few loop iterations by hand, then you will realize how the double loop construction works. Using two loops is just a special case of using multiple or nested loops, and utilizing more than two loops is just a straightforward extension of what was shown here. Note, however, that the loop index name in multiple loops must be unique to each of the nested loops. Note also that each nested loop may have as many code lines as desired, both before and after the next inner loop.

The vectorized computation of heights that we did in ball_plot.m (Sect. 1.4) could alternatively have been done by traversing the time array ( $t$ ) and, for each $t$ element, computing the height according to the formula $y=v_{0} t-\frac{1}{2} g t^{2}$. However, it is important to know that vectorization goes much quicker. So when speed is important, vectorization is valuable.

Use loops to compute sums

One important use of loops, is to calculate sums. As a simple example, assume some variable $x$ given by the mathematical expression

$$
x=\sum_{i=1}^{N} 2 \cdot i,
$$


i.e., summing up the $N$ first even numbers. For some given $N$, say $N=5, x$ would typically be computed in a computer program as:

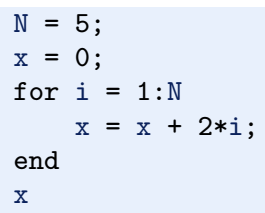

Executing this code will print the number 30 to the screen. Note in particular how the accumulation variable $\mathrm{x}$ is initialized to zero. The value of $\mathrm{x}$ then gets updated with each iteration of the loop, and not until the loop is finished will $\mathrm{x}$ have the correct value. This way of building up the value is very common in programming, so make sure you understand it by simulating the code segment above by hand. It is a technique used with loops in any programming language.

\subsection{While Loops}

Matlab also has another standard loop construction, the while loop, doing iterations with a loop index very much like the for loop. To illustrate what such a loop may look like, we consider another modification of ball_plot.m in Sect. 1.4. We will now change it so that it finds the time of flight for the ball. Assume the ball is thrown with a slightly lower initial velocity, say $4.5 \mathrm{~m} \mathrm{~s}^{-1}$, while everything else is kept unchanged. Since we still look at the first second of the flight, the heights at the end of the flight become negative. However, this only means that the ball has fallen below its initial starting position, i.e., the height where it left the hand, so there is no problem with that. In our array y we will then have a series of heights which towards the end of y become negative. Let us, in a program named ball_time.m find the time when heights start to get negative, i.e., when the ball crosses $y=0$. The program could look like this

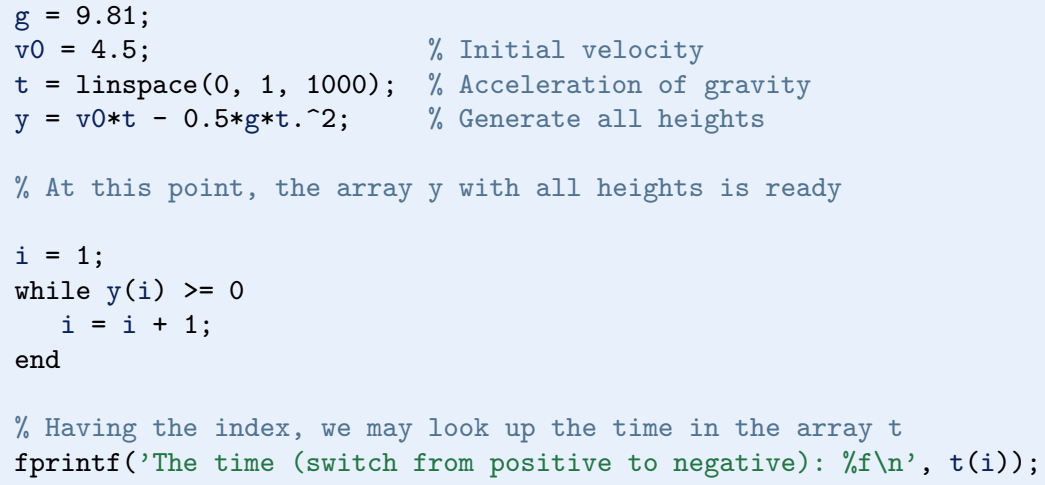


$\%$ We plot the path again just for comparison

$\operatorname{plot}(t, y)$;

xlabel ('Time (s)');

ylabel ('Height (m)');

If you type and run this program you should get

$y=0$ at 0.917417417417

The new thing here is the while loop only. The loop will run as long as the boolean expression $y(i)>=0$ evaluates to true. Note that the programmer introduced a variable (the loop index) by the name $i$, initialized it ( $i=1$ ) before the loop, and updated it $(i=i+1)$ in the loop. So for each iteration, $i$ is explicitly increased by 1 , allowing a check of successive elements in the array y.

Compared to a for loop, the programmer does not have to specify the number of iterations when coding a while loop. It simply runs until the boolean expression becomes false. Thus, a loop index (as we have in a for loop) is not required. Furthermore, if a loop index is used in a while loop, it is not increased automatically; it must be done explicitly by the programmer. Of course, just as in for loops and if blocks, there might be (arbitrarily) many code lines in a while loop. Any for loop may also be implemented as a while loop, but while loops are more general so not all of them can be expressed as a for loop.

A problem to be aware of, is what is usually referred to as an infinite loop. In those unintentional (erroneous) cases, the boolean expression of the while test never evaluates to false, and the program can not escape the loop. This is one of the most frequent errors you will experience as a beginning programmer. If you accidentally enter an infinite loop and the program just hangs forever, press $\mathrm{Ctrl}+\mathrm{c}$ to stop the program.

\subsection{Reading from and Writing to Files}

Input data for a program often come from files and the results of the computations are often written to file. To illustrate basic file handling, we consider an example where we read $x$ and $y$ coordinates from two columns in a file, apply a function $f$ to the $y$ coordinates, and write the results to a new two-column data file. The first line of the input file is a heading that we can just skip:

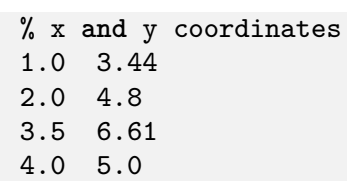

The relevant Matlab lines for reading the numbers and writing out a similar file are given in the file file_handling.m 


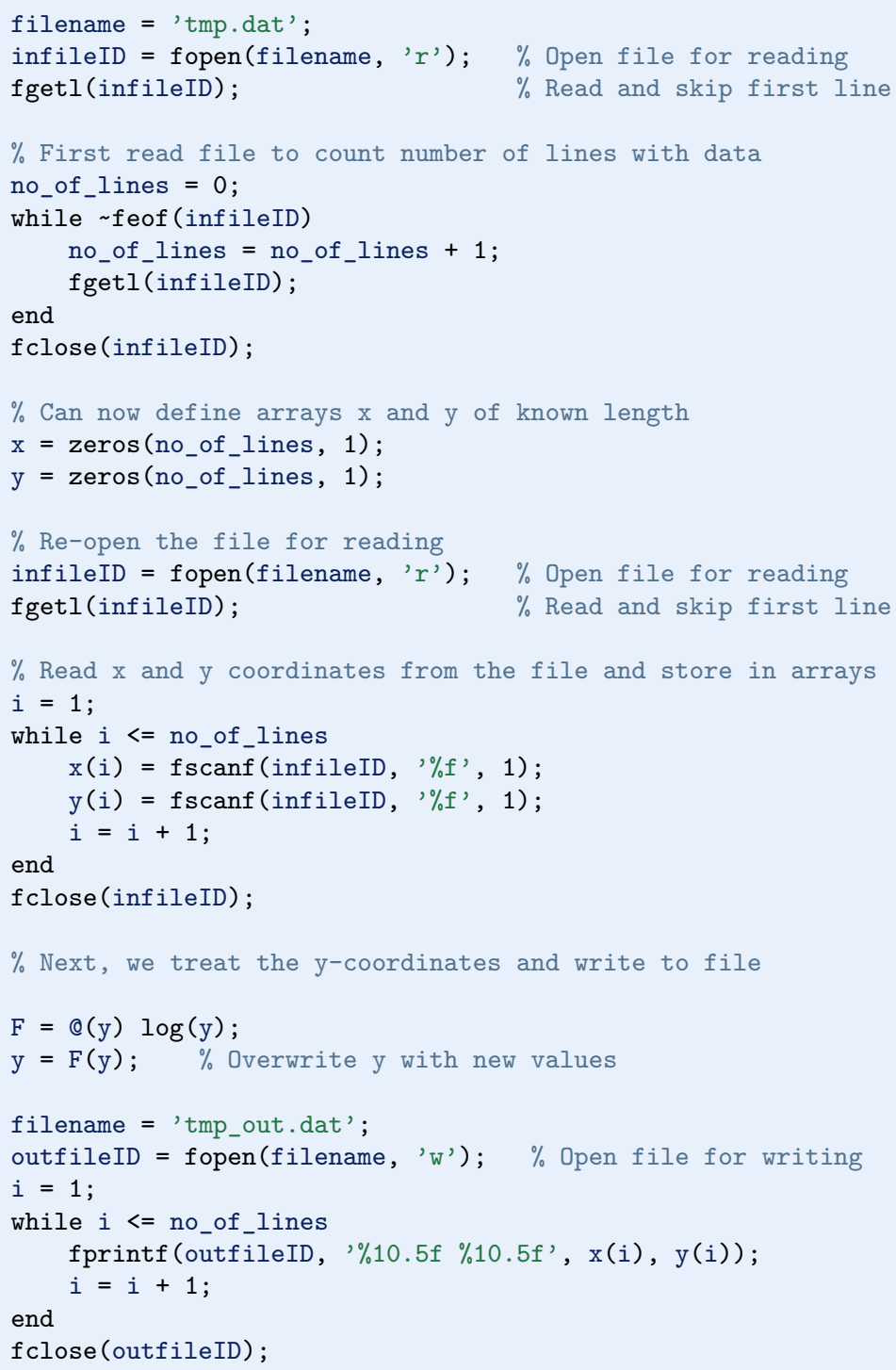

Such a file with a comment line and numbers in tabular format is very common so Matlab has functionality to ease reading and writing. Here is the same example (file file_handling_easy.m):

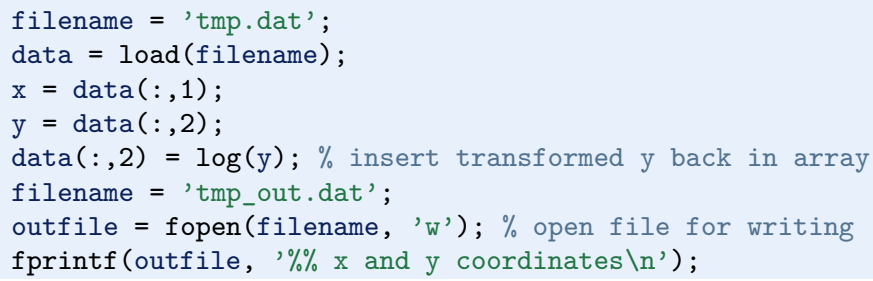


fprintf(outfile, , $\% 10.5 f \% 10.5 f \backslash n^{\prime}$, data);

fclose(outfile);

\subsection{Exercises}

\section{Exercise 2.1: Introducing errors}

Write the program ball_function.m as given in the text and confirm that the program runs correctly. Then save a copy of the program and use that program during the following error testing.

You are supposed to introduce errors in the code, one by one. For each error introduced, save and run the program, and comment how well Matlab's response corresponds to the actual error. When you are finished with one error, re-set the program to correct behavior (and check that it works!) before moving on to the next error.

a) Change the first line from function ball_function() to ball_ function(), i.e. remove the word function.

b) Change the first line from function ball_function() to function ball_ func (), i.e., change the name of the function.

c) Change the line function result $=y(t)$ to function $y(t)$.

d) Change the line function result $=y(t)$ to function result $=y()$, i.e., remove the parameter $t$.

e) Change the first statement that calls y from vertical_position $=y$ (time); to vertical_position $=y()$;

Filename: introducing_errors.m.

\section{Exercise 2.2: Compare integers a and $b$}

Explain briefly, in your own words, what the following program does.

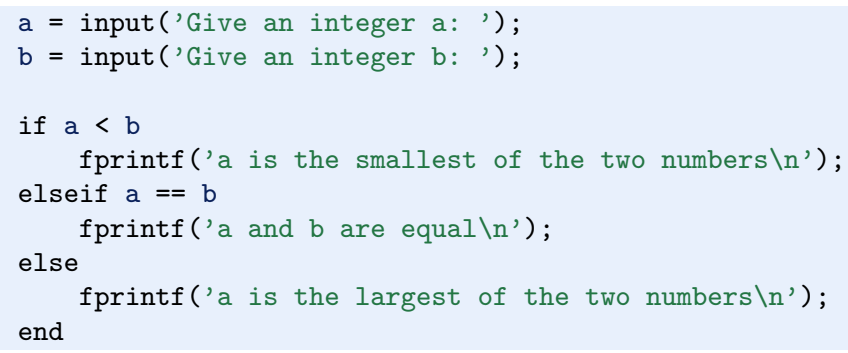

Proceed by writing the program, and then run it a few times with different values for $\mathrm{a}$ and $\mathrm{b}$ to confirm that it works as intended. In particular, choose combinations for $\mathrm{a}$ and $\mathrm{b}$ so that all three branches of the if construction get tested.

Filename: compare_a_and_b.m.

\section{Exercise 2.3: Functions for circumference and area of a circle}

Write a program that takes a circle radius $r$ as input from the user and then computes the circumference $\mathrm{C}$ and area $\mathrm{A}$ of the circle. Implement the computations of $\mathrm{C}$ and 
A as two separate functions that each takes $r$ as input parameter. Print $\mathrm{C}$ and A to the screen along with an appropriate text. Run the program with $r=1$ and confirm that you get the right answer.

Filename: functions_circumference_area.m.

\section{Exercise 2.4: Function for area of a rectangle}

Write a program that computes the area $A=b c$ of a rectangle. The values of $b$ and $c$ should be user input to the program. Also, write the area computation as a function that takes $b$ and $c$ as input parameters and returns the computed area. Let the program print the result to screen along with an appropriate text. Run the program with $b=2$ and $c=3$ to confirm correct program behavior.

Filename: function_area_rectangle.m.

\section{Exercise 2.5: Area of a polygon}

One of the most important mathematical problems through all times has been to find the area of a polygon, especially because real estate areas often had the shape of polygons, and it was necessary to pay tax for the area. We have a polygon as depicted below.

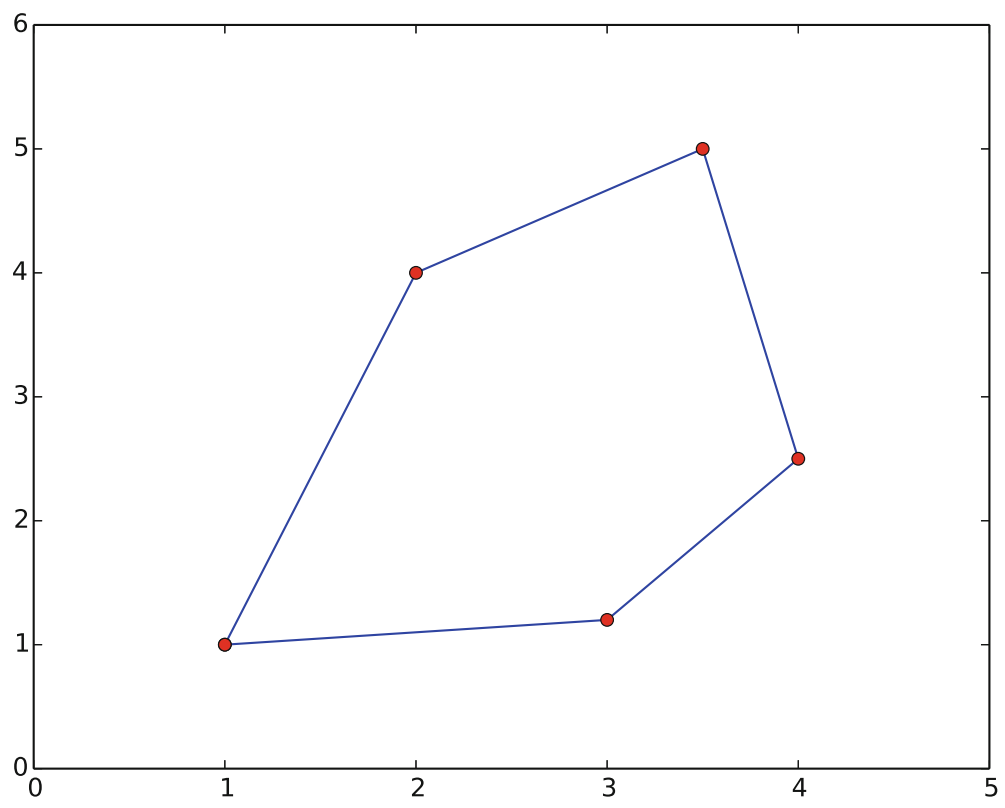

The vertices ("corners") of the polygon have coordinates $\left(x_{1}, y_{1}\right),\left(x_{2}, y_{2}\right), \ldots$, $\left(x_{n}, y_{n}\right)$, numbered either in a clockwise or counter clockwise fashion. The area $A$ of the polygon can amazingly be computed by just knowing the boundary coordinates:

$$
\begin{aligned}
A=\frac{1}{2} \mid & \mid\left(x_{1} y_{2}+x_{2} y_{3}+\ldots+x_{n-1} y_{n}+x_{n} y_{1}\right) \\
& -\left(y_{1} x_{2}+y_{2} x_{3}+\ldots+y_{n-1} x_{n}+y_{n} x_{1}\right) \mid .
\end{aligned}
$$


Write a function polyarea $(\mathrm{x}, \mathrm{y})$ that takes two coordinate arrays with the vertices as arguments and returns the area. Assume that $\mathrm{x}$ and $\mathrm{y}$ are either lists or arrays.

Test the function on a triangle, a quadrilateral, and a pentagon where you can calculate the area by alternative methods for comparison.

Filename: polyarea.m.

\section{Exercise 2.6: Average of integers}

Write a program that gets an integer $N>1$ from the user and computes the average of all integers $i=1, \ldots, N$. The computation should be done in a function that takes $N$ as input parameter. Print the result to the screen with an appropriate text. Run the program with $N=5$ and confirm that you get the correct answer.

Filename: average_1_to_N.m.

\section{Exercise 2.7: While loop with errors}

Assume some program has been written for the task of adding all integers $i=$ $1,2, \ldots, 10$ :

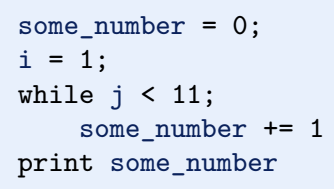

a) Identify the errors in the program by just reading the code and simulating the program by hand.

b) Write a new version of the program with errors corrected. Run this program and confirm that it gives the correct output.

Filename: while_loop_errors.m.

\section{Exercise 2.8: Area of rectangle versus circle}

Consider one circle and one rectangle. The circle has a radius $r=10.6$. The rectangle has sides $a$ and $b$, but only $a$ is known from the outset. Let $a=1.3$ and write a program that uses a while loop to find the largest possible integer $b$ that gives a rectangle area smaller than, but as close as possible to, the area of the circle. Run the program and confirm that it gives the right answer (which is $b=271$ ).

Filename: area_rectangle_vs_circle.m.

\section{Exercise 2.9: Find crossing points of two graphs}

Consider two functions $f(x)=x$ and $g(x)=x^{2}$ on the interval $[-4,4]$.

Write a program that, by trial and error, finds approximately for which values of $x$ the two graphs cross, i.e., $f(x)=g(x)$. Do this by considering $N$ equally distributed points on the interval, at each point checking whether $|f(x)-g(x)|<\epsilon$, where $\epsilon$ is some small number. Let $N$ and $\epsilon$ be user input to the program and let the result be printed to screen. Run your program with $N=400$ and $\epsilon=0.01$. Explain the output from the program. Finally, try also other values of $N$, keeping the value of $\epsilon$ fixed. Explain your observations.

Filename: crossing_2_graphs.m. 


\section{Exercise 2.10: Sort array with numbers}

The built-in function rand may be used to draw pseudo-random numbers for the standard uniform distribution between 0 and 1 (exclusive at both ends). See help rand.

Write a script that generates an array of 6 random numbers between 0 and 10 . The program should then sort the array so that numbers appear in increasing order. Let the program make a formatted print of the array to the screen both before and after sorting. The printouts should appear on the screen so that comparison is made easy. Confirm that the array has been sorted correctly.

Filename: sort_numbers.m.

\section{Exercise 2.11: Compute $\pi$}

Up through history, great minds have developed different computational schemes for the number $\pi$. We will here consider two such schemes, one by Leibniz (16461716), and one by Euler (1707-1783).

The scheme by Leibniz may be written

$$
\pi=8 \sum_{k=0}^{\infty} \frac{1}{(4 k+1)(4 k+3)},
$$

while one form of the Euler scheme may appear as

$$
\pi=\sqrt{6 \sum_{k=1}^{\infty} \frac{1}{k^{2}}}
$$

If only the first $N$ terms of each sum are used as an approximation to $\pi$, each modified scheme will have computed $\pi$ with some error.

Write a program that takes $N$ as input from the user, and plots the error development with both schemes as the number of iterations approaches $N$. Your program should also print out the final error achieved with both schemes, i.e. when the number of terms is $\mathrm{N}$. Run the program with $N=100$ and explain briefly what the graphs show.

Filename: compute_pi.m.

\section{Exercise 2.12: Compute combinations of sets}

Consider an ID number consisting of two letters and three digits, e.g., RE198. How many different numbers can we have, and how can a program generate all these combinations?

If a collection of $n$ things can have $m_{1}$ variations of the first thing, $m_{2}$ of the second and so on, the total number of variations of the collection equals $m_{1} m_{2} \cdots m_{n}$. In particular, the ID number exemplified above can have $26 \cdot 26 \cdot 10 \cdot 10 \cdot 10=676,000$ variations. To generate all the combinations, we must have five nested for loops. The first two run over all letters $\mathrm{A}, \mathrm{B}$, and so on to $\mathrm{Z}$, while the next three run over all digits $0,1, \ldots, 9$.

To convince yourself about this result, start out with an ID number on the form A3 where the first part can vary among A, B, and C, and the digit can be among 1, 2 , or 3 . We must start with A and combine it with 1,2, and 3, then continue with 
B, combined with 1,2 , and 3 , and finally combine $\mathrm{C}$ with 1,2 , and 3 . A double for loop does the work.

a) In a deck of cards, each card is a combination of a rank and a suit. There are 13 ranks: ace (A), 2, 3, 4, 5, 6, 7, 8, 9, 10, jack (J), queen (Q), king (K), and four suits: clubs (C), diamonds (D), hearts (H), and spades (S). A typical card may be D3. Write statements that generate a deck of cards, i.e., all the combinations $\mathrm{CA}, \mathrm{C} 2, \mathrm{C} 3$, and so on to SK.

b) A vehicle registration number is on the form DE562, where the letters vary from $\mathrm{A}$ to $\mathrm{Z}$ and the digits from 0 to 9 . Write statements that compute all the possible registration numbers and stores them in a list.

c) Generate all the combinations of throwing two dice (the number of eyes can vary from 1 to 6 ). Count how many combinations where the sum of the eyes equals 7.

Filename: combine_sets.m.

\section{Exercise 2.13: Frequency of random numbers}

Write a program that takes a positive integer $N$ as input and then draws $N$ random integers in the interval $[1,6]$ (both ends inclusive). In the program, count how many of the numbers, $M$, that equal 6 and write out the fraction $M / N$. Also, print all the random numbers to the screen so that you can check for yourself that the counting is correct. Run the program with a small value for $\mathrm{N}$ (e.g., $\mathrm{N}=10$ ) to confirm that it works as intended.

Hint Use $1+f$ loor $(6 *$ rand ()$)$ to draw a random integer between 1 and 6 .

Filename: count_random_numbers.m.

Remarks For large $N$, this program computes the probability $M / N$ of getting six eyes when throwing a dice.

\section{Exercise 2.14: Game 21}

Consider some game where each participant draws a series of random integers evenly distributed from 0 and 10, with the aim of getting the sum as close as possible to 21, but not larger than 21. You are out of the game if the sum passes 21 . After each draw, you are told the number and your total sum, and are asked whether you want another draw or not. The one coming closest to 21 is the winner.

Implement this game in a program.

Hint Use floor $(11 * r$ and ()$)$ to draw random integers in $[0,10]$.

Filename: game_21.m.

\section{Exercise 2.15: Linear interpolation}

Some measurements $y_{i}, i=0,1, \ldots, N$ (given below), of a quantity $y$ have been collected regularly, once every minute, at times $t_{i}=i, i=0,1, \ldots, N$. We want to find the value $y$ in between the measurements, e.g., at $t=3.2 \mathrm{~min}$. Computing such $y$ values is called interpolation. 
Let your program use linear interpolation to compute $y$ between two consecutive measurements:

1. Find $i$ such that $t_{i} \leq t \leq t_{i+1}$.

2. Find a mathematical expression for the straight line that goes through the points $\left(i, y_{i}\right)$ and $\left(i+1, y_{i+1}\right)$.

3. Compute the $y$ value by inserting the user's time value in the expression for the straight line.

a) Implement the linear interpolation technique in a function that takes an array with the $y_{i}$ measurements as input, together with some time $t$, and returns the interpolated $y$ value at time $t$.

b) Write another function with a loop where the user is asked for a time on the interval $[0, N]$ and the corresponding (interpolated) $y$ value is written to the screen. The loop is terminated when the user gives a negative time.

c) Use the following measurements: 4.4,2.0,11.0,21.5, 7.5, corresponding to times $0,1, \ldots, 4(\mathrm{~min})$, and compute interpolated values at $t=2.5$ and $t=3.1 \mathrm{~min}$. Perform separate hand calculations to check that the output from the program is correct.

Filename: linear_interpolation.m.

\section{Exercise 2.16: Test straight line requirement}

Assume the straight line function $f(x)=4 x+1$. Write a script that tests the "point-slope" form for this line as follows. Within a chosen interval on the $x$-axis (for example, for $x$ between 0 and 10), randomly pick 100 points on the line and check if the following requirement is fulfilled for each point:

$$
\frac{f\left(x_{i}\right)-f(c)}{x_{i}-c}=a, \quad i=1,2, \ldots, 100,
$$

where $a$ is the slope of the line and $c$ defines a fixed point $(c, f(c))$ on the line. Let $c=2$ here.

Filename: test_straight_line.m.

\section{Exercise 2.17: Fit straight line to data}

Assume some measurements $y_{i}, i=1,2, \ldots, 5$ have been collected, once every second. Your task is to write a program that fits a straight line to those data.

a) Make a function that computes the error between the straight line $f(x)=a x+b$ and the measurements:

$$
e=\sum_{i=1}^{5}\left(a x_{i}+b-y_{i}\right)^{2}
$$

b) Make a function with a loop where you give $a$ and $b$, the corresponding value of $e$ is written to the screen, and a plot of the straight line $f(x)=a x+b$ together with the discrete measurements is shown. 
c) Given the measurements $0.5,2.0,1.0,1.5,7.5$, at times $0,1,2,3$, 4, use the function in b) to interactively search for $a$ and $b$ such that $e$ is minimized.

Filename: fit_straight_line.m.

Remarks Fitting a straight line to measured data points is a very common task. The manual search procedure in c) can be automated by using a mathematical method called the method of least squares.

\section{Exercise 2.18: Fit sines to straight line}

A lot of technology, especially most types of digital audio devices for processing sound, is based on representing a signal of time as a sum of sine functions. Say the signal is some function $f(t)$ on the interval $[-\pi, \pi]$ (a more general interval $[a, b]$ can easily be treated, but leads to slightly more complicated formulas). Instead of working with $f(t)$ directly, we approximate $f$ by the sum

$$
S_{N}(t)=\sum_{n=1}^{N} b_{n} \sin (n t),
$$

where the coefficients $b_{n}$ must be adjusted such that $S_{N}(t)$ is a good approximation to $f(t)$. We shall in this exercise adjust $b_{n}$ by a trial-and-error process.

a) Make a function sinesum( $t, \mathrm{~b})$ that returns $S_{N}(t)$, given the coefficients $b_{n}$ in an array $b$ and time coordinates in an array $t$. Note that if $t$ is an array, the return value is also an array.

b) Write a function test_sinesum() that calls sinesum( $t$, b) in a) and determines if the function computes a test case correctly. As test case, let $t$ be an array with values $-\pi / 2$ and $\pi / 4$, choose $N=2$, and $b_{1}=4$ and $b_{2}=-3$. Compute $S_{N}(t)$ by hand to get reference values.

c) Make a function plot_compare $(f, N, M)$ that plots the original function $f(t)$ together with the sum of sines $S_{N}(t)$, so that the quality of the approximation $S_{N}(t)$ can be examined visually. The argument $\mathrm{f}$ is a Matlab function implementing $f(t), \mathrm{N}$ is the number of terms in the sum $S_{N}(t)$, and $\mathrm{M}$ is the number of uniformly distributed $t$ coordinates used to plot $f$ and $S_{N}$.

d) Write a function error (b, $f, M)$ that returns a mathematical measure of the error in $S_{N}(t)$ as an approximation to $f(t)$ :

$$
E=\sqrt{\sum_{i}\left(f\left(t_{i}\right)-S_{N}\left(t_{i}\right)\right)^{2}},
$$

where the $t_{i}$ values are $M$ uniformly distributed coordinates on $[-\pi, \pi]$. The array b holds the coefficients in $S_{N}$ and $f$ is a Matlab function implementing the mathematical function $f(t)$.

e) Make a function trial $(f, N)$ for interactively giving $b_{n}$ values and getting a plot on the screen where the resulting $S_{N}(t)$ is plotted together with $f(t)$. The error in the approximation should also be computed as indicated in $\mathrm{d}$ ). The argument $\mathrm{f}$ is a Matlab function for $f(t)$ and $\mathrm{N}$ is the number of terms $N$ in the 
$\operatorname{sum} S_{N}(t)$. The trial function can run a loop where the user is asked for the $b_{n}$ values in each pass of the loop and the corresponding plot is shown. You must find a way to terminate the loop when the experiments are over. Use $M=500$ in the calls to plot_compare and error.

f) Choose $f(t)$ to be a straight line $f(t)=\frac{1}{\pi} t$ on $[-\pi, \pi]$. Call trial (f, 3 ) and try to find through experimentation some values $b_{1}, b_{2}$, and $b_{3}$ such that the sum of sines $S_{N}(t)$ is a good approximation to the straight line.

g) Now we shall try to automate the procedure in $\mathrm{f}$ ). Write a function that has three nested loops over values of $b_{1}, b_{2}$, and $b_{3}$. Let each loop cover the interval $[-1,1]$ in steps of 0.1 . For each combination of $b_{1}, b_{2}$, and $b_{3}$, the error in the approximation $S_{N}$ should be computed. Use this to find, and print, the smallest error and the corresponding values of $b_{1}, b_{2}$, and $b_{3}$. Let the program also plot $f$ and the approximation $S_{N}$ corresponding to the smallest error.

Filename: fit_sines.m.

\section{Remarks}

1. The function $S_{N}(x)$ is a special case of what is called a Fourier series. At the beginning of the 19th century, Joseph Fourier (1768-1830) showed that any function can be approximated analytically by a sum of cosines and sines. The approximation improves as the number of terms $(N)$ is increased. Fourier series are very important throughout science and engineering today.

(a) Finding the coefficients $b_{n}$ is solved much more accurately in Exercise 3.12, by a procedure that also requires much less human and computer work!

(b) In real applications, $f(t)$ is not known as a continuous function, but function values of $f(t)$ are provided. For example, in digital sound applications, music in a CD-quality WAV file is a signal with 44100 samples of the corresponding analog signal $f(t)$ per second.

\section{Exercise 2.19: Count occurrences of a string in a string}

In the analysis of genes one encounters many problem settings involving searching for certain combinations of letters in a long string. For example, we may have a string like

$$
\text { gene = 'AGTCAATGGAATAGGCCAAGCGAATATTTGGGCTACCA' }
$$

We may traverse this string letter by letter. The length of the string is given by length (gene), so with a loop index $i$, for $i=1$ : length (gene) will produce the required index values. Letter number $i$ is then reached through gene( $i)$, and a substring from index $i$ up to and including $j$, is created by gene $(i: j)$.

a) Write a function freq (letter, text) that returns the frequency of the letter letter in the string text, i.e., the number of occurrences of letter divided by the length of text. Call the function to determine the frequency of $\mathrm{C}$ and $\mathrm{G}$ in the gene string above. Compute the frequency by hand too.

b) Write a function pairs (letter, text) that counts how many times a pair of the letter letter (e.g., GG) occurs within the string text. Use the function 
to determine how many times the pair AA appears in the string gene above. Perform a manual counting too to check the answer.

c) Write a function mystruct (text) that counts the number of a certain structure in the string text. The structure is defined as $\mathrm{G}$ followed by $\mathrm{A}$ or $\mathrm{T}$ until a double GG. Perform a manual search for the structure too to control the computations by mystruct.

Filename: count_substrings.m.

Open Access This chapter is distributed under the terms of the Creative Commons AttributionNonCommercial 4.0 International License (http://creativecommons.org/licenses/by-nc/4.0/), which permits any noncommercial use, duplication, adaptation, distribution and reproduction in any medium or format, as long as you give appropriate credit to the original author(s) and the source, a link is provided to the Creative Commons license and any changes made are indicated.

The images or other third party material in this chapter are included in the work's Creative Commons license, unless indicated otherwise in the credit line; if such material is not included in the work's Creative Commons license and the respective action is not permitted by statutory regulation, users will need to obtain permission from the license holder to duplicate, adapt or reproduce the material. 


\section{Computing Integrals}

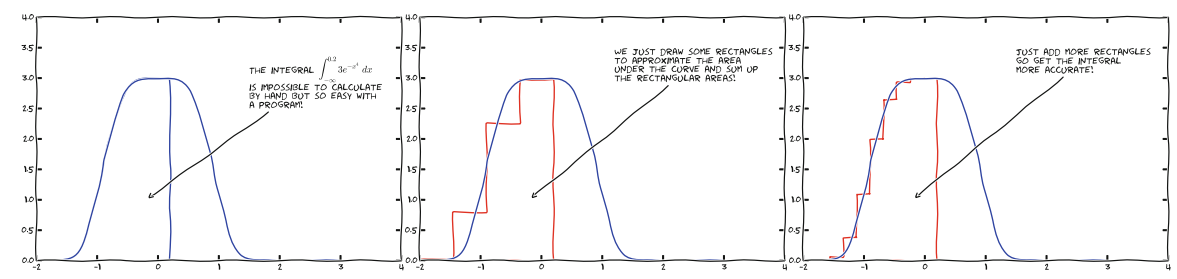

We now turn our attention to solving mathematical problems through computer programming. There are many reasons to choose integration as our first application. Integration is well known already from high school mathematics. Most integrals are not tractable by pen and paper, and a computerized solution approach is both very much simpler and much more powerful - you can essentially treat all integrals $\int_{a}^{b} f(x) d x$ in 10 lines of computer code (!). Integration also demonstrates the difference between exact mathematics by pen and paper and numerical mathematics on a computer. The latter approaches the result of the former without any worries about rounding errors due to finite precision arithmetics in computers (in contrast to differentiation, where such errors prevent us from getting a result as accurate as we desire on the computer). Finally, integration is thought of as a somewhat difficult mathematical concept to grasp, and programming integration should greatly help with the understanding of what integration is and how it works. Not only shall we understand how to use the computer to integrate, but we shall also learn a series of good habits to ensure your computer work is of the highest scientific quality. In particular, we have a strong focus on how to write Matlab code that is free of programming mistakes.

Calculating an integral is traditionally done by

$$
\int_{a}^{b} f(x) d x=F(b)-F(a)
$$


where

$$
f(x)=\frac{d F}{d x} .
$$

The major problem with this procedure is that we need to find the anti-derivative $F(x)$ corresponding to a given $f(x)$. For some relatively simple integrands $f(x)$, finding $F(x)$ is a doable task, but it can very quickly become challenging, even impossible!

The method (3.1) provides an exact or analytical value of the integral. If we relax the requirement of the integral being exact, and instead look for approximate values, produced by numerical methods, integration becomes a very straightforward task for any given $f(x)(!)$.

The downside of a numerical method is that it can only find an approximate answer. Leaving the exact for the approximate is a mental barrier in the beginning, but remember that most real applications of integration will involve an $f(x)$ function that contains physical parameters, which are measured with some error. That is, $f(x)$ is very seldom exact, and then it does not make sense to compute the integral with a smaller error than the one already present in $f(x)$.

Another advantage of numerical methods is that we can easily integrate a function $f(x)$ that is only known as samples, i.e., discrete values at some $x$ points, and not as a continuous function of $x$ expressed through a formula. This is highly relevant when $f$ is measured in a physical experiment.

\subsection{Basic Ideas of Numerical Integration}

We consider the integral

$$
\int_{a}^{b} f(x) d x .
$$

Most numerical methods for computing this integral split up the original integral into a sum of several integrals, each covering a smaller part of the original integration interval $[a, b]$. This re-writing of the integral is based on a selection of integration points $x_{i}, i=0,1, \ldots, n$ that are distributed on the interval $[a, b]$. Integration points may, or may not, be evenly distributed. An even distribution simplifies expressions and is often sufficient, so we will mostly restrict ourselves to that choice. The integration points are then computed as

$$
x_{i}=a+i h, \quad i=0,1, \ldots, n
$$

where

$$
h=\frac{b-a}{n} .
$$

Given the integration points, the original integral is re-written as a sum of integrals, each integral being computed over the sub-interval between two consecutive 
integration points. The integral in (3.2) is thus expressed as

$$
\int_{a}^{b} f(x) d x=\int_{x_{0}}^{x_{1}} f(x) d x+\int_{x_{1}}^{x_{2}} f(x) d x+\ldots+\int_{x_{n-1}}^{x_{n}} f(x) d x .
$$

Note that $x_{0}=a$ and $x_{n}=b$.

Proceeding from (3.5), the different integration methods will differ in the way they approximate each integral on the right hand side. The fundamental idea is that each term is an integral over a small interval $\left[x_{i}, x_{i+1}\right]$, and over this small interval, it makes sense to approximate $f$ by a simple shape, say a constant, a straight line, or a parabola, which we can easily integrate by hand. The details will become clear in the coming examples.

Computational example To understand and compare the numerical integration methods, it is advantageous to use a specific integral for computations and graphical illustrations. In particular, we want to use an integral that we can calculate by hand such that the accuracy of the approximation methods can easily be assessed. Our specific integral is taken from basic physics. Assume that you speed up your car from rest and wonder how far you go in $T$ seconds. The distance is given by the integral $\int_{0}^{T} v(t) d t$, where $v(t)$ is the velocity as a function of time. A rapidly increasing velocity function might be

$$
v(t)=3 t^{2} e^{t^{3}} .
$$

The distance after one second is

$$
\int_{0}^{1} v(t) d t
$$

which is the integral we aim to compute by numerical methods. Fortunately, the chosen expression of the velocity has a form that makes it easy to calculate the anti-derivative as

$$
V(t)=e^{t^{3}}-1 .
$$

We can therefore compute the exact value of the integral as $V(1)-V(0) \approx 1.718$ (rounded to 3 decimals for convenience).

\subsection{The Composite Trapezoidal Rule}

The integral $\int_{a}^{b} f(x) d x$ may be interpreted as the area between the $x$ axis and the graph $y=f(x)$ of the integrand. Fig. 3.1 illustrates this area for the choice (3.7). Computing the integral $\int_{0}^{1} f(t) d t$ amounts to computing the area of the hatched region.

If we replace the true graph in Fig. 3.1 by a set of straight line segments, we may view the area rather as composed of trapezoids, the areas of which are easy to 


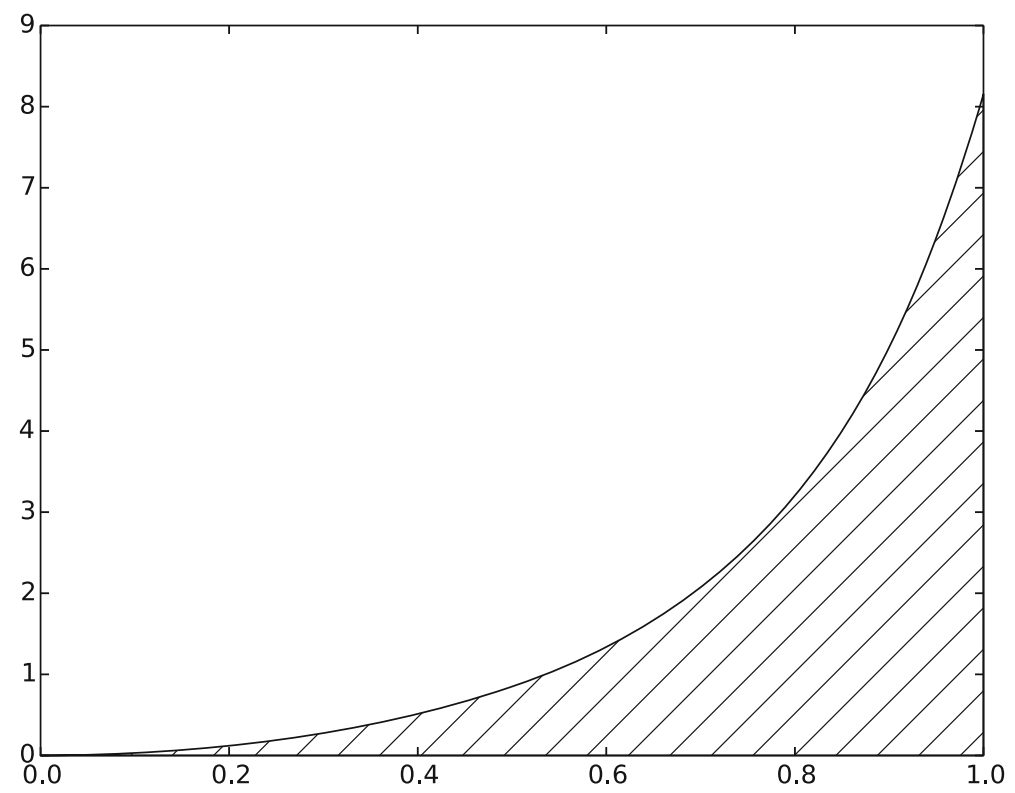

Fig. 3.1 The integral of $v(t)$ interpreted as the area under the graph of $v$

compute. This is illustrated in Fig. 3.2, where 4 straight line segments give rise to 4 trapezoids, covering the time intervals $[0,0.2),[0.2,0.6),[0.6,0.8)$ and $[0.8,1.0]$. Note that we have taken the opportunity here to demonstrate the computations with time intervals that differ in size.

The areas of the 4 trapezoids shown in Fig. 3.2 now constitute our approximation to the integral (3.7):

$$
\begin{aligned}
\int_{0}^{1} v(t) d t \approx & h_{1}\left(\frac{v(0)+v(0.2)}{2}\right)+h_{2}\left(\frac{v(0.2)+v(0.6)}{2}\right) \\
& +h_{3}\left(\frac{v(0.6)+v(0.8)}{2}\right)+h_{4}\left(\frac{v(0.8)+v(1.0)}{2}\right),
\end{aligned}
$$

where

$$
\begin{aligned}
& h_{1}=(0.2-0.0), \\
& h_{2}=(0.6-0.2), \\
& h_{3}=(0.8-0.6), \\
& h_{4}=(1.0-0.8)
\end{aligned}
$$

With $v(t)=3 t^{2} e^{t^{3}}$, each term in (3.9) is readily computed and our approximate computation gives

$$
\int_{0}^{1} v(t) d t \approx 1.895 .
$$




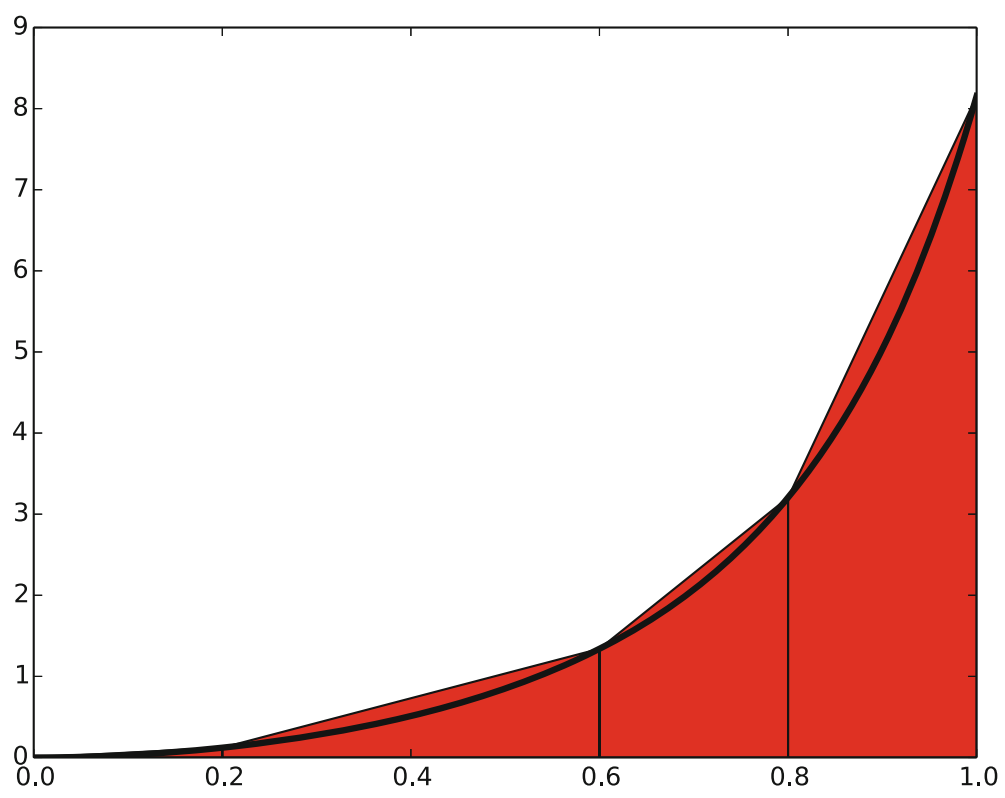

Fig. 3.2 Computing approximately the integral of a function as the sum of the areas of the trapezoids

Compared to the true answer of 1.718 , this is off by about $10 \%$. However, note that we used just 4 trapezoids to approximate the area. With more trapezoids, the approximation would have become better, since the straight line segments at the upper trapezoid side then would follow the graph more closely. Doing another hand calculation with more trapezoids is not too tempting for a lazy human, though, but it is a perfect job for a computer! Let us therefore derive the expressions for approximating the integral by an arbitrary number of trapezoids.

\subsubsection{The General Formula}

For a given function $f(x)$, we want to approximate the integral $\int_{a}^{b} f(x) d x$ by $n$ trapezoids (of equal width). We start out with (3.5) and approximate each integral on the right hand side with a single trapezoid. In detail,

$$
\begin{aligned}
\int_{a}^{b} f(x) d x= & \int_{x_{0}}^{x_{1}} f(x) d x+\int_{x_{1}}^{x_{2}} f(x) d x+\ldots+\int_{x_{n-1}}^{x_{n}} f(x) d x \\
\approx & h \frac{f\left(x_{0}\right)+f\left(x_{1}\right)}{2}+h \frac{f\left(x_{1}\right)+f\left(x_{2}\right)}{2}+\ldots \\
& +h \frac{f\left(x_{n-1}\right)+f\left(x_{n}\right)}{2}
\end{aligned}
$$


By simplifying the right hand side of (3.15) we get

$$
\int_{a}^{b} f(x) d x \approx \frac{h}{2}\left[f\left(x_{0}\right)+2 f\left(x_{1}\right)+2 f\left(x_{2}\right)+\ldots+2 f\left(x_{n-1}\right)+f\left(x_{n}\right)\right]
$$

which is more compactly written as

$$
\int_{a}^{b} f(x) d x \approx h\left[\frac{1}{2} f\left(x_{0}\right)+\sum_{i=1}^{n-1} f\left(x_{i}\right)+\frac{1}{2} f\left(x_{n}\right)\right]
$$

\section{Composite integration rules}

The word composite is often used when a numerical integration method is applied with more than one sub-interval. Strictly speaking then, writing, e.g., "the trapezoidal method", should imply the use of only a single trapezoid, while "the composite trapezoidal method" is the most correct name when several trapezoids are used. However, this naming convention is not always followed, so saying just "the trapezoidal method" may point to a single trapezoid as well as the composite rule with many trapezoids.

\subsubsection{Implementation}

Specific or general implementation? Suppose our primary goal was to compute the specific integral $\int_{0}^{1} v(t) d t$ with $v(t)=3 t^{2} e^{t^{3}}$. First we played around with a simple hand calculation to see what the method was about, before we (as one often does in mathematics) developed a general formula (3.17) for the general or "abstract" integral $\int_{a}^{b} f(x) d x$. To solve our specific problem $\int_{0}^{1} v(t) d t$ we must then apply the general formula (3.17) to the given data (function and integral limits) in our problem. Although simple in principle, the practical steps are confusing for many because the notation in the abstract problem in (3.17) differs from the notation in our special problem. Clearly, the $f, x$, and $h$ in (3.17) correspond to $v, t$, and perhaps $\Delta t$ for the trapezoid width in our special problem.

\section{The programmer's dilemma}

1. Should we write a special program for the special integral, using the ideas from the general rule (3.17), but replacing $f$ by $v, x$ by $t$, and $h$ by $\Delta t$ ?

2. Should we implement the general method (3.17) as it stands in a general function trapezoid $(\mathrm{f}, \mathrm{a}, \mathrm{b}, \mathrm{n}$ ) and solve the specific problem at hand by a specialized call to this function?

\section{Alternative 2 is always the best choice!}

The first alternative in the box above sounds less abstract and therefore more attractive to many. Nevertheless, as we hope will be evident from the examples, the second alternative is actually the simplest and most reliable from both a mathematical and programming point of view. These authors will claim that the second 
alternative is the essence of the power of mathematics, while the first alternative is the source of much confusion about mathematics!

Implementation with functions For the integral $\int_{a}^{b} f(x) d x$ computed by the formula (3.17) we want the corresponding Matlab function trapezoid to take any $f$, $a, b$, and $n$ as input and return the approximation to the integral.

We write a Matlab function trapezoidal in a file trapezoidal.m as close as possible to the formula (3.17), making sure variable names correspond to the mathematical notation:

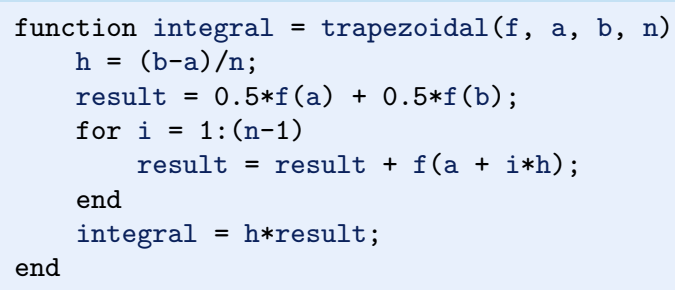

This function must be placed in a file trapezoidal . $\mathrm{m}$ to be reused in other programs and in interactive sessions.

Solving our specific problem in a session An interactive session can make use of the trapezoidal function in trapezoidal. $\mathrm{m}$ to solve our particular problem $\int_{0}^{1} v(t) d t$ :

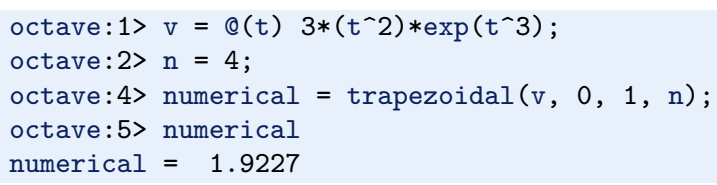

Let us compute the exact expression and the error in the approximation:

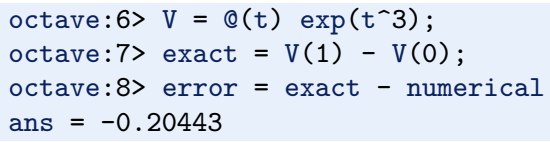

Is this error convincing? We can try a larger $n$ :

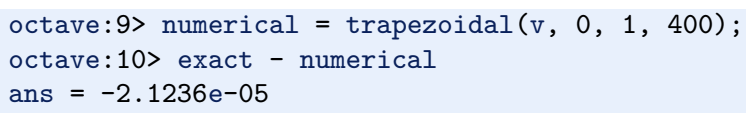

Fortunately, many more trapezoids give a much smaller error. 
Solving our specific problem in a program Instead of computing our special problem in an interactive session, we can do it in a program. As always, a chunk of code doing a particular thing is best isolated as a function even if we do not see any future reason to call the function several times and even if we have no need for arguments to parameterize what goes on inside the function. In the present case, we just put the statements we otherwise would have put in a main program, inside a function:

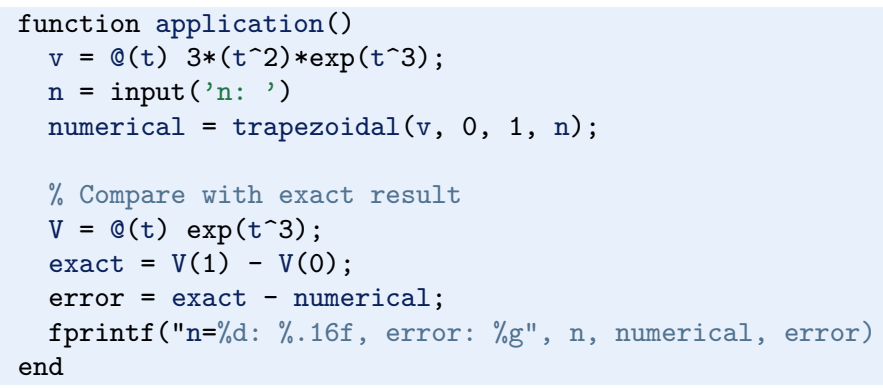

Now we compute our special problem by calling application() as the only statement in the main program. The application function and its call is in the file trapezoidal_app.m, which can be run as

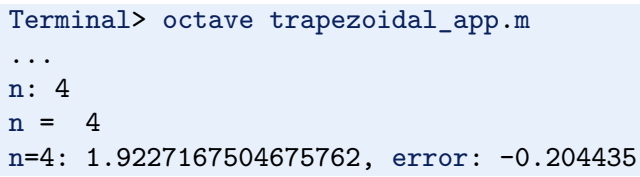

\subsubsection{Alternative Flat Special-Purpose Implementation}

Let us illustrate the implementation implied by alternative 1 in the Programmer's dilemma box in Sect. 3.2.2. That is, we make a special-purpose code where we adapt the general formula (3.17) to the specific problem $\int_{0}^{1} 3 t^{2} e^{t^{3}} d t$.

Basically, we use a for loop to compute the sum. Each term with $f(x)$ in the formula (3.17) is replaced by $3 t^{2} e^{t^{3}}, x$ by $t$, and $h$ by $\Delta t^{1}$. A first try at writing a plain, flat program doing the special calculation is

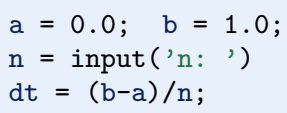

\footnotetext{
${ }^{1}$ Replacing $h$ by $\Delta t$ is not strictly required as many use $h$ as interval also along the time axis. Nevertheless, $\Delta t$ is an even more popular notation for a small time interval, so we adopt that common notation.
} 


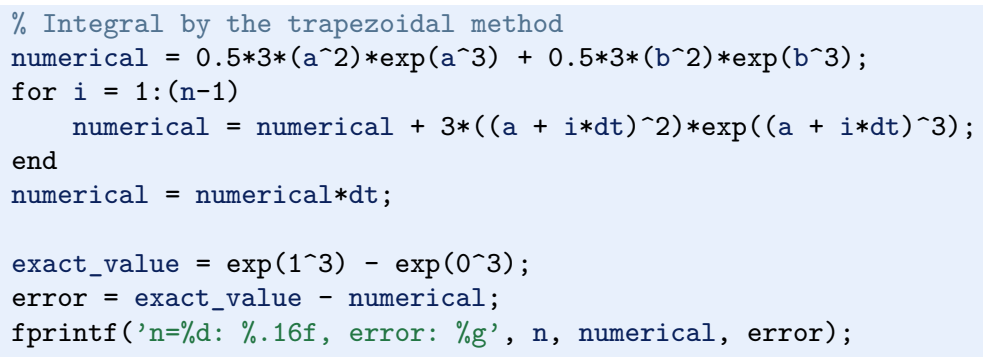

The problem with the above code is at least three-fold:

1. We need to reformulate (3.17) for our special problem with a different notation.

2. The integrand $3 t^{2} e^{t^{3}}$ is inserted many times in the code, which quickly leads to errors.

3. A lot of edits are necessary to use the code to compute a different integral these edits are likely to introduce errors.

The potential errors involved in point 2 serve to illustrate how important it is to use Matlab functions as mathematical functions. Here we have chosen to use the anonymous function to define the integrand as the variable v:

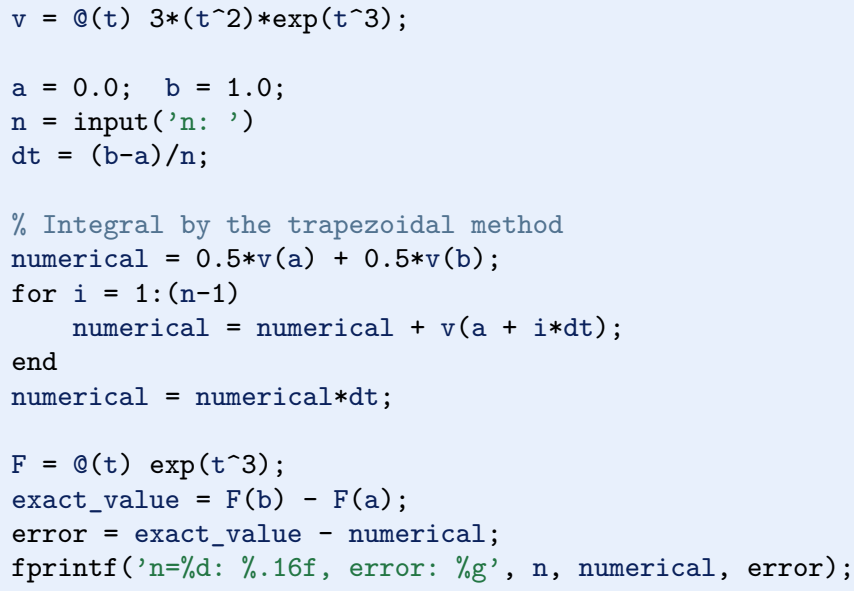

Unfortunately, the two other problems remain and they are fundamental.

Suppose you want to compute another integral, say $\int_{-1}^{1.1} e^{-x^{2}} d x$. How much do we need to change in the previous code to compute the new integral? Not so much:

- the formula for $\mathrm{v}$ must be replaced by a new formula

- the limits $\mathrm{a}$ and $\mathrm{b}$ 
- the anti-derivative $V$ is not easily known ${ }^{2}$ and can be omitted, and therefore we cannot write out the error

- the notation should be changed to be aligned with the new problem, i.e., $t$ and $d t$ changed to $x$ and $h$

These changes are straightforward to implement, but they are scattered around in the program, a fact that requires us to be very careful so we do not introduce new programming errors while we modify the code. It is also very easy to forget to make a required change.

With the previous code in trapezoidal.m, we can compute the new integral $\int_{-1}^{1.1} e^{-x^{2}} d x$ without touching the mathematical algorithm. In an interactive session (or in a program) we can just do

octave: $1>$ trapezoidal (@(x) $\left.\exp \left(-\mathrm{x}^{\wedge} 2\right),-1,1.1,400\right)$

ans $=1.5269$

When you now look back at the two solutions, the flat special-purpose program and the function-based program with the general-purpose function trapezoidal, you hopefully realize that implementing a general mathematical algorithm in a general function requires somewhat more abstract thinking, but the resulting code can be used over and over again. Essentially, if you apply the flat special-purpose style, you have to retest the implementation of the algorithm after every change of the program.

The present integral problems result in short code. In more challenging engineering problems the code quickly grows to hundreds and thousands of lines. Without abstractions in terms of general algorithms in general reusable functions, the complexity of the program grows so fast that it will be extremely difficult to make sure that the program works properly.

Another advantage of packaging mathematical algorithms in functions is that a function can be reused by anyone to solve a problem by just calling the function with a proper set of arguments. Understanding the function's inner details is not necessary to compute a new integral. Similarly, you can find libraries of functions on the Internet and use these functions to solve your problems without specific knowledge of every mathematical detail in the functions.

This desirable feature has its downside, of course: the user of a function may misuse it, and the function may contain programming errors and lead to wrong answers. Testing the output of downloaded functions is therefore extremely important before relying on the results.

\footnotetext{
${ }^{2}$ You cannot integrate $e^{-x^{2}}$ by hand, but this particular integral is appearing so often in so many contexts that the integral is a special function, called the Error function (http://en.wikipedia.org/ wiki/Error_function) and written $\operatorname{erf}(x)$. In a code, you can call erf $(x)$.
} 


\subsection{The Composite Midpoint Method}

The idea Rather than approximating the area under a curve by trapezoids, we can use plain rectangles. It may sound less accurate to use horizontal lines and not skew lines following the function to be integrated, but an integration method based on rectangles (the midpoint method) is in fact slightly more accurate than the one based on trapezoids!

In the midpoint method, we construct a rectangle for every sub-interval where the height equals $f$ at the midpoint of the sub-interval. Let us do this for four rectangles (Fig. 3.3), using the same sub-intervals as we had for hand calculations with the trapezoidal method: $[0,0.2),[0.2,0.6),[0.6,0.8)$, and $[0.8,1.0]$. We get

$$
\begin{aligned}
\int_{0}^{1} f(t) d t \approx & h_{1} f\left(\frac{0+0.2}{2}\right)+h_{2} f\left(\frac{0.2+0.6}{2}\right) \\
& +h_{3} f\left(\frac{0.6+0.8}{2}\right)+h_{4} f\left(\frac{0.8+1.0}{2}\right),
\end{aligned}
$$

where $h_{1}, h_{2}, h_{3}$, and $h_{4}$ are the widths of the sub-intervals, used previously with the trapezoidal method and defined in (3.10)-(3.13).

With $f(t)=3 t^{2} e^{t^{3}}$, the approximation becomes 1.632. Compared with the true answer (1.718), this is about $5 \%$ too small, but it is better than what we got with the trapezoidal method $(10 \%)$ with the same sub-intervals. More rectangles give a better approximation.

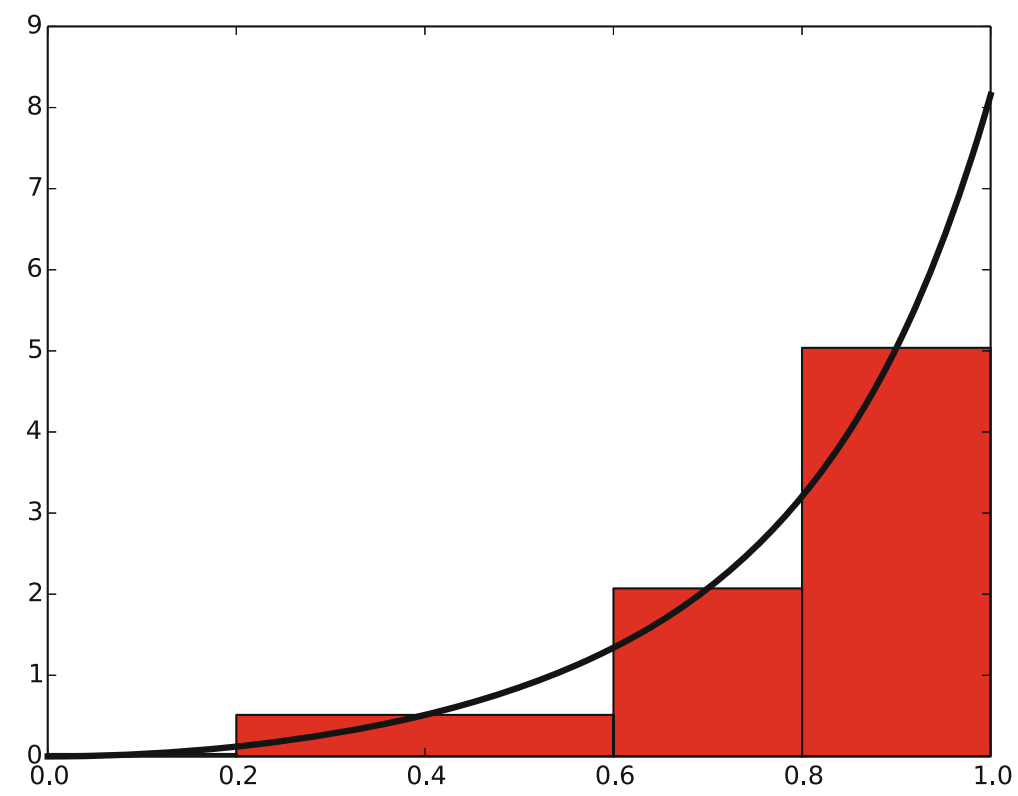

Fig. 3.3 Computing approximately the integral of a function as the sum of the areas of the rectangles 


\subsubsection{The General Formula}

Let us derive a formula for the midpoint method based on $n$ rectangles of equal width:

$$
\begin{aligned}
\int_{a}^{b} f(x) d x & =\int_{x_{0}}^{x_{1}} f(x) d x+\int_{x_{1}}^{x_{2}} f(x) d x+\ldots+\int_{x_{n-1}}^{x_{n}} f(x) d x \\
& \approx h f\left(\frac{x_{0}+x_{1}}{2}\right)+h f\left(\frac{x_{1}+x_{2}}{2}\right)+\ldots+h f\left(\frac{x_{n-1}+x_{n}}{2}\right) \\
& \approx h\left(f\left(\frac{x_{0}+x_{1}}{2}\right)+f\left(\frac{x_{1}+x_{2}}{2}\right)+\ldots+f\left(\frac{x_{n-1}+x_{n}}{2}\right)\right) .
\end{aligned}
$$

This sum may be written more compactly as

$$
\int_{a}^{b} f(x) d x \approx h \sum_{i=0}^{n-1} f\left(x_{i}\right)
$$

where $x_{i}=\left(a+\frac{h}{2}\right)+i h$.

\subsubsection{Implementation}

We follow the advice and lessons learned from the implementation of the trapezoidal method and make a function midpoint ( $f, a, b, n$ ) (in a file midpoint.m) for implementing the general formula (3.21):

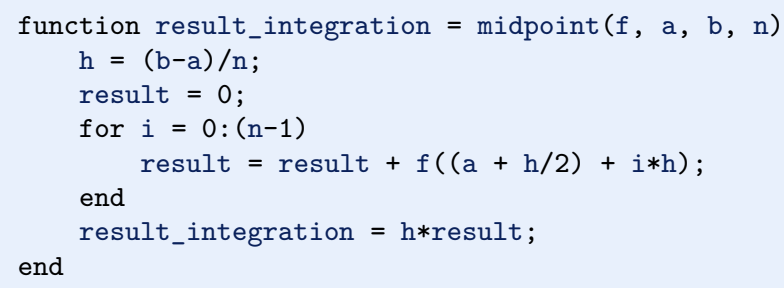

We can test the function as we explained for the similar trapezoidal method. The error in our particular problem $\int_{0}^{1} 3 t^{2} e^{t^{3}} d t$ with four intervals is now about 0.1 in contrast to 0.2 for the trapezoidal rule. This is in fact not accidental: one can show mathematically that the error of the midpoint method is a bit smaller than for the trapezoidal method. The differences are seldom of any practical importance, and on a laptop we can easily use $n=10^{6}$ and get the answer with an error of about $10^{-12}$ in a couple of seconds. 


\subsubsection{Comparing the Trapezoidal and the Midpoint Methods}

The next example shows how easy we can combine the trapezoidal and midpoint functions to make a comparison of the two methods in the file compare integration_methods.m:

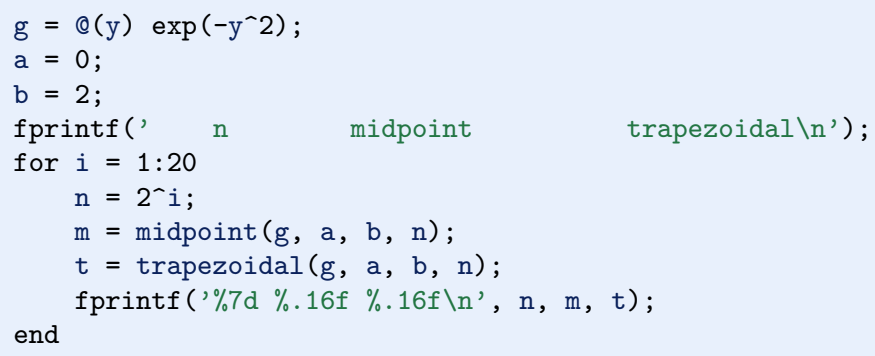

Note the efforts put into nice formatting - the output becomes

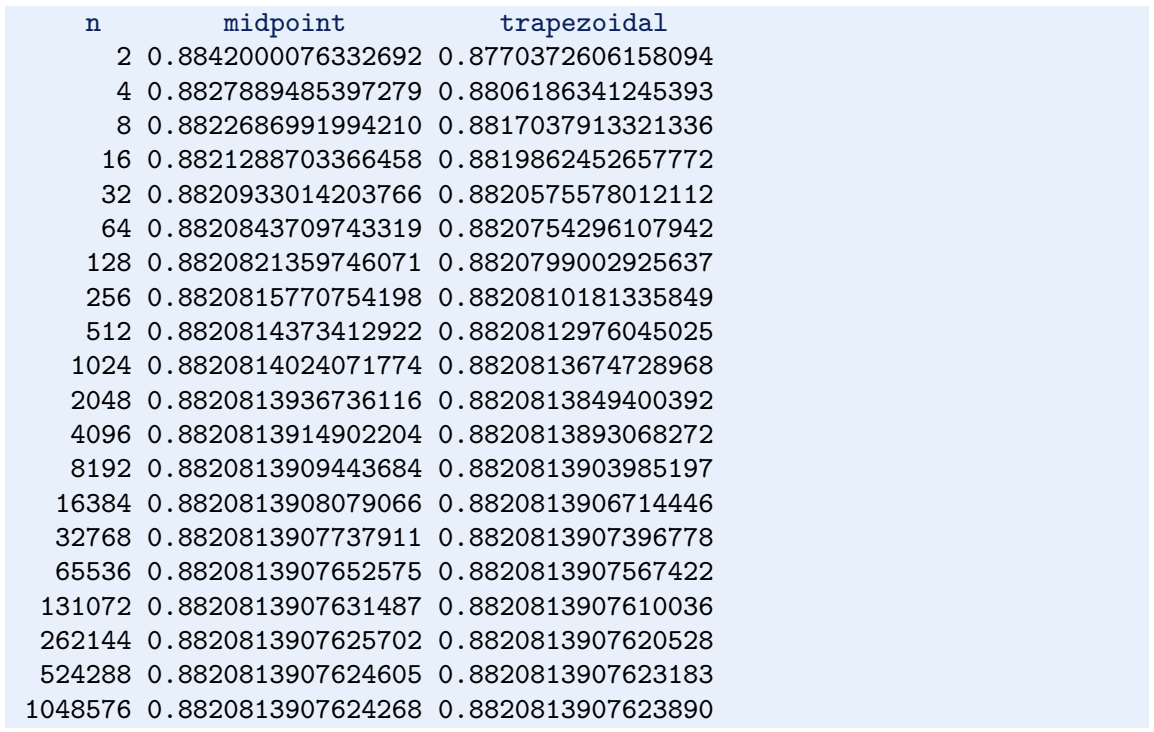

A visual inspection of the numbers shows how fast the digits stabilize in both methods. It appears that 13 digits have stabilized in the last two rows.

\section{Remark}

The trapezoidal and midpoint methods are just two examples in a jungle of numerical integration rules. Other famous methods are Simpson's rule and Gauss quadrature. They all work in the same way:

$$
\int_{a}^{b} f(x) d x \approx \sum_{i=0}^{n-1} w_{i} f\left(x_{i}\right)
$$


That is, the integral is approximated by a sum of function evaluations, where each evaluation $f\left(x_{i}\right)$ is given a weight $w_{i}$. The different methods differ in the way they construct the evaluation points $x_{i}$ and the weights $w_{i}$. We have used equally spaced points $x_{i}$, but higher accuracy can be obtained by optimizing the location of $x_{i}$.

\subsection{Testing}

\subsubsection{Problems with Brief Testing Procedures}

Testing of the programs for numerical integration has so far employed two strategies. If we have an exact answer, we compute the error and see that increasing $n$ decreases the error. When the exact answer is not available, we can (as in the comparison example in the previous section) look at the integral values and see that they stabilize as $n$ grows. Unfortunately, these are very weak test procedures and not at all satisfactory for claiming that the software we have produced is correctly implemented.

To see this, we can introduce a bug in the application function that calls trapezoidal: instead of integrating $3 t^{2} e^{t^{3}}$, we write "accidentally" $3 t^{3} e^{t^{3}}$, but keep the same anti-derivative $V(t) e^{t^{3}}$ for computing the error. With the bug and $n=4$, the error is 0.1 , but without the bug the error is 0.2 ! It is of course completely impossible to tell if 0.1 is the right value of the error. Fortunately, increasing $n$ shows that the error stays about 0.3 in the program with the bug, so the test procedure with increasing $n$ and checking that the error decreases points to a problem in the code.

Let us look at another bug, this time in the mathematical algorithm: instead of computing $\frac{1}{2}(f(a)+f(b))$ as we should, we forget the second $\frac{1}{2}$ and write $0.5 * \mathrm{f}(\mathrm{a})+\mathrm{f}(\mathrm{b})$. The error for $n=4,40,400$ when computing $\int_{1.1}^{1.9} 3 t^{2} e^{t^{3}} d t$ goes like 1400,107, 10, respectively, which looks promising. The problem is that the right errors should be $369,4.08$, and 0.04 . That is, the error should be reduced faster in the correct than in the buggy code. The problem, however, is that it is reduced in both codes, and we may stop further testing and believe everything is correctly implemented.

\section{Unit testing}

A good habit is to test small pieces of a larger code individually, one at a time. This is known as unit testing. One identifies a (small) unit of the code, and then one makes a separate test for this unit. The unit test should be stand-alone in the sense that it can be run without the outcome of other tests. Typically, one algorithm in scientific programs is considered as a unit. The challenge with unit tests in numerical computing is to deal with numerical approximation errors. A fortunate side effect of unit testing is that the programmer is forced to use functions to modularize the code into smaller, logical pieces. 


\subsubsection{Proper Test Procedures}

There are three serious ways to test the implementation of numerical methods via unit tests:

1. Comparing with hand-computed results in a problem with few arithmetic operations, i.e., small $n$.

2. Solving a problem without numerical errors. We know that the trapezoidal rule must be exact for linear functions. The error produced by the program must then be zero (to machine precision).

3. Demonstrating correct convergence rates. A strong test when we can compute exact errors, is to see how fast the error goes to zero as $n$ grows. In the trapezoidal and midpoint rules it is known that the error depends on $n$ as $n^{-2}$ as $n \rightarrow \infty$.

Hand-computed results Let us use two trapezoids and compute the integral $\int_{0}^{1} v(t), v(t)=3 t^{2} e^{t^{3}}:$

$$
\frac{1}{2} h(v(0)+v(0.5))+\frac{1}{2} h(v(0.5)+v(1))=2.463642041244344,
$$

when $h=0.5$ is the width of the two trapezoids. Running the program gives exactly the same result.

Solving a problem without numerical errors The best unit tests for numerical algorithms involve mathematical problems where we know the numerical result beforehand. Usually, numerical results contain unknown approximation errors, so knowing the numerical result implies that we have a problem where the approximation errors vanish. This feature may be present in very simple mathematical problems. For example, the trapezoidal method is exact for integration of linear functions $f(x)=a x+b$. We can therefore pick some linear function and construct a test function that checks equality between the exact analytical expression for the integral and the number computed by the implementation of the trapezoidal method.

A specific test case can be $\int_{1.2}^{4.4}(6 x-4) d x$. This integral involves an "arbitrary" interval $[1.2,4.4]$ and an "arbitrary" linear function $f(x)=6 x-4$. By "arbitrary" we mean expressions where we avoid the special numbers 0 and 1 since these have special properties in arithmetic operations (e.g., forgetting to multiply is equivalent to multiplying by 1 , and forgetting to add is equivalent to adding 0 ).

Demonstrating correct convergence rates Normally, unit tests must be based on problems where the numerical approximation errors in our implementation remain unknown. However, we often know or may assume a certain asymptotic behavior of the error. We can do some experimental runs with the test problem $\int_{0}^{1} 3 t^{2} e^{t^{3}} d t$ where $n$ is doubled in each run: $n=4,8,16$. The corresponding errors are then $12 \%, 3 \%$ and $0.77 \%$, respectively. These numbers indicate that the error is roughly reduced by a factor of 4 when doubling $n$. Thus, the error converges to zero as $n^{-2}$ and we say that the convergence rate is 2 . In fact, this result can also be shown 
mathematically for the trapezoidal and midpoint method. Numerical integration methods usually have an error that converge to zero as $n^{-p}$ for some $p$ that depends on the method. With such a result, it does not matter if we do not know what the actual approximation error is: we know at what rate it is reduced, so running the implementation for two or more different $n$ values will put us in a position to measure the expected rate and see if it is achieved.

The idea of a corresponding unit test is then to run the algorithm for some $n$ values, compute the error (the absolute value of the difference between the exact analytical result and the one produced by the numerical method), and check that the error has approximately correct asymptotic behavior, i.e., that the error is proportional to $n^{-2}$ in case of the trapezoidal and midpoint method.

Let us develop a more precise method for such unit tests based on convergence rates. We assume that the error $E$ depends on $n$ according to

$$
E=C n^{r}
$$

where $C$ is an unknown constant and $r$ is the convergence rate. Consider a set of experiments with various $n: n_{1}, n_{2}, \ldots, n_{q}$. We compute the corresponding errors $E_{1}, \ldots, E_{q}$. For two consecutive experiments, number $i$ and $i-1$, we have the error model

$$
\begin{aligned}
E_{i} & =C n_{i}^{r}, \\
E_{i-1} & =C n_{i-1}^{r} .
\end{aligned}
$$

These are two equations for two unknowns $C$ and $r$. We can easily eliminate $C$ by dividing the equations by each other. Then solving for $r$ gives

$$
r_{i}=\frac{\ln \left(E_{i} / E_{i-1}\right)}{\ln \left(n_{i} / n_{i-1}\right)} .
$$

We have introduced a subscript $i$ in $r$ since the estimated value for $r$ varies with $i$. Hopefully, $r_{i}$ approaches the correct convergence rate as the number of intervals increases and $i \rightarrow q$.

\subsubsection{Finite Precision of Floating-Point Numbers}

The test procedures above lead to comparison of numbers for checking that calculations were correct. Such comparison is more complicated than what a newcomer might think. Suppose we have a calculation $\mathrm{a}+\mathrm{b}$ and want to check that the result is what we expect. We start with $1+2$ :

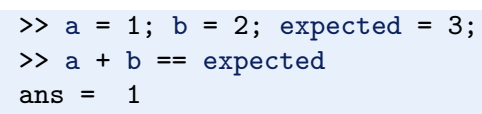


Then we proceed with $0.1+0.2$ :

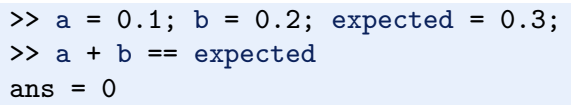

So why is $0.1+0.2 \neq 0.3$ ? The reason is that real numbers cannot in general be exactly represented on a computer. They must instead be approximated by a floating-point number ${ }^{3}$ that can only store a finite amount of information, usually about 17 digits of a real number. Let us print $0.1,0.2,0.1+0.2$, and 0.3 with 17 decimals:

> fprintf ( $\left.\% .17 f \backslash n \% .17 f \backslash n \% .17 f \backslash n \% .17 f \backslash n^{\prime}, 0.1,0.2,0.1+0.2,0.3\right)$

0.10000000000000001

0.20000000000000001

0.30000000000000004

0.29999999999999999

We see that all of the numbers have an inaccurate digit in the 17 th decimal place. Because $0.1+0.2$ evaluates to 0.30000000000000004 and 0.3 is represented as 0.29999999999999999 , these two numbers are not equal. In general, real numbers in Matlab have (at most) 16 correct decimals.

When we compute with real numbers, these numbers are inaccurately represented on the computer, and arithmetic operations with inaccurate numbers lead to small rounding errors in the final results. Depending on the type of numerical algorithm, the rounding errors may or may not accumulate.

If we cannot make tests like $0.1+0.2=0.3$, what should we then do? The answer is that we must accept some small inaccuracy and make a test with a tolerance. Here is the recipe:

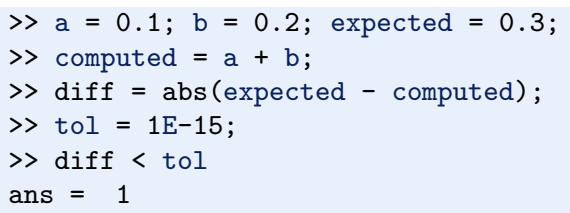

Here we have set the tolerance for comparison to $10^{-15}$, but calculating $0.3-$ $(0.1+0.2)$ shows that it equals $-5.55 e-17$, so a lower tolerance could be used in this particular example. However, in other calculations we have little idea about how accurate the answer is (there could be accumulation of rounding errors in more complicated algorithms), so $10^{-15}$ or $10^{-14}$ are robust values. As we demonstrate below, these tolerances depend on the magnitude of the numbers in the calculations.

Doing an experiment with $10^{k}+0.3-\left(10^{k}+0.1+0.2\right)$ for $k=1, \ldots, 10$ shows that the answer (which should be zero) is around $10^{16-k}$. This means that the tolerance must be larger if we compute with larger numbers. Setting a proper tolerance therefore requires some experiments to see what level of accuracy one can expect. A way out of this difficulty is to work with relative instead of absolute

${ }^{3}$ https://en.wikipedia.org/wiki/Floating_point 
differences. In a relative difference we divide by one of the operands, e.g.,

$$
a=10^{k}+0.3, \quad b=\left(10^{k}+0.1+0.2\right), \quad c=\frac{a-b}{a} .
$$

Computing this $c$ for various $k$ shows a value around $10^{-16}$. A safer procedure is thus to use relative differences.

\subsubsection{Constructing Unit Tests and Writing Test Functions}

Software testing in other languages often applies comprehensive test frameworks to automatically run through large numbers of tests. This is very advantageous as one can at any time check that the code works. It is a good habit to run the test suite after every edit of the source code files.

Matlab also has test frameworks, but we shall here just use the common ideas (across languages) for writing tests and not employ any particular framework for running the tests. Our convention is to put each test in a separate test function, with the following properties:

- the name must start with test_

- the test function cannot have any arguments

- the tests inside test functions must be boolean expressions

- a boolean expression $b$ must be tested with assert (b, msg), where msg is an optional object (string or number) to be written out when $\mathrm{b}$ is false

Suppose we have written a function

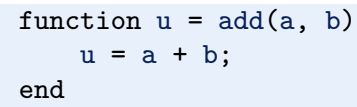

A corresponding test function might then be

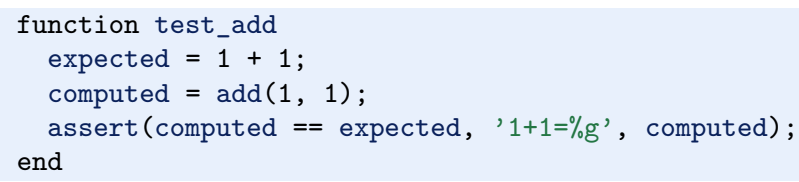

Test functions and their calls are conveniently placed in files whose names start with test_. A simple script can be made to search for such files and run them automatically (essentially, this is what testing frameworks do).

As long as we add integers, the equality test in the test_add function is appropriate, but if we try to call add $(0.1,0.2)$ instead, we will face the rounding error problems explained in Sect. 3.4.3, and we must use a test with tolerance instead: 


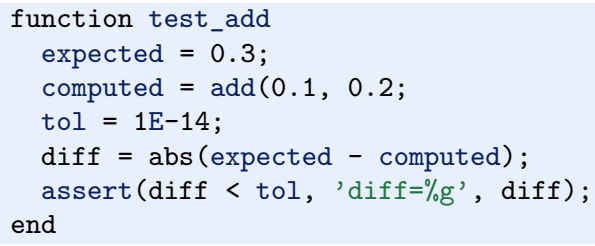

Below we shall write test functions for each of the three test procedures we suggested: comparison with hand calculations, checking problems that can be exactly solved, and checking convergence rates. We stick to testing the trapezoidal integration code and collect all test functions in one common file by the name test_trapezoidal.m.

In Matlab, we need to enforce the following rules on files for the function to be tested and the test functions:

- The numerical method (to be tested) must be available as a function in a file with the same name as the function.

- The test functions are put in separate files.

Hand-computed numerical results Our previous hand calculations for two trapezoids can be checked against the trapezoidal function inside a test function (in a file test_trapezoidal.m):

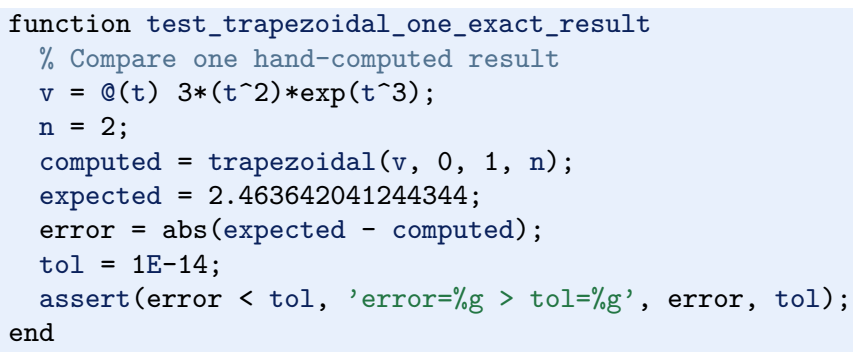

Note the importance of checking err against exact with a tolerance: rounding errors from the arithmetics inside trapezoidal will not make the result exactly like the hand-computed one. The size of the tolerance is here set to $10^{-14}$, which is a kind of all-round value for computations with numbers not deviating much from unity.

Solving a problem without numerical errors We know that the trapezoidal rule is exact for linear integrands. Choosing the integral $\int_{1.2}^{4.4}(6 x-4) d x$ as test case, the corresponding test function for this unit test may look like

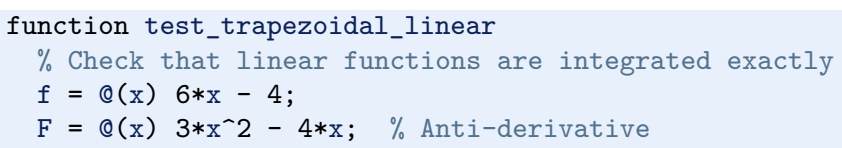




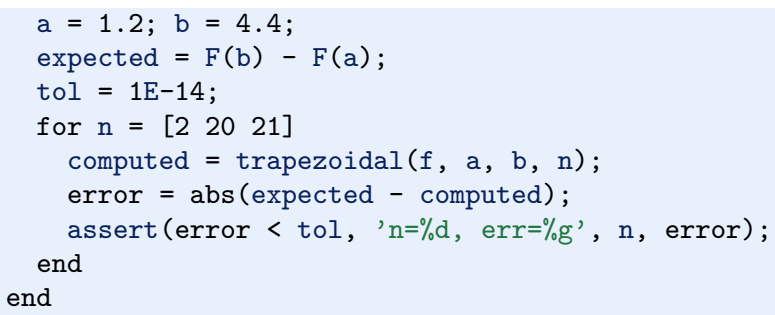

Demonstrating correct convergence rates In the present example with integration, it is known that the approximation errors in the trapezoidal rule are proportional to $n^{-2}, n$ being the number of subintervals used in the composite rule.

Computing convergence rates requires somewhat more tedious programming than the previous tests, but can be applied to more general integrands. The algorithm typically goes like

- for $i=1,2, \ldots, q$

- $n_{i}=2^{i}$

- Compute integral with $n_{i}$ intervals

- Compute the error $E_{i}$

- Estimate $r_{i}$ from (3.24) if $i>1$

The corresponding code may look like

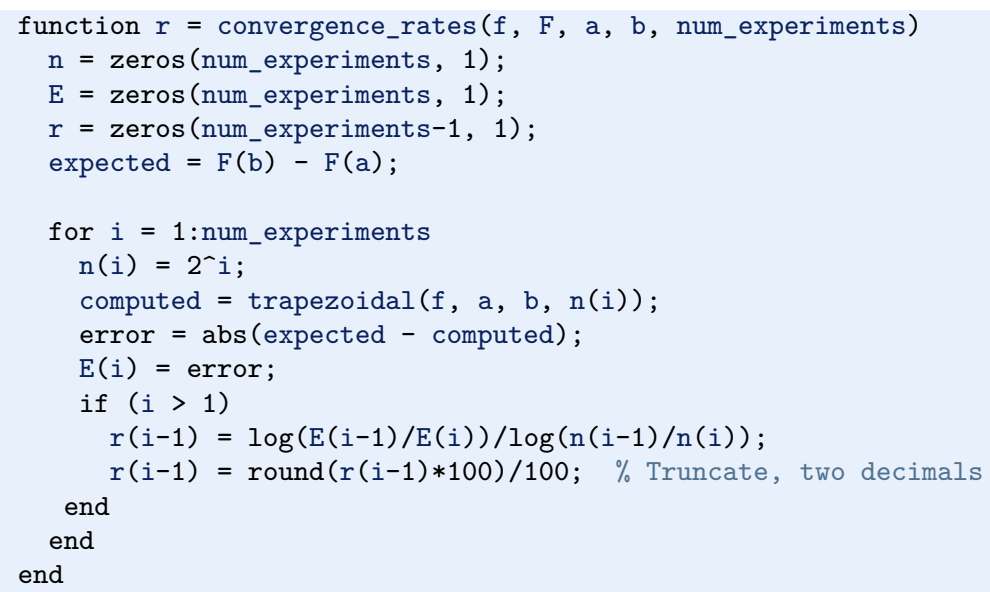

Making a test function is a matter of choosing $f, F, a$, and $b$, and then checking the value of $r_{i}$ for the largest $i$ :

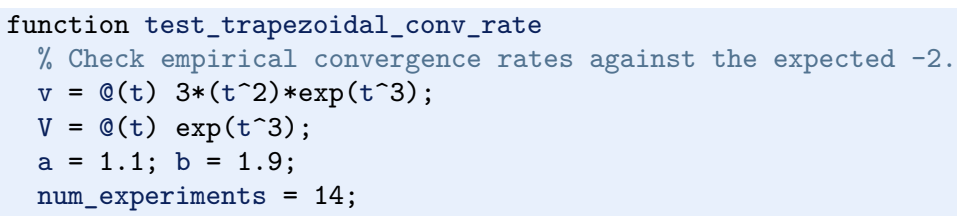




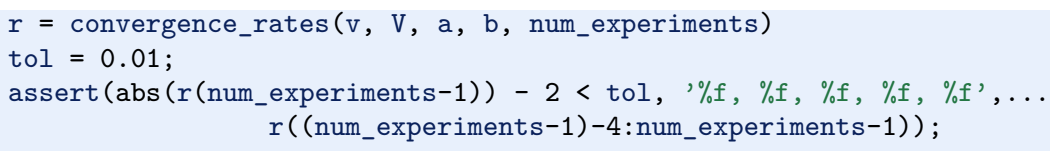

Running the test shows that all $r_{i}$, except the first one, equal the target limit 2 within two decimals. This observation suggests a tolerance of $10^{-2}$.

\section{Remark about version control of files}

Having a suite of test functions for automatically checking that your software works is considered as a fundamental requirement for reliable computing. Equally important is a system that can keep track of different versions of the files and the tests, known as a version control system. Today's most popular version control system is $\mathrm{Git}^{4}$, which the authors strongly recommend the reader to use for programming and writing reports. The combination of Git and cloud storage such as GitHub is a very common way of organizing scientific or engineering work. We have a quick intro ${ }^{5}$ to Git and GitHub that gets you up and running within minutes.

The typical workflow with Git goes as follows.

1. Before you start working with files, make sure you have the latest version of them by running git pull.

2. Edit files, remove or create files (new files must be registered by git add).

3. When a natural piece of work is done, commit your changes by the git commit command.

4. Implement your changes also in the cloud by doing git push.

A nice feature of Git is that people can edit the same file at the same time and very often Git will be able to automatically merge the changes (!). Therefore, version control is crucial when you work with others or when you do your work on different types of computers. Another key feature is that anyone can at any time view the history of a file, see who did what when, and roll back the entire file collection to a previous commit. This feature is, of course, fundamental for reliable work.

\subsection{Vectorization}

The functions midpoint and trapezoid usually run fast in Matlab and compute an integral to a satisfactory precision within a fraction of a second. However, long loops in Matlab may run slowly in more complicated implementations. To increase the speed, the loops can be replaced by vectorized code. The integration functions constitute a simple and good example to illustrate how to vectorize loops.

We have already seen simple examples on vectorization in Sect. 1.4 when we could evaluate a mathematical function $f(x)$ for a large number of $x$ values stored in an array. Basically, we can write

\footnotetext{
${ }^{4}$ https://en.wikipedia.org/wiki/Git_(software)

${ }^{5}$ http://hplgit.github.io/teamods/bitgit/Langtangen_bitgit-bootstrap.html
} 


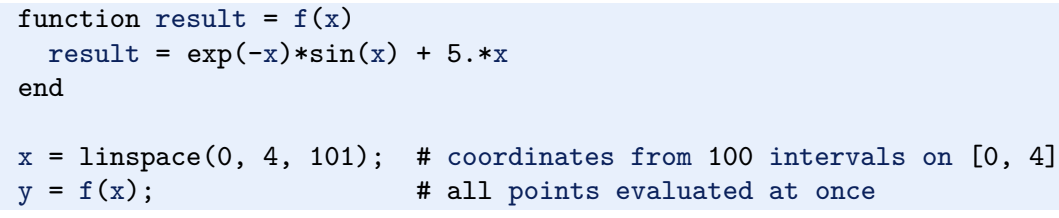

The result $y$ is the array that would be computed if we ran a for loop over the individual $x$ values and called $f$ for each value. Vectorization essentially eliminates this loop in Matlab (i.e., the looping over $x$ and application of $f$ to each $x$ value are instead performed in a library with fast, compiled code).

Vectorizing the midpoint rule The aim of vectorizing the midpoint and trapezoidal functions is also to remove the explicit loop in Matlab. We start with vectorizing the midpoint function since trapezoid is not equally straightforward. The fundamental ideas of the vectorized algorithm are to

1. compute all the evaluation points in one array $x$

2. call $f(x)$ to produce an array of corresponding function values

3. use the sum function to sum the $f(x)$ values

The evaluation points in the midpoint method are $x_{i}=a+\left(i+\frac{1}{2}\right) h, i=0, \ldots, n-$ 1. That is, $n$ uniformly distributed coordinates between $a+h / 2$ and $b-h / 2$. Such coordinates can be calculated by $\mathrm{x}=\operatorname{linspace}(\mathrm{a}+\mathrm{h} / 2, \mathrm{~b}-\mathrm{h} / 2, \mathrm{n})$. Given that the Matlab implementation $f$ of the mathematical function $f$ works with an array argument, which requires array versions of arithmetic operators $(.+, . *$, etc.) in Matlab, $f(x)$ will produce all the function values in an array. The array elements are then summed up by sum: $\operatorname{sum}(f(x))$. This sum is to be multiplied by the rectangle width $h$ to produce the integral value. The complete function is listed below.

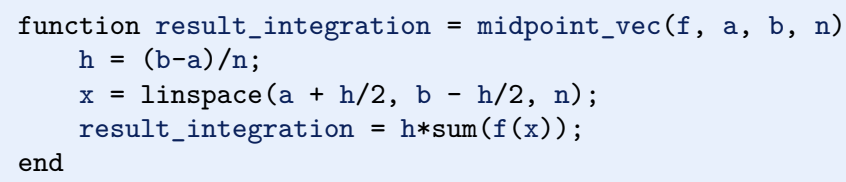

The code is found in the file midpoint_vec.m. An interactive test reads

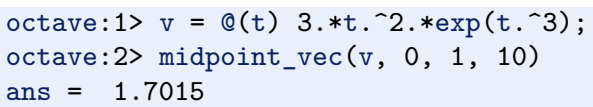

Note the need for the vectorized operator $* *$ in the function expression since $\mathrm{v}(\mathrm{x})$ will be called with array arguments $\mathrm{x}$.

The vectorized code performs all loops very efficiently in compiled code, resulting in much faster execution. Moreover, many readers of the code will also say that the algorithm looks clearer than in the loop-based implementation. 
Vectorizing the trapezoidal rule We can use the same approach to vectorize the trapezoid function. However, the trapezoidal rule performs a sum where the end points have different weight. If we do $\operatorname{sum}(f(x))$, we get the end points $f(a)$ and $f(b)$ with weight unity instead of one half. A remedy is to subtract the error from $\operatorname{sum}(f(x)): \operatorname{sum}(f(x))-0.5 * f(a)-0.5 * f(b)$. The vectorized version of the trapezoidal method then becomes (code in trapezoidal_vec.m)

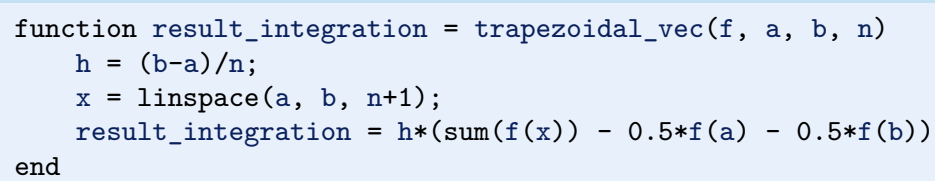

\subsection{Measuring Computational Speed}

Now that we have created faster, vectorized versions of functions in the previous section, it is interesting to measure how much faster they are. The purpose of the present section is therefore to explain how we can record the CPU time consumed by a function so we can answer this question. The "stop watch" in Matlab is the function pair tic (start) and toc. Here is an interactive session measuring the effect of midpoint_vec versus midpoint:

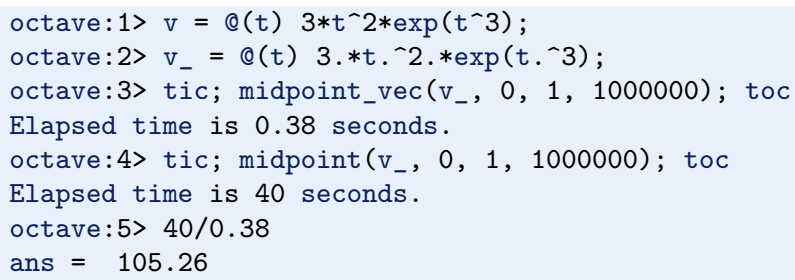

The vectorized version is 100 times faster!

\subsection{Double and Triple Integrals}

\subsubsection{The Midpoint Rule for a Double Integral}

Given a double integral over a rectangular domain $[a, b] \times[c, d]$,

$$
\int_{a}^{b} \int_{c}^{d} f(x, y) d y d x
$$

how can we approximate this integral by numerical methods?

Derivation via one-dimensional integrals Since we know how to deal with integrals in one variable, a fruitful approach is to view the double integral as two 
integrals, each in one variable, which can be approximated numerically by previous one-dimensional formulas. To this end, we introduce a help function $g(x)$ and write

$$
\int_{a}^{b} \int_{c}^{d} f(x, y) d y d x=\int_{a}^{b} g(x) d x, \quad g(x)=\int_{c}^{d} f(x, y) d y .
$$

Each of the integrals

$$
\int_{a}^{b} g(x) d x, \quad g(x)=\int_{c}^{d} f(x, y) d y
$$

can be discretized by any numerical integration rule for an integral in one variable. Let us use the midpoint method (3.21) and start with $g(x)=\int_{c}^{d} f(x, y) d y$. We introduce $n_{y}$ intervals on $[c, d]$ with length $h_{y}$. The midpoint rule for this integral then becomes

$$
g(x)=\int_{c}^{d} f(x, y) d y \approx h_{y} \sum_{j=0}^{n_{y}-1} f\left(x, y_{j}\right), \quad y_{j}=c+\frac{1}{2} h_{y}+j h_{y} .
$$

The expression looks somewhat different from (3.21), but that is because of the notation: since we integrate in the $y$ direction and will have to work with both $x$ and $y$ as coordinates, we must use $n_{y}$ for $n, h_{y}$ for $h$, and the counter $i$ is more naturally called $j$ when integrating in $y$. Integrals in the $x$ direction will use $h_{x}$ and $n_{x}$ for $h$ and $n$, and $i$ as counter.

The double integral is $\int_{a}^{b} g(x) d x$, which can be approximated by the midpoint method:

$$
\int_{a}^{b} g(x) d x \approx h_{x} \sum_{i=0}^{n_{x}-1} g\left(x_{i}\right), \quad x_{i}=a+\frac{1}{2} h_{x}+i h_{x} .
$$

Putting the formulas together, we arrive at the composite midpoint method for a double integral:

$$
\begin{aligned}
\int_{a}^{b} \int_{c}^{d} f(x, y) d y d x & \approx h_{x} \sum_{i=0}^{n_{x}-1} h_{y} \sum_{j=0}^{n_{y}-1} f\left(x_{i}, y_{j}\right) \\
& =h_{x} h_{y} \sum_{i=0}^{n_{x}-1} \sum_{j=0}^{n_{y}-1} f\left(a+\frac{h_{x}}{2}+i h_{x}, c+\frac{h_{y}}{2}+j h_{y}\right) .
\end{aligned}
$$

Direct derivation The formula (3.25) can also be derived directly in the twodimensional case by applying the idea of the midpoint method. We divide the rectangle $[a, b] \times[c, d]$ into $n_{x} \times n_{y}$ equal-sized cells. The idea of the midpoint method is to approximate $f$ by a constant over each cell, and evaluate the constant at the midpoint. Cell $(i, j)$ occupies the area

$$
\left[a+i h_{x}, a+(i+1) h_{x}\right] \times\left[c+j h_{y}, c+(j+1) h_{y}\right]
$$


and the midpoint is $\left(x_{i}, y_{j}\right)$ with

$$
x_{i}=a+i h_{x}+\frac{1}{2} h_{x}, \quad y_{j}=c+j h_{y}+\frac{1}{2} h_{y} .
$$

The integral over the cell is therefore $h_{x} h_{y} f\left(x_{i}, y_{j}\right)$, and the total double integral is the sum over all cells, which is nothing but formula (3.25).

Programming a double sum The formula (3.25) involves a double sum, which is normally implemented as a double for loop. A Matlab function implementing (3.25) may look like

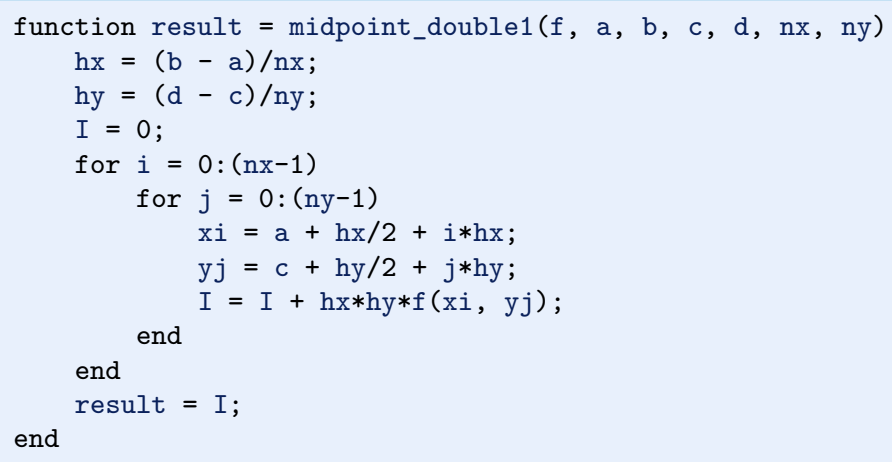

With this function, which is available in the file midpoint_double1.m, we may now compute some integral $\int_{0}^{2} \int_{2}^{3}(2 x+y) d y d x=9$ in an interactive shell and demonstrate that the function computes the right number:

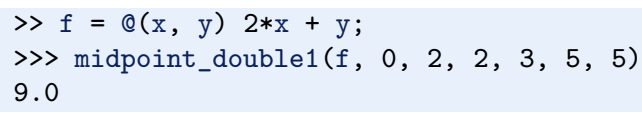

Reusing code for one-dimensional integrals It is very natural to write a twodimensional midpoint method as we did in function midpoint_double1 when we have the formula (3.25). However, we could alternatively ask, much as we did in the mathematics, can we reuse a well-tested implementation for one-dimensional integrals to compute double integrals? That is, can we use function midpoint

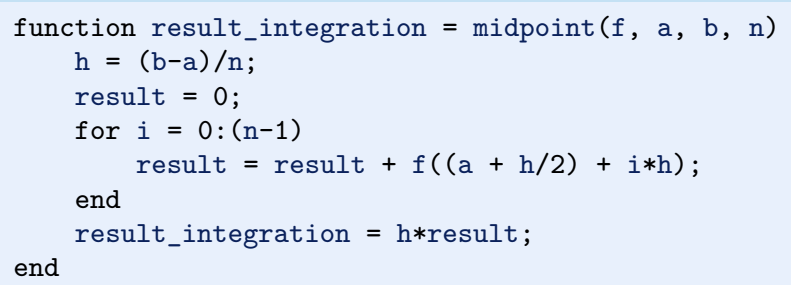

from Sect. 3.3.2 "twice"? The answer is yes, if we think as we did in the mathematics: compute the double integral as a midpoint rule for integrating $g(x)$ and define 
$g\left(x_{i}\right)$ in terms of a midpoint rule over $f$ in the $y$ coordinate. The corresponding function has very short code:

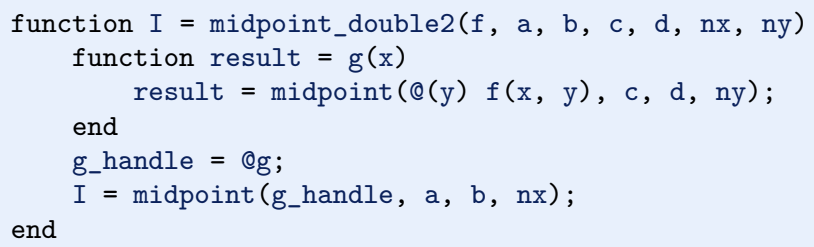

The important advantage of this implementation is that we reuse a well-tested function for the standard one-dimensional midpoint rule and that we apply the onedimensional rule exactly as in the mathematics.

Verification via test functions How can we test that our functions for the double integral work? The best unit test is to find a problem where the numerical approximation error vanishes because then we know exactly what the numerical answer should be. The midpoint rule is exact for linear functions, regardless of how many subinterval we use. Also, any linear two-dimensional function $f(x, y)=$ $p x+q y+r$ will be integrated exactly by the two-dimensional midpoint rule. We may pick $f(x, y)=2 x+y$ and create a proper test function that can automatically verify our two alternative implementations of the two-dimensional midpoint rule. To compute the integral of $f(x, y)$ we take advantage of SymPy to eliminate the possibility of errors in hand calculations. The test function becomes

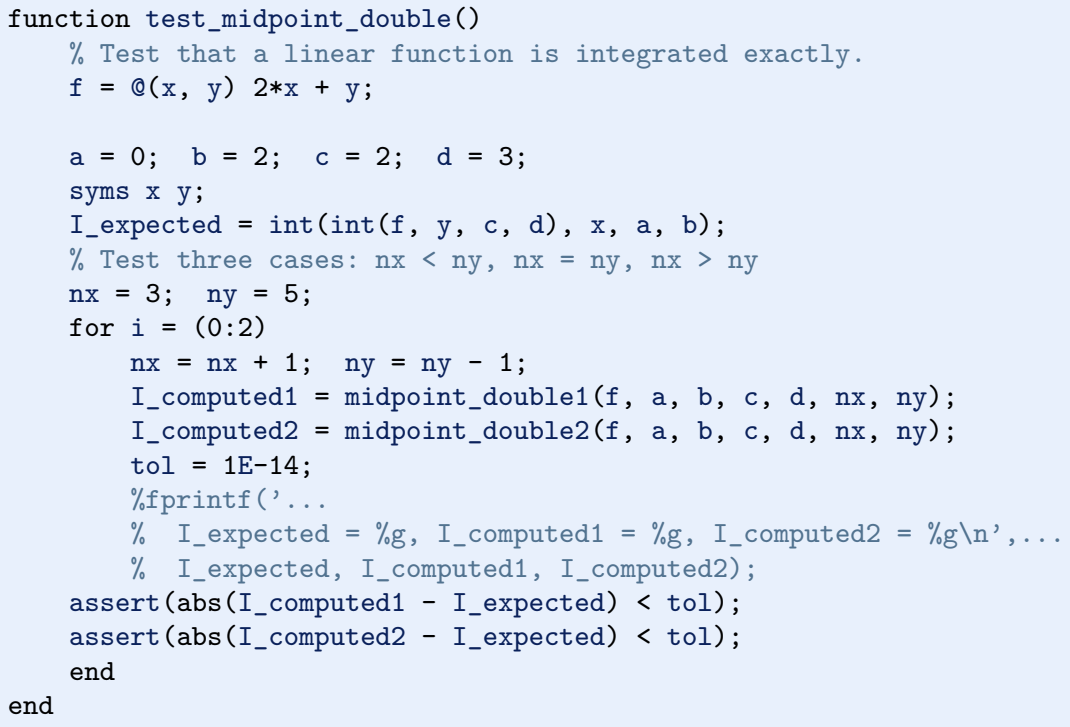




\section{Let test functions speak up?}

If we call the above test_midpoint_double function and nothing happens, our implementations are correct. However, it is somewhat annoying to have a function that is completely silent when it works - are we sure all things are properly computed? During development it is therefore highly recommended to insert a print statement such that we can monitor the calculations and be convinced that the test function does what we want. Since a test function should not have any print statement, we simply comment it out as we have done in the function listed above.

The trapezoidal method can be used as alternative for the midpoint method. The derivation of a formula for the double integral and the implementations follow exactly the same ideas as we explained with the midpoint method, but there are more terms to write in the formulas. Exercise 3.13 asks you to carry out the details. That exercise is a very good test on your understanding of the mathematical and programming ideas in the present section.

\subsubsection{The Midpoint Rule for a Triple Integral}

Theory Once a method that works for a one-dimensional problem is generalized to two dimensions, it is usually quite straightforward to extend the method to three dimensions. This will now be demonstrated for integrals. We have the triple integral

$$
\int_{a}^{b} \int_{c}^{d} \int_{e}^{f} g(x, y, z) d z d y d x
$$

and want to approximate the integral by a midpoint rule. Following the ideas for the double integral, we split this integral into one-dimensional integrals:

$$
\begin{aligned}
p(x, y) & =\int_{e}^{f} g(x, y, z) d z \\
q(x) & =\int_{c}^{d} p(x, y) d y \\
\int_{a}^{b} \int_{c}^{d} \int_{e}^{b} g(x, y, z) d z d y d x & =\int_{a}^{b} q(x) d x
\end{aligned}
$$


For each of these one-dimensional integrals we apply the midpoint rule:

$$
\begin{aligned}
p(x, y)=\int_{e}^{f} g(x, y, z) d z & \approx \sum_{k=0}^{n_{z}-1} g\left(x, y, z_{k}\right), \\
q(x)=\int_{c}^{d} p(x, y) d y & \approx \sum_{j=0}^{n_{y}-1} p\left(x, y_{j}\right), \\
\int_{a}^{b} \int_{c}^{d} \int_{e}^{f} g(x, y, z) d z d y d x=\int_{a}^{b} q(x) d x & \approx \sum_{i=0}^{n_{x}-1} q\left(x_{i}\right),
\end{aligned}
$$

where

$$
z_{k}=e+\frac{1}{2} h_{z}+k h_{z}, \quad y_{j}=c+\frac{1}{2} h_{y}+j h_{y} \quad x_{i}=a+\frac{1}{2} h_{x}+i h_{x} .
$$

Starting with the formula for $\int_{a}^{b} \int_{c}^{d} \int_{e}^{f} g(x, y, z) d z d y d x$ and inserting the two previous formulas gives

$$
\begin{aligned}
& \int_{a}^{b} \int_{c}^{d} \int_{e}^{f} g(x, y, z) d z d y d x \\
& \approx h_{x} h_{y} h_{z} \sum_{i=0}^{n_{x}-1} \sum_{j=0}^{n_{y}-1} \sum_{k=0}^{n_{z}-1} g\left(a+\frac{1}{2} h_{x}+i h_{x}, c+\frac{1}{2} h_{y}+j h_{y}, e+\frac{1}{2} h_{z}+k h_{z}\right) .
\end{aligned}
$$

Note that we may apply the ideas under Direct derivation at the end of Sect. 3.7.1 to arrive at (3.26) directly: divide the domain into $n_{x} \times n_{y} \times n_{z}$ cells of volumes $h_{x} h_{y} h_{z}$; approximate $g$ by a constant, evaluated at the midpoint $\left(x_{i}, y_{j}, z_{k}\right)$, in each cell; and sum the cell integrals $h_{x} h_{y} h_{z} g\left(x_{i}, y_{j}, z_{k}\right)$.

Implementation We follow the ideas for the implementations of the midpoint rule for a double integral. The corresponding functions are shown below and found in the files midpoint_triple1.m, midpoint.m, midpoint_triple2.m, test_midpoint_triple.m.

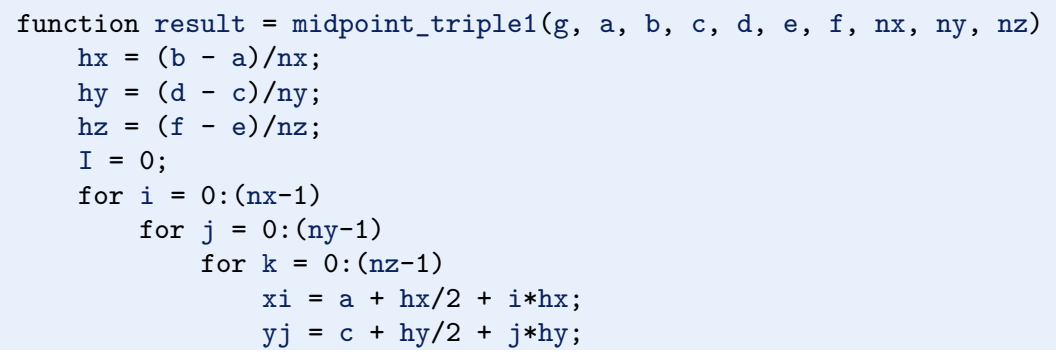




\subsubsection{Monte Carlo Integration for Complex-Shaped Domains}

Repeated use of one-dimensional integration rules to handle double and triple integrals constitute a working strategy only if the integration domain is a rectangle or box. For any other shape of domain, completely different methods must be used. A common approach for two- and three-dimensional domains is to divide the domain into many small triangles or tetrahedra and use numerical integration methods for each triangle or tetrahedron. The overall algorithm and implementation is too complicated to be addressed in this book. Instead, we shall employ an alternative, very simple and general method, called Monte Carlo integration. It can be implemented in half a page of code, but requires orders of magnitude more function evaluations in double integrals compared to the midpoint rule.

However, Monte Carlo integration is much more computationally efficient than the midpoint rule when computing higher-dimensional integrals in more than three variables over hypercube domains. Our ideas for double and triple integrals can easily be generalized to handle an integral in $m$ variables. A midpoint formula then involves $m$ sums. With $n$ cells in each coordinate direction, the formula requires $n^{m}$ function evaluations. That is, the computational work explodes as an exponential function of the number of space dimensions. Monte Carlo integration, on the other hand, does not suffer from this explosion of computational work and is the preferred method for computing higher-dimensional integrals. So, it makes sense in a chapter on numerical integration to address Monte Carlo methods, both for handling complex domains and for handling integrals with many variables.

The Monte Carlo integration algorithm The idea of Monte Carlo integration of $\int_{a}^{b} f(x) d x$ is to use the mean-value theorem from calculus, which states that the integral $\int_{a}^{b} f(x) d x$ equals the length of the integration domain, here $b-a$, times the average value of $f, \bar{f}$, in $[a, b]$. The average value can be computed by sampling $f$ at a set of random points inside the domain and take the mean of the function values. In higher dimensions, an integral is estimated as the area/volume of the domain times the average value, and again one can evaluate the integrand at a set of random points in the domain and compute the mean value of those evaluations.

Let us introduce some quantities to help us make the specification of the integration algorithm more precise. Suppose we have some two-dimensional integral

$$
\int_{\Omega} f(x, y) d x d x,
$$

where $\Omega$ is a two-dimensional domain defined via a help function $g(x, y)$ :

$$
\Omega=\{(x, y) \mid g(x, y) \geq 0\}
$$

That is, points $(x, y)$ for which $g(x, y) \geq 0$ lie inside $\Omega$, and points for which $g(x, y)<\Omega$ are outside $\Omega$. The boundary of the domain $\partial \Omega$ is given by the implicit curve $g(x, y)=0$. Such formulations of geometries have been very common during the last couple of decades, and one refers to $g$ as a level-set function and the boundary $g=0$ as the zero-level contour of the level-set function. For simple geometries one can easily construct $g$ by hand, while in more complicated industrial applications one must resort to mathematical models for constructing $g$. 
Let $A(\Omega)$ be the area of a domain $\Omega$. We can estimate the integral by this Monte Carlo integration method:

1. embed the geometry $\Omega$ in a rectangular area $R$

2. draw a large number of random points $(x, y)$ in $R$

3. count the fraction $q$ of points that are inside $\Omega$

4. approximate $A(\Omega) / A(R)$ by $q$, i.e., set $A(\Omega)=q A(R)$

5. evaluate the mean of $f, \bar{f}$, at the points inside $\Omega$

6. estimate the integral as $A(\Omega) \bar{f}$

Note that $A(R)$ is trivial to compute since $R$ is a rectangle, while $A(\Omega)$ is unknown. However, if we assume that the fraction of $A(R)$ occupied by $A(\Omega)$ is the same as the fraction of random points inside $\Omega$, we get a simple estimate for $A(\Omega)$.

To get an idea of the method, consider a circular domain $\Omega$ embedded in a rectangle as shown below. A collection of random points is illustrated by black dots.

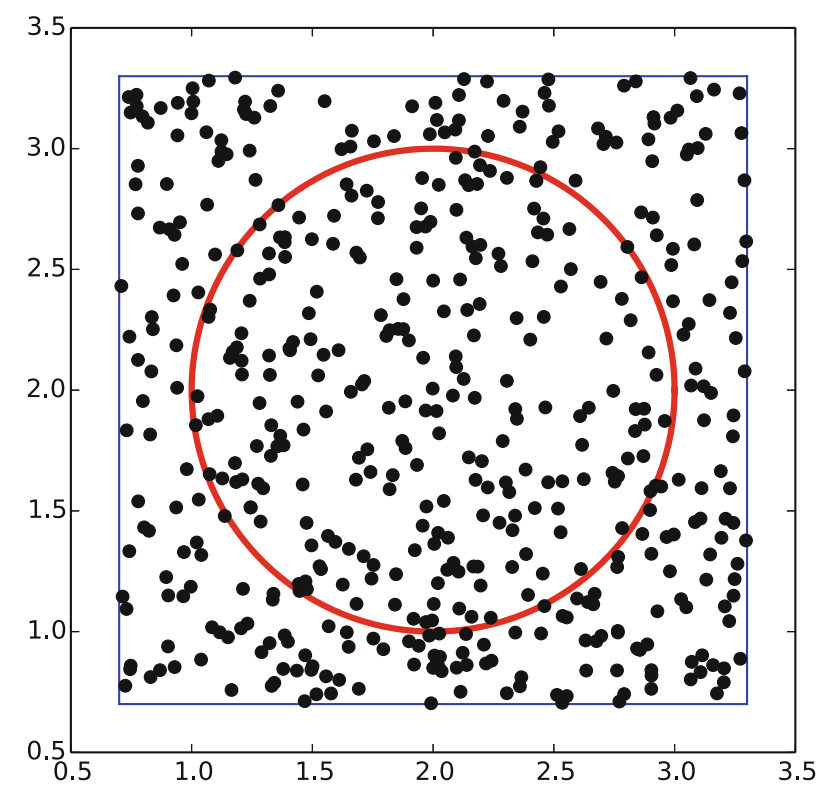

Implementation A Matlab function implementing $\int_{\Omega} f(x, y) d x d y$ can be written like this:

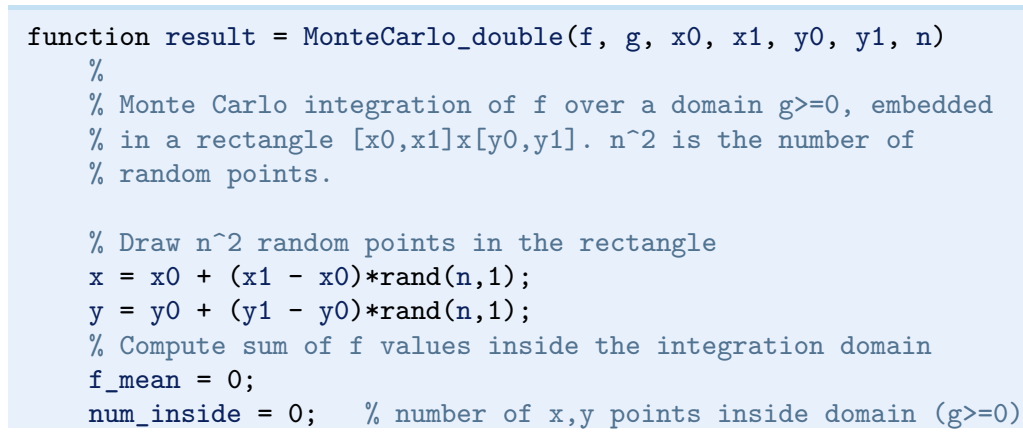




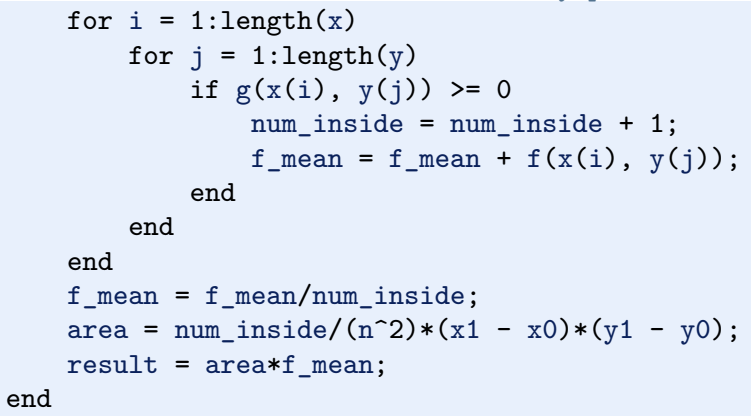

(See the file MonteCarlo_double.m.)

Verification A simple test case is to check the area of a rectangle $[0,2] \times[3,4.5]$ embedded in a rectangle $[0,3] \times[2,5]$. The right answer is 3 , but Monte Carlo integration is, unfortunately, never exact so it is impossible to predict the output of the algorithm. All we know is that the estimated integral should approach 3 as the number of random points goes to infinity. Also, for a fixed number of points, we can run the algorithm several times and get different numbers that fluctuate around the exact value, since different sample points are used in different calls to the Monte Carlo integration algorithm.

The area of the rectangle can be computed by the integral $\int_{0}^{2} \int_{3}^{4.5} d y d x$, so in this case we identify $f(x, y)=1$, and the $g$ function can be specified as (e.g.) 1 if $(x, y)$ is inside $[0,2] \times[3,4.5]$ and -1 otherwise. Here is an example on how we can utilize the MonteCarlo_double function to compute the area for different number of samples:

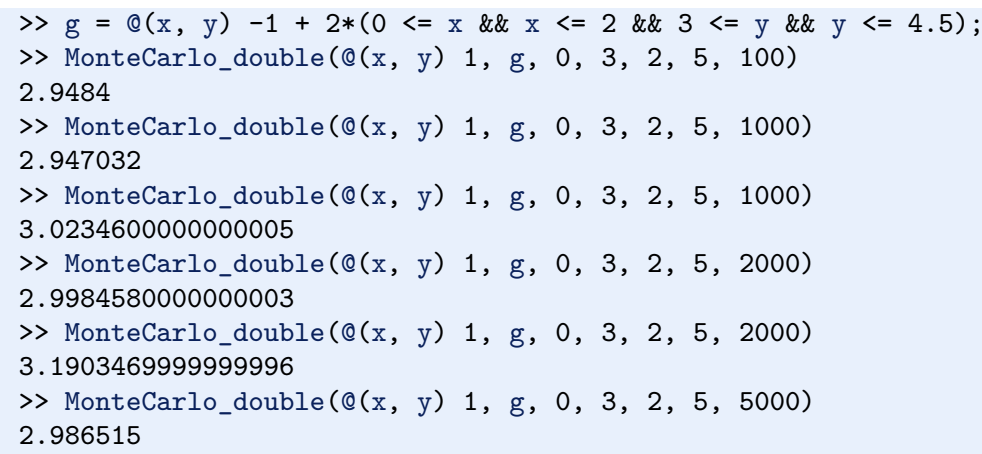

To get a one-line definition of g, we have exploited the fact that each of the boolean tests (in parenthesis separated by \&\&) will evaluate to either 0 (if false) or 1 (if true). If all of them evaluate to true, the whole parenthesis will evaluate to 1 and the number 1 (from $-1+2 * 1$ ) is returned. On the other hand, if any single one of the boolean tests evaluate to false, the parenthesis will evaluate to 0 and the number -1 (from $-1+2 * 0$ ) is returned. We see that the values fluctuate around 3 , a fact that supports a correct implementation, but in principle, bugs could be hidden behind the inaccurate answers. 
It is mathematically known that the standard deviation of the Monte Carlo estimate of an integral converges as $n^{-1 / 2}$, where $n$ is the number of samples. This kind of convergence rate estimate could be used to verify the implementation, but this topic is beyond the scope of this book.

Test function for function with random numbers To make a test function, we need a unit test that has identical behavior each time we run the test. This seems difficult when random numbers are involved, because these numbers are different every time we run the algorithm, and each run hence produces a (slightly) different result. A standard way to test algorithms involving random numbers is to fix the seed of the random number generator. Then the sequence of numbers is the same every time we run the algorithm. Assuming that the MonteCarlo_double function works, we fix the seed, observe a certain result, and take this result as the correct result. Provided the test function always uses this seed, we should get exactly this result every time the MonteCarlo_double function is called. Our test function can then be written as shown below.

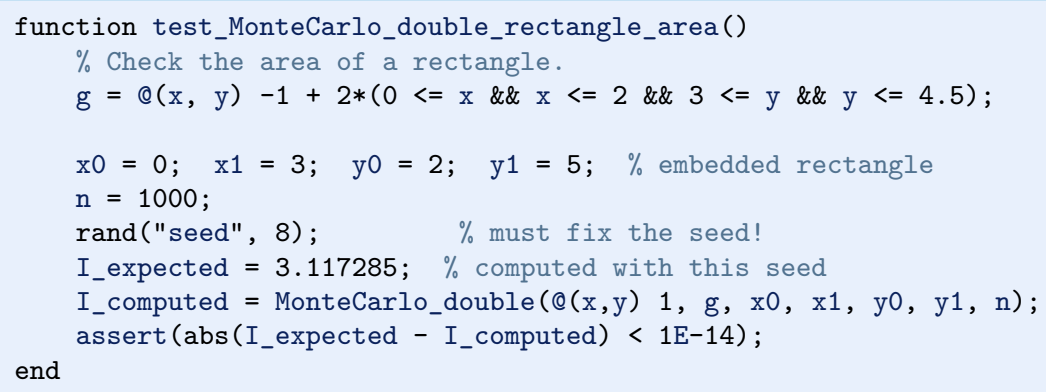

(See the file test_MonteCarlo_double_rectangle_area.m.)

Integral over a circle The test above involves a trivial function $f(x, y)=1$. We should also test a non-constant $f$ function and a more complicated domain. Let $\Omega$ be a circle at the origin with radius 2 , and let $f=\sqrt{x^{2}+y^{2}}$. This choice makes it possible to compute an exact result: in polar coordinates, $\int_{\Omega} f(x, y) d x d y$ simplifies to $2 \pi \int_{0}^{2} r^{2} d r=16 \pi / 3$. We must be prepared for quite crude approximations that fluctuate around this exact result. As in the test case above, we experience better results with larger number of points. When we have such evidence for a working implementation, we can turn the test into a proper test function. Here is an example:

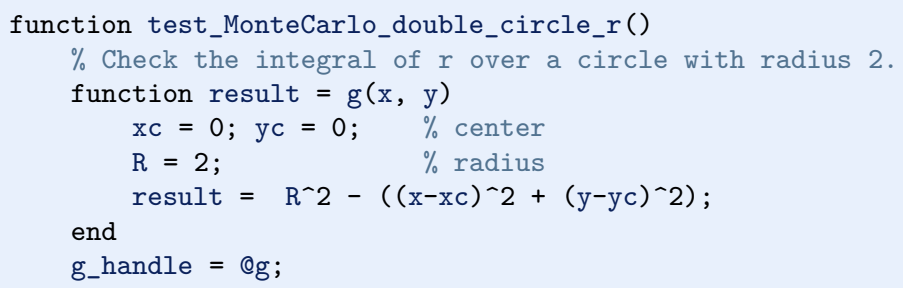




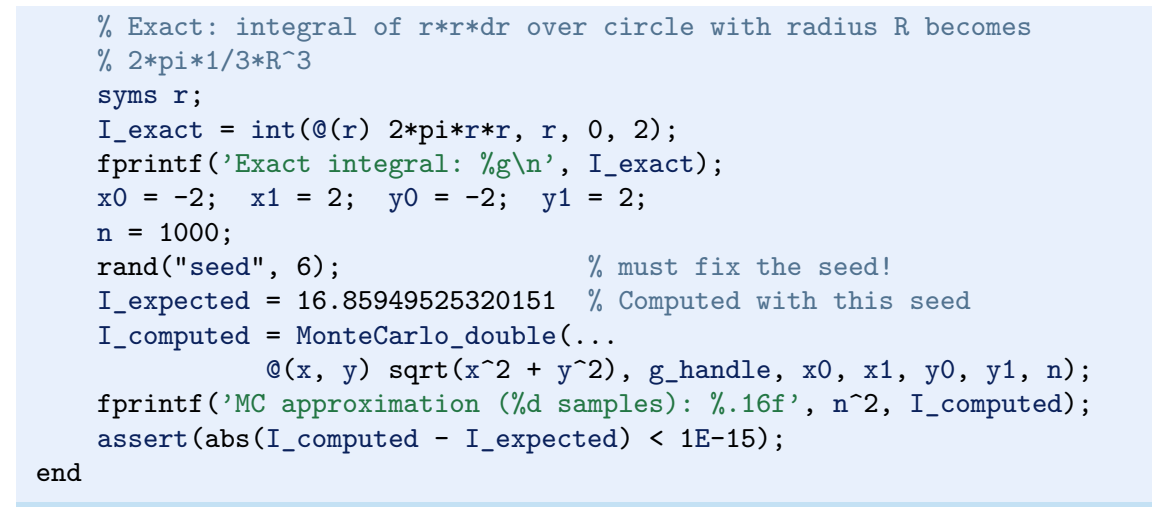

(See the file test_MonteCarlo_double_circle_r.m.)

\subsection{Exercises}

\section{Exercise 3.1: Hand calculations for the trapezoidal method}

Compute by hand the area composed of two trapezoids (of equal width) that approximates the integral $\int_{1}^{3} 2 x^{3} d x$. Make a test function that calls the trapezoidal function in trapezoidal.m and compares the return value with the hand-calculated value.

Filename: trapezoidal_test_func.m.

\section{Exercise 3.2: Hand calculations for the midpoint method}

Compute by hand the area composed of two rectangles (of equal width) that approximates the integral $\int_{1}^{3} 2 x^{3} d x$. Make a test function that calls the midpoint function in midpoint.m and compares the return value with the hand-calculated value.

Filename: midpoint_test_func.m.

\section{Exercise 3.3: Compute a simple integral}

Apply the trapezoidal and midpoint functions to compute the integral $\int_{2}^{6} x(x-$ 1) $d x$ with 2 and 100 subintervals. Compute the error too.

Filename: integrate_parabola.m.

\section{Exercise 3.4: Hand-calculations with sine integrals}

We consider integrating the sine function: $\int_{0}^{b} \sin (x) d x$.

a) Let $b=\pi$ and use two intervals in the trapezoidal and midpoint method. Compute the integral by hand and illustrate how the two numerical methods approximates the integral. Compare with the exact value.

b) Do a) when $b=2 \pi$.

Filename: integrate_sine.pdf. 


\section{Exercise 3.5: Make test functions for the midpoint method}

Modify the file test_trapezoidal.m such that the three tests are applied to the function midpoint implementing the midpoint method for integration.

Filename: test_midpoint.m.

\section{Exercise 3.6: Explore rounding errors with large numbers}

The trapezoidal method integrates linear functions exactly, and this property was used in the test function test_trapezoidal_linear in the file test_ trapezoidal.m. Change the function used in Sect. 3.4.2 to $f(x)=6 \cdot 10^{8} x-4 \cdot 10^{6}$ and rerun the test. What happens? How must you change the test to make it useful? How does the convergence rate test behave? Any need for adjustment?

Filename: test_trapezoidal2.m.

\section{Exercise 3.7: Write test functions for $\int_{0}^{4} \sqrt{x} d x$}

We want to test how the trapezoidal function works for the integral $\int_{0}^{4} \sqrt{x} d x$. Two of the tests in test_trapezoidal.m are meaningful for this integral. Compute by hand the result of using 2 or 3 trapezoids and modify the test_ trapezoidal_one_exact_result function accordingly. Then modify test_ trapezoidal_conv_rate to handle the square root integral.

Filename: test_trapezoidal3.m.

Remarks The convergence rate test fails. Printing out $r$ shows that the actual convergence rate for this integral is -1.5 and not -2 . The reason is that the error in the trapezoidal method ${ }^{6}$ is $-(b-a)^{3} n^{-2} f^{\prime \prime}(\xi)$ for some (unknown) $\xi \in[a, b]$. With $f(x)=\sqrt{x}, f^{\prime \prime}(\xi) \rightarrow-\infty$ as $\xi \rightarrow 0$, pointing to a potential problem in the size of the error. Running a test with $a>0$, say $\int_{0.1}^{4} \sqrt{x} d x$ shows that the convergence rate is indeed restored to -2 .

\section{Exercise 3.8: Rectangle methods}

The midpoint method divides the interval of integration into equal-sized subintervals and approximates the integral in each subinterval by a rectangle whose height equals the function value at the midpoint of the subinterval. Instead, one might use either the left or right end of the subinterval as illustrated in Fig. 3.4. This defines a rectangle method of integration. The height of the rectangle can be based on the left or right end or the midpoint.

a) Write a function rectangle ( $f, a, b, n$, height='left') for computing an integral $\int_{a}^{b} f(x) d x$ by the rectangle method with height computed based on the value of height, which is either left, right, or mid.

b) Write three test functions for the three unit test procedures described in Sect. 3.4.2. Make sure you test for height equal to left, right, and mid. You may call the midpoint function for checking the result when height=mid.

Hint Edit test_trapezoidal.m.

Filename: rectangle_methods.m.

${ }^{6}$ http://en.wikipedia.org/wiki/Trapezoidal_rule\#Error_analysis 

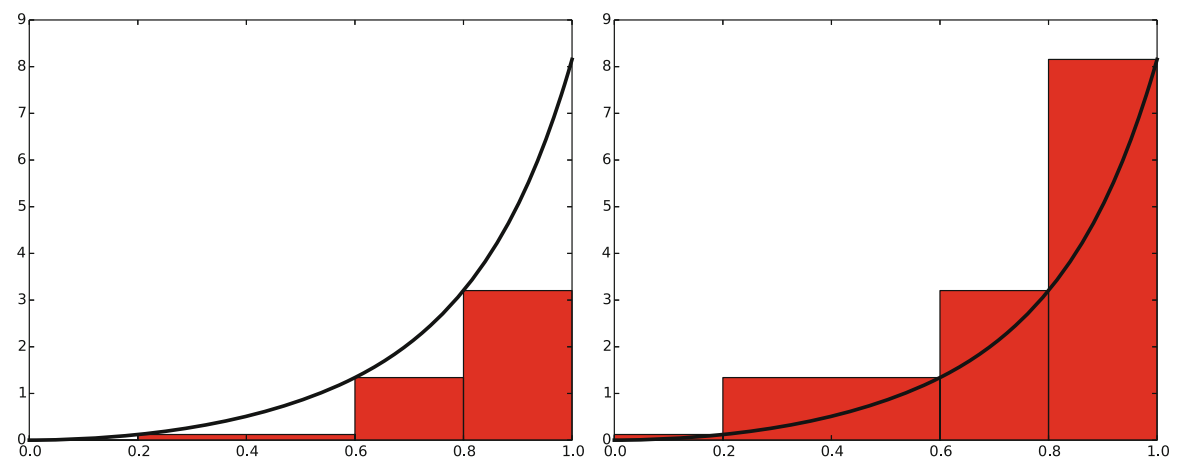

Fig. 3.4 Illustration of the rectangle method with evaluating the rectangle height by either the left or right point

\section{Exercise 3.9: Adaptive integration}

Suppose we want to use the trapezoidal or midpoint method to compute an integral $\int_{a}^{b} f(x) d x$ with an error less than a prescribed tolerance $\epsilon$. What is the appropriate size of $n$ ?

To answer this question, we may enter an iterative procedure where we compare the results produced by $n$ and $2 n$ intervals, and if the difference is smaller than $\epsilon$, the value corresponding to $2 n$ is returned. Otherwise, we halve $n$ and repeat the procedure.

Hint It may be a good idea to organize your code so that the function adaptive integration can be used easily in future programs you write.

a) Write a function

adaptive_integration( $f, a, b$, eps, method=midpoint)

that implements the idea above (eps corresponds to the tolerance $\epsilon$, and method can be midpoint or trapezoidal).

b) Test the method on $\int_{0}^{2} x^{2} d x$ and $\int_{0}^{2} \sqrt{x} d x$ for $\epsilon=10^{-1}, 10^{-10}$ and write out the exact error.

c) Make a plot of $n$ versus $\epsilon \in\left[10^{-1}, 10^{-10}\right]$ for $\int_{0}^{2} \sqrt{x} d x$. Use logarithmic scale for $\epsilon$.

Filename: adaptive_integration.m.

Remarks The type of method explored in this exercise is called adaptive, because it tries to adapt the value of $n$ to meet a given error criterion. The true error can very seldom be computed (since we do not know the exact answer to the computational problem), so one has to find other indicators of the error, such as the one here where the changes in the integral value, as the number of intervals is doubled, is taken to reflect the error. 


\section{Exercise 3.10: Integrating $x$ raised to $x$}

Consider the integral

$$
I=\int_{0}^{4} x^{x} d x .
$$

The integrand $x^{x}$ does not have an anti-derivative that can be expressed in terms of standard functions (visit http: //wolframalpha.comand type integral ( $\mathrm{x}^{\wedge} \mathrm{x}, \mathrm{x}$ ) to convince yourself that our claim is right. Note that Wolfram alpha does give you an answer, but that answer is an approximation, it is not exact. This is because Wolfram alpha too uses numerical methods to arrive at the answer, just as you will in this exercise). Therefore, we are forced to compute the integral by numerical methods. Compute a result that is right to four digits.

Hint Use ideas from Exercise 3.9.

Filename: integrate_x $2 x \cdot m$.

Exercise 3.11: Integrate products of sine functions

In this exercise we shall integrate

$$
I_{j, k}=\int_{-\pi}^{\pi} \sin (j x) \sin (k x) d x,
$$

where $j$ and $k$ are integers.

a) Plot $\sin (x) \sin (2 x)$ and $\sin (2 x) \sin (3 x)$ for $x \in]-\pi, \pi]$ in separate plots. Explain why you expect $\int_{-\pi}^{\pi} \sin x \sin 2 x d x=0$ and $\int_{-\pi}^{\pi} \sin 2 x \sin 3 x d x=0$.

b) Use the trapezoidal rule to compute $I_{j, k}$ for $j=1, \ldots, 10$ and $k=1, \ldots, 10$.

Filename: products_sines.m.

\section{Exercise 3.12: Revisit fit of sines to a function}

This is a continuation of Exercise 2.18. The task is to approximate a given function $f(t)$ on $[-\pi, \pi]$ by a sum of sines,

$$
S_{N}(t)=\sum_{n=1}^{N} b_{n} \sin (n t) .
$$

We are now interested in computing the unknown coefficients $b_{n}$ such that $S_{N}(t)$ is in some sense the best approximation to $f(t)$. One common way of doing this is to first set up a general expression for the approximation error, measured by "summing up" the squared deviation of $S_{N}$ from $f$ :

$$
E=\int_{-\pi}^{\pi}\left(S_{N}(t)-f(t)\right)^{2} d t .
$$


We may view $E$ as a function of $b_{1}, \ldots, b_{N}$. Minimizing $E$ with respect to $b_{1}, \ldots, b_{N}$ will give us a best approximation, in the sense that we adjust $b_{1}, \ldots, b_{N}$ such that $S_{N}$ deviates as little as possible from $f$.

Minimization of a function of $N$ variables, $E\left(b_{1}, \ldots, b_{N}\right)$ is mathematically performed by requiring all the partial derivatives to be zero:

$$
\begin{gathered}
\frac{\partial E}{\partial b_{1}}=0, \\
\frac{\partial E}{\partial b_{2}}=0, \\
\vdots \\
\frac{\partial E}{\partial b_{N}}=0 .
\end{gathered}
$$

a) Compute the partial derivative $\partial E / \partial b_{1}$ and generalize to the arbitrary case $\partial E / \partial b_{n}, 1 \leq n \leq N$

b) Show that

$$
b_{n}=\frac{1}{\pi} \int_{-\pi}^{\pi} f(t) \sin (n t) d t .
$$

c) Write a function integrate_coeffs ( $f, N, M)$ that computes $b_{1}, \ldots, b_{N}$ by numerical integration, using $M$ intervals in the trapezoidal rule.

d) A remarkable property of the trapezoidal rule is that it is exact for integrals $\int_{-\pi}^{\pi} \sin n t d t$ (when subintervals are of equal size). Use this property to create a function test_integrate_coeff to verify the implementation of integrate_coeffs.

e) Implement the choice $f(t)=\frac{1}{\pi} t$ as a Matlab function $\mathrm{f}(\mathrm{t})$ and call integrate_coeffs $(f, 3,100)$ to see what the optimal choice of $b_{1}, b_{2}, b_{3}$ is.

f) Make a function plot_approx $(f, N, M$, filename) where you plot $f(t)$ together with the best approximation $S_{N}$ as computed above, using $M$ intervals for numerical integration. Save the plot to a file with name $f$ ilename.

g) Runplot_approx(f, N, M, filename) for $f(t)=\frac{1}{\pi} t$ for $N=3,6,12,24$. Observe how the approximation improves.

h) Run plot_approx for $f(t)=e^{-(t-\pi)}$ and $N=100$. Observe a fundamental problem: regardless of $N, S_{N}(-\pi)=0$, not $e^{2 \pi} \approx 535$. (There are ways to fix this issue.)

Filename: autofit_sines.m.

\section{Exercise 3.13: Derive the trapezoidal rule for a double integral}

Use ideas in Sect. 3.7.1 to derive a formula for computing a double integral $\int_{a}^{b} \int_{c}^{d} f(x, y) d y d x$ by the trapezoidal rule. Implement and test this rule.

Filename: trapezoidal_double.m.

\section{Exercise 3.14: Compute the area of a triangle by Monte Carlo integration}

Use the Monte Carlo method from Sect. 3.7.3 to compute the area of a triangle with vertices at $(-1,0),(1,0)$, and $(3,0)$.

Filename: MC_triangle.m. 
Open Access This chapter is distributed under the terms of the Creative Commons AttributionNonCommercial 4.0 International License (http://creativecommons.org/licenses/by-nc/4.0/), which permits any noncommercial use, duplication, adaptation, distribution and reproduction in any medium or format, as long as you give appropriate credit to the original author(s) and the source, a link is provided to the Creative Commons license and any changes made are indicated.

The images or other third party material in this chapter are included in the work's Creative Commons license, unless indicated otherwise in the credit line; if such material is not included in the work's Creative Commons license and the respective action is not permitted by statutory regulation, users will need to obtain permission from the license holder to duplicate, adapt or reproduce the material. 


\section{Solving Ordinary Differential Equations}

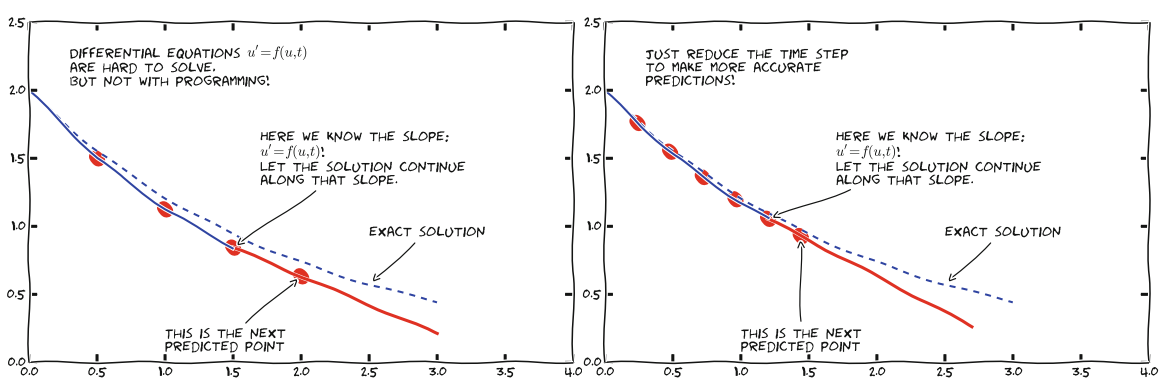

Differential equations constitute one of the most powerful mathematical tools to understand and predict the behavior of dynamical systems in nature, engineering, and society. A dynamical system is some system with some state, usually expressed by a set of variables, that evolves in time. For example, an oscillating pendulum, the spreading of a disease, and the weather are examples of dynamical systems. We can use basic laws of physics, or plain intuition, to express mathematical rules that govern the evolution of the system in time. These rules take the form of differential equations. You are probably well experienced with equations, at least equations like $a x+b=0$ or $a x^{2}+b x+c=0$. Such equations are known as algebraic equations, and the unknown is a number. The unknown in a differential equation is a function, and a differential equation will almost always involve this function and one or more derivatives of the function. For example, $f^{\prime}(x)=f(x)$ is a simple differential equation (asking if there is any function $f$ such that it equals its derivative - you might remember that $e^{x}$ is a candidate).

The present chapter starts with explaining how easy it is to solve both single (scalar) first-order ordinary differential equations and systems of first-order differential equations by the Forward Euler method. We demonstrate all the mathematical and programming details through two specific applications: population growth and spreading of diseases.

Then we turn to a physical application: oscillating mechanical systems, which arise in a wide range of engineering situations. The differential equation is now of second order, and the Forward Euler method does not perform well. This observation motivates the need for other solution methods, and we derive the Euler-Cromer 
scheme $^{1}$, the 2 nd- and 4th-order Runge-Kutta schemes, as well as a finite difference scheme (the latter to handle the second-order differential equation directly without reformulating it as a first-order system). The presentation starts with undamped free oscillations and then treats general oscillatory systems with possibly nonlinear damping, nonlinear spring forces, and arbitrary external excitation. Besides developing programs from scratch, we also demonstrate how to access ready-made implementations of more advanced differential equation solvers in Matlab.

As we progress with more advanced methods, we develop more sophisticated and reusable programs, and in particular, we incorporate good testing strategies so that we bring solid evidence to correct computations. Consequently, the beginning with population growth and disease modeling examples has a very gentle learning curve, while that curve gets significantly steeper towards the end of the treatment of differential equations for oscillatory systems.

\subsection{Population Growth}

Our first taste of differential equations regards modeling the growth of some population, such as a cell culture, an animal population, or a human population. The ideas even extend trivially to growth of money in a bank. Let $N(t)$ be the number of individuals in the population at time $t$. How can we predict the evolution of $N(t)$ in time? Below we shall derive a differential equation whose solution is $N(t)$. The equation reads

$$
N^{\prime}(t)=r N(t),
$$

where $r$ is a number. Note that although $N$ is an integer in real life, we model $N$ as a real-valued function. We are forced to do this because the solution of differential equations are (normally continuous) real-valued functions. An integer-valued $N(t)$ in the model would lead to a lot of mathematical difficulties.

With a bit of guessing, you may realize that $N(t)=C e^{r t}$, where $C$ is any number. To make this solution unique, we need to fix $C$, done by prescribing the value of $N$ at some time, usually $t=0$. Say $N(0)$ is given as $N_{0}$. Then $N(t)=$ $N_{0} e^{r t}$.

In general, a differential equation model consists of a differential equation, such as (4.1) and an initial condition, such as $N(0)=N_{0}$. With a known initial condition, the differential equation can be solved for the unknown function and the solution is unique.

It is, of course, very seldom that we can find the solution of a differential equation as easy as in this example. Normally, one has to apply certain mathematical methods, but these can only handle some of the simplest differential equations. However, we can easily deal with almost any differential equation by applying numerical methods and a bit of programming. This is exactly the topic of the present chapter.

\footnotetext{
${ }^{1}$ The term scheme is used as synonym for method or computational recipe, especially in the context of numerical methods for differential equations.
} 


\subsubsection{Derivation of the Model}

It can be instructive to show how an equation like (4.1) arises. Consider some population of (say) an animal species and let $N(t)$ be the number of individuals in a certain spatial region, e.g. an island. We are not concerned with the spatial distribution of the animals, just the number of them in some spatial area where there is no exchange of individuals with other spatial areas. During a time interval $\Delta t$, some animals will die and some new will be born. The number of deaths and births are expected to be proportional to $N$. For example, if there are twice as many individuals, we expect them to get twice as many newborns. In a time interval $\Delta t$, the net growth of the population will be

$$
N(t+\Delta t)-N(t)=\bar{b} N(t)-\bar{d} N(t)
$$

where $\bar{b} N(t)$ is the number of newborns and $\bar{d} N(t)$ is the number of deaths. If we double $\Delta t$, we expect the proportionality constants $\bar{b}$ and $\bar{d}$ to double too, so it makes sense to think of $\bar{b}$ and $\bar{d}$ as proportional to $\Delta t$ and "factor out" $\Delta t$. That is, we introduce $b=\bar{b} / \Delta t$ and $d=\bar{d} / \Delta t$ to be proportionality constants for newborns and deaths independent of $\Delta t$. Also, we introduce $r=b-d$, which is the net rate of growth of the population per time unit. Our model then becomes

$$
N(t+\Delta t)-N(t)=\Delta t r N(t) .
$$

Equation (4.2) is actually a computational model. Given $N(t)$, we can advance the population size by

$$
N(t+\Delta t)=N(t)+\Delta t r N(t) .
$$

This is called a difference equation. If we know $N(t)$ for some $t$, e.g., $N(0)=N_{0}$, we can compute

$$
\begin{aligned}
N(\Delta t) & =N_{0}+\Delta t r N_{0}, \\
N(2 \Delta t) & =N(\Delta t)+\Delta t r N(\Delta t), \\
N(3 \Delta t) & =N(2 \Delta t)+\Delta t r N(2 \Delta t), \\
& \vdots \\
N((k+1) \Delta t) & =N(k \Delta t)+\Delta t r N(k \Delta t),
\end{aligned}
$$

where $k$ is some arbitrary integer. A computer program can easily compute $N((k+$ 1) $\Delta t$ ) for us with the aid of a little loop.

\section{Warning}

Observe that the computational formula cannot be started unless we have an initial condition!

The solution of $N^{\prime}=r N$ is $N=C e^{r t}$ for any constant $C$, and the initial condition is needed to fix $C$ so the solution becomes unique. However, from a mathematical point of view, knowing $N(t)$ at any point $t$ is sufficient as initial 
condition. Numerically, we more literally need an initial condition: we need to know a starting value at the left end of the interval in order to get the computational formula going.

In fact, we do not need a computer since we see a repetitive pattern when doing hand calculations, which leads us to a mathematical formula for $N((k+1) \Delta t)$ :

$$
\begin{aligned}
N((k+1) \Delta t) & =N(k \Delta t)+\Delta t r N(k \Delta t)=N(k \Delta t)(1+\Delta t r) \\
& =N((k-1) \Delta t)(1+\Delta t r)^{2} \\
& \vdots \\
& =N_{0}(1+\Delta t r)^{k+1} .
\end{aligned}
$$

Rather than using (4.2) as a computational model directly, there is a strong tradition for deriving a differential equation from this difference equation. The idea is to consider a very small time interval $\Delta t$ and look at the instantaneous growth as this time interval is shrunk to an infinitesimally small size. In mathematical terms, it means that we let $\Delta t \rightarrow 0$. As (4.2) stands, letting $\Delta t \rightarrow 0$ will just produce an equation $0=0$, so we have to divide by $\Delta t$ and then take the limit:

$$
\lim _{\Delta t \rightarrow 0} \frac{N(t+\Delta t)-N(t)}{\Delta t}=r N(t)
$$

The term on the left-hand side is actually the definition of the derivative $N^{\prime}(t)$, so we have

$$
N^{\prime}(t)=r N(t)
$$

which is the corresponding differential equation.

There is nothing in our derivation that forces the parameter $r$ to be constant it can change with time due to, e.g., seasonal changes or more permanent environmental changes.

\section{Detour: Exact mathematical solution}

If you have taken a course on mathematical solution methods for differential equations, you may want to recap how an equation like $N^{\prime}=r N$ or $N^{\prime}=r(t) N$ is solved. The method of separation of variables is the most convenient solution strategy in this case:

$$
\begin{aligned}
N^{\prime} & =r N \\
\frac{d N}{d t} & =r N \\
\frac{d N}{N} & =r d t \\
\int_{N_{0}}^{N} \frac{d N}{N} & =\int_{0}^{t} r d t
\end{aligned}
$$




$$
\begin{aligned}
\ln N-\ln N_{0} & =\int_{0}^{t} r(t) d t \\
N & =N_{0} \exp \left(\int_{0}^{t} r(t) d t\right),
\end{aligned}
$$

which for constant $r$ results in $N=N_{0} e^{r t}$. Note that $\exp (t)$ is the same as $e^{t}$.

As will be described later, $r$ must in more realistic models depend on $N$. The method of separation of variables then requires to integrate $\int_{N_{0}}^{N} N / r(N) d N$, which quickly becomes non-trivial for many choices of $r(N)$. The only generally applicable solution approach is therefore a numerical method.

\subsubsection{Numerical Solution}

There is a huge collection of numerical methods for problems like (4.2), and in general any equation of the form $u^{\prime}=f(u, t)$, where $u(t)$ is the unknown function in the problem, and $f$ is some known formula of $u$ and optionally $t$. For example, $f(u, t)=r u$ in (4.2). We will first present a simple finite difference method solving $u^{\prime}=f(u, t)$. The idea is four-fold:

1. Introduce a mesh in time with $N_{t}+1$ points $t_{0}, t_{1}, \ldots, t_{N_{t}}$. We seek the unknown $u$ at the mesh points $t_{n}$, and introduce $u^{n}$ as the numerical approximation to $u\left(t_{n}\right)$, see Fig. 4.1 .

2. Assume that the differential equation is valid at the mesh points.

3. Approximate derivatives by finite differences, see Fig. 4.2.

4. Formulate a computational algorithm that can compute a new value $u^{n}$ based on previously computed values $u^{i}, i<n$.

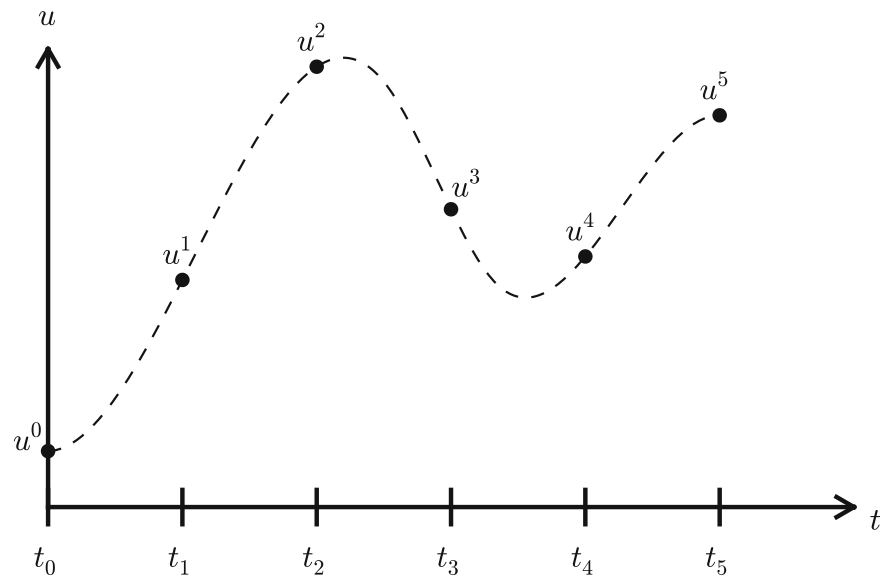

Fig. 4.1 Mesh in time with corresponding discrete values (unknowns) 


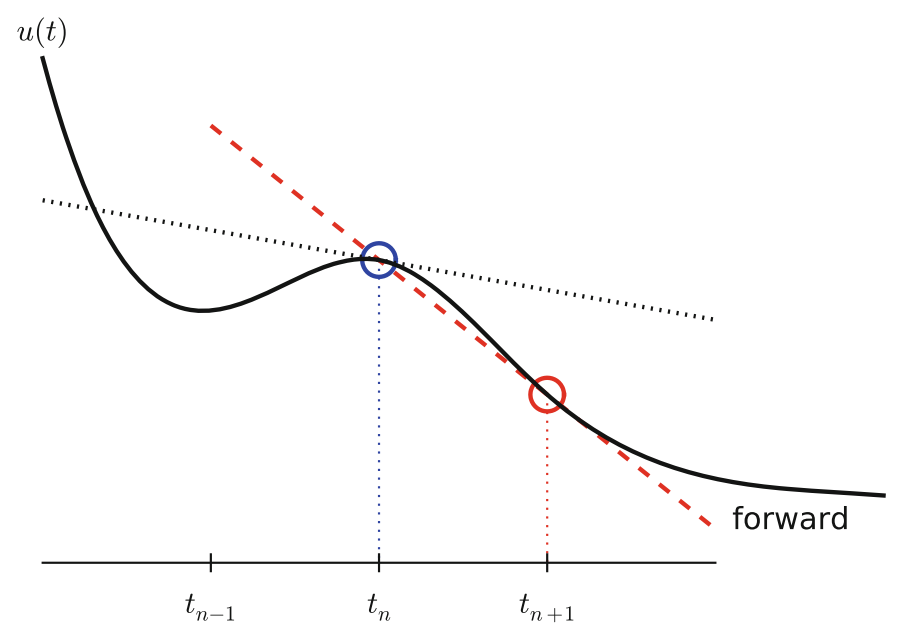

Fig. 4.2 Illustration of a forward difference approximation to the derivative

An example will illustrate the steps. First, we introduce the mesh, and very often the mesh is uniform, meaning that the spacing between points $t_{n}$ and $t_{n+1}$ is constant. This property implies that

$$
t_{n}=n \Delta t, \quad n=0,1, \ldots, N_{t} .
$$

Second, the differential equation is supposed to hold at the mesh points. Note that this is an approximation, because the differential equation is originally valid at all real values of $t$. We can express this property mathematically as

$$
u^{\prime}\left(t_{n}\right)=f\left(u^{n}, t_{n}\right), \quad n=0,1, \ldots, N_{t} .
$$

For example, with our model equation $u^{\prime}=r u$, we have the special case

$$
u^{\prime}\left(t_{n}\right)=r u^{n}, \quad n=0,1, \ldots, N_{t},
$$

or

$$
u^{\prime}\left(t_{n}\right)=r\left(t_{n}\right) u^{n}, \quad n=0,1, \ldots, N_{t},
$$

if $r$ depends explicitly on $t$.

Third, derivatives are to be replaced by finite differences. To this end, we need to know specific formulas for how derivatives can be approximated by finite differences. One simple possibility is to use the definition of the derivative from any calculus book,

$$
u^{\prime}(t)=\lim _{\Delta t \rightarrow 0} \frac{u(t+\Delta t)-u(t)}{\Delta t} .
$$

At an arbitrary mesh point $t_{n}$ this definition can be written as

$$
u^{\prime}\left(t_{n}\right)=\lim _{\Delta t \rightarrow 0} \frac{u^{n+1}-u^{n}}{\Delta t} .
$$


Instead of going to the limit $\Delta t \rightarrow 0$ we can use a small $\Delta t$, which yields a computable approximation to $u^{\prime}\left(t_{n}\right)$ :

$$
u^{\prime}\left(t_{n}\right) \approx \frac{u^{n+1}-u^{n}}{\Delta t} .
$$

This is known as a forward difference since we go forward in time $\left(u^{n+1}\right)$ to collect information in $u$ to estimate the derivative. Figure 4.2 illustrates the idea. The error of the forward difference is proportional to $\Delta t$ (often written as $\mathcal{O}(\Delta t)$, but we will not use this notation in the present book).

We can now plug in the forward difference in our differential equation sampled at the arbitrary mesh point $t_{n}$ :

$$
\frac{u^{n+1}-u^{n}}{\Delta t}=f\left(u^{n}, t_{n}\right)
$$

or with $f(u, t)=r u$ in our special model problem for population growth,

$$
\frac{u^{n+1}-u^{n}}{\Delta t}=r u^{n} .
$$

If $r$ depends on time, we insert $r\left(t_{n}\right)=r^{n}$ for $r$ in this latter equation.

The fourth step is to derive a computational algorithm. Looking at (4.3), we realize that if $u^{n}$ should be known, we can easily solve with respect to $u^{n+1}$ to get a formula for $u$ at the next time level $t_{n+1}$ :

$$
u^{n+1}=u^{n}+\Delta t f\left(u^{n}, t_{n}\right)
$$

Provided we have a known starting value, $u^{0}=U_{0}$, we can use (4.5) to advance the solution by first computing $u^{1}$ from $u^{0}$, then $u^{2}$ from $u^{1}, u^{3}$ from $u^{2}$, and so forth.

Such an algorithm is called a numerical scheme for the differential equation and often written compactly as

$$
u^{n+1}=u^{n}+\Delta t f\left(u^{n}, t_{n}\right), \quad u^{0}=U_{0}, \quad n=0,1, \ldots, N_{t}-1 .
$$

This scheme is known as the Forward Euler scheme, also called Euler's method.

In our special population growth model, we have

$$
u^{n+1}=u^{n}+\Delta t r u^{n}, \quad u^{0}=U_{0}, \quad n=0,1, \ldots, N_{t}-1 .
$$

We may also write this model using the problem-specific symbol $N$ instead of the generic $u$ function:

$$
N^{n+1}=N^{n}+\Delta t r N^{n}, \quad N^{0}=N_{0}, \quad n=0,1, \ldots, N_{t}-1 .
$$

The observant reader will realize that (4.8) is nothing but the computational model (4.2) arising directly in the model derivation. The formula (4.8) arises, however, from a detour via a differential equation and a numerical method for the differential equation. This looks rather unnecessary! The reason why we bother to 


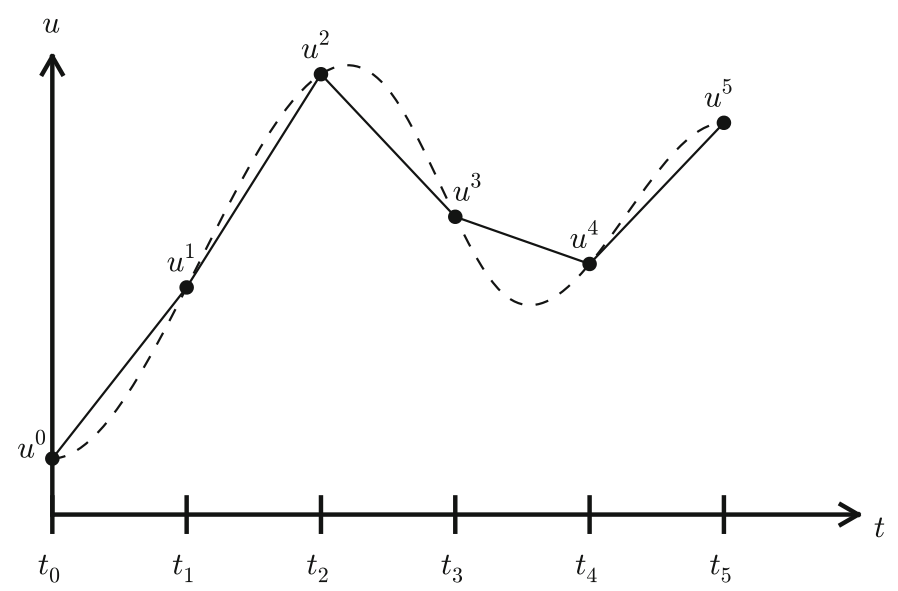

Fig. 4.3 The numerical solution at points can be extended by linear segments between the mesh points

derive the differential equation model and then discretize it by a numerical method is simply that the discretization can be done in many ways, and we can create (much) more accurate and more computationally efficient methods than (4.8) or (4.6). This can be useful in many problems! Nevertheless, the Forward Euler scheme is intuitive and widely applicable, at least when $\Delta t$ is chosen to be small.

\section{The numerical solution between the mesh points}

Our numerical method computes the unknown function $u$ at discrete mesh points $t_{1}, t_{2}, \ldots, t_{N_{t}}$. What if we want to evaluate the numerical solution between the mesh points? The most natural choice is to assume a linear variation between the mesh points, see Fig. 4.3. This is compatible with the fact that when we plot the array $u^{0}, u^{1}, \ldots$ versus $t_{0}, t_{1}, \ldots$, a straight line is drawn between the discrete points.

\subsubsection{Programming the Forward Euler Scheme; the Special Case}

Let us compute (4.8) in a program. The input variables are $N_{0}, \Delta t, r$, and $N_{t}$. Note that we need to compute $N_{t}+1$ new values $N^{1}, \ldots, N^{N_{t}+1}$. A total of $N_{t}+2$ values are needed in an array representation of $N^{n}, n=0, \ldots, N_{t}+1$.

Our first version of this program is as simple as possible:

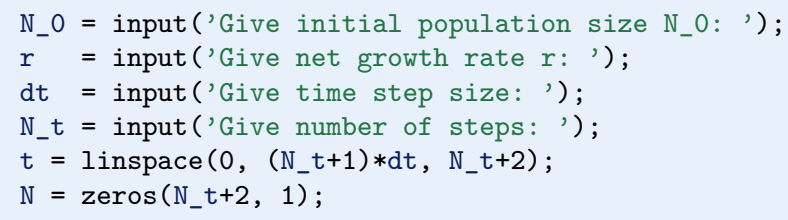




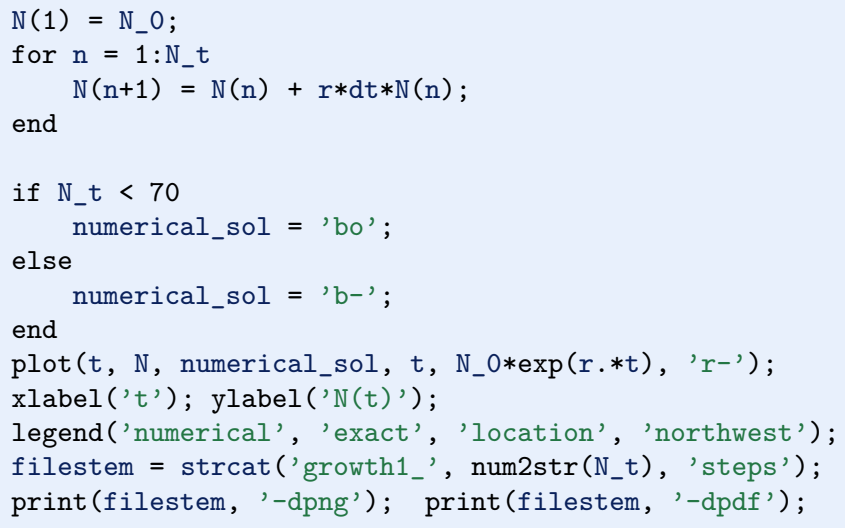

The complete code above resides in the file growth $1 . \mathrm{m}$.

Let us demonstrate a simulation where we start with 100 animals, a net growth rate of 10 percent $(0.1)$ per time unit, which can be one month, and $t \in[0,20]$ months. We may first try $\Delta t$ of half a month (0.5), which implies $N_{t}=40$ (or to be absolutely precise, the last time point to be computed according to our set-up above is $t_{N_{t}+1}=20.5$ ). Figure 4.4 shows the results. The solid line is the exact solution, while the circles are the computed numerical solution. The discrepancy is clearly visible. What if we make $\Delta t 10$ times smaller? The result is displayed in Fig. 4.5, where we now use a solid line also for the numerical solution (otherwise, 400 circles would look very cluttered, so the program has a test on how to display the numerical solution, either as circles or a solid line). We can hardly distinguish the exact and the numerical solution. The computing time is also a fraction of

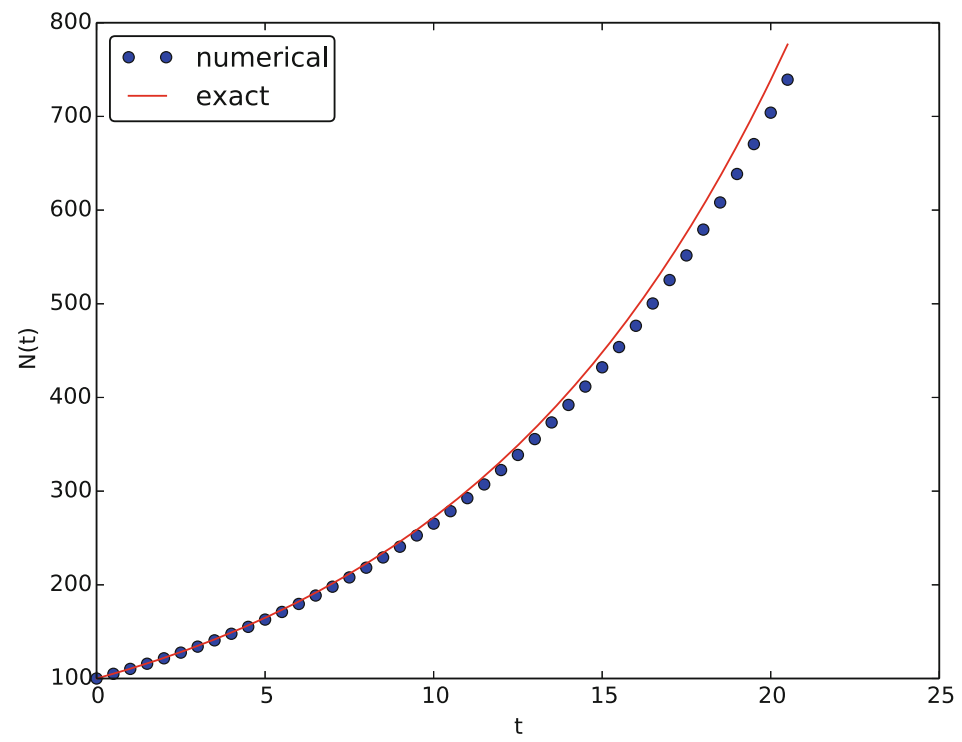

Fig.4.4 Evolution of a population computed with time step 0.5 month 


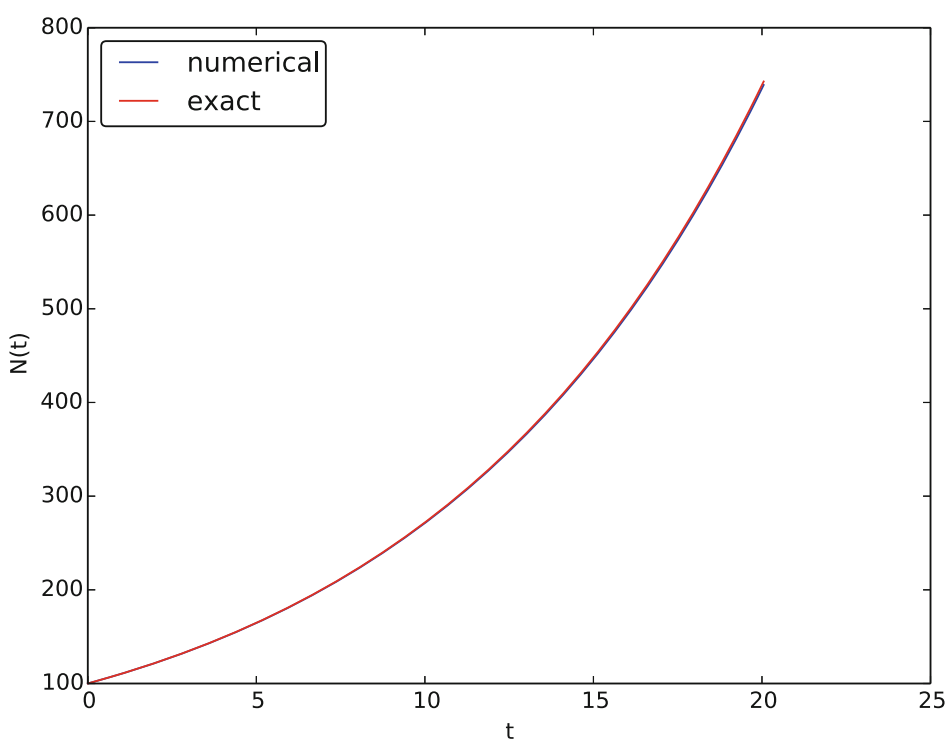

Fig. 4.5 Evolution of a population computed with time step 0.05 month

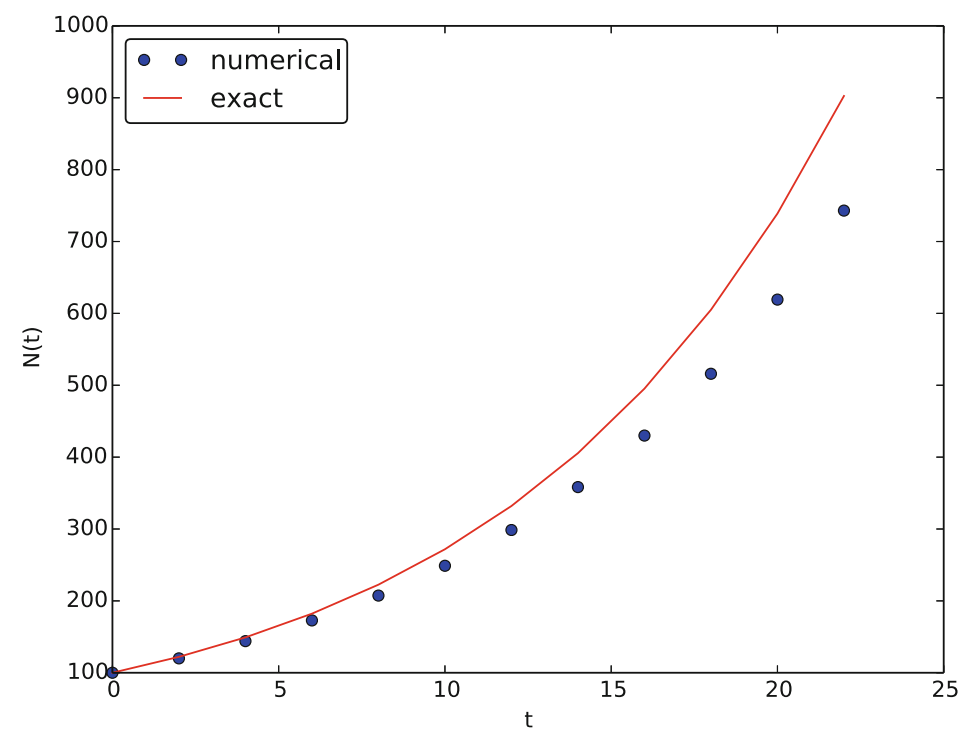

Fig. 4.6 Evolution of a population computed with time step 2 months

a second on a laptop, so it appears that the Forward Euler method is sufficiently accurate for practical purposes. (This is not always true for large, complicated simulation models in engineering, so more sophisticated methods may be needed.)

It is also of interest to see what happens if we increase $\Delta t$ to 2 months. The results in Fig. 4.6 indicate that this is an inaccurate computation. 


\subsubsection{Understanding the Forward Euler Method}

The good thing about the Forward Euler method is that it gives an understanding of what a differential equation is and a geometrical picture of how to construct the solution. The first idea is that we have already computed the solution up to some time point $t_{n}$. The second idea is that we want to progress the solution from $t_{n}$ to $t_{n+1}$ as a straight line.

We know that the line must go through the solution at $t_{n}$, i.e., the point $\left(t_{n}, u^{n}\right)$. The differential equation tells us the slope of the line: $u^{\prime}\left(t_{n}\right)=f\left(u^{n}, t_{n}\right)=r u^{n}$. That is, the differential equation gives a direct formula for the further direction of the solution curve. We can say that the differential equation expresses how the system $(u)$ undergoes changes at a point.

There is a general formula for a straight line $y=a x+b$ with slope $a$ that goes through the point $\left(x_{0}, y_{0}\right): y=a\left(x-x_{0}\right)+y_{0}$. Using this formula adapted to the present case, and evaluating the formula for $t_{n+1}$, results in

$$
u^{n+1}=r u^{n}\left(t_{n+1}-t_{n}\right)+u^{n}=u^{n}+\Delta t r u^{n},
$$

which is nothing but the Forward Euler formula. You are now encouraged to do Exercise 4.1 to become more familiar with the geometric interpretation of the Forward Euler method.

\subsubsection{Programming the Forward Euler Scheme; the General Case}

Our previous program was just a flat main program tailored to a special differential equation. When programming mathematics, it is always good to consider a (large) class of problems and making a Matlab function to solve any problem that fits into the class. More specifically, we will make software for the class of differential equation problems of the form

$$
u^{\prime}(t)=f(u, t), \quad u=U_{0}, t \in[0, T]
$$

for some given function $f$, and numbers $U_{0}$ and $T$. We also take the opportunity to illustrate what is commonly called a demo function. As the name implies, the purpose of such a function is solely to demonstrate how the function works (not to be confused with a test function, which does verification by use of assert). The Matlab function calculating the solution must take $f, U_{0}, \Delta t$, and $T$ as input, find the corresponding $N_{t}$, compute the solution, and return an array with $u^{0}, u^{1}, \ldots, u^{N_{t}}$ and an array with $t_{0}, t_{1}, \ldots, t_{N_{t}}$. The Forward Euler scheme reads

$$
u^{n+1}=u^{n}+\Delta t f\left(u^{n}, t_{n}\right), \quad n=0, \ldots, N_{t}-1 .
$$

The corresponding program ode_FE.m may now take the form

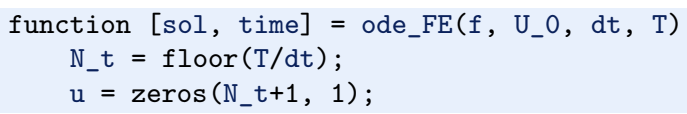




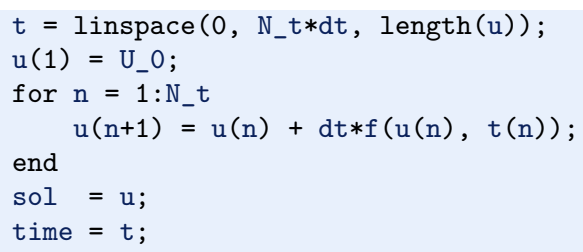

Note that the function ode_FE is general, i.e. it can solve any single differential equation $u^{\prime}=f(u, t)$. However, we will soon (in Sect. 4.2.6) generalize this ode_FE function to handle a system of multiple odes, where a single ode is just a special case (this most general version of ode_FE is what we actually store in the file ode_FE.m).

A proper demo function for this solver might be written as (file demo_ population_growth.m):

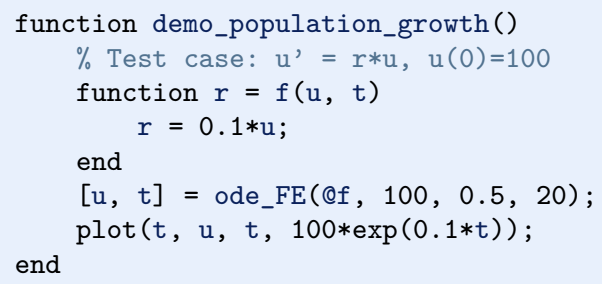

The solution should be identical to what the growth1.m program produces with the same parameter settings $\left(r=0.1, N_{0}=100\right)$. This feature can easily be tested by inserting a print statement, but a much better, automated verification is suggested in Exercise 4.1. You are strongly encouraged to take a "break" and do that exercise now.

\section{Remark on the use of $u$ as variable}

In the ode_FE program, the variable $u$ is used in different contexts. Inside the ode_FE function, $u$ is an array, but in the $f(u, t)$ function, as exemplified in the demo_population_growth function, the argument $u$ is a number. Typically, we call $f$ (in ode_FE) with the $u$ argument as one element of the array $u$ in the ode_FE function: $u(n)$.

\subsubsection{Making the Population Growth Model More Realistic}

Exponential growth of a population according the model $N^{\prime}=r N$, with exponential solution $N=N_{0} e^{r t}$, is unrealistic in the long run because the resources needed to feed the population are finite. At some point there will not be enough resources and the growth will decline. A common model taking this effect into account assumes that $r$ depends on the size of the population, $N$ :

$$
N(t+\Delta t)-N(t)=r(N(t)) N(t) .
$$


The corresponding differential equation becomes

$$
N^{\prime}=r(N) N \text {. }
$$

The reader is strongly encouraged to repeat the steps in the derivation of the Forward Euler scheme and establish that we get

$$
N^{n+1}=N^{n}+\Delta \operatorname{tr}\left(N^{n}\right) N^{n},
$$

which computes as easy as for a constant $r$, since $r\left(N^{n}\right)$ is known when computing $N^{n+1}$. Alternatively, one can use the Forward Euler formula for the general problem $u^{\prime}=f(u, t)$ and use $f(u, t)=r(u) u$ and replace $u$ by $N$.

The simplest choice of $r(N)$ is a linear function, starting with some growth value $\bar{r}$ and declining until the population has reached its maximum, $M$, according to the available resources:

$$
r(N)=\bar{r}(1-N / M) .
$$

In the beginning, $N \ll M$ and we will have exponential growth $e^{\bar{r} t}$, but as $N$ increases, $r(N)$ decreases, and when $N$ reaches $M, r(N)=0$ so there is now more growth and the population remains at $N(t)=M$. This linear choice of $r(N)$ gives rise to a model that is called the logistic model. The parameter $M$ is known as the carrying capacity of the population.

Let us run the logistic model with aid of the ode_FE function. We choose $N(0)=100, \Delta t=0.5$ month, $T=60$ months, $r=0.1$, and $M=500$. The complete program, called logistic.m, is basically a call to ode_FE:

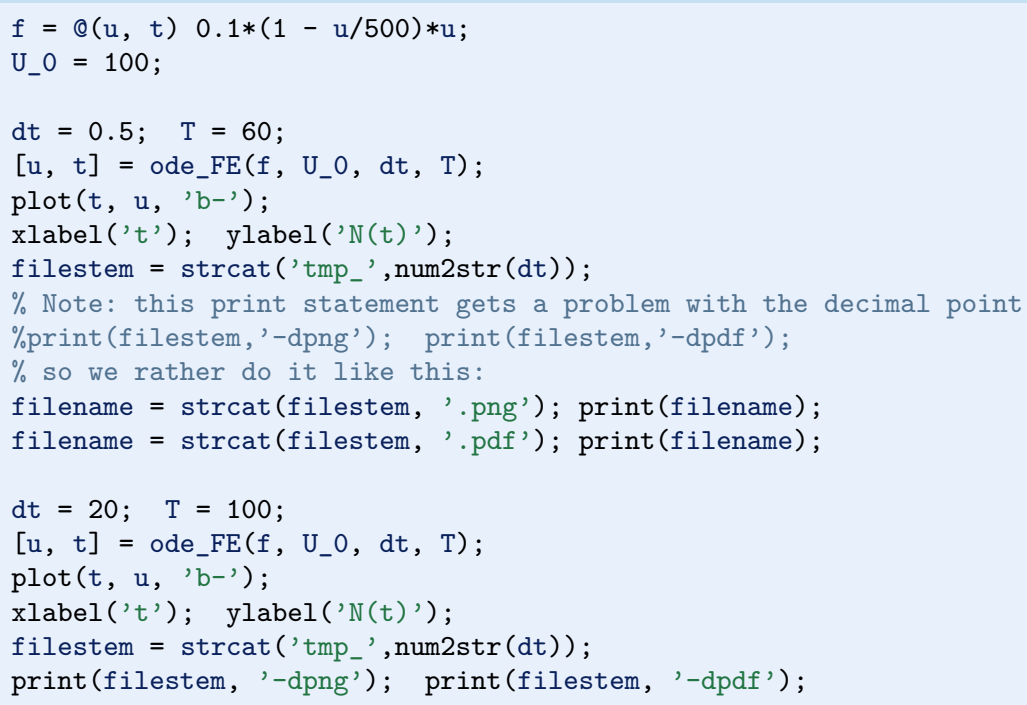

Figure 4.7 shows the resulting curve. We see that the population stabilizes around $M=500$ individuals. A corresponding exponential growth would reach $N_{0} e^{r t}=100 e^{0.1 \cdot 60} \approx 40,300$ individuals!

It is always interesting to see what happens with large $\Delta t$ values. We may set $\Delta t=20$ and $T=100$. Now the solution, seen in Fig. 4.8, oscillates and is 


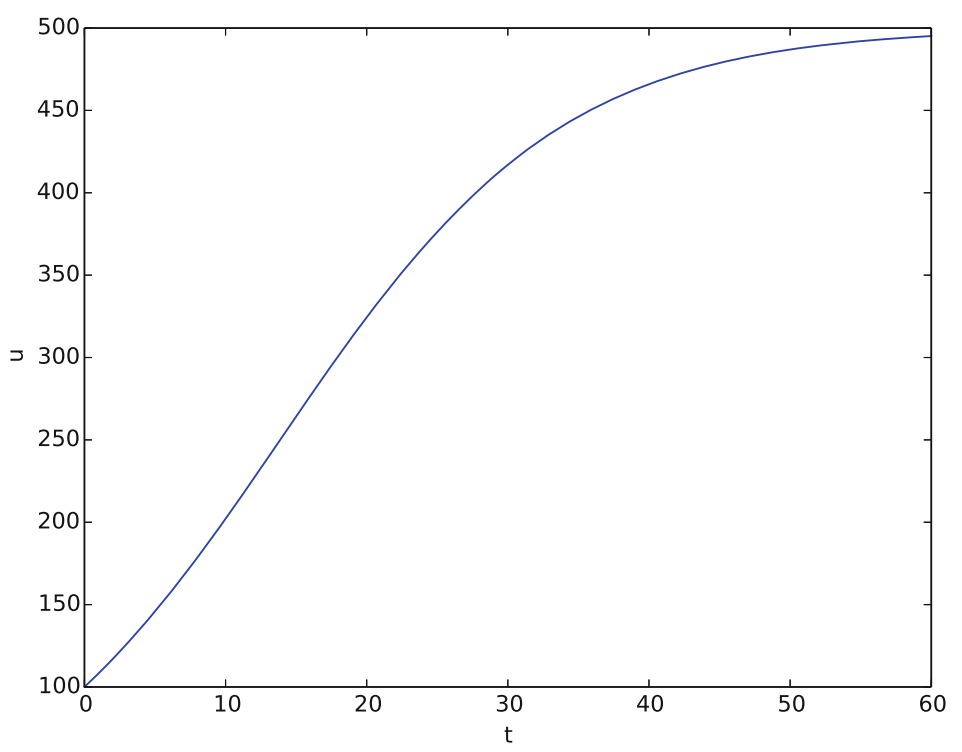

Fig. 4.7 Logistic growth of a population

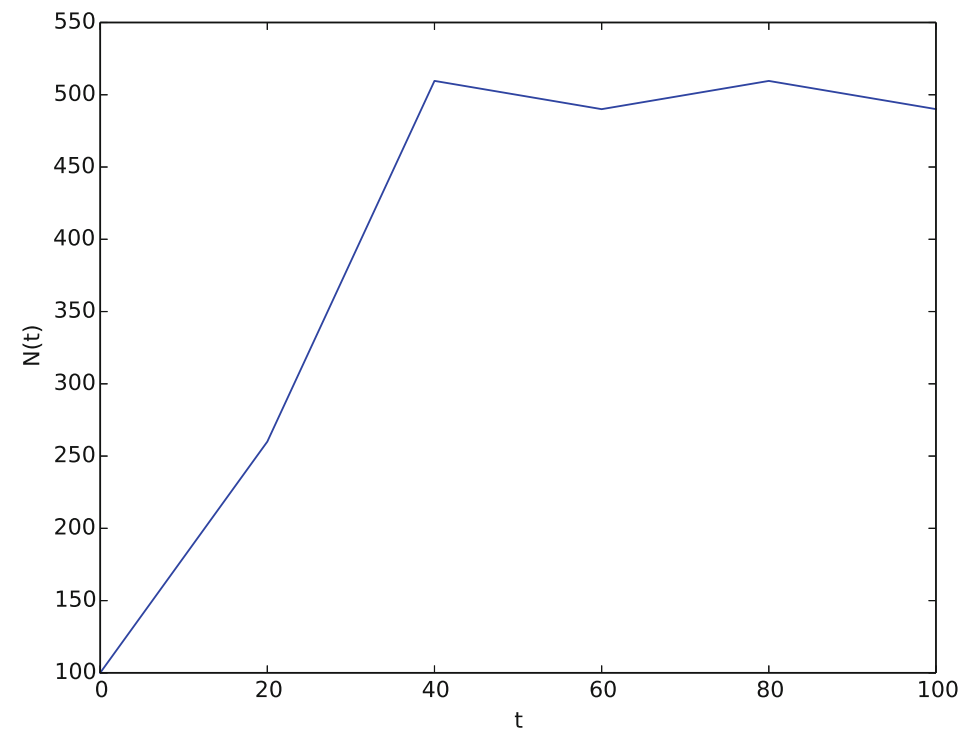

Fig. 4.8 Logistic growth with large time step

hence qualitatively wrong, because one can prove that the exact solution of the differential equation is monotone. (However, there is a corresponding difference equation model, $N_{n+1}=r N_{n}\left(1-N_{n} / M\right)$, which allows oscillatory solutions and those are observed in animal populations. The problem with large $\Delta t$ is that it just leads to wrong mathematics - and two wrongs don't make a right in terms of a relevant model.) 


\section{Remark on the world population}

The number of people on the planet ${ }^{2}$ follows the model $N^{\prime}=r(t) N$, where the net reproduction $r(t)$ varies with time and has decreased since its top in 1990. The current world value of $r$ is $1.2 \%$, and it is difficult to predict future values ${ }^{3}$. At the moment, the predictions of the world population point to a growth to 9.6 billion before declining.

This example shows the limitation of a differential equation model: we need to know all input parameters, including $r(t)$, in order to predict the future. It is seldom the case that we know all input parameters. Sometimes knowledge of the solution from measurements can help estimate missing input parameters.

\subsubsection{Verification: Exact Linear Solution of the Discrete Equations}

How can we verify that the programming of an ODE model is correct? The best method is to find a problem where there are no unknown numerical approximation errors, because we can then compare the exact solution of the problem with the result produced by our implementation and expect the difference to be within a very small tolerance. We shall base a unit test on this idea and implement a corresponding test function (see Sect. 3.4.4) for automatic verification of our implementation.

It appears that most numerical methods for ODEs will exactly reproduce a solution $u$ that is linear in $t$. We may therefore set $u=a t+b$ and choose any $f$ whose derivative is $a$. The choice $f(u, t)=a$ is very simple, but we may add anything that is zero, e.g.,

$$
f(u, t)=a+(u-(a t+b))^{m} .
$$

This is a valid $f(u, t)$ for any $a, b$, and $m$. The corresponding ODE looks highly non-trivial, however:

$$
u^{\prime}=a+(u-(a t+b))^{m} .
$$

Using the ode_FE function, we may write a proper test function as follows (in file test_ode_FE_exact_linear.m):

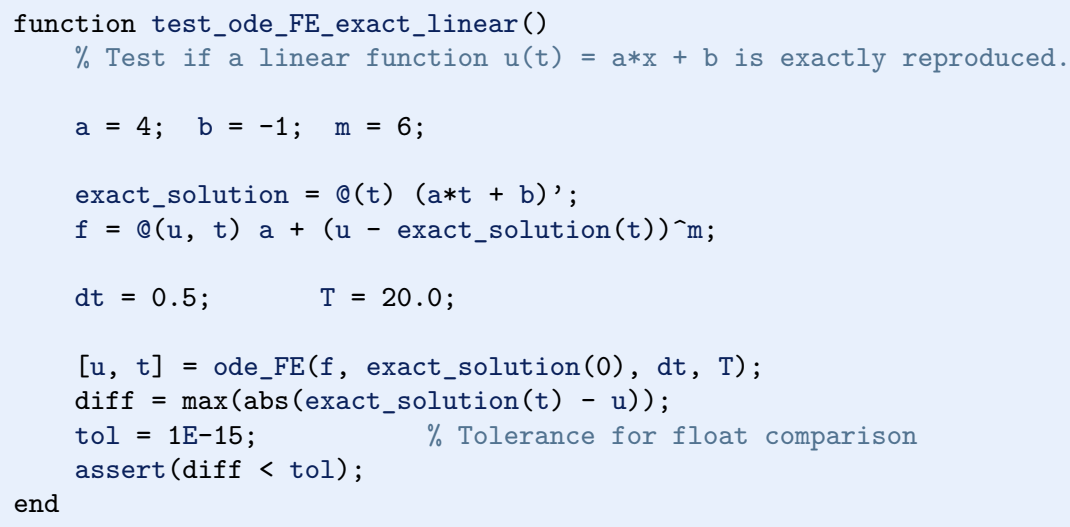

${ }^{2} \mathrm{http} / / /$ en.wikipedia.org/wiki/Population_growth

${ }^{3}$ http://users.rcn.com/jkimball.ma.ultranet/BiologyPages/P/Populations.html 
Observe that we cannot compare diff to zero, which is what we mathematically expect, because diff is a floating-point variable that most likely contains small rounding errors. Therefore, we must compare diff to zero with a tolerance, here $10^{-15}$.

You are encouraged to do Exercise 4.2 where the goal is to make a test function for a verification based on comparison with hand-calculated results for a few time steps.

\subsection{Spreading of Diseases}

Our aim with this section is to show in detail how one can apply mathematics and programming to investigate spreading of diseases. The mathematical model is now a system of three differential equations with three unknown functions. To derive such a model, we can use mainly intuition, so no specific background knowledge of diseases is required.

\subsubsection{Spreading of a Flu}

Imagine a boarding school out in the country side. This school is a small and closed society. Suddenly, one or more of the pupils get a flu. We expect that the flu may spread quite effectively or die out. The question is how many of the pupils and the school's staff will be affected. Some quite simple mathematics can help us to achieve insight into the dynamics of how the disease spreads.

Let the mathematical function $S(t)$ count how many individuals, at time $t$, that have the possibility to get infected. Here, $t$ may count hours or days, for instance. These individuals make up a category called susceptibles, labeled as S. Another category, I, consists of the individuals that are infected. Let $I(t)$ count how many there are in category I at time $t$. An individual having recovered from the disease is assumed to gain immunity. There is also a small possibility that an infected will die. In either case, the individual is moved from the I category to a category we call the removed category, labeled with $\mathrm{R}$. We let $R(t)$ count the number of individuals in the $R$ category at time $t$. Those who enter the $R$ category, cannot leave this category.

To summarize, the spreading of this disease is essentially the dynamics of moving individuals from the $\mathrm{S}$ to the I and then to the R category:

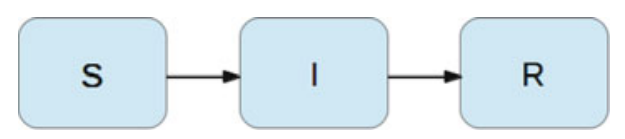

We can use mathematics to more precisely describe the exchange between the categories. The fundamental idea is to describe the changes that take place during a small time interval, denoted by $\Delta t$.

Our disease model is often referred to as a compartment model, where quantities are shuffled between compartments (here a synonym for categories) according to some rules. The rules express changes in a small time interval $\Delta t$, and from these 
changes we can let $\Delta t$ go to zero and obtain derivatives. The resulting equations then go from difference equations (with finite $\Delta t$ ) to differential equations ( $\Delta t \rightarrow$ $0)$.

We introduce a uniform mesh in time, $t_{n}=n \Delta t, n=0, \ldots, N_{t}$, and seek $S$ at the mesh points. The numerical approximation to $S$ at time $t_{n}$ is denoted by $S^{n}$. Similarly, we seek the unknown values of $I(t)$ and $R(t)$ at the mesh points and introduce a similar notation $I^{n}$ and $R^{n}$ for the approximations to the exact values $I\left(t_{n}\right)$ and $R\left(t_{n}\right)$.

In the time interval $\Delta t$ we know that some people will be infected, so $S$ will decrease. We shall soon argue by mathematics that there will be $\beta \Delta t S I$ new infected individuals in this time interval, where $\beta$ is a parameter reflecting how easy people get infected during a time interval of unit length. If the loss in $S$ is $\beta \Delta t S I$, we have that the change in $S$ is

$$
S^{n+1}-S^{n}=-\beta \Delta t S^{n} I^{n} .
$$

Dividing by $\Delta t$ and letting $\Delta t \rightarrow 0$, makes the left-hand side approach $S^{\prime}\left(t_{n}\right)$ such that we obtain a differential equation

$$
S^{\prime}=-\beta S I
$$

The reasoning in going from the difference equation (4.9) to the differential equation (4.10) follows exactly the steps explained in Sect. 4.1.1.

Before proceeding with how $I$ and $R$ develops in time, let us explain the formula $\beta \Delta t S I$. We have $S$ susceptibles and $I$ infected people. These can make up $S I$ pairs. Now, suppose that during a time interval $T$ we measure that $m$ actual pairwise meetings do occur among $n$ theoretically possible pairings of people from the $S$ and I categories. The probability that people meet in pairs during a time $T$ is (by the empirical frequency definition of probability) equal to $m / n$, i.e., the number of successes divided by the number of possible outcomes. From such statistics we normally derive quantities expressed per unit time, i.e., here we want the probability per unit time, $\mu$, which is found from dividing by $T: \mu=m /(n T)$.

Given the probability $\mu$, the expected number of meetings per time interval of $S I$ possible pairs of people is (from basic statistics) $\mu S I$. During a time interval $\Delta t$, there will be $\mu S I \Delta t$ expected number of meetings between susceptibles and infected people such that the virus may spread. Only a fraction of the $\mu \Delta t S I$ meetings are effective in the sense that the susceptible actually becomes infected. Counting that $m$ people get infected in $n$ such pairwise meetings (say 5 are infected from 1000 meetings), we can estimate the probability of being infected as $p=$ $m / n$. The expected number of individuals in the $\mathrm{S}$ category that in a time interval $\Delta t$ catch the virus and get infected is then $p \mu \Delta t S I$. Introducing a new constant $\beta=p \mu$ to save some writing, we arrive at the formula $\beta \Delta t S I$.

The value of $\beta$ must be known in order to predict the future with the disease model. One possibility is to estimate $p$ and $\mu$ from their meanings in the derivation above. Alternatively, we can observe an "experiment" where there are $S_{0}$ susceptibles and $I_{0}$ infected at some point in time. During a time interval $T$ we count that $N$ susceptibles have become infected. Using (4.9) as a rough approximation of how $S$ has developed during time $T$ (and now $T$ is not necessarily small, but we use (4.9) 
anyway), we get

$$
N=\beta T S_{0} I_{0} \Rightarrow \beta=\frac{N}{T S_{0} I_{0}} .
$$

We need an additional equation to describe the evolution of $I(t)$. Such an equation is easy to establish by noting that the loss in the $\mathrm{S}$ category is a corresponding gain in the I category. More precisely,

$$
I^{n+1}-I^{n}=\beta \Delta t S^{n} I^{n} .
$$

However, there is also a loss in the I category because people recover from the disease. Suppose that we can measure that $m$ out of $n$ individuals recover in a time period $T$ (say 10 of 40 sick people recover during a day: $m=10, n=40, T=$ $24 \mathrm{~h})$. Now, $\gamma=m /(n T)$ is the probability that one individual recovers in a unit time interval. Then (on average) $\gamma \Delta t I$ infected will recover in a time interval $\Delta t$. This quantity represents a loss in the I category and a gain in the R category. We can therefore write the total change in the I category as

$$
I^{n+1}-I^{n}=\beta \Delta t S^{n} I^{n}-\gamma \Delta t I^{n} .
$$

The change in the $\mathrm{R}$ category is simple: there is always an increase from the I category:

$$
R^{n+1}-R^{n}=\gamma \Delta t I^{n} .
$$

Since there is no loss in the R category (people are either recovered and immune, or dead), we are done with the modeling of this category. In fact, we do not strictly need the equation (4.14) for $R$, but extensions of the model later will need an equation for $R$.

Dividing by $\Delta t$ in (4.13) and (4.14) and letting $\Delta t \rightarrow 0$, results in the corresponding differential equations

$$
I^{\prime}=\beta S I-\gamma I
$$

and

$$
R^{\prime}=\gamma I
$$

To summarize, we have derived difference equations (4.9)-(4.14), and alternative differential equations (4.15)-(4.16). For reference, we list the complete set of the three difference equations:

$$
\begin{aligned}
& S^{n+1}=S^{n}-\beta \Delta t S^{n} I^{n}, \\
& I^{n+1}=I^{n}+\beta \Delta t S^{n} I^{n}-\gamma \Delta t I^{n}, \\
& R^{n+1}=R^{n}+\gamma \Delta t I^{n} .
\end{aligned}
$$

Note that we have isolated the new unknown quantities $S^{n+1}, I^{n+1}$, and $R^{n+1}$ on the left-hand side, such that these can readily be computed if $S^{n}, I^{n}$, and $R^{n}$ are known. To get such a procedure started, we need to know $S^{0}, I^{0}, R^{0}$. Obviously, we also need to have values for the parameters $\beta$ and $\gamma$. 
We also list the system of three differential equations:

$$
\begin{aligned}
S^{\prime} & =-\beta S I, \\
I^{\prime} & =\beta S I-\gamma I, \\
R^{\prime} & =\gamma I .
\end{aligned}
$$

This differential equation model (and also its discrete counterpart above) is known as an SIR model. The input data to the differential equation model consist of the parameters $\beta$ and $\gamma$ as well as the initial conditions $S(0)=S_{0}, I(0)=I_{0}$, and $R(0)=R_{0}$.

\subsubsection{A Forward Euler Method for the Differential Equation System}

Let us apply the same principles as we did in Sect. 4.1.2 to discretize the differential equation system by the Forward Euler method. We already have a time mesh and time-discrete quantities $S^{n}, I^{n}, R^{n}, n=0, \ldots, N_{t}$. The three differential equations are assumed to be valid at the mesh points. At the point $t_{n}$ we then have

$$
\begin{aligned}
S^{\prime}\left(t_{n}\right) & =-\beta S\left(t_{n}\right) I\left(t_{n}\right), \\
I^{\prime}\left(t_{n}\right) & =\beta S\left(t_{n}\right) I\left(t_{n}\right)-\gamma I\left(t_{n}\right), \\
R^{\prime}\left(t_{n}\right) & =\gamma I\left(t_{n}\right),
\end{aligned}
$$

for $n=0,1, \ldots, N_{t}$. This is an approximation since the differential equations are originally valid at all times $t$ (usually in some finite interval $[0, T]$ ). Using forward finite differences for the derivatives results in an additional approximation,

$$
\begin{aligned}
& \frac{S^{n+1}-S^{n}}{\Delta t}=-\beta S^{n} I^{n}, \\
& \frac{I^{n+1}-I^{n}}{\Delta t}=\beta S^{n} I^{n}-\gamma I^{n}, \\
& \frac{R^{n+1}-R^{n}}{\Delta t}=\gamma I^{n} .
\end{aligned}
$$

As we see, these equations are identical to the difference equations that naturally arise in the derivation of the model. However, other numerical methods than the Forward Euler scheme will result in slightly different difference equations.

\subsubsection{Programming the Numerical Method; the Special Case}

The computation of (4.26)-(4.28) can be readily made in a computer program SIR1.m:

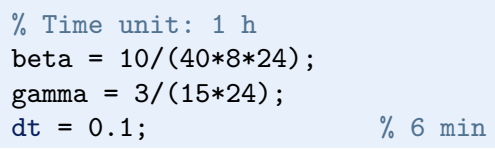




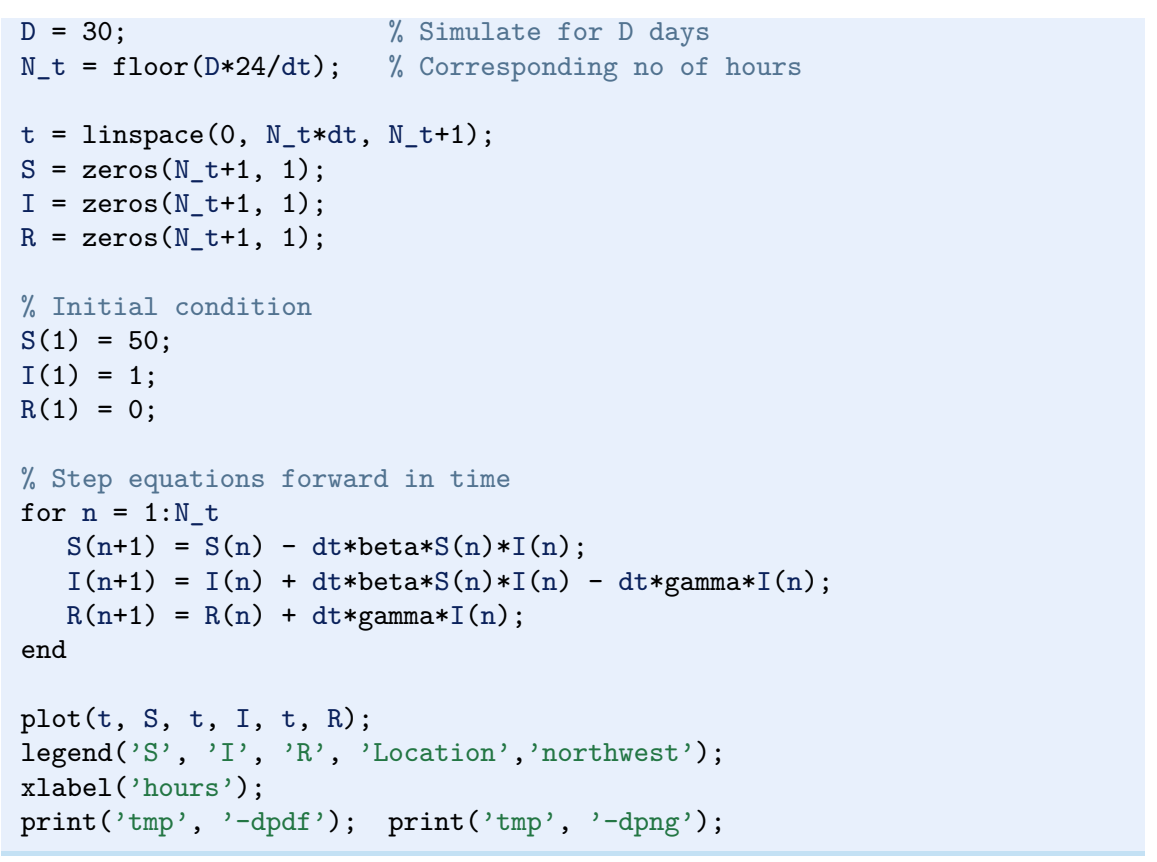

This program was written to investigate the spreading of a flu at the mentioned boarding school, and the reasoning for the specific choices $\beta$ and $\gamma$ goes as follows. At some other school where the disease has already spread, it was observed that in the beginning of a day there were 40 susceptibles and 8 infected, while the numbers were 30 and 18, respectively, 24 hours later. Using $1 \mathrm{~h}$ as time unit, we then have from (4.11) that $\beta=10 /(40 \cdot 8 \cdot 24)$. Among 15 infected, it was observed that 3 recovered during a day, giving $\gamma=3 /(15 \cdot 24)$. Applying these parameters to a new case where there is one infected initially and 50 susceptibles, gives the graphs in Fig. 4.9. These graphs are just straight lines between the values at times $t_{i}=i \Delta t$ as computed by the program. We observe that $S$ reduces as $I$ and $R$ grows. After about 30 days everyone has become ill and recovered again.

We can experiment with $\beta$ and $\gamma$ to see whether we get an outbreak of the disease or not. Imagine that a "wash your hands" campaign was successful and that the other school in this case experienced a reduction of $\beta$ by a factor of 5. With this lower $\beta$ the disease spreads very slowly so we simulate for 60 days. The curves appear in Fig. 4.10.

\subsubsection{Outbreak or Not}

Looking at the equation for $I$, it is clear that we must have $\beta S I-\gamma I>0$ for $I$ to increase. When we start the simulation it means that

$$
\beta S(0) I(0)-\gamma I(0)>0,
$$

or simpler

$$
\frac{\beta S(0)}{\gamma}>1
$$




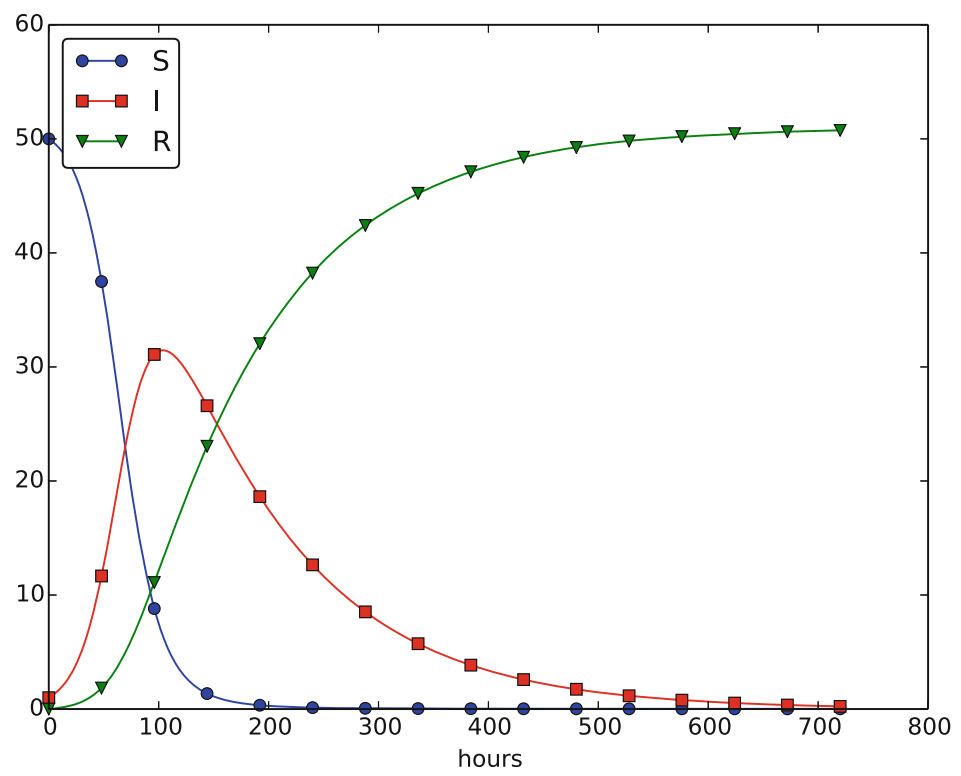

Fig. 4.9 Natural evolution of a flu at a boarding school

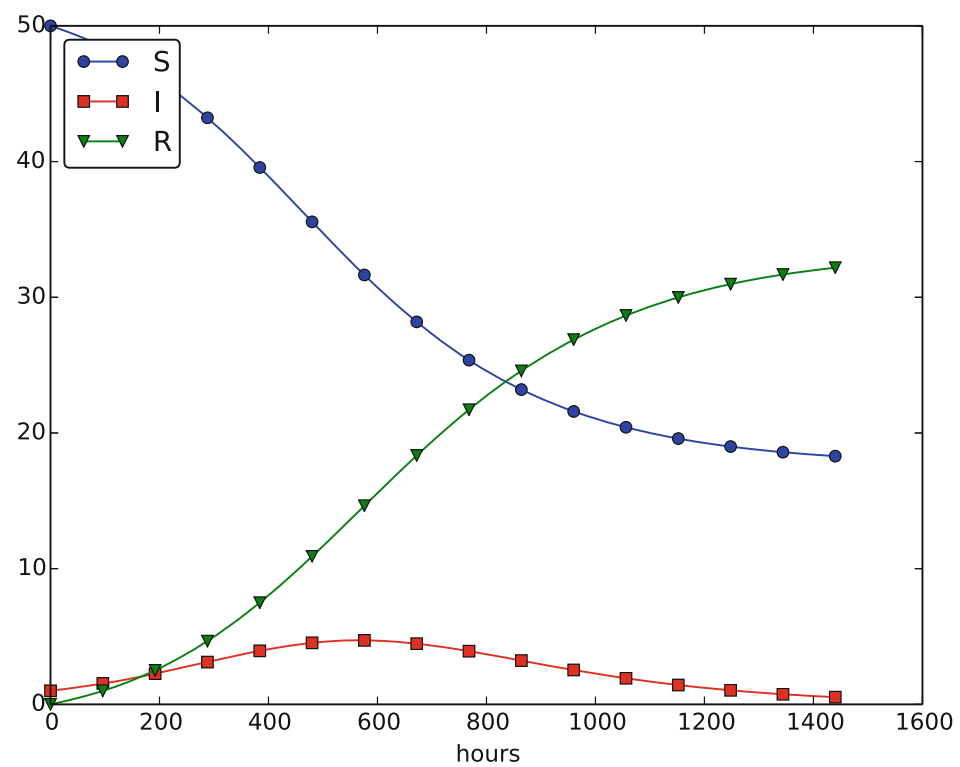

Fig. 4.10 Small outbreak of a flu at a boarding school ( $\beta$ is much smaller than in Fig. 4.9)

to increase the number of infected people and accelerate the spreading of the disease. You can run the SIR1.m program with a smaller $\beta$ such that (4.29) is violated and observe that there is no outbreak. 


\section{The power of mathematical modeling}

The reader should notice our careful use of words in the previous paragraphs. We started out with modeling a very specific case, namely the spreading of a flu among pupils and staff at a boarding school. With purpose we exchanged words like pupils and flu with more neutral and general words like individuals and disease, respectively. Phrased equivalently, we raised the abstraction level by moving from a specific case (flu at a boarding school) to a more general case (disease in a closed society). Very often, when developing mathematical models, we start with a specific example and see, through the modeling, that what is going on of essence in this example also will take place in many similar problem settings. We try to incorporate this generalization in the model so that the model has a much wider application area than what we aimed at in the beginning. This is the very power of mathematical modeling: by solving one specific case we have often developed more generic tools that can readily be applied to solve seemingly different problems. The next sections will give substance to this assertion.

\subsubsection{Abstract Problem and Notation}

When we had a specific differential equation with one unknown, we quickly turned to an abstract differential equation written in the generic form $u^{\prime}=f(u, t)$. We refer to such a problem as a scalar $O D E$. A specific equation corresponds to a specific choice of the formula $f(u, t)$ involving $u$ and (optionally) $t$.

It is advantageous to also write a system of differential equations in the same abstract notation,

$$
u^{\prime}=f(u, t),
$$

but this time it is understood that $u$ is a vector of functions and $f$ is also vector. We say that $u^{\prime}=f(u, t)$ is a vector ODE or system of ODEs in this case. For the SIR model we introduce the two 3-vectors, one for the unknowns,

$$
u=(S(t), I(t), R(t)),
$$

and one for the right-hand side functions,

$$
f(u, t)=(-\beta S I, \beta S I-\gamma I, \gamma I) .
$$

The equation $u^{\prime}=f(u, t)$ means setting the two vectors equal, i.e., the components must be pairwise equal. Since $u^{\prime}=\left(S^{\prime}, I^{\prime}, R^{\prime}\right)$, we get that $u^{\prime}=f$ implies

$$
\begin{aligned}
S^{\prime} & =-\beta S I, \\
I^{\prime} & =\beta S I-\gamma I, \\
R^{\prime} & =\gamma I .
\end{aligned}
$$

The generalized short notation $u^{\prime}=f(u, t)$ is very handy since we can derive numerical methods and implement software for this abstract system and in a particular application just identify the formulas in the $f$ vector, implement these, and call functionality that solves the differential equation system. 


\subsubsection{Programming the Numerical Method; the General Case}

In Matlab code, the Forward Euler step

$$
u^{n+1}=u^{n}+\Delta t f\left(u^{n}, t_{n}\right),
$$

being a scalar or a vector equation, can be coded as

$$
u(n+1,:)=u(n,:)+d t * f(u(n,:), t(n))
$$

both in the scalar and vector case. In the vector case, $\mathrm{u}(\mathrm{n},:)$ is a one-dimensional array of length $m+1$ holding the mathematical quantity $u^{n}$, and the Matlab function $f$ must return an array of length $m+1$. Then the expression $u(n,:)+$ $d t * f(u(n,:), t(n))$ is an array plus a scalar times an array.

For all this to work, the complete numerical solution must be represented by a two-dimensional array, created by $u=\operatorname{zeros}\left(N_{-} t+1, m+1\right)$. The first index counts the time points and the second the components of the solution vector at one time point. That is, $\mathrm{u}(\mathrm{n}, \mathrm{i})$ corresponds to the mathematical quantity $u_{i}^{n}$. Writing $\mathrm{u}(\mathrm{n}, \mathrm{:})$ picks out all the components in the solution at the time point with index $\mathrm{n}$. The nice feature of these facts is that the same piece of Matlab code works for both a scalar ODE and a system of ODEs!

The ode_FE function for the vector ODE is placed in the file ode_FE.m and was written as follows:

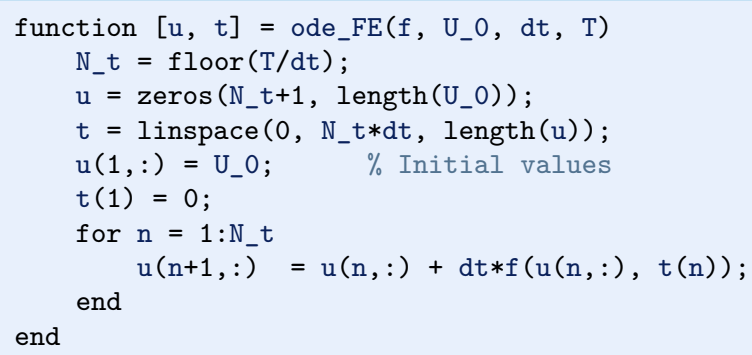

Let us show how the previous SIR model can be solved using the new general ode_FE that can solve any vector ODE. The user's $f(u, t)$ function takes a vector u, with three components corresponding to $S, I$, and $R$ as argument, along with the current time point $t(n)$, and must return the values of the formulas of the right-hand sides in the vector ODE. An appropriate implementation is

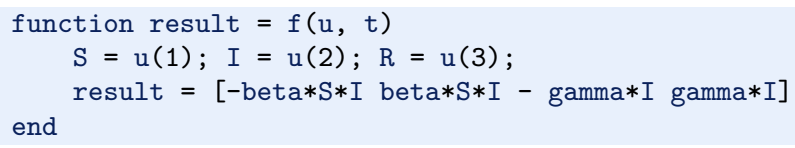

where beta and gamma are problem specific parameters set outside of that function. Note that the S, I, and $\mathrm{R}$ values correspond to $S^{n}, I^{n}$, and $R^{n}$. These values are then just inserted in the various formulas in the vector ODE. 
We can now show a function (in file demo_SIR.m) that runs the previous SIR example, but which applies the generic ode_FE function:

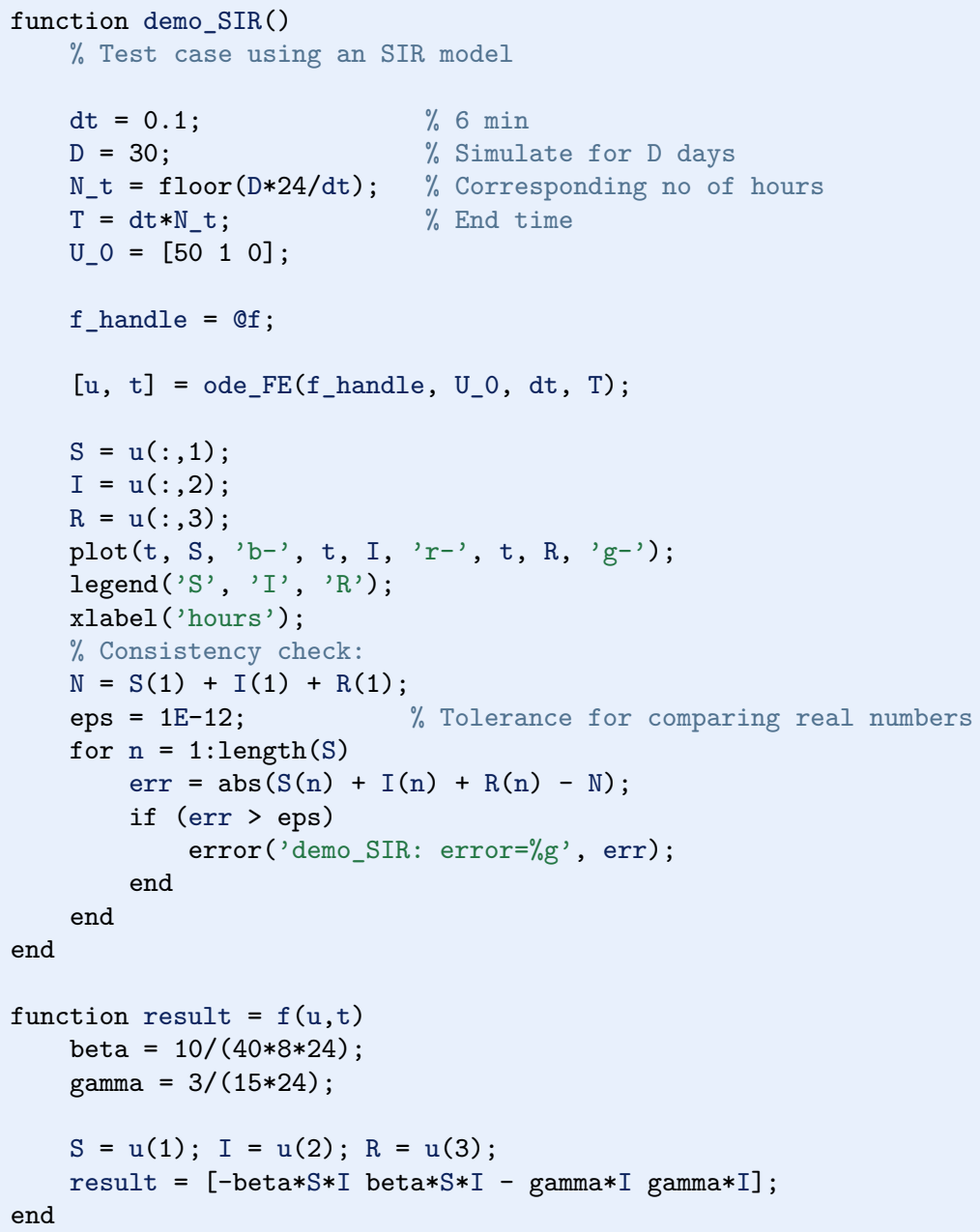

Recall that the $\mathrm{u}$ returned from ode_FE contains all components $(S, I, R)$ in the solution vector at all time points. We therefore need to extract the $S, I$, and $R$ values in separate arrays for further analysis and easy plotting.

Another key feature of this higher-quality code is the consistency check. By adding the three differential equations in the SIR model, we realize that $S^{\prime}+I^{\prime}+$ $R^{\prime}=0$, which means that $S+I+R=$ const. We can check that this relation holds by comparing $S^{n}+I^{n}+R^{n}$ to the sum of the initial conditions. The check is not a full-fledged verification, but it is a much better than doing nothing and hoping that the computation is correct. Exercise 4.5 suggests another method for controlling the quality of the numerical solution. 


\subsubsection{Time-Restricted Immunity}

Let us now assume that immunity after the disease only lasts for some certain time period. This means that there is transport from the $\mathrm{R}$ state to the $\mathrm{S}$ state:

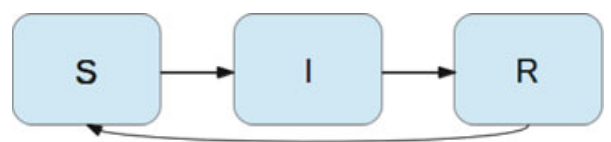

Modeling the loss of immunity is very similar to modeling recovery from the disease: the amount of people losing immunity is proportional to the amount of recovered patients and the length of the time interval $\Delta t$. We can therefore write the loss in the $\mathrm{R}$ category as $-v \Delta t R$ in time $\Delta t$, where $v^{-1}$ is the typical time it takes to lose immunity. The loss in $R(t)$ is a gain in $S(t)$. The "budgets" for the categories therefore become

$$
\begin{aligned}
& S^{n+1}=S^{n}-\beta \Delta t S^{n} I^{n}+v \Delta t R^{n}, \\
& I^{n+1}=I^{n}+\beta \Delta t S^{n} I^{n}-\gamma \Delta t I^{n}, \\
& R^{n+1}=R^{n}+\gamma \Delta t I^{n}-v \Delta t R^{n} .
\end{aligned}
$$

Dividing by $\Delta t$ and letting $\Delta t \rightarrow 0$ gives the differential equation system

$$
\begin{aligned}
& S^{\prime}=-\beta S I+v R, \\
& I^{\prime}=\beta S I-\gamma I, \\
& R^{\prime}=\gamma I-v R .
\end{aligned}
$$

This system can be solved by the same methods as we demonstrated for the original SIR model. Only one modification in the program is necessary: adding nu*R [n] to the $S[n+1]$ update and subtracting the same quantity in the $R[n+1]$ update:

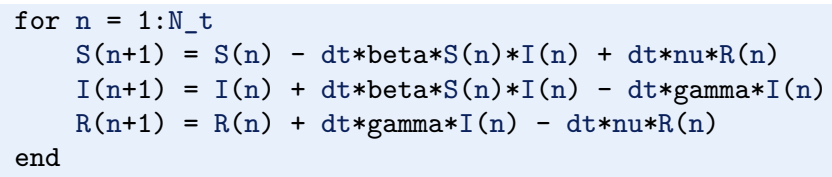

The modified code is found in the file SIR2.m.

Setting $v^{-1}$ to 50 days, reducing $\beta$ by a factor of 4 compared to the previous example $(\beta=0.00033)$, and simulating for 300 days gives an oscillatory behavior in the categories, as depicted in Fig. 4.11. It is easy now to play around and study how the parameters affect the spreading of the disease. For example, making the disease slightly more effective (increase $\beta$ to 0.00043 ) and increasing the average time to loss of immunity to 90 days lead to other oscillations, see Fig. 4.12.

\subsubsection{Incorporating Vaccination}

We can extend the model to also include vaccination. To this end, it can be useful to track those who are vaccinated and those who are not. So, we introduce a fourth 


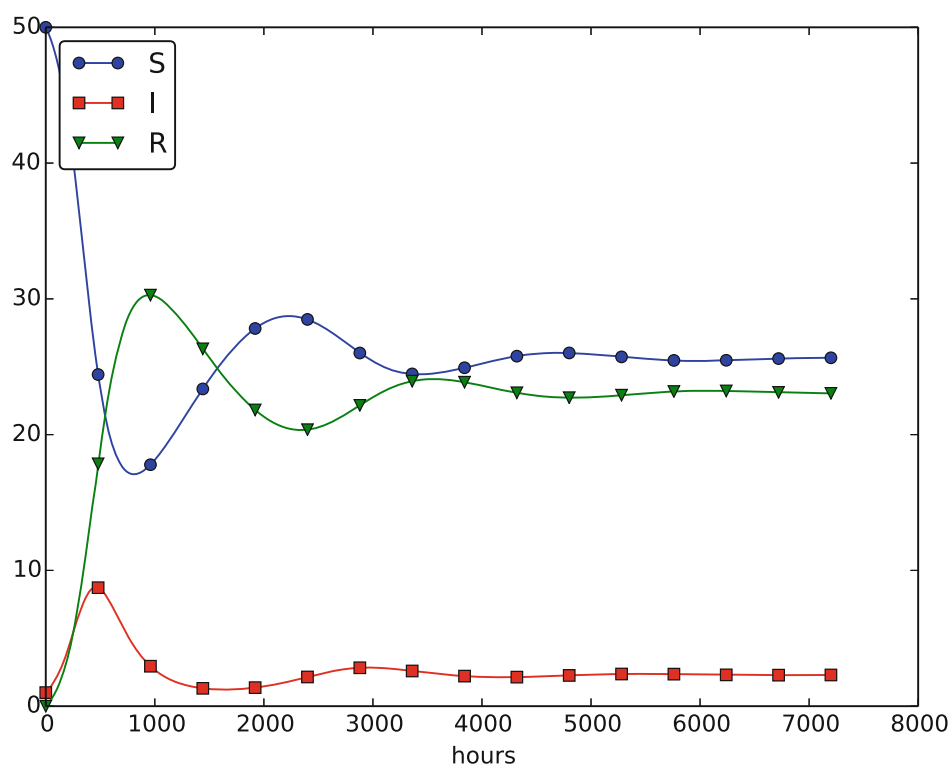

Fig. 4.11 Including loss of immunity

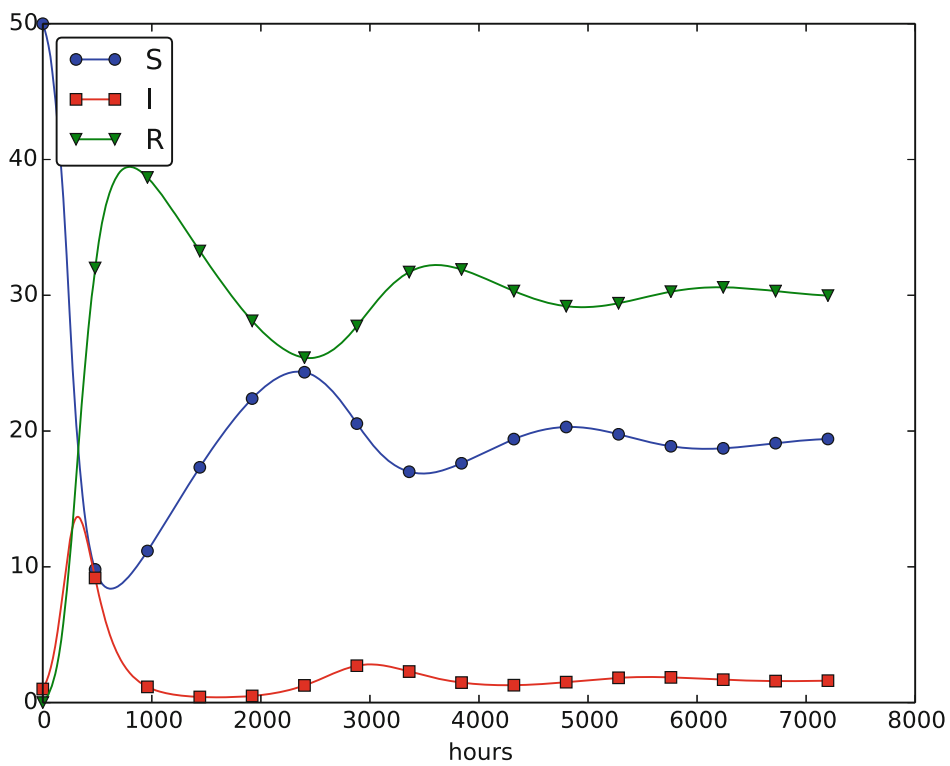

Fig. 4.12 Increasing $\beta$ and reducing $v$ compared to Fig. 4.11

category, V, for those who have taken a successful vaccination. Furthermore, we assume that in a time interval $\Delta t$, a fraction $p \Delta t$ of the $\mathrm{S}$ category is subject to a successful vaccination. This means that in the time $\Delta t, p \Delta t S$ people leave from the $\mathrm{S}$ to the $\mathrm{V}$ category. Since the vaccinated ones cannot get the disease, there is no 

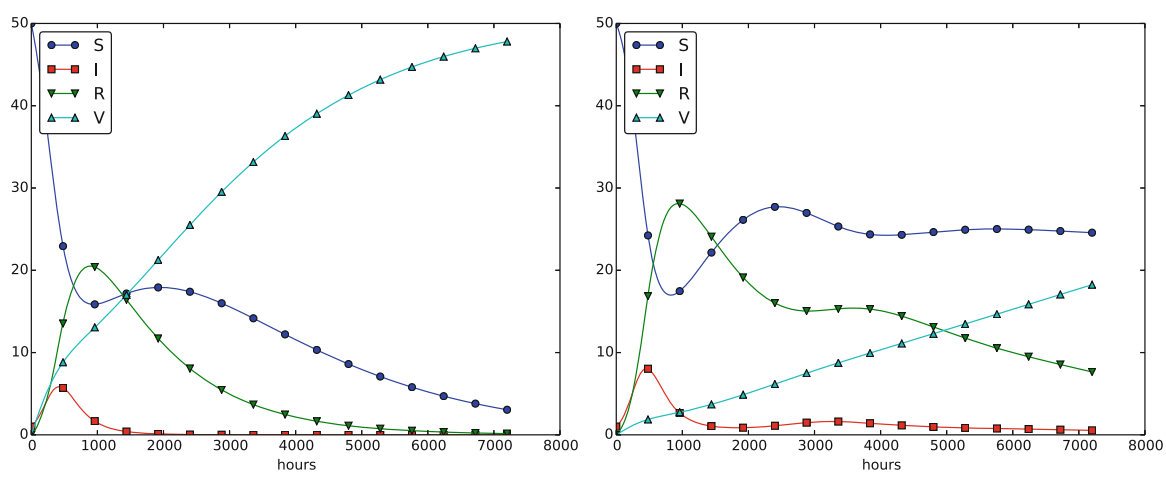

Fig. 4.13 The effect of vaccination: $p=0.0005$ (left) and $p=0.0001$ (right)

impact on the I or R categories. We can visualize the categories, and the movement between them, as

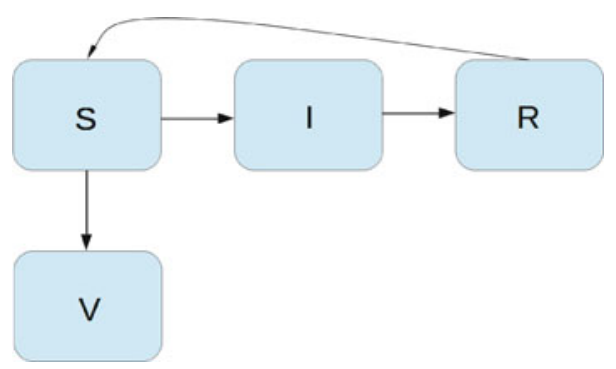

The new, extended differential equations with the $V$ quantity become

$$
\begin{aligned}
S^{\prime} & =-\beta S I+v R-p S, \\
V^{\prime} & =p S, \\
I^{\prime} & =\beta S I-\gamma I, \\
R^{\prime} & =\gamma I-v R .
\end{aligned}
$$

We shall refer to this model as the SIRV model.

The new equation for $V^{\prime}$ poses no difficulties when it comes to the numerical method. In a Forward Euler scheme we simply add an update

$$
V^{n+1}=V^{n}+p \Delta t S^{n} .
$$

The program needs to store $V(t)$ in an additional array $\mathrm{V}$, and the plotting command must be extended with more arguments to plot $\mathrm{V}$ versus $\mathrm{t}$ as well. The complete code is found in the file SIRV1.m.

Using $p=0.0005$ and $p=0.0001$ as values for the vaccine efficiency parameter, the effect of vaccination is seen in Fig. 4.13 (other parameters are as in Fig. 4.11). 


\subsubsection{Discontinuous Coefficients: a Vaccination Campaign}

What about modeling a vaccination campaign? Imagine that six days after the outbreak of the disease, the local health station launches a vaccination campaign. They reach out to many people, say 10 times as efficiently as in the previous (constant vaccination) case. If the campaign lasts for 10 days we can write

$$
p(t)= \begin{cases}0.005, & 6 \cdot 24 \leq t \leq 15 \cdot 24 \\ 0, & \text { otherwise }\end{cases}
$$

Note that we must multiply the $t$ value by 24 because $t$ is measured in hours, not days. In the differential equation system, $p S(t)$ must be replaced by $p(t) S(t)$, and in this case we get a differential equation system with a term that is discontinuous. This is usually quite a challenge in mathematics, but as long as we solve the equations numerically in a program, a discontinuous coefficient is easy to treat.

There are two ways to implement the discontinuous coefficient $p(t)$ : through a function and through an array. The function approach is perhaps the easiest:

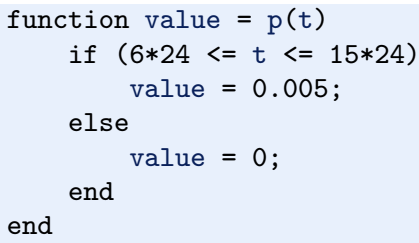

In the code for updating the arrays $\mathrm{S}$ and $\mathrm{V}$ we get a term $\mathrm{p}(\mathrm{t}(\mathrm{n})) * \mathrm{~S}(\mathrm{n})$.

We can also let $p(t)$ be an array filled with correct values prior to the simulation. Then we need to allocate an array $p$ of length $N_{-} t+1$ and find the indices corresponding to the time period between 6 and 15 days. These indices are found from the time point divided by $\Delta t$. That is,

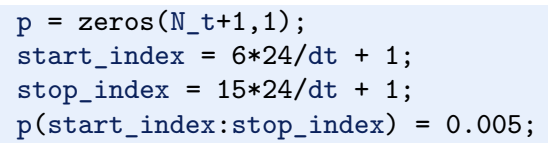

The $p(t) S(t)$ term in the updating formulas for $S$ and $V$ simply becomes $\mathrm{p}(\mathrm{n}) *$ $\mathrm{S}(\mathrm{n})$. The file SIRV2. $\mathrm{m}$ contains a program based on filling an array $\mathrm{p}$.

The effect of a vaccination campaign is illustrated in Fig. 4.14. All the data are as in Fig. 4.13 (left), except that $p$ is ten times stronger for a period of 10 days and $p=0$ elsewhere. 


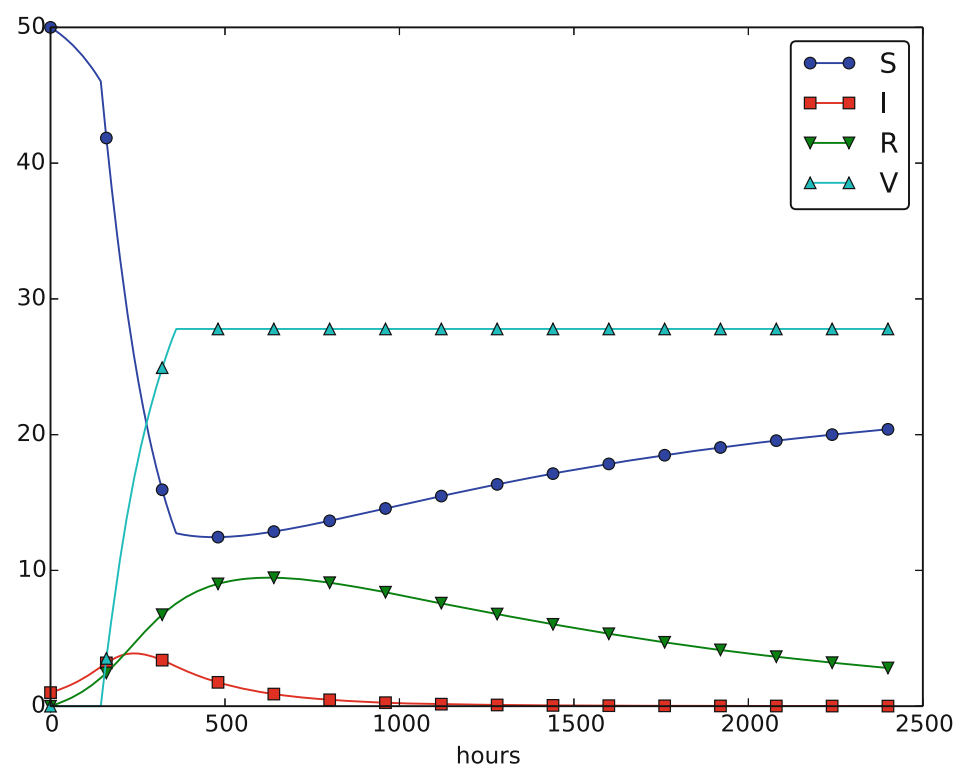

Fig. 4.14 The effect of a vaccination campaign

\subsection{Oscillating One-Dimensional Systems}

Numerous engineering constructions and devices contain materials that act like springs. Such springs give rise to oscillations, and controlling oscillations is a key engineering task. We shall now learn to simulate oscillating systems.

As always, we start with the simplest meaningful mathematical model, which for oscillations is a second-order differential equation:

$$
u^{\prime \prime}(t)+\omega^{2} u(t)=0,
$$

where $\omega$ is a given physical parameter. Equation (4.40) models a one-dimensional system oscillating without damping (i.e., with negligible damping). One-dimensional here means that some motion takes place along one dimension only in some coordinate system. Along with (4.40) we need the two initial conditions $u(0)$ and $u^{\prime}(0)$.

\subsubsection{Derivation of a Simple Model}

Many engineering systems undergo oscillations, and differential equations constitute the key tool to understand, predict, and control the oscillations. We start with the simplest possible model that captures the essential dynamics of an oscillating system. Some body with mass $m$ is attached to a spring and moves along a line without friction, see Fig. 4.15 for a sketch (rolling wheels indicate "no friction"). When the spring is stretched (or compressed), the spring force pulls (or pushes) the body back and work "against" the motion. More precisely, let $x(t)$ be the position 


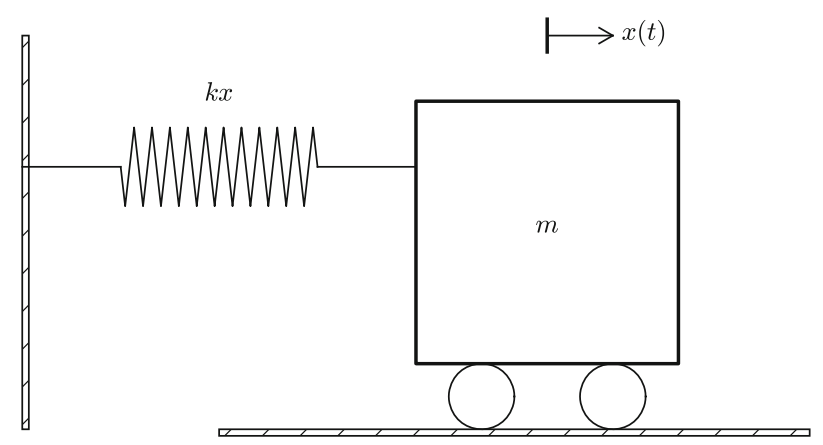

Fig. 4.15 Sketch of a one-dimensional, oscillating dynamic system (without friction)

of the body on the $x$ axis, along which the body moves. The spring is not stretched when $x=0$, so the force is zero, and $x=0$ is hence the equilibrium position of the body. The spring force is $-k x$, where $k$ is a constant to be measured. We assume that there are no other forces (e.g., no friction). Newton's 2nd law of motion $F=m a$ then has $F=-k x$ and $a=\ddot{x}$,

$$
-k x=m \ddot{x}
$$

which can be rewritten as

$$
\ddot{x}+\omega^{2} x=0,
$$

by introducing $\omega=\sqrt{\mathrm{k} / \mathrm{m}}$ (which is very common).

Equation (4.42) is a second-order differential equation, and therefore we need two initial conditions, one on the position $x(0)$ and one on the velocity $x^{\prime}(0)$. Here we choose the body to be at rest, but moved away from its equilibrium position:

$$
x(0)=X_{0}, \quad x^{\prime}(0)=0 .
$$

The exact solution of (4.42) with these initial conditions is $x(t)=X_{0} \cos \omega t$. This can easily be verified by substituting into (4.42) and checking the initial conditions. The solution tells that such a spring-mass system oscillates back and forth as described by a cosine curve.

The differential equation (4.42) appears in numerous other contexts. A classical example is a simple pendulum that oscillates back and forth. Physics books derive, from Newton's second law of motion, that

$$
m L \theta^{\prime \prime}+m g \sin \theta=0
$$

where $m$ is the mass of the body at the end of a pendulum with length $L, g$ is the acceleration of gravity, and $\theta$ is the angle the pendulum makes with the vertical. Considering small angles $\theta, \sin \theta \approx \theta$, and we get (4.42) with $x=\theta, \omega=\sqrt{g / L}$, $x(0)=\Theta$, and $x^{\prime}(0)=0$, if $\Theta$ is the initial angle and the pendulum is at rest at $t=0$. 


\subsubsection{Numerical Solution}

We have not looked at numerical methods for handling second-order derivatives, and such methods are an option, but we know how to solve first-order differential equations and even systems of first-order equations. With a little, yet very common, trick we can rewrite (4.42) as a first-order system of two differential equations. We introduce $u=x$ and $v=x^{\prime}=u^{\prime}$ as two new unknown functions. The two corresponding equations arise from the definition $v=u^{\prime}$ and the original equation (4.42):

$$
\begin{aligned}
& u^{\prime}=v, \\
& v^{\prime}=-\omega^{2} u .
\end{aligned}
$$

(Notice that we can use $u^{\prime \prime}=v^{\prime}$ to remove the second-order derivative from Newton's 2nd law.)

We can now apply the Forward Euler method to (4.43)-(4.44), exactly as we did in Sect. 4.2.2:

$$
\begin{aligned}
& \frac{u^{n+1}-u^{n}}{\Delta t}=v^{n}, \\
& \frac{v^{n+1}-v^{n}}{\Delta t}=-\omega^{2} u^{n},
\end{aligned}
$$

resulting in the computational scheme

$$
\begin{aligned}
& u^{n+1}=u^{n}+\Delta t v^{n}, \\
& v^{n+1}=v^{n}-\Delta t \omega^{2} u^{n} .
\end{aligned}
$$

\subsubsection{Programming the Numerical Method; the Special Case}

A simple program for (4.47)-(4.48) follows the same ideas as in Sect. 4.2.3:

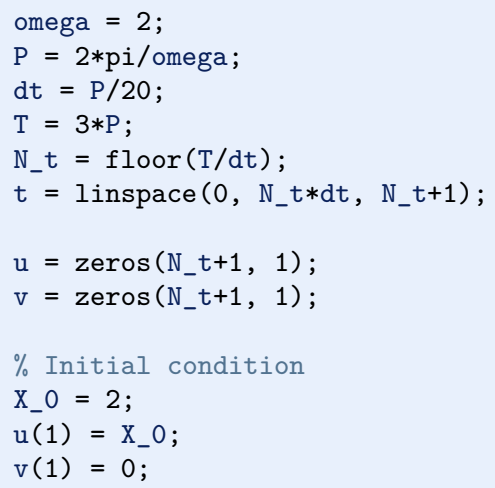




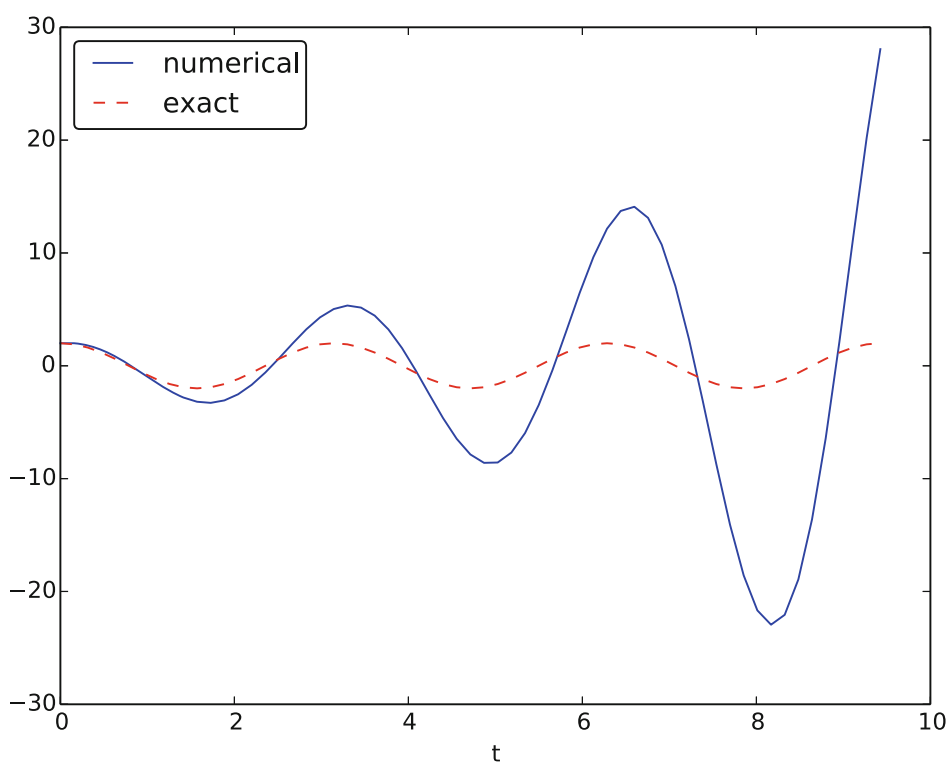

Fig.4.16 Simulation of an oscillating system

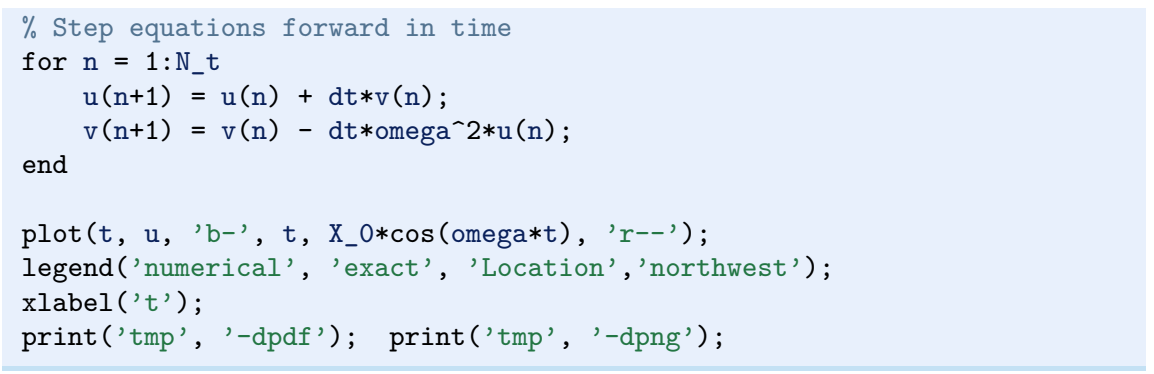

(See file osc_FE_special_case.m.)

Since we already know the exact solution as $u(t)=X_{0} \cos \omega t$, we have reasoned as follows to find an appropriate simulation interval $[0, T]$ and also how many points we should choose. The solution has a period $P=2 \pi / \omega$. (The period $P$ is the time difference between two peaks of the $u(t) \sim \cos \omega t$ curve.) Simulating for three periods of the cosine function, $T=3 P$, and choosing $\Delta t$ such that there are 20 intervals per period gives $\Delta t=P / 20$ and a total of $N_{t}=T / \Delta t$ intervals. The rest of the program is a straightforward coding of the Forward Euler scheme.

Figure 4.16 shows a comparison between the numerical solution and the exact solution of the differential equation. To our surprise, the numerical solution looks wrong. Is this discrepancy due to a programming error or a problem with the Forward Euler method?

First of all, even before trying to run the program, you should sit down and compute two steps in the time loop with a calculator so you have some intermediate results to compare with. Using $X_{0}=2, d t=0.157079632679$, and $\omega=2$, we get $u^{1}=2, v^{1}=-1.25663706, u^{2}=1.80260791$, and $v^{2}=-2.51327412$. Such 

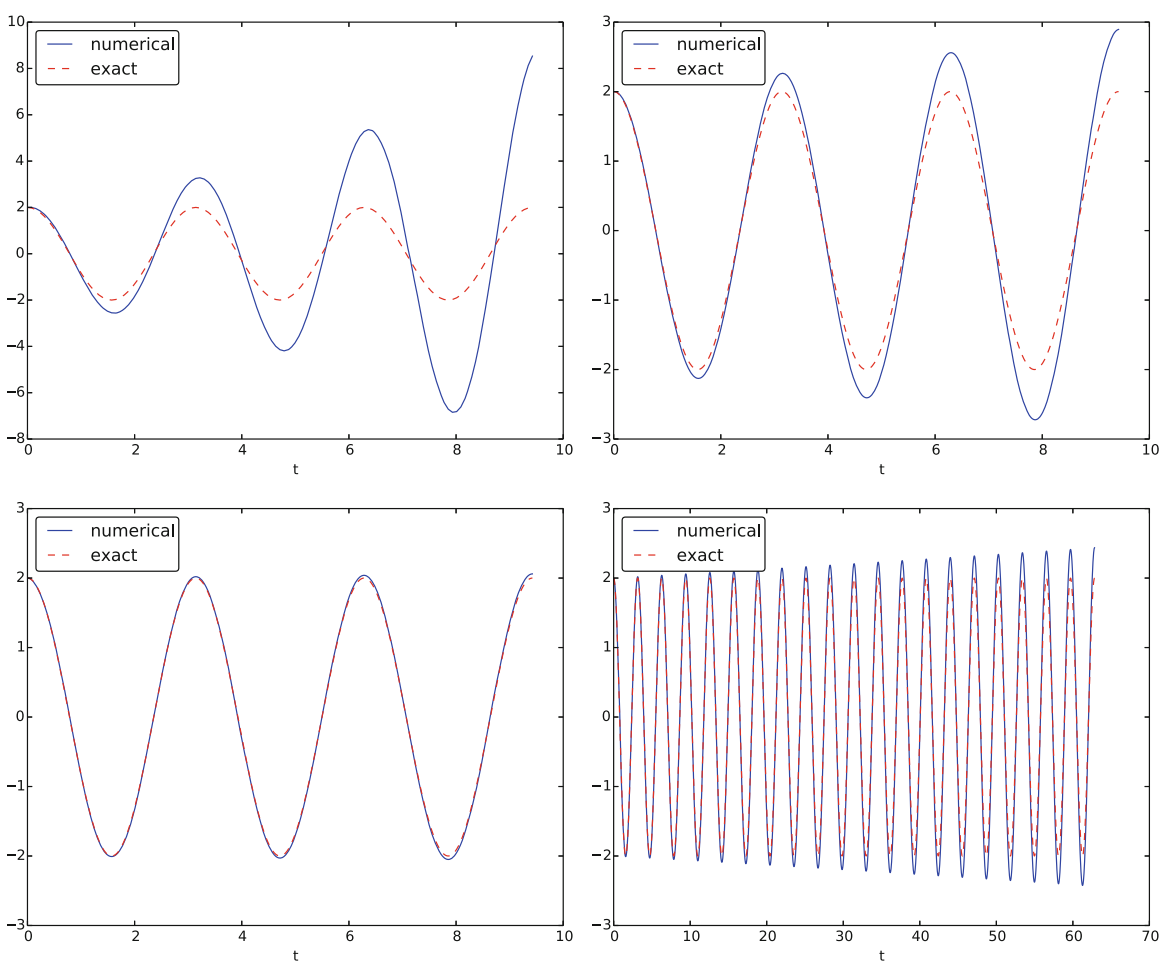

Fig. 4.17 Simulation of an oscillating system with different time steps. Upper left: 40 steps per oscillation period. Upper right: 160 steps per period. Lower left: 2000 steps per period. Lower right: 2000 steps per period, but longer simulation

calculations show that the program is seemingly correct. (Later, we can use such values to construct a unit test and a corresponding test function.)

The next step is to reduce the discretization parameter $\Delta t$ and see if the results become more accurate. Figure 4.17 shows the numerical and exact solution for the cases $\Delta t=P / 40, P / 160, P / 2000$. The results clearly become better, and the finest resolution gives graphs that cannot be visually distinguished. Nevertheless, the finest resolution involves 6000 computational intervals in total, which is considered quite much. This is no problem on a modern laptop, however, as the computations take just a fraction of a second.

Although 2000 intervals per oscillation period seem sufficient for an accurate numerical solution, the lower right graph in Fig. 4.17 shows that if we increase the simulation time, here to 20 periods, there is a little growth of the amplitude, which becomes significant over time. The conclusion is that the Forward Euler method has a fundamental problem with its growing amplitudes, and that a very small $\Delta t$ is required to achieve satisfactory results. The longer the simulation is, the smaller $\Delta t$ has to be. It is certainly time to look for more effective numerical methods! 

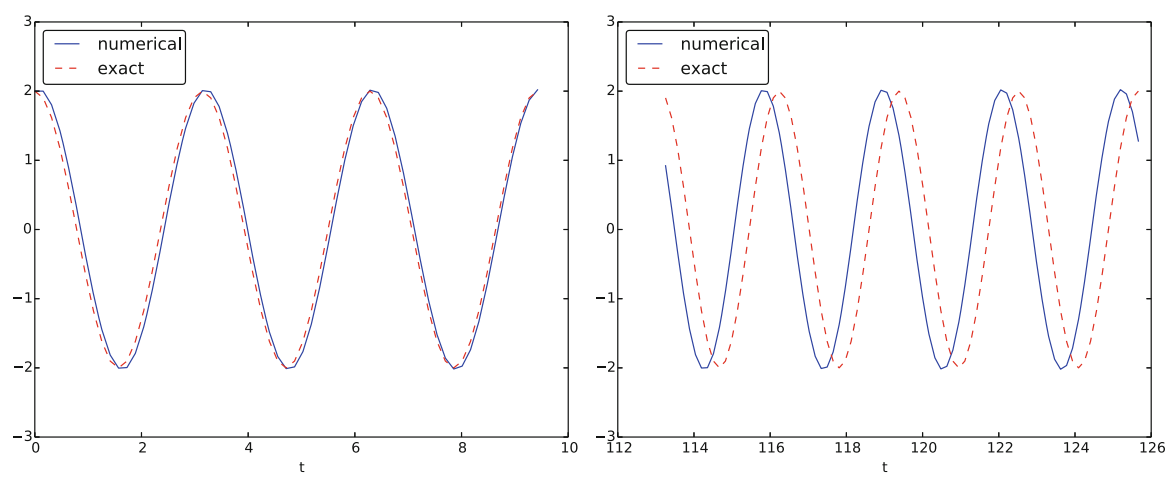

Fig. 4.18 Adjusted method: first three periods (left) and period 36-40 (right)

\subsubsection{A Magic Fix of the Numerical Method}

In the Forward Euler scheme,

$$
\begin{aligned}
& u^{n+1}=u^{n}+\Delta t v^{n}, \\
& v^{n+1}=v^{n}-\Delta t \omega^{2} u^{n},
\end{aligned}
$$

we can replace $u^{n}$ in the last equation by the recently computed value $u^{n+1}$ from the first equation:

$$
\begin{aligned}
& u^{n+1}=u^{n}+\Delta t v^{n} \\
& v^{n+1}=v^{n}-\Delta t \omega^{2} u^{n+1}
\end{aligned}
$$

Before justifying this fix more mathematically, let us try it on the previous example. The results appear in Fig. 4.18. We see that the amplitude does not grow, but the phase is not entirely correct. After 40 periods (Fig. 4.18 right) we see a significant difference between the numerical and the exact solution. Decreasing $\Delta t$ decreases the error. For example, with 2000 intervals per period, we only see a small phase error even after 50,000 periods (!). We can safely conclude that the fix results in an excellent numerical method!

Let us interpret the adjusted scheme mathematically. First we order (4.49)-(4.50) such that the difference approximations to derivatives become transparent:

$$
\begin{aligned}
& \frac{u^{n+1}-u^{n}}{\Delta t}=v^{n}, \\
& \frac{v^{n+1}-v^{n}}{\Delta t}=-\omega^{2} u^{n+1} .
\end{aligned}
$$

We interpret (4.51) as the differential equation sampled at mesh point $t_{n}$, because we have $v^{n}$ on the right-hand side. The left-hand side is then a forward difference or Forward Euler approximation to the derivative $u^{\prime}$, see Fig. 4.2. On the other hand, 


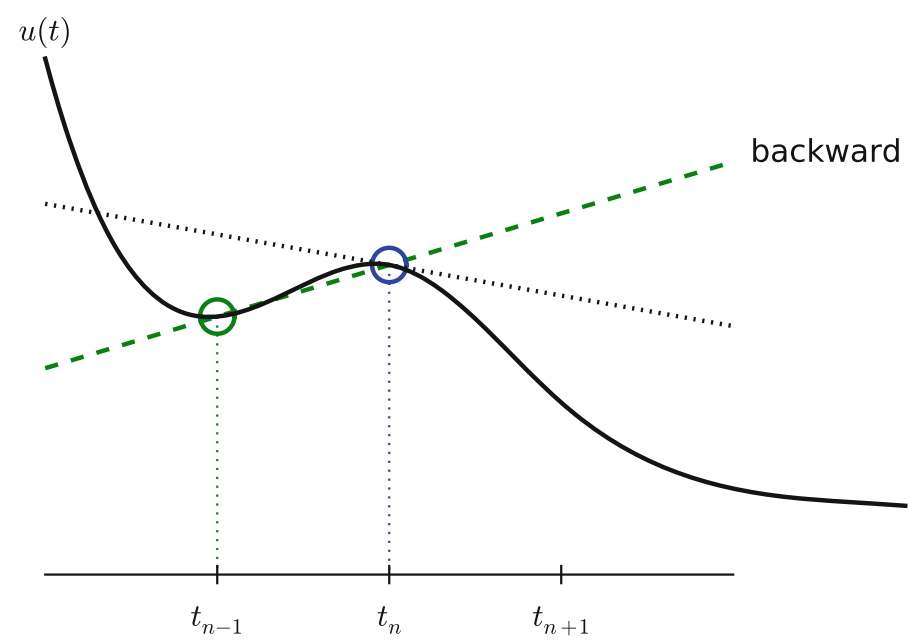

Fig. 4.19 Illustration of a backward difference approximation to the derivative

we interpret (4.52) as the differential equation sampled at mesh point $t_{n+1}$, since we have $u^{n+1}$ on the right-hand side. In this case, the difference approximation on the left-hand side is a backward difference,

$$
v^{\prime}\left(t_{n+1}\right) \approx \frac{v^{n+1}-v^{n}}{\Delta t} \quad \text { or } \quad v^{\prime}\left(t_{n}\right) \approx \frac{v^{n}-v^{n-1}}{\Delta t} .
$$

Figure 4.19 illustrates the backward difference. The error in the backward difference is proportional to $\Delta t$, the same as for the forward difference (but the proportionality constant in the error term has different sign). The resulting discretization method for (4.52) is often referred to as a Backward Euler scheme.

To summarize, using a forward difference for the first equation and a backward difference for the second equation results in a much better method than just using forward differences in both equations.

The standard way of expressing this scheme in physics is to change the order of the equations,

$$
\begin{aligned}
& v^{\prime}=-\omega^{2} u, \\
& u^{\prime}=v,
\end{aligned}
$$

and apply a forward difference to (4.53) and a backward difference to (4.54):

$$
\begin{aligned}
& v^{n+1}=v^{n}-\Delta t \omega^{2} u^{n}, \\
& u^{n+1}=u^{n}+\Delta t v^{n+1} .
\end{aligned}
$$

That is, first the velocity $v$ is updated and then the position $u$, using the most recently computed velocity. There is no difference between (4.55)-(4.56) and (4.49)(4.50) with respect to accuracy, so the order of the original differential equations 
does not matter. The scheme (4.55)-(4.56) goes under the names Semi-implicit Euler $^{4}$ or Euler-Cromer. The implementation of (4.55)-(4.56) is found in the file osc_EC.m. The core of the code goes like

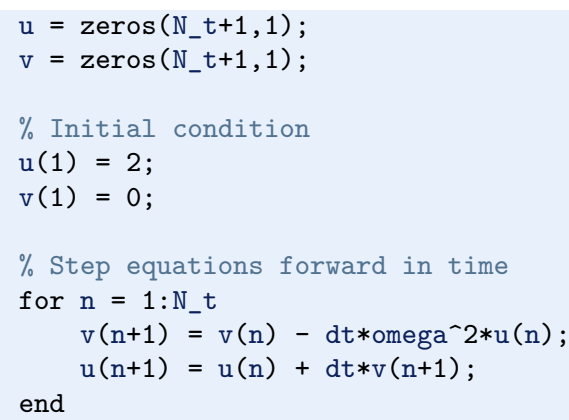

\subsubsection{The 2nd-Order Runge-Kutta Method (or Heun's Method)}

A very popular method for solving scalar and vector ODEs of first order is the 2nd-order Runge-Kutta method (RK2), also known as Heun's method. The idea, first thinking of a scalar ODE, is to form a centered difference approximation to the derivative between two time points:

$$
u^{\prime}\left(t_{n}+\frac{1}{2} \Delta t\right) \approx \frac{u^{n+1}-u^{n}}{\Delta t} .
$$

The centered difference formula is visualized in Fig. 4.20. The error in the centered difference is proportional to $\Delta t^{2}$, one order higher than the forward and backward differences, which means that if we halve $\Delta t$, the error is more effectively reduced in the centered difference since it is reduced by a factor of four rather than two.

The problem with such a centered scheme for the general ODE $u^{\prime}=f(u, t)$ is that we get

$$
\frac{u^{n+1}-u^{n}}{\Delta t}=f\left(u^{n+\frac{1}{2}}, t_{n+\frac{1}{2}}\right)
$$

which leads to difficulties since we do not know what $u^{n+\frac{1}{2}}$ is. However, we can approximate the value of $f$ between two time levels by the arithmetic average of the values at $t_{n}$ and $t_{n+1}$ :

$$
f\left(u^{n+\frac{1}{2}}, t_{n+\frac{1}{2}}\right) \approx \frac{1}{2}\left(f\left(u^{n}, t_{n}\right)+f\left(u^{n+1}, t_{n+1}\right)\right) .
$$

This results in

$$
\frac{u^{n+1}-u^{n}}{\Delta t}=\frac{1}{2}\left(f\left(u^{n}, t_{n}\right)+f\left(u^{n+1}, t_{n+1}\right)\right),
$$

\footnotetext{
${ }^{4}$ http://en.wikipedia.org/wiki/Semi-implicit_Euler_method
} 


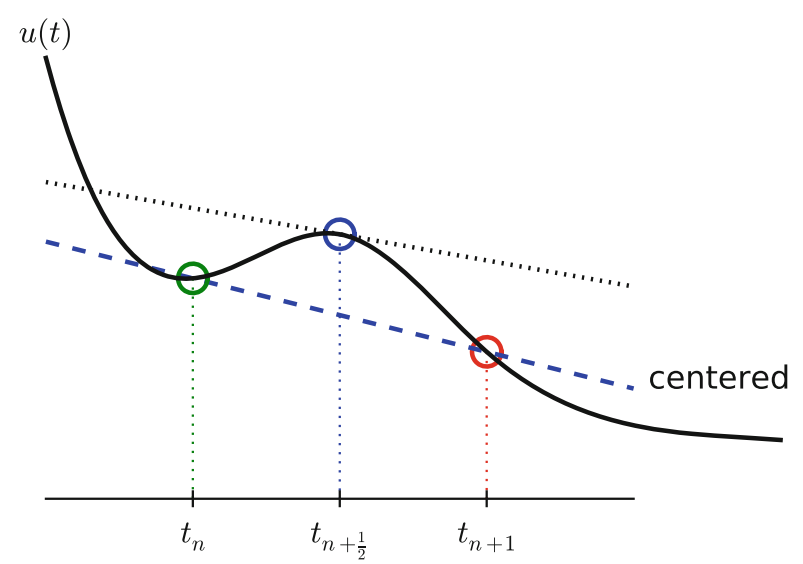

Fig. 4.20 Illustration of a centered difference approximation to the derivative

which in general is a nonlinear algebraic equation for $u^{n+1}$ if $f(u, t)$ is not a linear function of $u$. To deal with the unknown term $f\left(u^{n+1}, t_{n+1}\right)$, without solving nonlinear equations, we can approximate or predict $u^{n+1}$ using a Forward Euler step:

$$
u^{n+1}=u^{n}+\Delta t f\left(u^{n}, t_{n}\right) .
$$

This reasoning gives rise to the method

$$
\begin{aligned}
u^{*} & =u^{n}+\Delta t f\left(u^{n}, t_{n}\right), \\
u^{n+1} & =u^{n}+\frac{\Delta t}{2}\left(f\left(u^{n}, t_{n}\right)+f\left(u^{*}, t_{n+1}\right)\right) .
\end{aligned}
$$

The scheme applies to both scalar and vector ODEs.

For an oscillating system with $f=\left(v,-\omega^{2} u\right)$ the file osc_Heun.mimplements this method. The demo script demo_osc_Heun.m runs the simulation for 10 periods with 20 time steps per period. The corresponding numerical and exact solutions are shown in Fig. 4.21. We see that the amplitude grows, but not as much as for the Forward Euler method. However, the Euler-Cromer method is much better!

We should add that in problems where the Forward Euler method gives satisfactory approximations, such as growth/decay problems or the SIR model, the 2nd-order Runge-Kutta method or Heun's method, usually works considerably better and produces greater accuracy for the same computational cost. It is therefore a very valuable method to be aware of, although it cannot compete with the EulerCromer scheme for oscillation problems. The derivation of the RK2/Heun scheme is also good general training in "numerical thinking".

\subsubsection{Software for Solving ODEs}

Matlab and Octave users have a handful of functions for solving ODEs, e.g. the popular methods ode 45 and ode23s. To illustrate, we may use ode 45 to solve the simple problem $u^{\prime}=u, u(0)=2$, for 100 time steps until $t=4$ : 


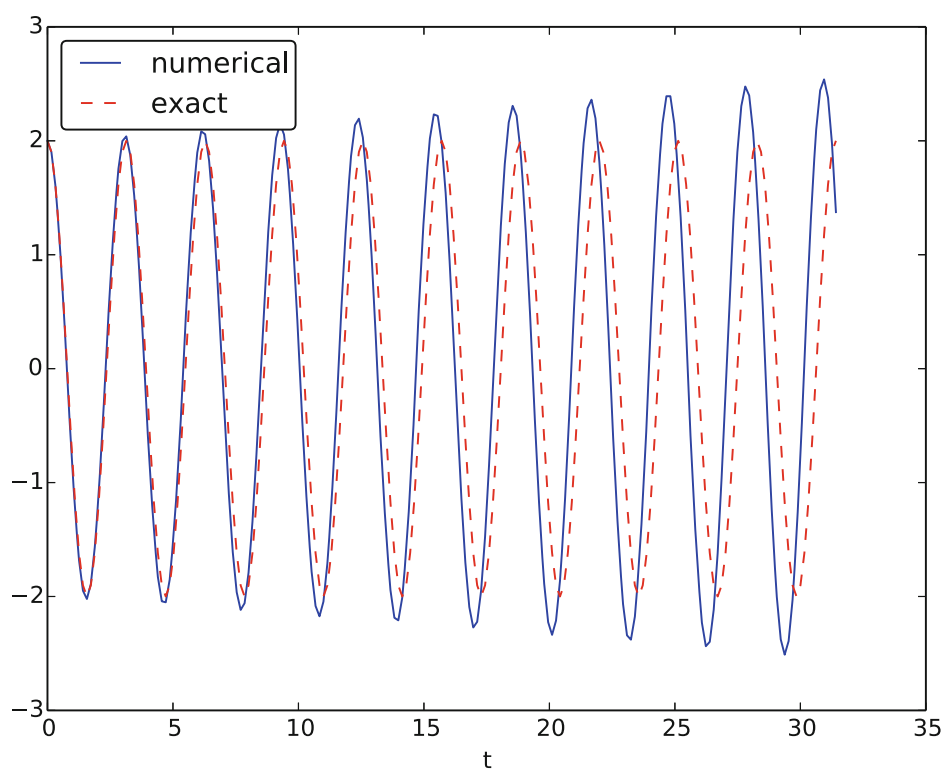

Fig. 4.21 Simulation of 10 periods of oscillations by Heun's method

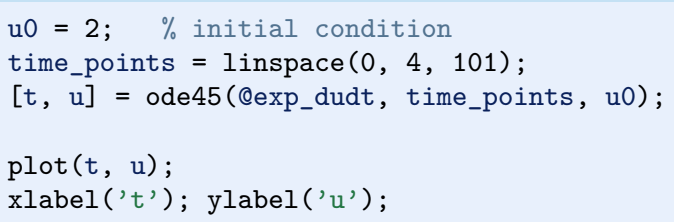

Here, ode 45 is called with three parameters. The first one, @exp_dudt, is a handle to a function that specifies the right hand side of the ODE, i.e., $f(u, t)$. In the present example, it reads

function dudt $=\exp \_\operatorname{dudt}(t, u)$

dudt $=u$

The second parameter, time_points, is an array that gives the time points on the interval where we want the solution to be reported. Alternatively, this second parameter could have been given as [0 4], which just specifies the interval, giving no directions to Matlab as to where (on the interval) the solution should be found. The third parameter, u0, just states the initial condition.

Other ODE solvers in Matlab work in a similar fashion. Several ODEs may also be solved with one function call and parameters may be included.

There is a jungle of methods for solving ODEs, and it would be nice to have easy access to implementations of a wide range of methods, especially the sophisticated and complicated adaptive methods (like ode 45 and ode23s above) that adjusts $\Delta t$ 
automatically to obtain a prescribed accuracy. The Python package Odespy ${ }^{5}$ gives easy access to a lot of numerical methods for ODEs.

The simplest possible example on using Odespy is to solve the same problem that we just looked at, i.e., $u^{\prime}=u, u(0)=2$, for 100 time steps until $t=4$ :

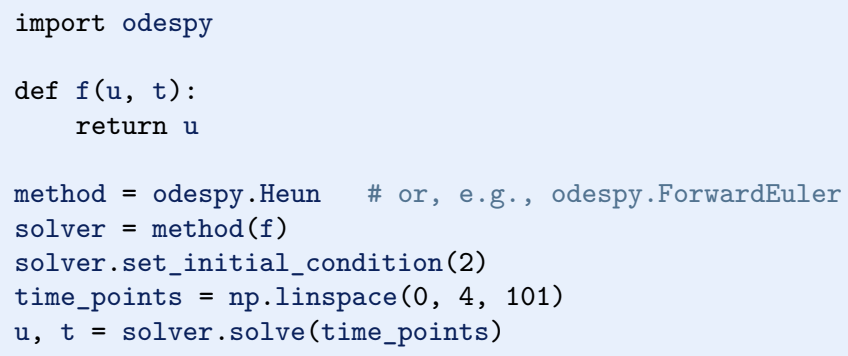

In other words, you define your right-hand side function $f(u, t)$, initialize an Odespy solver object, set the initial condition, compute a collection of time points where you want the solution, and ask for the solution. The returned arrays $u$ and $t$ can be plotted directly: plot ( $t, u)$.

\section{Warning}

Note that Odespy must be operated from Python, so you need to learn some basic Python to make use of this software. The type of Python programming you need to learn has a syntax very close to that of Matlab.

A nice feature of Odespy is that problem parameters can be arguments to the user's $\mathrm{f}\left(\mathrm{u}, \mathrm{t}\right.$ ) function. For example, if our ODE problem is $u^{\prime}=-a u+b$, with two problem parameters $a$ and $b$, we may write our $\mathrm{f}$ function as

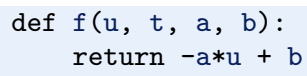

The extra, problem-dependent arguments $\mathrm{a}$ and $\mathrm{b}$ can be transferred to this function if we collect their values in a list or tuple when creating the Odespy solver and use the $f_{\text {_args }}$ argument:

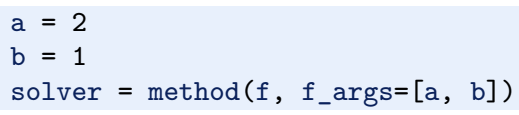

This is a good feature because problem parameters must otherwise be global variables - now they can be arguments in our right-hand side function in a natural way. Exercise 4.16 asks you to make a complete implementation of this problem and plot the solution.

Using Odespy to solve oscillation ODEs like $u^{\prime \prime}+\omega^{2} u=0$, reformulated as a system $u^{\prime}=v$ and $v^{\prime}=-\omega^{2} u$, is done as follows. We specify a given number

${ }^{5}$ https://github.com/hplgit/odespy 
of time steps per period and compute the associated time steps and end time of the simulation $(\mathrm{T})$, given a number of periods to simulate:

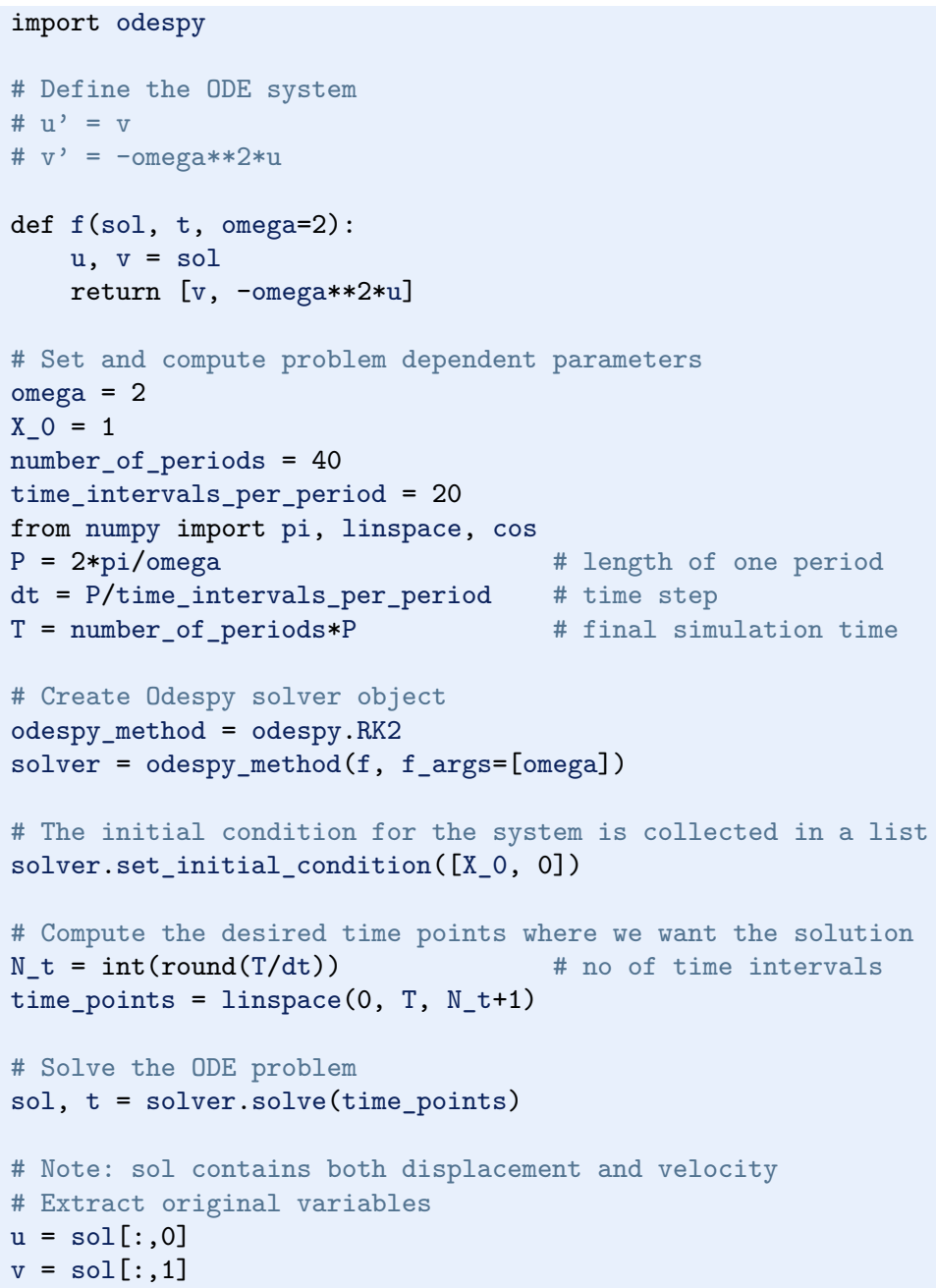

The last two statements are important since our two functions $u$ and $v$ in the ODE system are packed together in one array inside the Odespy solver. The solution of the ODE system is returned as a two-dimensional array where the first column ( $\operatorname{sol}[:, 0])$ stores $u$ and the second $(\operatorname{sol}[:, 1])$ stores $v$. Plotting $u$ and $v$ is a matter of running plot ( $t, u, t, v)$.

\section{Remark}

In the right-hand side function we write $f$ (sol, $t$, omega) instead of $f(u$, $t$, omega) to indicate that the solution sent to $f$ is a solution at time $t$ where the values of $u$ and $v$ are packed together: sol $=[u, v]$. We might well use $u$ as argument: 


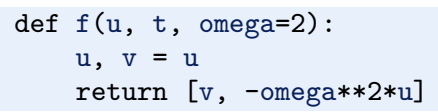

This just means that we redefine the name $u$ inside the function to mean the solution at time $t$ for the first component of the ODE system.

To switch to another numerical method, just substitute RK2 by the proper name of the desired method. Typing pydoc odespy in the terminal window brings up a list of all the implemented methods. This very simple way of choosing a method suggests an obvious extension of the code above: we can define a list of methods, run all methods, and compare their $u$ curves in a plot. As Odespy also contains the Euler-Cromer scheme, we rewrite the system with $v^{\prime}=-\omega^{2} u$ as the first ODE and $u^{\prime}=v$ as the second ODE, because this is the standard choice when using the Euler-Cromer method (also in Odespy):

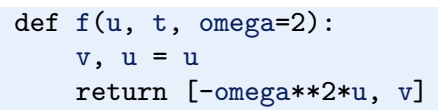

This change of equations also affects the initial condition: the first component is zero and second is $\mathrm{X}_{-} 0$ so we need to pass the list [0, $\mathrm{X}_{-} 0$ ] to solver.set_ initial_condition.

The code osc_odespy · py contains the details:

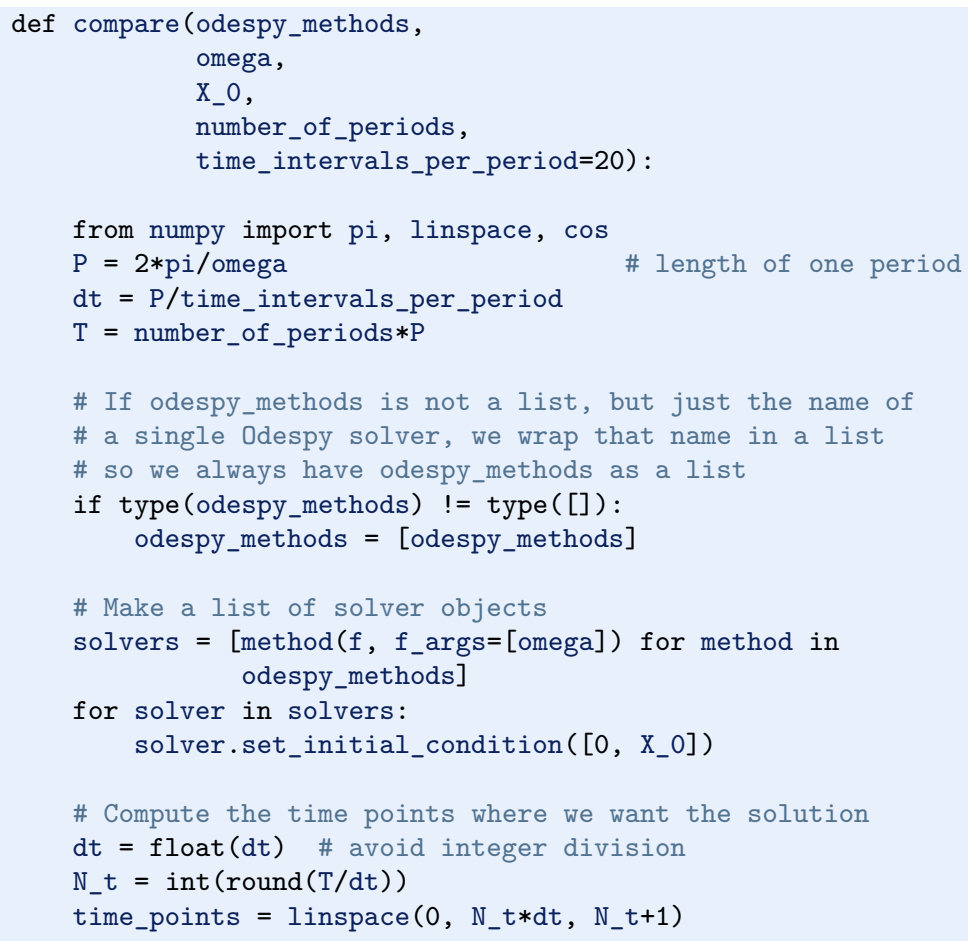




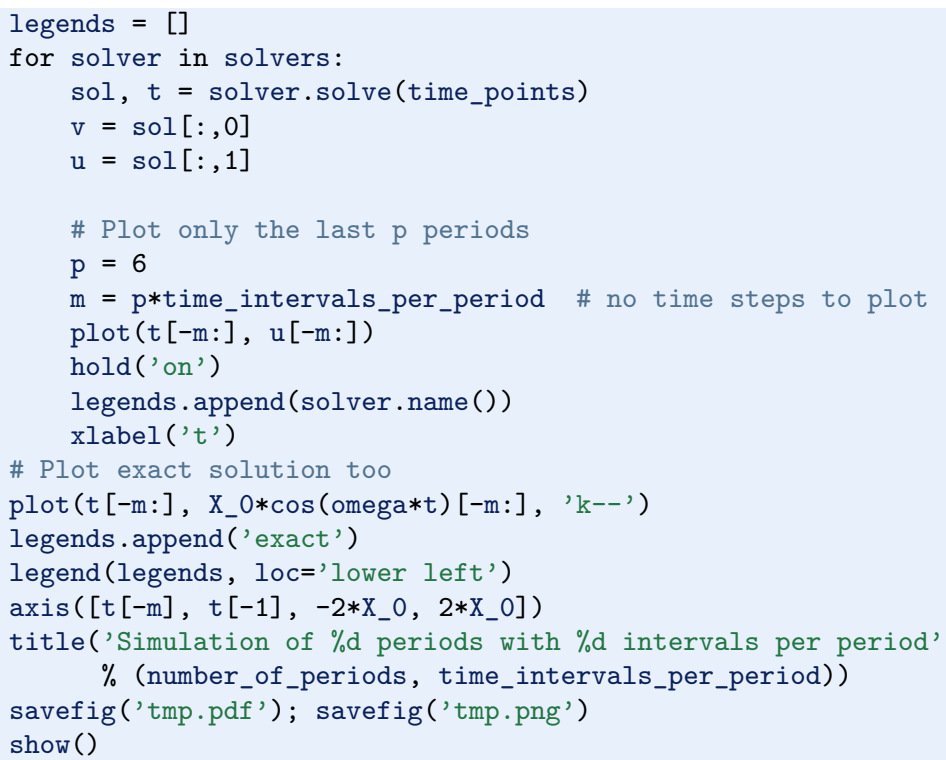

A new feature in this code is the ability to plot only the last p periods, which allows us to perform long time simulations and watch the end results without a cluttered plot with too many periods. The syntax $t[-\mathrm{m}:]$ plots the last $\mathrm{m}$ elements in $\mathrm{t}$ (a negative index in Python arrays/lists counts from the end).

We may compare Heun's method (or equivalently the RK2 method) with the Euler-Cromer scheme:

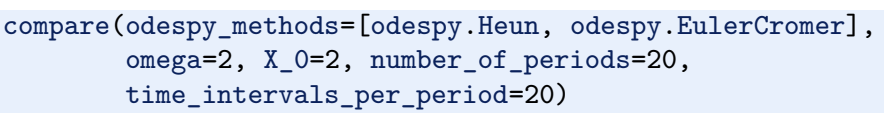

Figure 4.22 shows how Heun's method (the blue line with small disks) has considerable error in both amplitude and phase already after 14-20 periods (upper left), but using three times as many time steps makes the curves almost equal (upper right). However, after 194-200 periods the errors have grown (lower left), but can be sufficiently reduced by halving the time step (lower right).

With all the methods in Odespy at hand, it is now easy to start exploring other methods, such as backward differences instead of the forward differences used in the Forward Euler scheme. Exercise 4.17 addresses that problem.

Odespy contains quite sophisticated adaptive methods where the user is "guaranteed" to get a solution with prescribed accuracy. There is no mathematical guarantee, but the error will for most cases not deviate significantly from the user's tolerance that reflects the accuracy. A very popular method of this type is the Runge-Kutta-Fehlberg method, which runs a 4th-order Runge-Kutta method and uses a 5th-order Runge-Kutta method to estimate the error so that $\Delta t$ can be adjusted to keep the error below a tolerance. This method is also widely known as ode 45, because that is the name of the function implementing the method in Matlab. We can easily test the Runge-Kutta-Fehlberg method as soon as we know the corresponding Odespy name, which is RKFehlberg: 

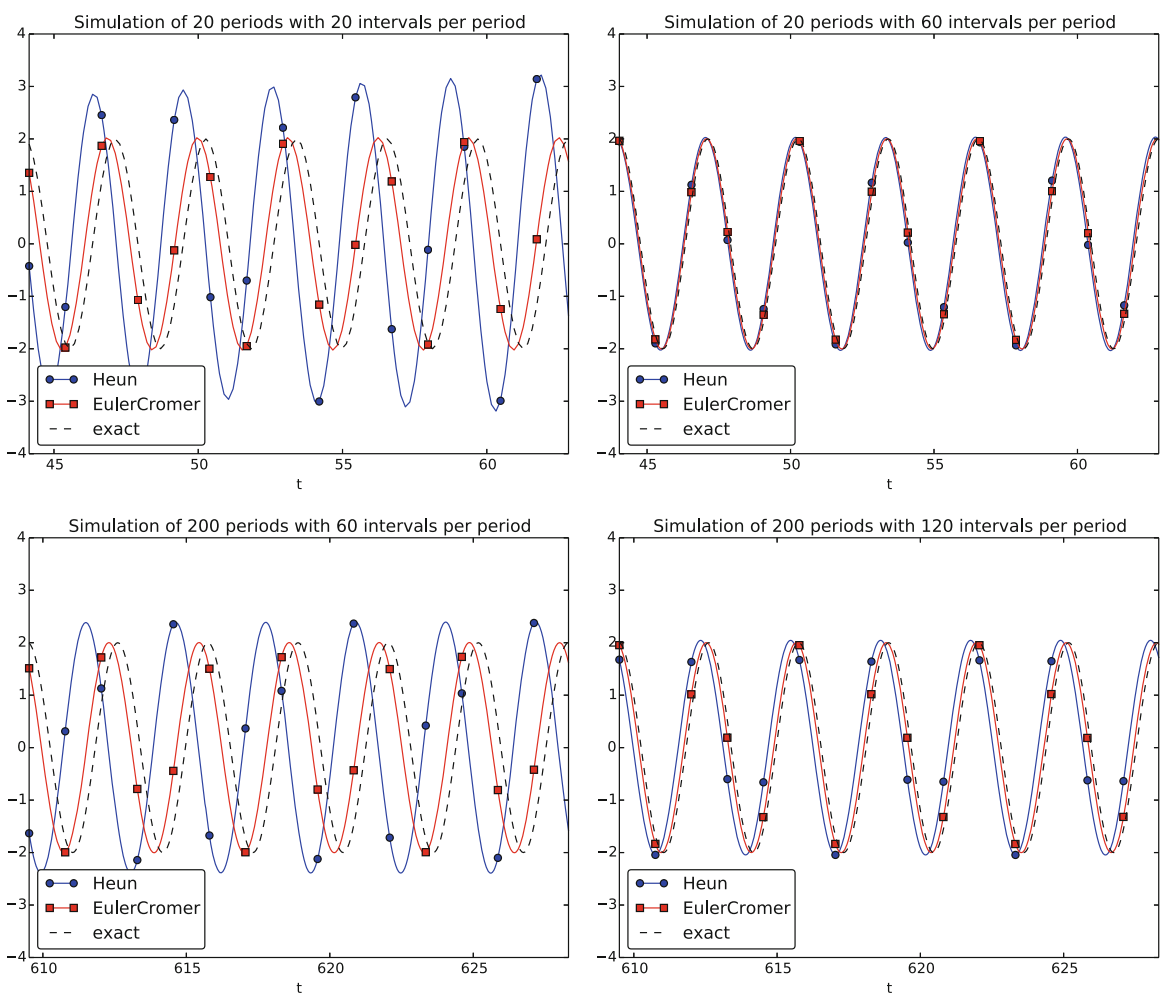

Fig. 4.22 Illustration of the impact of resolution (time steps per period) and length of simulation

compare (odespy_methods=[odespy.EulerCromer, odespy.RKFehlberg] , omega $=2, X_{-} 0=2$, number_of_periods $=200$, time_intervals_per_period=40)

Note that the time_intervals_per_period argument refers to the time points where we want the solution. These points are also the ones used for numerical computations in the odespy. EulerCromer solver, while the odespy. RKFehlberg solver will use an unknown set of time points since the time intervals are adjusted as the method runs. One can easily look at the points actually used by the method as these are available as an array solver.t_all (but plotting or examining the points requires modifications inside the compare method).

Figure 4.23 shows a computational example where the Runge-Kutta-Fehlberg method is clearly superior to the Euler-Cromer scheme in long time simulations, but the comparison is not really fair because the Runge-Kutta-Fehlberg method applies about twice as many time steps in this computation and performs much more work per time step. It is quite a complicated task to compare two so different methods in a fair way so that the computational work versus accuracy is scientifically well reported. 


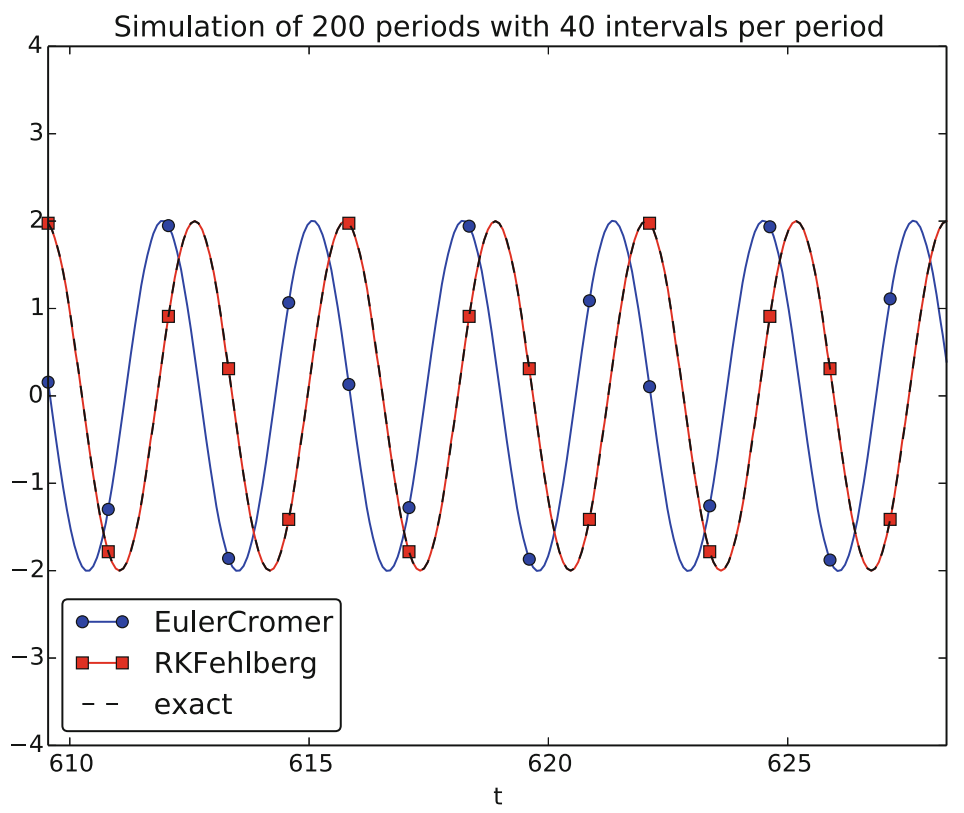

Fig. 4.23 Comparison of the Runge-Kutta-Fehlberg adaptive method against the Euler-Cromer scheme for a long time simulation (200 periods)

\subsubsection{The 4th-Order Runge-Kutta Method}

The 4th-order Runge-Kutta method (RK4) is clearly the most widely used method to solve ODEs. Its power comes from high accuracy even with not so small time steps.

The algorithm We first just state the four-stage algorithm:

$$
u^{n+1}=u^{n}+\frac{\Delta t}{6}\left(f^{n}+2 \hat{f}^{n+\frac{1}{2}}+2 \tilde{f}^{n+\frac{1}{2}}+\bar{f}^{n+1}\right)
$$

where

$$
\begin{aligned}
& \hat{f}^{n+\frac{1}{2}}=f\left(u^{n}+\frac{1}{2} \Delta t f^{n}, t_{n+\frac{1}{2}}\right), \\
& \tilde{f}^{n+\frac{1}{2}}=f\left(u^{n}+\frac{1}{2} \Delta t \hat{f}^{n+\frac{1}{2}}, t_{n+\frac{1}{2}}\right), \\
& \bar{f}^{n+1}=f\left(u^{n}+\Delta t \tilde{f}^{n+\frac{1}{2}}, t_{n+1}\right) .
\end{aligned}
$$

Application We can run the same simulation as in Figs. 4.16, 4.18, and 4.21, for 40 periods. The 10 last periods are shown in Fig. 4.24. The results look as impressive as those of the Euler-Cromer method. 


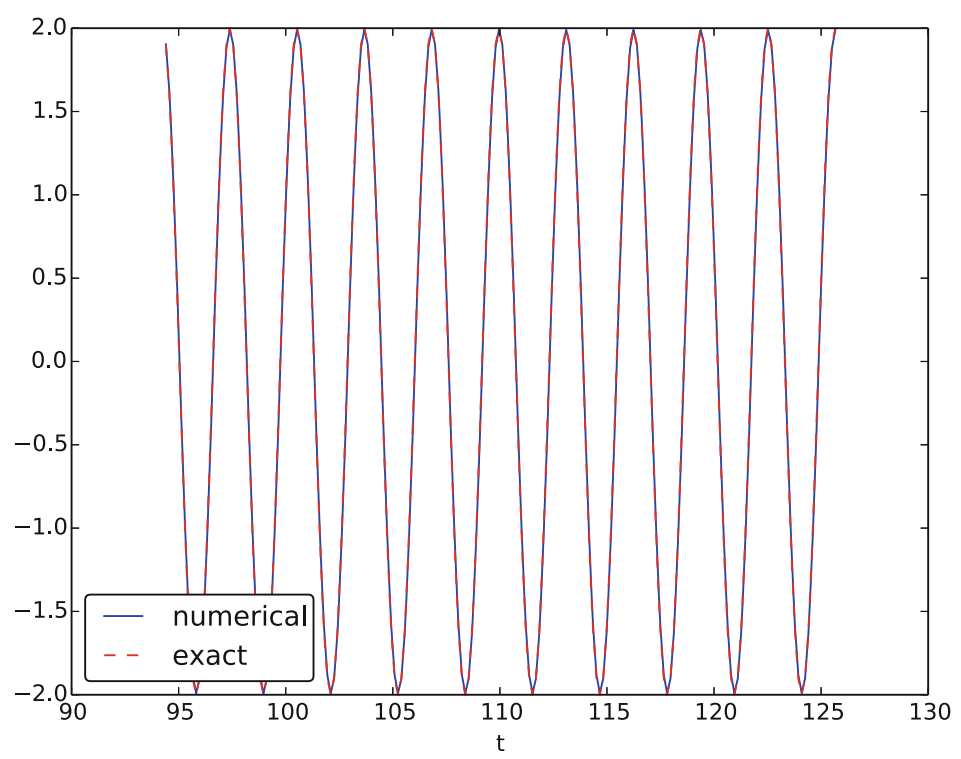

Fig. 4.24 The last 10 of 40 periods of oscillations by the 4th-order Runge-Kutta method

Implementation The stages in the 4th-order Runge-Kutta method can easily be implemented as a modification of the osc_Heun.py code. Alternatively, one can use the osc_odespy.py code by just providing the argument odespy_methods= [odespy.RK4] to the compare function.

Derivation The derivation of the 4th-order Runge-Kutta method can be presented in a pedagogical way that brings many fundamental elements of numerical discretization techniques together and that illustrates many aspects of "numerical thinking" when constructing approximate solution methods.

We start with integrating the general ODE $u^{\prime}=f(u, t)$ over a time step, from $t_{n}$ to $t_{n+1}$,

$$
u\left(t_{n+1}\right)-u\left(t_{n}\right)=\int_{t_{n}}^{t_{n+1}} f(u(t), t) d t .
$$

The goal of the computation is $u\left(t_{n+1}\right)\left(u^{n+1}\right)$, while $u\left(t_{n}\right)\left(u^{n}\right)$ is the most recently known value of $u$. The challenge with the integral is that the integrand involves the unknown $u$ between $t_{n}$ and $t_{n+1}$.

The integral can be approximated by the famous Simpson's rule ${ }^{6}$ :

$$
\int_{t_{n}}^{t_{n+1}} f(u(t), t) d t \approx \frac{\Delta t}{6}\left(f^{n}+4 f^{n+\frac{1}{2}}+f^{n+1}\right) .
$$

\footnotetext{
${ }^{6}$ http://en.wikipedia.org/wiki/Simpson's_rule
} 
The problem with this formula is that we do not know $f^{n+\frac{1}{2}}=f\left(u^{n+\frac{1}{2}}, t_{n+\frac{1}{2}}\right)$ and $f^{n+1}=\left(u^{n+1}, t_{n+1}\right)$ as only $u^{n}$ is available and only $f^{n}$ can then readily be computed.

To proceed, the idea is to use various approximations for $f^{n+\frac{1}{2}}$ and $f^{n+1}$ based on using well-known schemes for the ODE in the intervals $\left[t_{n}, t_{n+\frac{1}{2}}\right]$ and $\left[t_{n}, t_{n+1}\right]$. Let us split the integral into four terms:

$$
\int_{t_{n}}^{t_{n+1}} f(u(t), t) d t \approx \frac{\Delta t}{6}\left(f^{n}+2 \hat{f}^{n+\frac{1}{2}}+2 \tilde{f}^{n+\frac{1}{2}}+\bar{f}^{n+1}\right)
$$

where $\hat{f}^{n+\frac{1}{2}}, \tilde{f}^{n+\frac{1}{2}}$, and $\bar{f}^{n+1}$ are approximations to $f^{n+\frac{1}{2}}$ and $f^{n+1}$ that can utilize already computed quantities. For $\hat{f}^{n+\frac{1}{2}}$ we can simply apply an approximation to $u^{n+\frac{1}{2}}$ based on a Forward Euler step of size $\frac{1}{2} \Delta t$ :

$$
\hat{f}^{n+\frac{1}{2}}=f\left(u^{n}+\frac{1}{2} \Delta t f^{n}, t_{n+\frac{1}{2}}\right)
$$

This formula provides a prediction of $f^{n+\frac{1}{2}}$, so we can for $\tilde{f}^{n+\frac{1}{2}}$ try a Backward Euler method to approximate $u^{n+\frac{1}{2}}$ :

$$
\tilde{f}^{n+\frac{1}{2}}=f\left(u^{n}+\frac{1}{2} \Delta t \hat{f}^{n+\frac{1}{2}}, t_{n+\frac{1}{2}}\right) .
$$

With $\tilde{f}^{n+\frac{1}{2}}$ as an approximation to $f^{n+\frac{1}{2}}$, we can for the final term $\bar{f}^{n+1}$ use a midpoint method (or central difference, also called a Crank-Nicolson method) to approximate $u^{n+1}$ :

$$
\bar{f}^{n+1}=f\left(u^{n}+\Delta t \hat{f}^{n+\frac{1}{2}}, t_{n+1}\right) .
$$

We have now used the Forward and Backward Euler methods as well as the centered difference approximation in the context of Simpson's rule. The hope is that the combination of these methods yields an overall time-stepping scheme from $t_{n}$ to $t_{n}+1$ that is much more accurate than the individual steps which have errors proportional to $\Delta t$ and $\Delta t^{2}$. This is indeed true: the numerical error goes in fact like $C \Delta t^{4}$ for a constant $C$, which means that the error approaches zero very quickly as we reduce the time step size, compared to the Forward Euler method (error $\sim \Delta t$ ), the Euler-Cromer method (error $\sim \Delta t$ ) or the 2nd-order Runge-Kutta, or Heun's, method (error $\left.\sim \Delta t^{2}\right)$.

Note that the 4th-order Runge-Kutta method is fully explicit so there is never any need to solve linear or nonlinear algebraic equations, regardless of what $f$ looks like. However, the stability is conditional and depends on $f$. There is a large family of implicit Runge-Kutta methods that are unconditionally stable, but require solution of algebraic equations involving $f$ at each time step. The Odespy package has support for a lot of sophisticated explicit Runge-Kutta methods, but not yet implicit Runge-Kutta methods. 


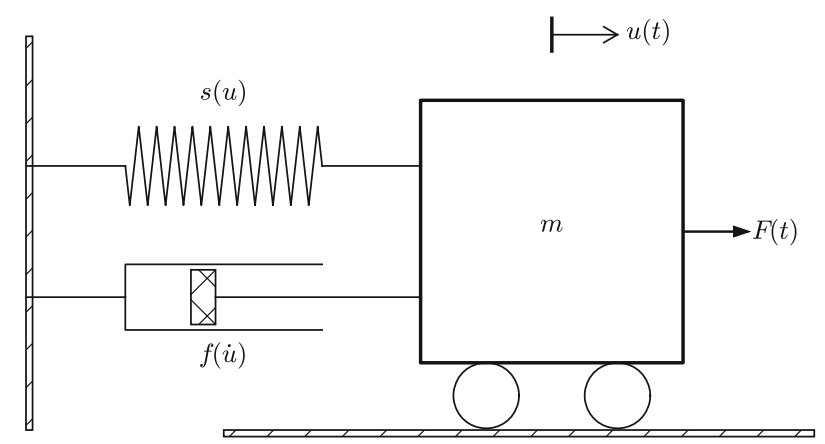

Fig.4.25 General oscillating system

\subsubsection{More Effects: Damping, Nonlinearity, and External Forces}

Our model problem $u^{\prime \prime}+\omega^{2} u=0$ is the simplest possible mathematical model for oscillating systems. Nevertheless, this model makes strong demands to numerical methods, as we have seen, and is very useful as a benchmark for evaluating the performance of numerical methods.

Real-life applications involve more physical effects, which lead to a differential equation with more terms and also more complicated terms. Typically, one has a damping force $f\left(u^{\prime}\right)$ and a spring force $s(u)$. Both these forces may depend nonlinearly on their argument, $u^{\prime}$ or $u$. In addition, environmental forces $F(t)$ may act on the system. For example, the classical pendulum has a nonlinear "spring" or restoring force $s(u) \sim \sin (u)$, and air resistance on the pendulum leads to a damping force $f\left(u^{\prime}\right) \sim\left|u^{\prime}\right| u^{\prime}$. Examples on environmental forces include shaking of the ground (e.g., due to an earthquake) as well as forces from waves and wind.

With three types of forces on the system: $F, f$, and $s$, the sum of forces is written $F(t)-f\left(u^{\prime}\right)-s(u)$. Note the minus sign in front of $f$ and $s$, which indicates that these functions are defined such that they represent forces acting against the motion. For example, springs attached to the wheels in a car are combined with effective dampers, each providing a damping force $f\left(u^{\prime}\right)=b u^{\prime}$ that acts against the spring velocity $u^{\prime}$. The corresponding physical force is then $-f:-b u^{\prime}$, which points downwards when the spring is being stretched (and $u^{\prime}$ points upwards), while $-f$ acts upwards when the spring is being compressed (and $u^{\prime}$ points downwards).

Figure 4.25 shows an example of a mass $m$ attached to a potentially nonlinear spring and dashpot, and subject to an environmental force $F(t)$. Nevertheless, our general model can equally well be a pendulum as in Fig. 4.26 with $s(u)=m g \sin \theta$ and $f(\dot{u})=\frac{1}{2} C_{D} A \varrho \dot{\theta}|\dot{\theta}|$ (where $C_{D}=0.4, A$ is the cross sectional area of the body, and $\varrho$ is the density of air).

Newton's second law for the system can be written with the mass times acceleration on the left-hand side and the forces on the right-hand side:

$$
m u^{\prime \prime}=F(t)-f\left(u^{\prime}\right)-s(u) .
$$

This equation is, however, more commonly reordered to

$$
m u^{\prime \prime}+f\left(u^{\prime}\right)+s(u)=F(t) .
$$




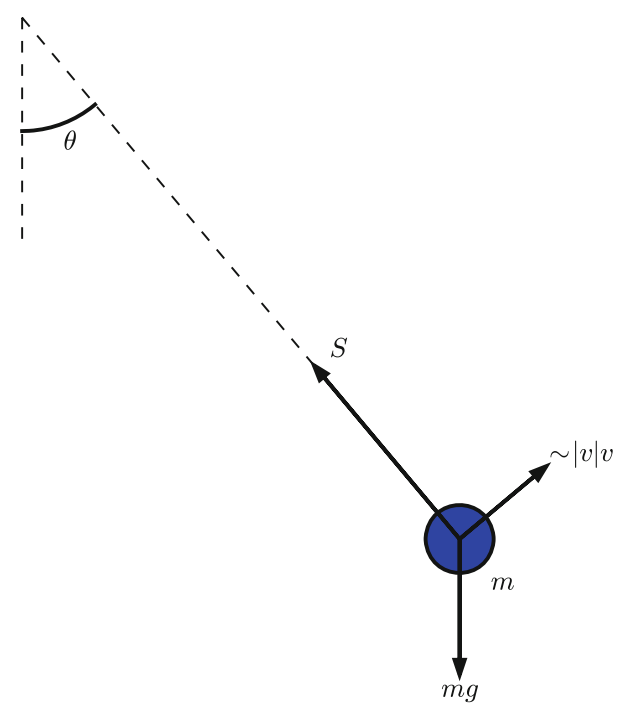

Fig.4.26 A pendulum with forces

Because the differential equation is of second order, due to the term $u^{\prime \prime}$, we need two initial conditions:

$$
u(0)=U_{0}, \quad u^{\prime}(0)=V_{0} .
$$

Note that with the choices $f\left(u^{\prime}\right)=0, s(u)=k u$, and $F(t)=0$ we recover the original ODE $u^{\prime \prime}+\omega^{2} u=0$ with $\omega=\sqrt{k / m}$.

How can we solve (4.66)? As for the simple ODE $u^{\prime \prime}+\omega^{2} u=0$, we start by rewriting the second-order ODE as a system of two first-order ODEs:

$$
\begin{aligned}
v^{\prime} & =\frac{1}{m}(F(t)-s(u)-f(v)), \\
u^{\prime} & =v
\end{aligned}
$$

The initial conditions become $u(0)=U_{0}$ and $v(0)=V_{0}$.

Any method for a system of first-order ODEs can be used to solve for $u(t)$ and $v(t)$.

The Euler-Cromer scheme An attractive choice from an implementational, accuracy, and efficiency point of view is the Euler-Cromer scheme where we take a forward difference in (4.68) and a backward difference in (4.69):

$$
\begin{aligned}
& \frac{v^{n+1}-v^{n}}{\Delta t}=\frac{1}{m}\left(F\left(t_{n}\right)-s\left(u^{n}\right)-f\left(v^{n}\right)\right), \\
& \frac{u^{n+1}-u^{n}}{\Delta t}=v^{n+1},
\end{aligned}
$$

We can easily solve for the new unknowns $v^{n+1}$ and $u^{n+1}$ :

$$
\begin{aligned}
& v^{n+1}=v^{n}+\frac{\Delta t}{m}\left(F\left(t_{n}\right)-s\left(u^{n}\right)-f\left(v^{n}\right)\right), \\
& u^{n+1}=u^{n}+\Delta t v^{n+1} .
\end{aligned}
$$




\section{Remark on the ordering of the ODEs}

The ordering of the ODEs in the ODE system is important for the extended model (4.68)-(4.69). Imagine that we write the equation for $u^{\prime}$ first and then the one for $v^{\prime}$. The Euler-Cromer method would then first use a forward difference for $u^{n+1}$ and then a backward difference for $v^{n+1}$. The latter would lead to a nonlinear algebraic equation for $v^{n+1}$,

$$
v^{n+1}+\frac{\Delta t}{m} f\left(v^{n+1}\right)=v^{n}+\frac{\Delta t}{m}\left(F\left(t_{n+1}\right)-s\left(u^{n+1}\right)\right),
$$

if $f(v)$ is a nonlinear function of $v$. This would require a numerical method for nonlinear algebraic equations to find $v^{n+1}$, while updating $v^{n+1}$ through a forward difference gives an equation for $v^{n+1}$ that is linear and trivial to solve by hand.

We can implement the Euler-Cromer method like this:

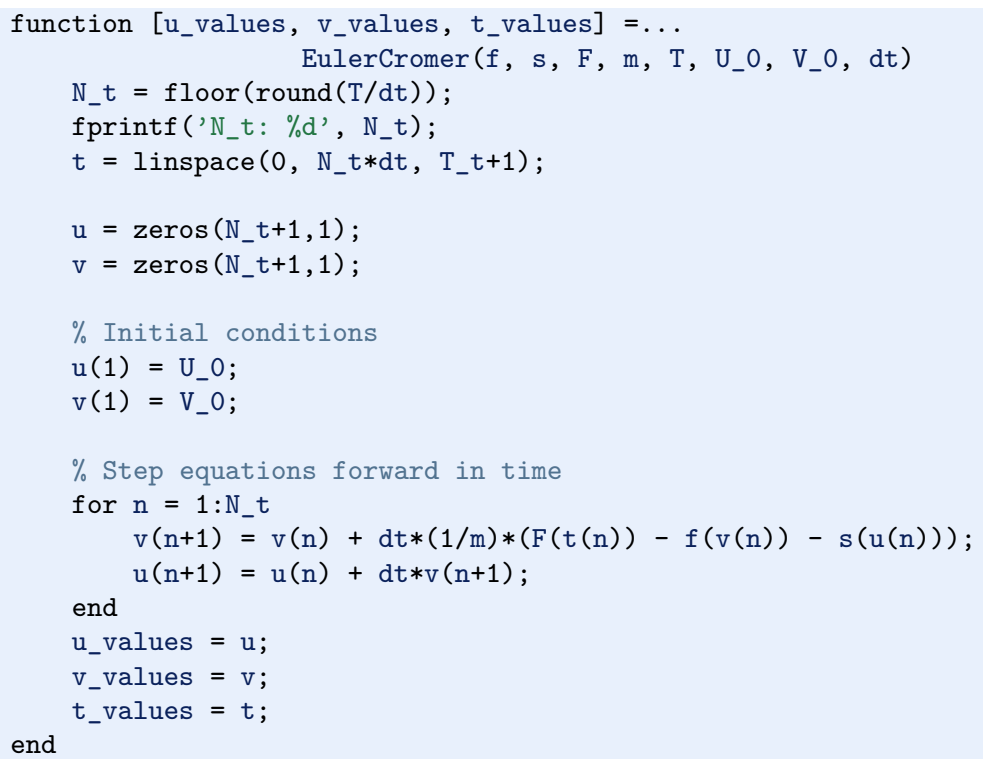

The 4-th order Runge-Kutta method The RK4 method just evaluates the righthand side of the ODE system,

$$
\left(\frac{1}{m}(F(t)-s(u)-f(v)), v\right)
$$

for known values of $u, v$, and $t$, so the method is very simple to use regardless of how the functions $s(u)$ and $f(v)$ are chosen. 


\subsubsection{Illustration of Linear Damping}

We consider an engineering system with a linear spring, $s(u)=k x$, and a viscous damper, where the damping force is proportional to $u^{\prime}, f\left(u^{\prime}\right)=b u^{\prime}$, for some constant $b>0$. This choice may model the vertical spring system in a car (but engineers often like to illustrate such a system by a horizontal moving mass like the one depicted in Fig. 4.25). We may choose simple values for the constants to illustrate basic effects of damping (and later excitations). Choosing the oscillations to be the simple $u(t)=\cos t$ function in the undamped case, we may set $m=1$, $k=1, b=0.3, U_{0}=1, V_{0}=0$. The following function implements this case:

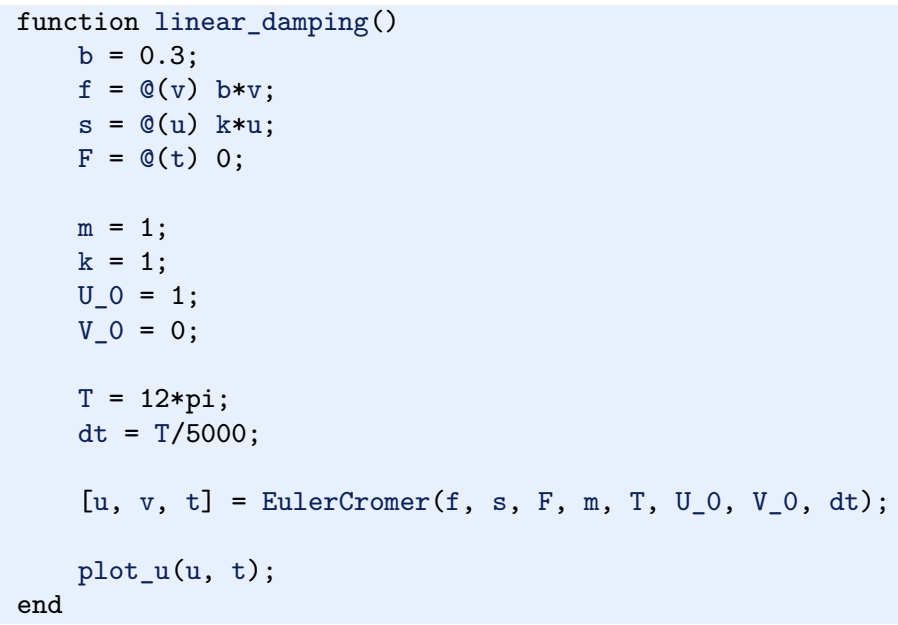

The plot_u function is a collection of plot statements for plotting $u(t)$, or a part of it. Figure 4.27 shows the effect of the $b u^{\prime}$ term: we have oscillations with (an approximate) period $2 \pi$, as expected, but the amplitude is efficiently damped.

\section{Remark about working with a scaled problem}

Instead of setting $b=0.3$ and $m=k=U_{0}=1$ as fairly "unlikely" physical values, it would be better to scale the equation $m u^{\prime \prime}+b u^{\prime}+k u=0$. This means that we introduce dimensionless independent and dependent variables:

$$
\bar{t}=\frac{t}{t_{c}}, \quad \bar{u}=\frac{u}{u_{c}},
$$

where $t_{c}$ and $u_{c}$ are characteristic sizes of time and displacement, respectively, such that $\bar{t}$ and $\bar{u}$ have their typical size around unity. In the present problem, we can choose $u_{c}=U_{0}$ and $t_{c}=\sqrt{m / k}$. This gives the following scaled (or dimensionless) problem for the dimensionless quantity $\bar{u}(\bar{t})$ :

$$
\frac{d^{2} \bar{u}}{d \bar{t}^{2}}+\beta \frac{d \bar{u}}{d \bar{t}}+\bar{u}=0, \quad \bar{u}(0)=1, \bar{u}^{\prime}(0)=0, \quad \beta=\frac{b}{\sqrt{m k}} .
$$

The striking fact is that there is only one physical parameter in this problem: the dimensionless number $\beta$. Solving this problem corresponds to solving the 


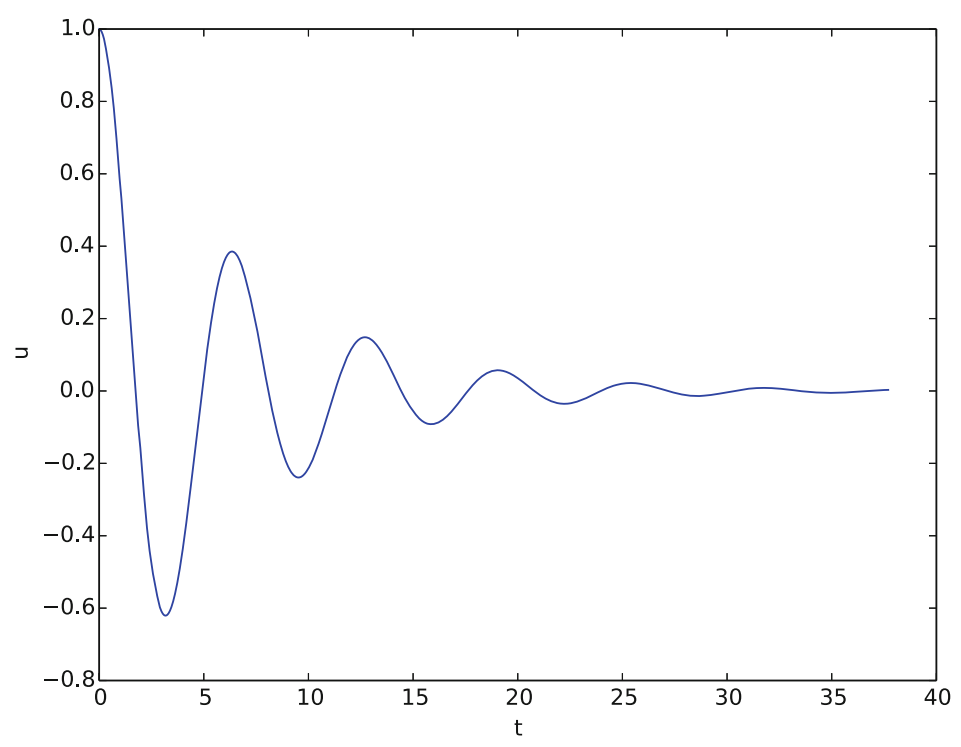

Fig.4.27 Effect of linear damping

original problem (with dimensions) with the parameters $m=k=U_{0}=1$ and $b=\beta$. However, solving the dimensionless problem is more general: if we have a solution $\bar{u}(\bar{t} ; \beta)$, we can find the physical solution of a range of problems since

$$
u(t)=U_{0} \bar{u}(t \sqrt{k / m} ; \beta) .
$$

As long as $\beta$ is fixed, we can find $u$ for any $U_{0}, k$, and $m$ from the above formula! In this way, a time consuming simulation can be done only once, but still provide many solutions. This demonstrates the power of working with scaled or dimensionless problems.

\subsubsection{Illustration of Linear Damping with Sinusoidal Excitation}

We now extend the previous example to also involve some external oscillating force on the system: $F(t)=A \sin (w t)$. Driving a car on a road with sinusoidal bumps might give such an external excitation on the spring system in the car ( $w$ is related to the velocity of the car).

With $A=0.5$ and $w=3$,

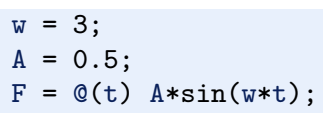

we get the graph in Fig. 4.28. The striking difference from Fig. 4.27 is that the oscillations start out as a damped $\cos t$ signal without much influence of the external force, but then the free oscillations of the undamped system $(\cos t) u^{\prime \prime}+u=0$ 


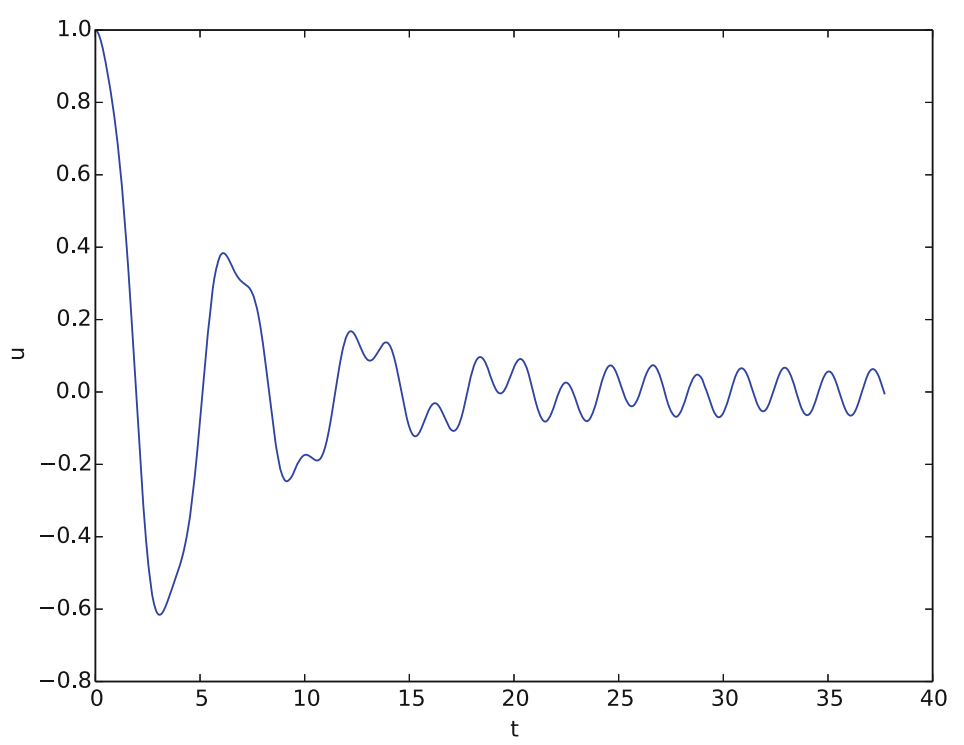

Fig. 4.28 Effect of linear damping in combination with a sinusoidal external force

die out and the external force $0.5 \sin (3 t)$ induces oscillations with a shorter period $2 \pi / 3$. You are encouraged to play around with a larger $A$ and switch from a sine to a cosine in $F$ and observe the effects. If you look this up in a physics book, you can find exact analytical solutions to the differential equation problem in these cases.

A particularly interesting case arises when the excitation force has the same frequency as the free oscillations of the undamped system, i.e., $F(t)=A \sin t$. With the same amplitude $A=0.5$, but a smaller damping $b=0.1$, the oscillations in Fig. 4.28 becomes qualitatively very different as the amplitude grows significantly larger over some periods. This phenomenon is called resonance and is exemplified in Fig. 4.29. Removing the damping results in an amplitude that grows linearly in time.

\subsubsection{Spring-Mass System with Sliding Friction}

A body with mass $m$ is attached to a spring with stiffness $k$ while sliding on a plane surface. The body is also subject to a friction force $f\left(u^{\prime}\right)$ due to the contact between the body and the plane. Figure 4.30 depicts the situation. The friction force $f\left(u^{\prime}\right)$ can be modeled by Coulomb friction:

$$
f\left(u^{\prime}\right)= \begin{cases}-\mu m g, & u^{\prime}<0 \\ \mu m g, & u^{\prime}>0 \\ 0, & u^{\prime}=0\end{cases}
$$




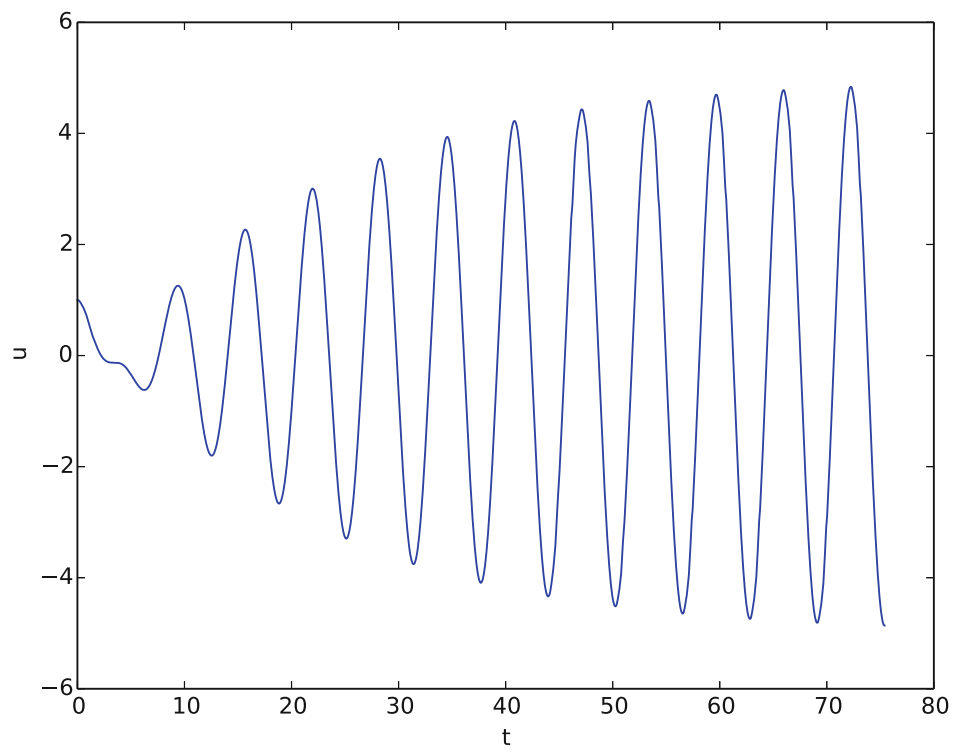

Fig.4.29 Excitation force that causes resonance

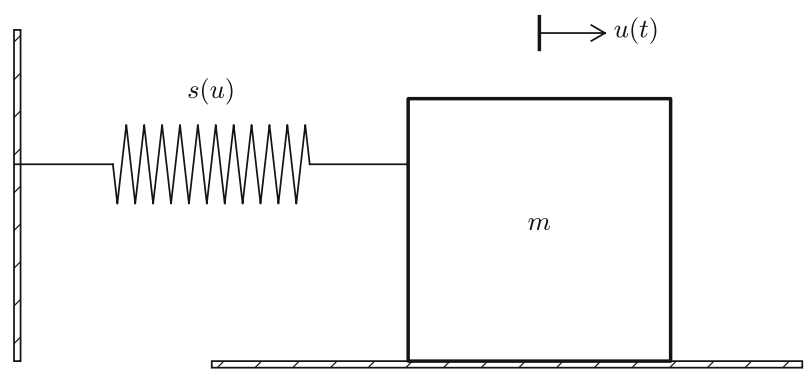

Fig. 4.30 Sketch of a one-dimensional, oscillating dynamic system subject to sliding friction and a spring force

where $\mu$ is the friction coefficient, and $m g$ is the normal force on the surface where the body slides. This formula can also be written as $f\left(u^{\prime}\right)=\mu m g \operatorname{sign}\left(u^{\prime}\right)$, provided the signum function $\operatorname{sign}(x)$ is defined to be zero for $x=0$ (the sign function in Matlab" has this property). To check that the signs in the definition of $f$ are right, recall that the actual physical force is $-f$ and this is positive (i.e., $f<0$ ) when it works against the body moving with velocity $u^{\prime}<0$.

The nonlinear spring force is taken as

$$
s(u)=-k \alpha^{-1} \tanh (\alpha u)
$$

which is approximately $-k u$ for small $u$, but stabilizes at $\pm k / \alpha$ for large $\pm \alpha u$. Here is a plot with $k=1000$ and $u \in[-0.1,0.1]$ for three $\alpha$ values: 


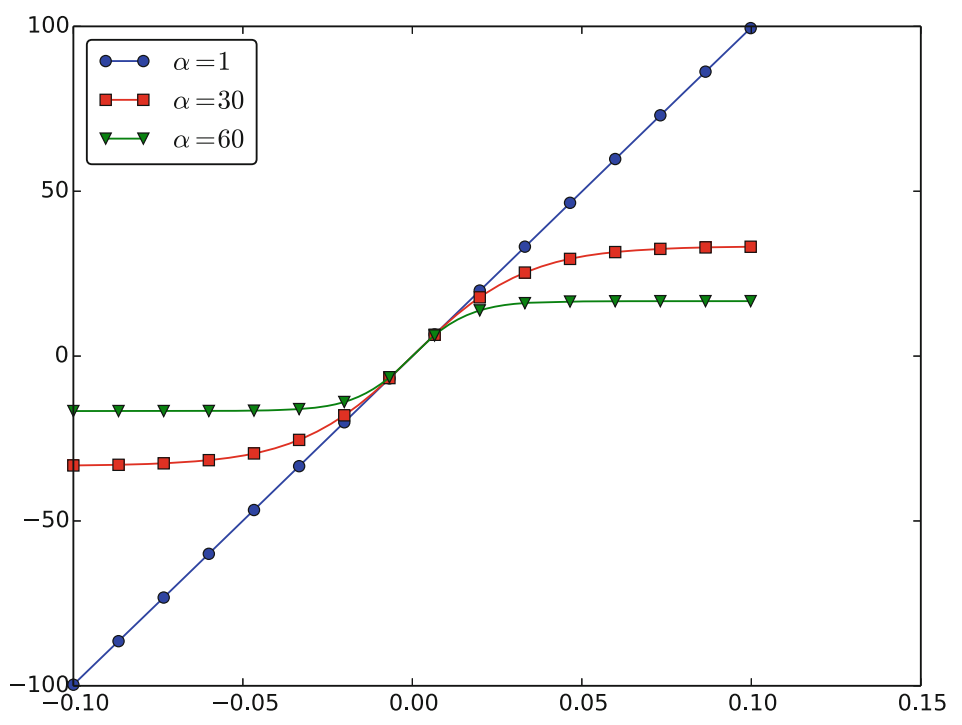

If there is no external excitation force acting on the body, we have the equation of motion

$$
m u^{\prime \prime}+\mu m g \operatorname{sign}\left(u^{\prime}\right)+k \alpha^{-1} \tanh (\alpha u)=0 .
$$

Let us simulate a situation where a body of mass $1 \mathrm{~kg}$ slides on a surface with $\mu=0.4$, while attached to a spring with stiffness $k=1000 \mathrm{~kg} / \mathrm{s}^{2}$. The initial displacement of the body is $10 \mathrm{~cm}$, and the $\alpha$ parameter in $s(u)$ is set to $601 / \mathrm{m}$. Using the EulerCromer function from the EulerCromer code, we can write a function sliding_friction for solving this problem:

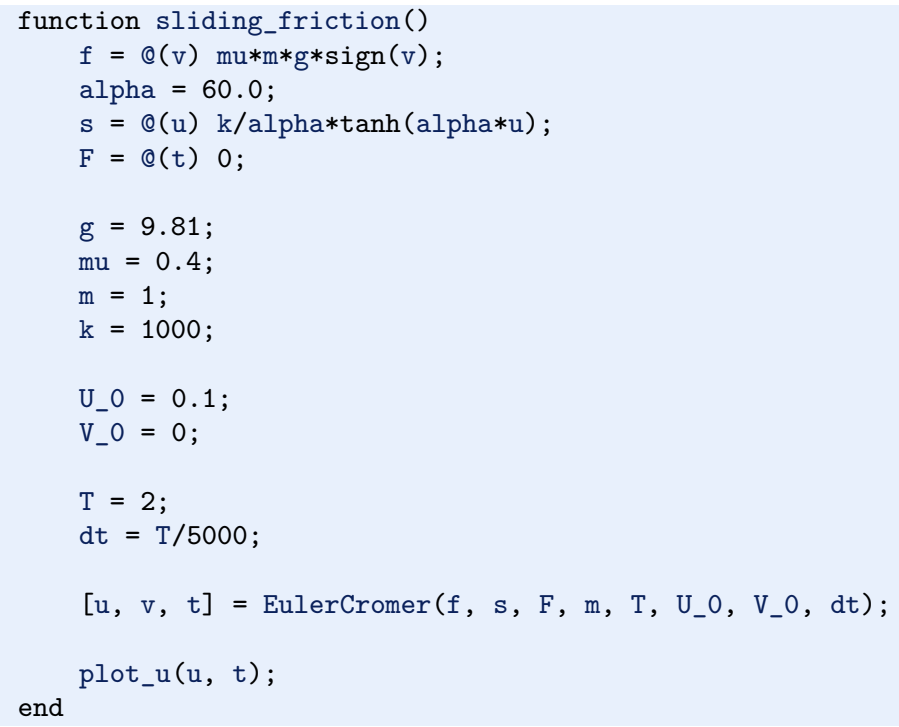

Running the sliding_friction function gives us the results in Fig. 4.31 with $s(u)=k \alpha^{-1} \tanh (\alpha u)$ (left) and the linearized version $s(u)=k u$ (right). 

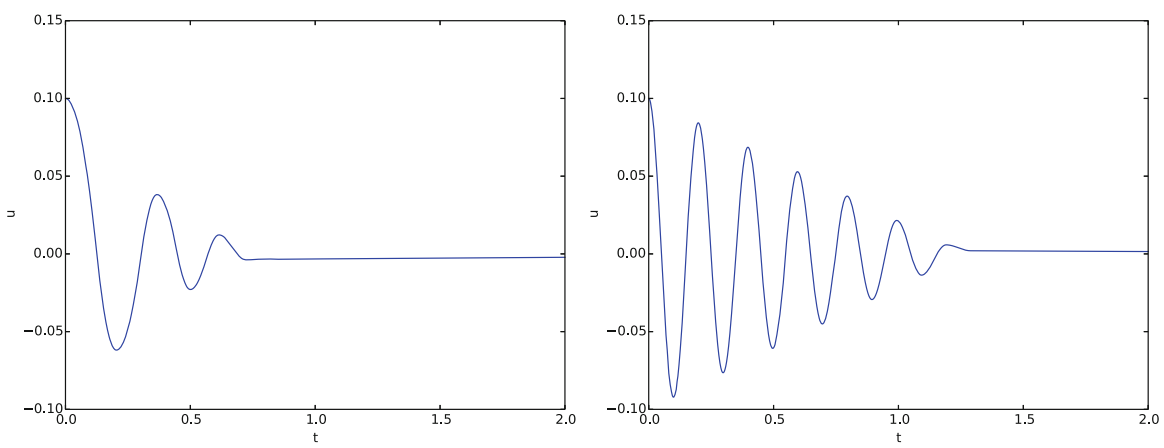

Fig.4.31 Effect of nonlinear (left) and linear (right) spring on sliding friction

\subsubsection{A Finite Difference Method; Undamped, Linear Case}

We shall now address numerical methods for the second-order ODE

$$
u^{\prime \prime}+\omega^{2} u=0, \quad u(0)=U_{0}, u^{\prime}(0)=0, t \in(0, T]
$$

without rewriting the ODE as a system of first-order ODEs. The primary motivation for "yet another solution method" is that the discretization principles result in a very good scheme, and more importantly, the thinking around the discretization can be reused when solving partial differential equations.

The main idea of this numerical method is to approximate the second-order derivative $u^{\prime \prime}$ by a finite difference. While there are several choices of difference approximations to first-order derivatives, there is one dominating formula for the second-order derivative:

$$
u^{\prime \prime}\left(t_{n}\right) \approx \frac{u^{n+1}-2 u^{n}+u^{n-1}}{\Delta t^{2}} .
$$

The error in this approximation is proportional to $\Delta t^{2}$. Letting the ODE be valid at some arbitrary time point $t_{n}$,

$$
u^{\prime \prime}\left(t_{n}\right)+\omega^{2} u\left(t_{n}\right)=0,
$$

we just insert the approximation (4.74) to get

$$
\frac{u^{n+1}-2 u^{n}+u^{n-1}}{\Delta t^{2}}=-\omega^{2} u^{n} .
$$

We now assume that $u^{n-1}$ and $u^{n}$ are already computed and that $u^{n+1}$ is the new unknown. Solving with respect to $u^{n+1}$ gives

$$
u^{n+1}=2 u^{n}-u^{n-1}-\Delta t^{2} \omega^{2} u^{n} .
$$

A major problem arises when we want to start the scheme. We know that $u^{0}=$ $U_{0}$, but applying (4.76) for $n=0$ to compute $u^{1}$ leads to

$$
u^{1}=2 u^{0}-u^{-1}-\Delta t^{2} \omega^{2} u^{0},
$$


where we do not know $u^{-1}$. The initial condition $u^{\prime}(0)=0$ can help us to eliminate $u^{-1}-$ and this condition must anyway be incorporated in some way. To this end, we discretize $u^{\prime}(0)=0$ by a centered difference,

$$
u^{\prime}(0) \approx \frac{u^{1}-u^{-1}}{2 \Delta t}=0 .
$$

It follows that $u^{-1}=u^{1}$, and we can use this relation to eliminate $u^{-1}$ in (4.77):

$$
u^{1}=u^{0}-\frac{1}{2} \Delta t^{2} \omega^{2} u^{0}
$$

With $u^{0}=U_{0}$ and $u^{1}$ computed from (4.78), we can compute $u^{2}, u^{3}$, and so forth from (4.76). Exercise 4.19 asks you to explore how the steps above are modified in case we have a nonzero initial condition $u^{\prime}(0)=V_{0}$.

Remark on a simpler method for computing $u^{1}$

We could approximate the initial condition $u^{\prime}(0)$ by a forward difference:

$$
u^{\prime}(0) \approx \frac{u^{1}-u^{0}}{\Delta t}=0
$$

leading to $u^{1}=u^{0}$. Then we can use (4.76) for the coming time steps. However, this forward difference has an error proportional to $\Delta t$, while the centered difference we used has an error proportional to $\Delta t^{2}$, which is compatible with the accuracy (error goes like $\Delta t^{2}$ ) used in the discretization of the differential equation.

The method for the second-order ODE described above goes under the name Störmer's method or Verlet integration ${ }^{7}$. It turns out that this method is mathematically equivalent with the Euler-Cromer scheme (!). Or more precisely, the general formula (4.76) is equivalent with the Euler-Cromer formula, but the scheme for the first time level (4.78) implements the initial condition $u^{\prime}(0)$ slightly more accurately than what is naturally done in the Euler-Cromer scheme. The latter will do

$$
v^{1}=v^{0}-\Delta t \omega^{2} u^{0}, \quad u^{1}=u^{0}+\Delta t v^{1}=u^{0}-\Delta t^{2} \omega^{2} u^{0},
$$

which differs from $u^{1}$ in (4.78) by an amount $\frac{1}{2} \Delta t^{2} \omega^{2} u^{0}$.

Because of the equivalence of (4.76) with the Euler-Cromer scheme, the numerical results will have the same nice properties such as a constant amplitude. There will be a phase error as in the Euler-Cromer scheme, but this error is effectively reduced by reducing $\Delta t$, as already demonstrated.

The implementation of (4.78) and (4.76) is straightforward in a function (file osc_2nd_order.m):

\footnotetext{
${ }^{7}$ http://en.wikipedia.org/wiki/Verlet_integration
} 


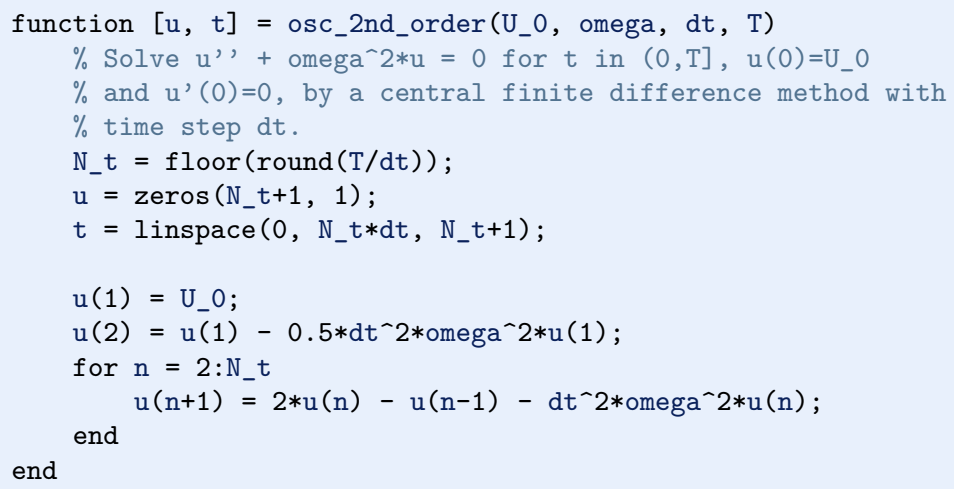

\subsubsection{A Finite Difference Method; Linear Damping}

A key issue is how to generalize the scheme from Sect. 4.3.12 to a differential equation with more terms. We start with the case of a linear damping term $f\left(u^{\prime}\right)=$ $b u^{\prime}$, a possibly nonlinear spring force $s(u)$, and an excitation force $F(t)$ :

$$
m u^{\prime \prime}+b u^{\prime}+s(u)=F(t), \quad u(0)=U_{0}, u^{\prime}(0)=0, t \in(0, T] .
$$

We need to find the appropriate difference approximation to $u^{\prime}$ in the $b u^{\prime}$ term. A good choice is the centered difference

$$
u^{\prime}\left(t_{n}\right) \approx \frac{u^{n+1}-u^{n-1}}{2 \Delta t}
$$

Sampling the equation at a time point $t_{n}$,

$$
m u^{\prime \prime}\left(t_{n}\right)+b u^{\prime}\left(t_{n}\right)+s\left(u^{n}\right)=F\left(t_{n}\right),
$$

and inserting the finite difference approximations to $u^{\prime \prime}$ and $u^{\prime}$ results in

$$
m \frac{u^{n+1}-2 u^{n}+u^{n-1}}{\Delta t^{2}}+b \frac{u^{n+1}-u^{n-1}}{2 \Delta t}+s\left(u^{n}\right)=F^{n},
$$

where $F^{n}$ is a short notation for $F\left(t_{n}\right)$. Equation (4.81) is linear in the unknown $u^{n+1}$, so we can easily solve for this quantity:

$$
u^{n+1}=\left(2 m u^{n}+\left(\frac{b}{2} \Delta t-m\right) u^{n-1}+\Delta t^{2}\left(F^{n}-s\left(u^{n}\right)\right)\right)\left(m+\frac{b}{2} \Delta t\right)^{-1} .
$$

As in the case without damping, we need to derive a special formula for $u^{1}$. The initial condition $u^{\prime}(0)=0$ implies also now that $u^{-1}=u^{1}$, and with (4.82) for $n=0$, we get

$$
u^{1}=u^{0}+\frac{\Delta t^{2}}{2 m}\left(F^{0}-s\left(u^{0}\right)\right) .
$$


In the more general case with a nonlinear damping term $f\left(u^{\prime}\right)$,

$$
m u^{\prime \prime}+f\left(u^{\prime}\right)+s(u)=F(t),
$$

we get

$$
m \frac{u^{n+1}-2 u^{n}+u^{n-1}}{\Delta t^{2}}+f\left(\frac{u^{n+1}-u^{n-1}}{2 \Delta t}\right)+s\left(u^{n}\right)=F^{n}
$$

which is a nonlinear algebraic equation for $u^{n+1}$ that must be solved by numerical methods. A much more convenient scheme arises from using a backward difference for $u^{\prime}$,

$$
u^{\prime}\left(t_{n}\right) \approx \frac{u^{n}-u^{n-1}}{\Delta t},
$$

because the damping term will then be known, involving only $u^{n}$ and $u^{n-1}$, and we can easily solve for $u^{n+1}$.

The downside of the backward difference compared to the centered difference (4.80) is that it reduces the order of the accuracy in the overall scheme from $\Delta t^{2}$ to $\Delta t$. In fact, the Euler-Cromer scheme evaluates a nonlinear damping term as $f\left(v^{n}\right)$ when computing $v^{n+1}$, and this is equivalent to using the backward difference above. Consequently, the convenience of the Euler-Cromer scheme for nonlinear damping comes at a cost of lowering the overall accuracy of the scheme from second to first order in $\Delta t$. Using the same trick in the finite difference scheme for the second-order differential equation, i.e., using the backward difference in $f\left(u^{\prime}\right)$, makes this scheme equally convenient and accurate as the Euler-Cromer scheme in the general nonlinear case $m u^{\prime \prime}+f\left(u^{\prime}\right)+s(u)=F$.

\subsection{Exercises}

\section{Exercise 4.1: Geometric construction of the Forward Euler method}

Section 4.1.4 describes a geometric interpretation of the Forward Euler method. This exercise will demonstrate the geometric construction of the solution in detail. Consider the differential equation $u^{\prime}=u$ with $u(0)=1$. We use time steps $\Delta t=1$.

a) Start at $t=0$ and draw a straight line with slope $u^{\prime}(0)=u(0)=1$. Go one time step forward to $t=\Delta t$ and mark the solution point on the line.

b) Draw a straight line through the solution point $\left(\Delta t, u^{1}\right)$ with slope $u^{\prime}(\Delta t)=u^{1}$. Go one time step forward to $t=2 \Delta t$ and mark the solution point on the line.

c) Draw a straight line through the solution point $\left(2 \Delta t, u^{2}\right)$ with slope $u^{\prime}(2 \Delta t)=$ $u^{2}$. Go one time step forward to $t=3 \Delta t$ and mark the solution point on the line.

d) Set up the Forward Euler scheme for the problem $u^{\prime}=u$. Calculate $u^{1}, u^{2}$, and $u^{3}$. Check that the numbers are the same as obtained in a)-c).

Filename: ForwardEuler_geometric_solution.m. 


\section{Exercise 4.2: Make test functions for the Forward Euler method}

The purpose of this exercise is to make a file test_ode_FE.m that makes use of the ode_FE function in the file ode_FE. $m$ and automatically verifies the implementation of ode_FE.

a) The solution computed by hand in Exercise 4.1 can be used as a reference solution. Make a function test_ode_FE_1() that calls ode_FE to compute three time steps in the problem $u^{\prime}=u, u(0)=1$, and compare the three values $u^{1}$, $u^{2}$, and $u^{3}$ with the values obtained in Exercise 4.1.

b) The test in a) can be made more general using the fact that if $f$ is linear in $u$ and does not depend on $t$, i.e., we have $u^{\prime}=r u$, for some constant $r$, the Forward Euler method has a closed form solution as outlined in Sect. 4.1.1: $u^{n}=U_{0}(1+$ $r \Delta t)^{n}$. Use this result to construct a test function test_ode_FE_2() that runs a number of steps in ode_FE and compares the computed solution with the listed formula for $u^{n}$.

Filename: test_ode_FE.m.

\section{Exercise 4.3: Implement and evaluate Heun's method}

a) A 2nd-order Runge-Kutta method, also known has Heun's method, is derived in Sect. 4.3.5. Make a function ode_Heun ( $f, U_{-} 0$, dt, T) (as a counterpart to ode_FE(f, U_o, dt, T) in ode_FE.m) for solving a scalar ODE problem $u^{\prime}=f(u, t), u(0)=U_{0}, t \in(0, T]$, with this method using a time step size $\Delta t$.

b) Solve the simple ODE problem $u^{\prime}=u, u(0)=1$, by the ode_Heun and the ode_FE function. Make a plot that compares Heun's method and the Forward Euler method with the exact solution $u(t)=e^{t}$ for $t \in[0,6]$. Use a time step $\Delta t=0.5$.

c) For the case in b), find through experimentation the largest value of $\Delta t$ where the exact solution and the numerical solution by Heun's method cannot be distinguished visually. It is of interest to see how far off the curve the Forward Euler method is when Heun's method can be regarded as "exact" (for visual purposes).

Filename: ode_Heun.m.

\section{Exercise 4.4: Find an appropriate time step; logistic model}

Compute the numerical solution of the logistic equation for a set of repeatedly halved time steps: $\Delta t_{k}=2^{-k} \Delta t, k=0,1, \ldots$. Plot the solutions corresponding to the last two time steps $\Delta t_{k}$ and $\Delta t_{k-1}$ in the same plot. Continue doing this until you cannot visually distinguish the two curves in the plot. Then one has found a sufficiently small time step.

Hint Extend the logistic.m file. Introduce a loop over $k$, write out $\Delta t_{k}$, and ask the user if the loop is to be continued.

Filename: logistic_dt.m. 


\section{Exercise 4.5: Find an appropriate time step; SIR model}

Repeat Exercise 4.4 for the SIR model.

Hint Use the ode_FE function and make a modified demo_SIR function that has a loop over repeatedly halved time steps. Plot $S, I$, and $R$ versus time for the two last time step sizes in the same plot.

Filename: SIR_dt.m.

\section{Exercise 4.6: Model an adaptive vaccination campaign}

In the SIRV model with time-dependent vaccination from Sect. 4.2.9, we want to test the effect of an adaptive vaccination campaign where vaccination is offered as long as half of the population is not vaccinated. The campaign starts after $\Delta$ days. That is, $p=p_{0}$ if $V<\frac{1}{2}\left(S^{0}+I^{0}\right)$ and $t>\Delta$ days, otherwise $p=0$.

Demonstrate the effect of this vaccination policy: choose $\beta, \gamma$, and $v$ as in Sect. 4.2.9, set $p=0.001, \Delta=10$ days, and simulate for 200 days.

Hint This discontinuous $p(t)$ function is easiest implemented as a Matlab function containing the indicated if test. You may use the file SIRV1.m as starting point, but note that it implements a time-dependent $p(t)$ via an array.

Filename: SIRV_p_adapt.m.

\section{Exercise 4.7: Make a SIRV model with time-limited effect of vaccination}

We consider the SIRV model from Sect. 4.2.8, but now the effect of vaccination is time-limited. After a characteristic period of time, $\pi$, the vaccination is no more effective and individuals are consequently moved from the $\mathrm{V}$ to the $\mathrm{S}$ category and can be infected again. Mathematically, this can be modeled as an average leakage $-\pi^{-1} V$ from the $\mathrm{V}$ category to the $\mathrm{S}$ category (i.e., a gain $\pi^{-1} V$ in the latter). Write up the complete model, implement it, and rerun the case from Sect. 4.2.8 with various choices of parameters to illustrate various effects.

Filename: SIRV1_V2S.m.

\section{Exercise 4.8: Refactor a flat program}

Consider the file osc_FE.m implementing the Forward Euler method for the oscillating system model (4.43)-(4.44). The osc_FE.m is what we often refer to as a flat program, meaning that it is just one main program with no functions. To easily reuse the numerical computations in other contexts, place the part that produces the numerical solution (allocation of arrays, initializing the arrays at time zero, and the time loop) in a function osc_FE (X_O , omega, dt, T), which returns $u, v$, t. Place the particular computational example in osc_FE.m in a function demo(). Construct the file osc_FE_func.m such that the osc_FE function can easily be reused in other programs.

Filename: osc_FE_func.m.

\section{Exercise 4.9: Simulate oscillations by a general ODE solver}

Solve the system (4.43)-(4.44) using the general solver ode_FE described in Sect. 4.2.6. Program the ODE system and the call to the ode_FE function in a separate file osc_ode_FE.m. 
Equip this file with a test function that reads a file with correct $u$ values and compares these with those computed by the ode_FE function. To find correct $u$ values, modify the program osc_FE.m to dump the $u$ array to file, run osc_FE.m, and let the test function read the reference results from that file.

Filename: osc_ode_FE.m.

\section{Exercise 4.10: Compute the energy in oscillations}

a) Make a function osc_energy ( $\mathrm{u}, \mathrm{v}$, omega) for returning the potential and kinetic energy of an oscillating system described by (4.43)-(4.44). The potential energy is taken as $\frac{1}{2} \omega^{2} u^{2}$ while the kinetic energy is $\frac{1}{2} v^{2}$. (Note that these expressions are not exactly the physical potential and kinetic energy, since these would be $\frac{1}{2} m v^{2}$ and $\frac{1}{2} k u^{2}$ for a model $m x^{\prime \prime}+k x=0$.)

Place the osc_energy in a separate file osc_energy.m such that the function can be called from other functions.

b) Add a call to osc_energy in the programs osc_FE.m and osc_EC.m and plot the sum of the kinetic and potential energy. How does the total energy develop for the Forward Euler and the Euler-Cromer schemes?

Filenames: osc_energy.m,osc_FE_energy.m,osc_EC_energy.m.

\section{Exercise 4.11: Use a Backward Euler scheme for population growth}

We consider the ODE problem $N^{\prime}(t)=r N(t), N(0)=N_{0}$. At some time, $t_{n}=n \Delta t$, we can approximate the derivative $N^{\prime}\left(t_{n}\right)$ by a backward difference, see Fig. 4.19:

$$
N^{\prime}\left(t_{n}\right) \approx \frac{N\left(t_{n}\right)-N\left(t_{n}-\Delta t\right)}{\Delta t}=\frac{N^{n}-N^{n-1}}{\Delta t}
$$

which leads to

$$
\frac{N^{n}-N^{n-1}}{\Delta t}=r N^{n}
$$

called the Backward Euler scheme.

a) Find an expression for the $N^{n}$ in terms of $N^{n-1}$ and formulate an algorithm for computing $N^{n}, n=1,2, \ldots, N_{t}$.

b) Implement the algorithm in a) in a function growth_BE (N_0, dt, T) for solving $N^{\prime}=r N, N(0)=N_{0}, t \in(0, T]$, with time step $\Delta t(\mathrm{dt})$.

c) Implement the Forward Euler scheme in a function growth_FE (N_0, dt, T) as described in b).

d) Compare visually the solution produced by the Forward and Backward Euler schemes with the exact solution when $r=1$ and $T=6$. Make two plots, one with $\Delta t=0.5$ and one with $\Delta t=0.05$.

Filename: growth_BE.m. 


\section{Exercise 4.12: Use a Crank-Nicolson scheme for population growth}

It is recommended to do Exercise 4.11 prior to the present one. Here we look at the same population growth model $N^{\prime}(t)=r N(t), N(0)=N_{0}$. The time derivative $N^{\prime}(t)$ can be approximated by various types of finite differences. Exercise 4.11 considers a backward difference (Fig. 4.19), while Sect. 4.1.2 explained the forward difference (Fig. 4.2). A centered difference is more accurate than a backward or forward difference:

$$
N^{\prime}\left(t_{n}+\frac{1}{2} \Delta t\right) \approx \frac{N\left(t_{n}+\Delta t\right)-N\left(t_{n}\right)}{\Delta t}=\frac{N^{n+1}-N^{n}}{\Delta t} .
$$

This type of difference, applied at the point $t_{n+\frac{1}{2}}=t_{n}+\frac{1}{2} \Delta t$, is illustrated geometrically in Fig. 4.20.

a) Insert the finite difference approximation in the ODE $N^{\prime}=r N$ and solve for the unknown $N^{n+1}$, assuming $N^{n}$ is already computed and hence known. The resulting computational scheme is often referred to as a Crank-Nicolson scheme.

b) Implement the algorithm in a) in a function growth_CN (N_O, dt, T) for solving $N^{\prime}=r N, N(0)=N_{0}, t \in(0, T]$, with time step $\Delta t(\mathrm{dt})$.

c) Make plots for comparing the Crank-Nicolson scheme with the Forward and Backward Euler schemes in the same test problem as in Exercise 4.11.

Filename: growth_CN.m.

\section{Exercise 4.13: Understand finite differences via Taylor series}

The Taylor series around a point $x=a$ can for a function $f(x)$ be written

$$
\begin{aligned}
f(x)= & f(a)+\frac{d}{d x} f(a)(x-a)+\frac{1}{2 !} \frac{d^{2}}{d x^{2}} f(a)(x-a)^{2} \\
& +\frac{1}{3 !} \frac{d^{3}}{d x^{3}} f(a)(x-a)^{3}+\ldots \\
= & \sum_{i=0}^{\infty} \frac{1}{i !} \frac{d^{i}}{d x^{i}} f(a)(x-a)^{i} .
\end{aligned}
$$

For a function of time, as addressed in our ODE problems, we would use $u$ instead of $f, t$ instead of $x$, and a time point $t_{n}$ instead of $a$ :

$$
\begin{aligned}
u(t)= & u\left(t_{n}\right)+\frac{d}{d t} u\left(t_{n}\right)\left(t-t_{n}\right)+\frac{1}{2 !} \frac{d^{2}}{d t^{2}} u\left(t_{n}\right)\left(t-t_{n}\right)^{2} \\
& +\frac{1}{3 !} \frac{d^{3}}{d t^{3}} u\left(t_{n}\right)\left(t-t_{n}\right)^{3}+\ldots \\
= & \sum_{i=0}^{\infty} \frac{1}{i !} \frac{d^{i}}{d t^{i}} u\left(t_{n}\right)\left(t-t_{n}\right)^{i} .
\end{aligned}
$$

a) A forward finite difference approximation to the derivative $f^{\prime}(a)$ reads

$$
u^{\prime}\left(t_{n}\right) \approx \frac{u\left(t_{n}+\Delta t\right)-u\left(t_{n}\right)}{\Delta t} .
$$


We can justify this formula mathematically through Taylor series. Write up the Taylor series for $u\left(t_{n}+\Delta t\right)$ (around $t=t_{n}$, as given above), and then solve the expression with respect to $u^{\prime}\left(t_{n}\right)$. Identify, on the right-hand side, the finite difference approximation and an infinite series. This series is then the error in the finite difference approximation. If $\Delta t$ is assumed small (i.e. $\Delta t<<1$ ), $\Delta t$ will be much larger than $\Delta t^{2}$, which will be much larger than $\Delta t^{3}$, and so on. The leading order term in the series for the error, i.e., the error with the least power of $\Delta t$ is a good approximation of the error. Identify this term.

b) Repeat a) for a backward difference:

$$
u^{\prime}\left(t_{n}\right) \approx \frac{u\left(t_{n}\right)-u\left(t_{n}-\Delta t\right)}{\Delta t} .
$$

This time, write up the Taylor series for $u\left(t_{n}-\Delta t\right)$ around $t_{n}$. Solve with respect to $u^{\prime}\left(t_{n}\right)$, and identify the leading order term in the error. How is the error compared to the forward difference?

c) A centered difference approximation to the derivative, as explored in Exercise 4.12 , can be written

$$
u^{\prime}\left(t_{n}+\frac{1}{2} \Delta t\right) \approx \frac{u\left(t_{n}+\Delta t\right)-u\left(t_{n}\right)}{\Delta t} .
$$

Write up the Taylor series for $u\left(t_{n}\right)$ around $t_{n}+\frac{1}{2} \Delta t$ and the Taylor series for $u\left(t_{n}+\Delta t\right)$ around $t_{n}+\frac{1}{2} \Delta t$. Subtract the two series, solve with respect to $u^{\prime}\left(t_{n}+\frac{1}{2} \Delta t\right)$, identify the finite difference approximation and the error terms on the right-hand side, and write up the leading order error term. How is this term compared to the ones for the forward and backward differences?

d) Can you use the leading order error terms in a)-c) to explain the visual observations in the numerical experiment in Exercise 4.12?

e) Find the leading order error term in the following standard finite difference approximation to the second-order derivative:

$$
u^{\prime \prime}\left(t_{n}\right) \approx \frac{u\left(t_{n}+\Delta t\right)-2 u\left(t_{n}\right)+u\left(t_{n}-\Delta t\right)}{\Delta t} .
$$

Hint Express $u\left(t_{n} \pm \Delta t\right)$ via Taylor series and insert them in the difference formula. Filename: Taylor_differences.pdf.

\section{Exercise 4.14: Use a Backward Euler scheme for oscillations}

Consider (4.43)-(4.44) modeling an oscillating engineering system. This $2 \times 2$ ODE system can be solved by the Backward Euler scheme, which is based on discretizing derivatives by collecting information backward in time. More specifically, $u^{\prime}(t)$ is approximated as

$$
u^{\prime}(t) \approx \frac{u(t)-u(t-\Delta t)}{\Delta t} .
$$

A general vector ODE $u^{\prime}=f(u, t)$, where $u$ and $f$ are vectors, can use this approximation as follows:

$$
\frac{u^{n}-u^{n-1}}{\Delta t}=f\left(u^{n}, t_{n}\right)
$$


which leads to an equation for the new value $u^{n}$ :

$$
u^{n}-\Delta t f\left(u^{n}, t_{n}\right)=u^{n-1}
$$

For a general $f$, this is a system of nonlinear algebraic equations.

However, the ODE (4.43)-(4.44) is linear, so a Backward Euler scheme leads to a system of two algebraic equations for two unknowns:

$$
\begin{aligned}
u^{n}-\Delta t v^{n} & =u^{n-1}, \\
v^{n}+\Delta t \omega^{2} u^{n} & =v^{n-1} .
\end{aligned}
$$

a) Solve the system for $u^{n}$ and $v^{n}$.

b) Implement the found formulas for $u^{n}$ and $v^{n}$ in a program for computing the entire numerical solution of (4.43)-(4.44).

c) Run the program with a $\Delta t$ corresponding to 20 time steps per period of the oscillations (see Sect. 4.3.3 for how to find such a $\Delta t$ ). What do you observe? Increase to 2000 time steps per period. How much does this improve the solution?

Filename: osc_BE.m.

Remarks While the Forward Euler method applied to oscillation problems $u^{\prime \prime}+$ $\omega^{2} u=0$ gives growing amplitudes, the Backward Euler method leads to significantly damped amplitudes.

\section{Exercise 4.15: Use Heun's method for the SIR model}

Make a program that computes the solution of the SIR model from Sect. 4.2.1 both by the Forward Euler method and by Heun's method (or equivalently: the 2nd-order Runge-Kutta method) from Sect. 4.3.5. Compare the two methods in the simulation case from Sect. 4.2.3. Make two comparison plots, one for a large and one for a small time step. Experiment to find what "large" and "small" should be: the large one gives significant differences, while the small one lead to very similar curves. Filename: SIR_Heun.m.

\section{Exercise 4.16: Use Odespy to solve a simple ODE}

Solve

$$
u^{\prime}=-a u+b, \quad u(0)=U_{0}, \quad t \in(0, T]
$$

by the Odespy software. Let the problem parameters $a$ and $b$ be arguments to the right-hand side function that specifies the ODE to be solved. Plot the solution for the case when $a=2, b=1, T=6 / a$, and we use 100 time intervals in $[0, T]$.

Filename: odespy_demo.m.

\section{Exercise 4.17: Set up a Backward Euler scheme for oscillations}

Write the ODE $u^{\prime \prime}+\omega^{2} u=0$ as a system of two first-order ODEs and discretize these with backward differences as illustrated in Fig. 4.19. The resulting method is referred to as a Backward Euler scheme. Identify the matrix and right-hand side of 
the linear system that has to be solved at each time level. Implement the method, either from scratch yourself or using Odespy (the name is odespy. BackwardEuler). Demonstrate that contrary to a Forward Euler scheme, the Backward Euler scheme leads to significant non-physical damping. The figure below shows that even with 60 time steps per period, the results after a few periods are useless:

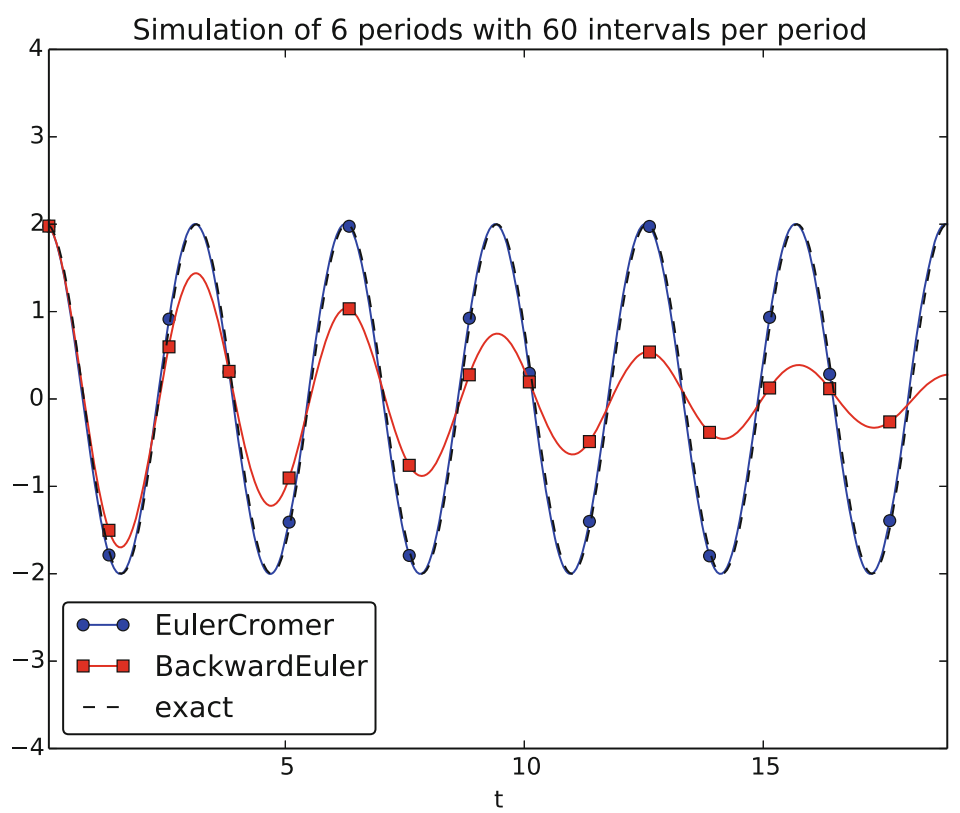

Filename: osc_BE.m.

\section{Exercise 4.18: Set up a Forward Euler scheme for nonlinear and damped oscillations}

Derive a Forward Euler method for the ODE system (4.68)-(4.69). Compare the method with the Euler-Cromer scheme for the sliding friction problem from Sect. 4.3.11:

1. Does the Forward Euler scheme give growing amplitudes?

2. Is the period of oscillation accurate?

3. What is the required time step size for the two methods to have visually coinciding curves?

Filename: osc_FE_general.m.

\section{Exercise 4.19: Discretize an initial condition}

Assume that the initial condition on $u^{\prime}$ is nonzero in the finite difference method from Sect. 4.3.12: $u^{\prime}(0)=V_{0}$. Derive the special formula for $u^{1}$ in this case.

Filename: ic_with_V_0.pdf. 
Open Access This chapter is distributed under the terms of the Creative Commons AttributionNonCommercial 4.0 International License (http://creativecommons.org/licenses/by-nc/4.0/), which permits any noncommercial use, duplication, adaptation, distribution and reproduction in any medium or format, as long as you give appropriate credit to the original author(s) and the source, a link is provided to the Creative Commons license and any changes made are indicated.

The images or other third party material in this chapter are included in the work's Creative Commons license, unless indicated otherwise in the credit line; if such material is not included in the work's Creative Commons license and the respective action is not permitted by statutory regulation, users will need to obtain permission from the license holder to duplicate, adapt or reproduce the material. 


\section{Solving Partial Differential Equations}

The subject of partial differential equations (PDEs) is enormous. At the same time, it is very important, since so many phenomena in nature and technology find their mathematical formulation through such equations. Knowing how to solve at least some PDEs is therefore of great importance to engineers. In an introductory book like this, nowhere near full justice to the subject can be made. However, we still find it valuable to give the reader a glimpse of the topic by presenting a few basic and general methods that we will apply to a very common type of PDE.

We shall focus on one of the most widely encountered partial differential equations: the diffusion equation, which in one dimension looks like

$$
\frac{\partial u}{\partial t}=\beta \frac{\partial^{2} u}{\partial x^{2}}+g .
$$

The multi-dimensional counterpart is often written as

$$
\frac{\partial u}{\partial t}=\beta \nabla^{2} u+g .
$$

We shall restrict the attention here to the one-dimensional case.

The unknown in the diffusion equation is a function $u(x, t)$ of space and time. The physical significance of $u$ depends on what type of process that is described by the diffusion equation. For example, $u$ is the concentration of a substance if the diffusion equation models transport of this substance by diffusion. Diffusion processes are of particular relevance at the microscopic level in biology, e.g., diffusive transport of certain ion types in a cell caused by molecular collisions. There is also diffusion of atoms in a solid, for instance, and diffusion of ink in a glass of water.

One very popular application of the diffusion equation is for heat transport in solid bodies. Then $u$ is the temperature, and the equation predicts how the temperature evolves in space and time within the solid body. For such applications, the equation is known as the heat equation. We remark that the temperature in a fluid is influenced not only by diffusion, but also by the flow of the liquid. If present, the latter effect requires an extra term in the equation (known as an advection or convection term).

The term $g$ is known as the source term and represents generation, or loss, of heat (by some mechanism) within the body. For diffusive transport, $g$ models injection or extraction of the substance. 
We should also mention that the diffusion equation may appear after simplifying more complicated partial differential equations. For example, flow of a viscous fluid between two flat and parallel plates is described by a one-dimensional diffusion equation, where $u$ then is the fluid velocity.

A partial differential equation is solved in some domain $\Omega$ in space and for a time interval $[0, T]$. The solution of the equation is not unique unless we also prescribe initial and boundary conditions. The type and number of such conditions depend on the type of equation. For the diffusion equation, we need one initial condition, $u(x, 0)$, stating what $u$ is when the process starts. In addition, the diffusion equation needs one boundary condition at each point of the boundary $\partial \Omega$ of $\Omega$. This condition can either be that $u$ is known or that we know the normal derivative, $\nabla u \cdot \boldsymbol{n}=\partial u / \partial n(\boldsymbol{n}$ denotes an outward unit normal to $\partial \Omega)$.

Let us look at a specific application and how the diffusion equation with initial and boundary conditions then appears. We consider the evolution of temperature in a one-dimensional medium, more precisely a long rod, where the surface of the rod is covered by an insulating material. The heat can then not escape from the surface, which means that the temperature distribution will only depend on a coordinate along the rod, $x$, and time $t$. At one end of the rod, $x=L$, we also assume that the surface is insulated, but at the other end, $x=0$, we assume that we have some device for controlling the temperature of the medium. Here, a function $s(t)$ tells what the temperature is in time. We therefore have a boundary condition $u(0, t)=s(t)$. At the other insulated end, $x=L$, heat cannot escape, which is expressed by the boundary condition $\partial u(L, t) / \partial x=0$. The surface along the rod is also insulated and hence subject to the same boundary condition (here generalized to $\partial u / \partial n=0$ at the curved surface). However, since we have reduced the problem to one dimension, we do not need this physical boundary condition in our mathematical model. In one dimension, we can set $\Omega=[0, L]$.

To summarize, the partial differential equation with initial and boundary conditions reads

$$
\begin{array}{rlrl}
\frac{\partial u(x, t)}{\partial t} & =\beta \frac{\partial^{2} u(x, t)}{\partial x^{2}}+g(x, t), & & x \in(0, L), t \in(0, T], \\
u(0, t) & =s(t), & & t \in(0, T], \\
\frac{\partial}{\partial x} u(L, t) & =0, & & t \in(0, T], \\
u(x, 0) & =I(x), & x & x[0, L] .
\end{array}
$$

Mathematically, we assume that at $t=0$, the initial condition (5.4) holds and that the partial differential equation (5.1) comes into play for $t>0$. Similarly, at the end points, the boundary conditions (5.2) and (5.3) govern $u$ and the equation therefore is valid for $x \in(0, L)$.

\section{Boundary and initial conditions are needed!}

The initial and boundary conditions are extremely important. Without them, the solution is not unique, and no numerical method will work. Unfortunately, many physical applications have one or more initial or boundary conditions as unknowns. Such situations can be dealt with if we have measurements of $u$, but the mathematical framework is much more complicated. 
What about the source term $g$ in our example with temperature distribution in a rod? $g(x, t)$ models heat generation inside the rod. One could think of chemical reactions at a microscopic level in some materials as a reason to include $g$. However, in most applications with temperature evolution, $g$ is zero and heat generation usually takes place at the boundary (as in our example with $u(0, t)=s(t)$ ).

Before continuing, we may consider an example of how the temperature distribution evolves in the rod. At time $t=0$, we assume that the temperature is $10^{\circ} \mathrm{C}$. Then we suddenly apply a device at $x=0$ that keeps the temperature at $50^{\circ} \mathrm{C}$ at this end. What happens inside the rod? Intuitively, you think that the heat generation at the end will warm up the material in the vicinity of $x=0$, and as time goes by, more and more of the rod will be heated, before the entire rod has a temperature of $50{ }^{\circ} \mathrm{C}$ (recall that no heat escapes from the surface of the rod).

Mathematically, (with the temperature in Kelvin) this example has $I(x)=$ $283 \mathrm{~K}$, except at the end point: $I(0)=323 \mathrm{~K}, s(t)=323 \mathrm{~K}$, and $g=0$. The figure below shows snapshots from four different times in the evolution of the temperature.
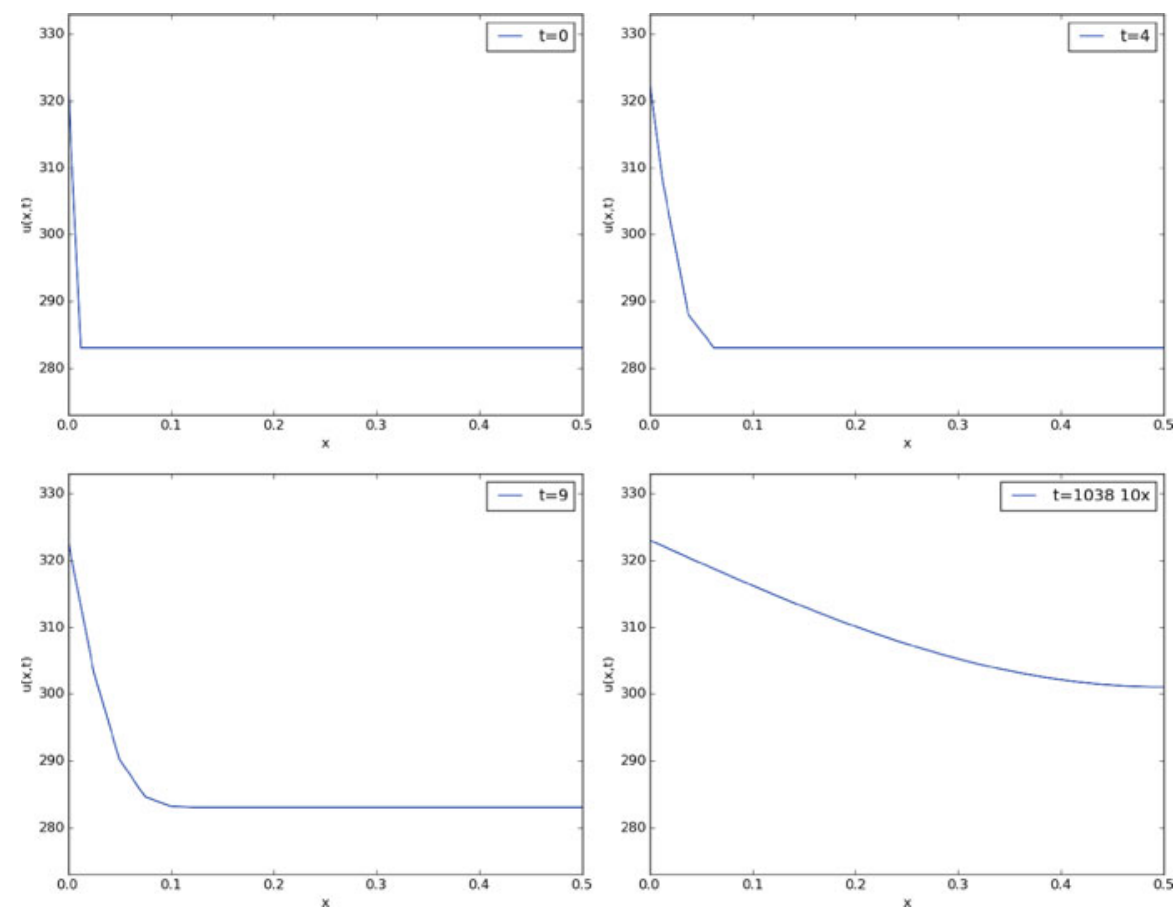

\subsection{Finite Difference Methods}

We shall now construct a numerical method for the diffusion equation. We know how to solve ordinary differential equations, so in a way we are able to deal with the time derivative. Very often in mathematics, a new problem can be solved by reducing it to a series of problems we know how to solve. In the present case, 
it means that we must do something with the spatial derivative $\partial^{2} / \partial x^{2}$ in order to reduce the partial differential equation to ordinary differential equations. One important technique for achieving this, is based on finite difference discretization of spatial derivatives.

\subsubsection{Reduction of a PDE to a System of ODEs}

Introduce a spatial mesh in $\Omega$ with mesh points

$$
x_{0}=0<x_{1}<x_{2}<\cdots<x_{N}=L .
$$

The space between two mesh points $x_{i}$ and $x_{i+1}$, i.e. the interval $\left[x_{i}, x_{i+1}\right]$, is call a cell. We shall here, for simplicity, assume that each cell has the same length $\Delta x=x_{i+1}-x_{i}, i=0, \ldots, N-1$.

The partial differential equation is valid at all spatial points $x \in \Omega$, but we may relax this condition and demand that it is fulfilled at the internal mesh points only, $x_{1}, \ldots, x_{N-1}$ :

$$
\frac{\partial u\left(x_{i}, t\right)}{\partial t}=\beta \frac{\partial^{2} u\left(x_{i}, t\right)}{\partial x^{2}}+g\left(x_{i}, t\right), \quad i=1, \ldots, N-1 .
$$

Now, at any point $x_{i}$ we can approximate the second-order derivative by a finite difference:

$$
\frac{\partial^{2} u\left(x_{i}, t\right)}{\partial x^{2}} \approx \frac{u\left(x_{i+1}, t\right)-2 u\left(x_{i}, t\right)+u\left(x_{i-1}, t\right)}{\Delta x^{2}} .
$$

It is common to introduce a short notation $u_{i}(t)$ for $u\left(x_{i}, t\right)$, i.e., $u$ approximated at some mesh point $x_{i}$ in space. With this new notation we can, after inserting (5.6) in (5.5), write an approximation to the partial differential equation at mesh point $\left(x_{i}, t\right)$ as

$$
\frac{d u_{i}(t)}{d t}=\beta \frac{u_{i+1}(t)-2 u_{i}(t)+u_{i-1}(t)}{\Delta x^{2}}+g_{i}(t), \quad i=1, \ldots, N-1 .
$$

Note that we have adopted the notation $g_{i}(t)$ for $g\left(x_{i}, t\right)$ too.

What is (5.7)? This is nothing but a system of ordinary differential equations in $N-1$ unknowns $u_{1}(t), \ldots, u_{N-1}(t)$ ! In other words, with aid of the finite difference approximation (5.6), we have reduced the single partial differential equation to a system of ODEs, which we know how to solve. In the literature, this strategy is called the method of lines.

We need to look into the initial and boundary conditions as well. The initial condition $u(x, 0)=I(x)$ translates to an initial condition for every unknown function $u_{i}(t): u_{i}(0)=I\left(x_{i}\right), i=0, \ldots, N$. At the boundary $x=0$ we need an ODE in our ODE system, which must come from the boundary condition at this point. The boundary condition reads $u(0, t)=s(t)$. We can derive an ODE from this equation by differentiating both sides: $u_{0}^{\prime}(t)=s^{\prime}(t)$. The ODE system above cannot be used for $u_{0}^{\prime}$ since that equation involves some quantity $u_{-1}^{\prime}$ outside the domain. Instead, we use the equation $u_{0}^{\prime}(t)=s^{\prime}(t)$ derived from the boundary condition. For this 
particular equation we also need to make sure the initial condition is $u_{0}(0)=s(0)$ (otherwise nothing will happen: we get $u=283 \mathrm{~K}$ forever).

We remark that a separate ODE for the (known) boundary condition $u_{0}=s(t)$ is not strictly needed. We can just work with the ODE system for $u_{1}, \ldots, u_{N}$, and in the ODE for $u_{0}$, replace $u_{0}(t)$ by $s(t)$. However, these authors prefer to have an ODE for every point value $u_{i}, i=0, \ldots, N$, which requires formulating the known boundary at $x=0$ as an ODE. The reason for including the boundary values in the ODE system is that the solution of the system is then the complete solution at all mesh points, which is convenient, since special treatment of the boundary values is then avoided.

The condition $\partial u / \partial x=0$ at $x=L$ is a bit more complicated, but we can approximate the spatial derivative by a centered finite difference:

$$
\left.\frac{\partial u}{\partial x}\right|_{i=N} \approx \frac{u_{N+1}-u_{N-1}}{2 \Delta x}=0 .
$$

This approximation involves a fictitious point $x_{N+1}$ outside the domain. A common trick is to use (5.7) for $i=N$ and eliminate $u_{N+1}$ by use of the discrete boundary condition $\left(u_{N+1}=u_{N-1}\right)$ :

$$
\frac{d u_{N}(t)}{d t}=\beta \frac{2 u_{N-1}(t)-2 u_{N}(t)}{\Delta x^{2}}+g_{N}(t) .
$$

That is, we have a special version of (5.7) at the boundary $i=N$.

\section{What about simpler finite differences at the boundary?}

Some reader may think that a smarter trick is to approximate the boundary condition $\partial u / \partial x$ at $x=L$ by a one-sided difference:

$$
\left.\frac{\partial u}{\partial x}\right|_{i=N} \approx \frac{u_{N}-u_{N-1}}{\Delta x}=0 .
$$

This gives a simple equation $u_{N}=u_{N-1}$ for the boundary value, and a corresponding ODE $u_{N}^{\prime}=u_{N-1}^{\prime}$. However, this approximation has an error of order $\Delta x$, while the centered approximation we used above has an error of order $\Delta x^{2}$. The finite difference approximation we used for the second-order derivative in the diffusion equation also has an error of order $\Delta x^{2}$. Thus, if we use the simpler one-sided difference above, it turns out that we reduce the overall accuracy of the method.

We are now in a position to summarize how we can approximate the partial differential equation problem (5.1)-(5.4) by a system of ordinary differential equations:

$$
\begin{aligned}
\frac{d u_{0}}{d t} & =s^{\prime}(t) \\
\frac{d u_{i}}{d t} & =\frac{\beta}{\Delta x^{2}}\left(u_{i+1}(t)-2 u_{i}(t)+u_{i-1}(t)\right)+g_{i}(t), \quad i=1, \ldots, N-1, \\
\frac{d u_{N}}{d t} & =\frac{2 \beta}{\Delta x^{2}}\left(u_{N-1}(t)-u_{N}(t)\right)+g_{N}(t)
\end{aligned}
$$


The initial conditions are

$$
\begin{aligned}
& u_{0}(0)=s(0), \\
& u_{i}(0)=I\left(x_{i}\right), \quad i=1, \ldots, N .
\end{aligned}
$$

We can apply any method for systems of ODEs to solve (5.9)-(5.11).

\subsubsection{Construction of a Test Problem with Known Discrete Solution}

At this point, it is tempting to implement a real physical case and run it. However, partial differential equations constitute a non-trivial topic where mathematical and programming mistakes come easy. A better start is therefore to address a carefully designed test example where we can check that the method works. The most attractive examples for testing implementations are those without approximation errors, because we know exactly what numbers the program should produce. It turns out that solutions $u(x, t)$ that are linear in time and in space can be exactly reproduced by most numerical methods for partial differential equations. A candidate solution might be

$$
u(x, t)=(3 t+2)(x-L) .
$$

Inserting this $u$ in the governing equation gives

$$
3(x-L)=0+g(x, t) \quad \Rightarrow \quad g(x, t)=3(x-L) .
$$

What about the boundary conditions? We realize that $\partial u / \partial x=3 t+2$ for $x=L$, which breaks the assumption of $\partial u / \partial x=0$ at $x=L$ in the formulation of the numerical method above. Moreover, $u(0, t)=-L(3 t+2)$, so we must set $s(t)=$ $-L(3 t+2)$ and $s^{\prime}(t)=-3 L$. Finally, the initial condition dictates $I(x)=2(x-L)$, but recall that we must have $u_{0}=s(0)$, and $u_{i}=I\left(x_{i}\right), i=1, \ldots, N$ : it is important that $u_{0}$ starts out at the right value dictated by $s(t)$ in case $I(0)$ is not equal this value.

First we need to generalize our method to handle $\partial u / \partial x=\gamma \neq 0$ at $x=L$. We then have

$$
\frac{u_{N+1}(t)-u_{N-1}(t)}{2 \Delta x}=\gamma \quad \Rightarrow \quad u_{N+1}=u_{N-1}+2 \gamma \Delta x
$$

which inserted in (5.7) gives

$$
\frac{d u_{N}(t)}{d t}=\beta \frac{2 u_{N-1}(t)+2 \gamma \Delta x-2 u_{N}(t)}{\Delta x^{2}}+g_{N}(t) .
$$

\subsubsection{Implementation: Forward Euler Method}

In particular, we may use the Forward Euler method as implemented in the general function ode_FE from Sect. 4.2.6. The ode_FE function needs a specification of the right-hand side of the ODE system. This is a matter of translating (5.9), (5.10), and (5.14) to Matlab code (in file test_diffusion_pde_exact_linear.m): 


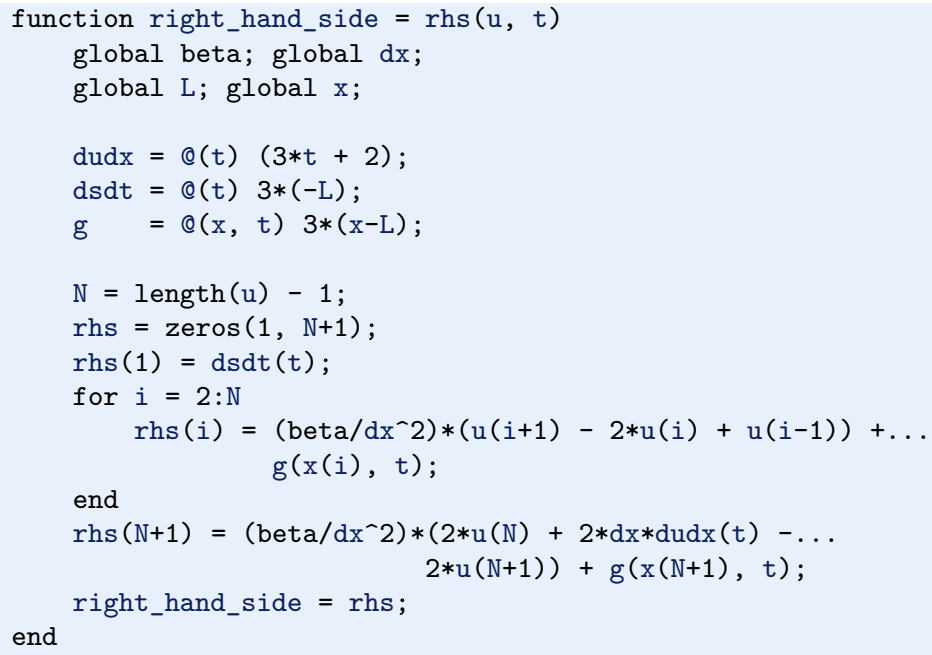

Note that dudx is the function representing the $\gamma$ parameter in (5.14). Also note that the rhs function relies on access to global variables beta, $\mathrm{dx}, \mathrm{L}$, and $\mathrm{x}$, and global functions dsdt, g, and dudx.

We expect the solution to be correct regardless of $N$ and $\Delta t$, so we can choose a small $N, N=4$, and $\Delta t=0.1$. A test function with $N=4$ goes like

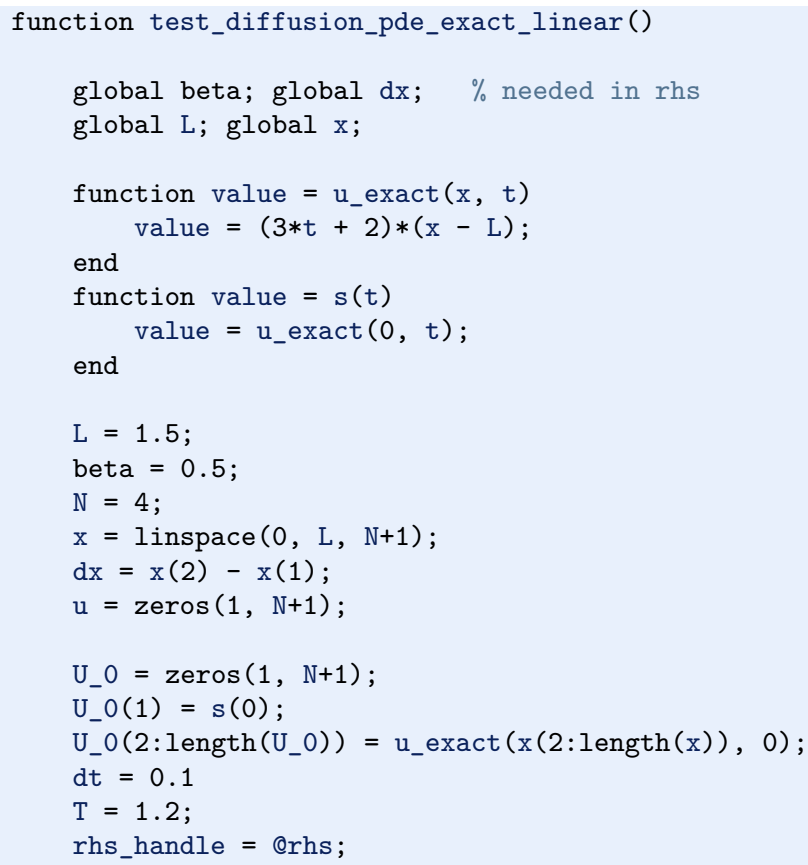




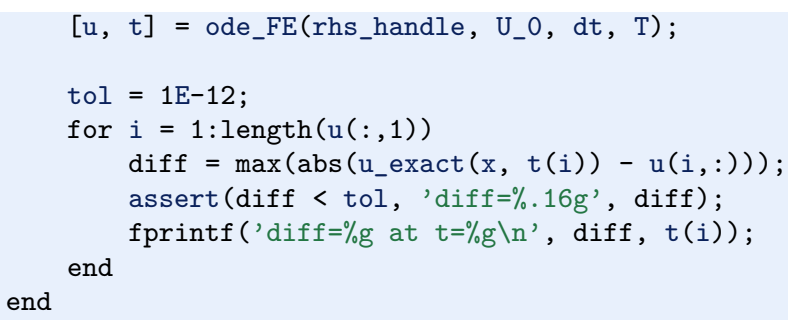

With $N=4$ we reproduce the linear solution exactly. This brings confidence to the implementation, which is just what we need for attacking a real physical problem next.

\subsubsection{Application: Heat Conduction in a Rod}

Let us return to the case with heat conduction in a rod (5.1)-(5.4). Assume that the rod is $50 \mathrm{~cm}$ long and made of aluminum alloy 6082. The $\beta$ parameter equals $\kappa /(\varrho c)$, where $\kappa$ is the heat conduction coefficient, $\varrho$ is the density, and $c$ is the heat capacity. We can find proper values for these physical quantities in the case of aluminum alloy 6082: $\varrho=2.7 \cdot 10^{3} \mathrm{~kg} / \mathrm{m}^{3}, \kappa=200 \frac{\mathrm{W}}{\mathrm{mK}}, c=900 \frac{\mathrm{J}}{\mathrm{Kkg}}$. This results in $\beta=\kappa /(\varrho c)=8.2 \cdot 10^{-5} \mathrm{~m}^{2} / \mathrm{s}$. Preliminary simulations show that we are close to a constant steady state temperature after $1 \mathrm{~h}$, i.e., $T=3600 \mathrm{~s}$.

The functions s, dsdt, f, and dudx must be changed, but the rhs function becomes almost identical to the one from the previous section:

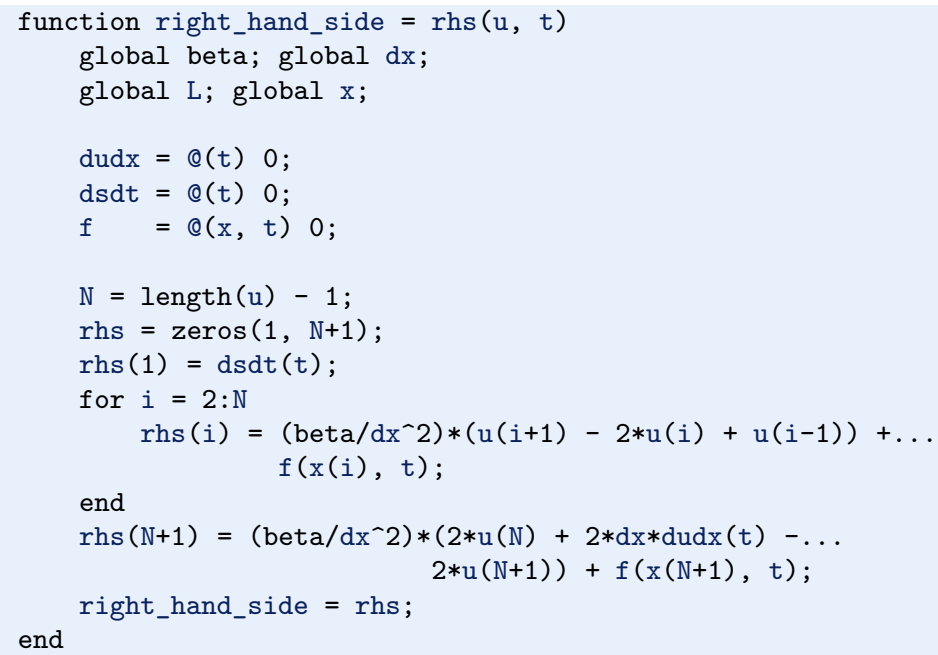

Some new parameter values must also be set, and for the timestep, let us use $\Delta t=0.00034375$. We may also make an animation on the screen to see how $u(x, t)$ develops in time (see file rod_FE.m): 


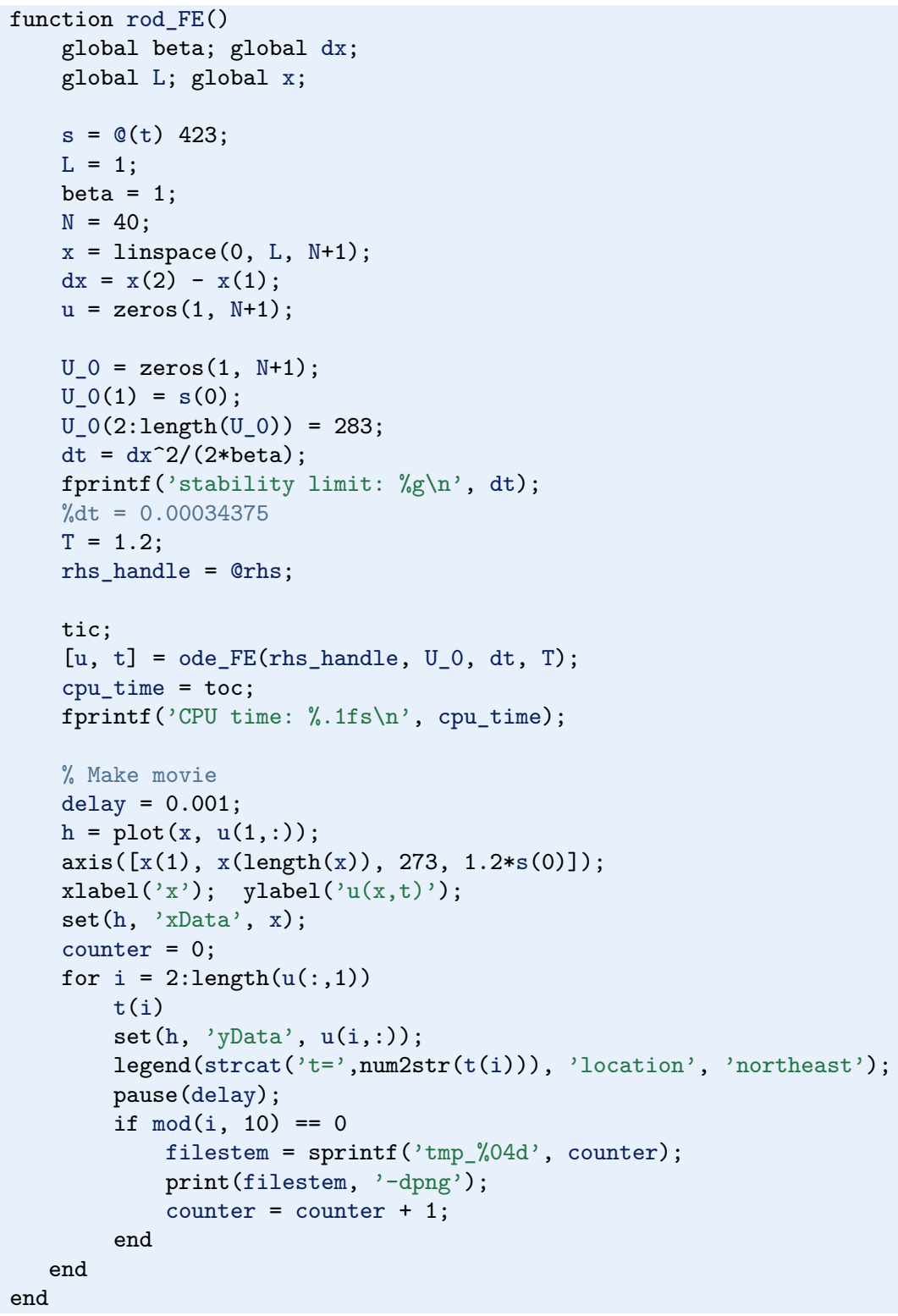

The plotting statements update the $u(x, t)$ curve on the screen. In addition, we save a fraction of the plots to files tmp_0000.png, tmp_0001.png, tmp_0002.png, and so on. These plots can be combined to ordinary video files. A common tool is ffmpeg or its sister avconv.

These programs take the same type of command-line options. To make a Flash video movie.flv, run

Terminal> ffmpeg -i tmp_\%04d.png $-r 4$-vcodec flv movie.flv 

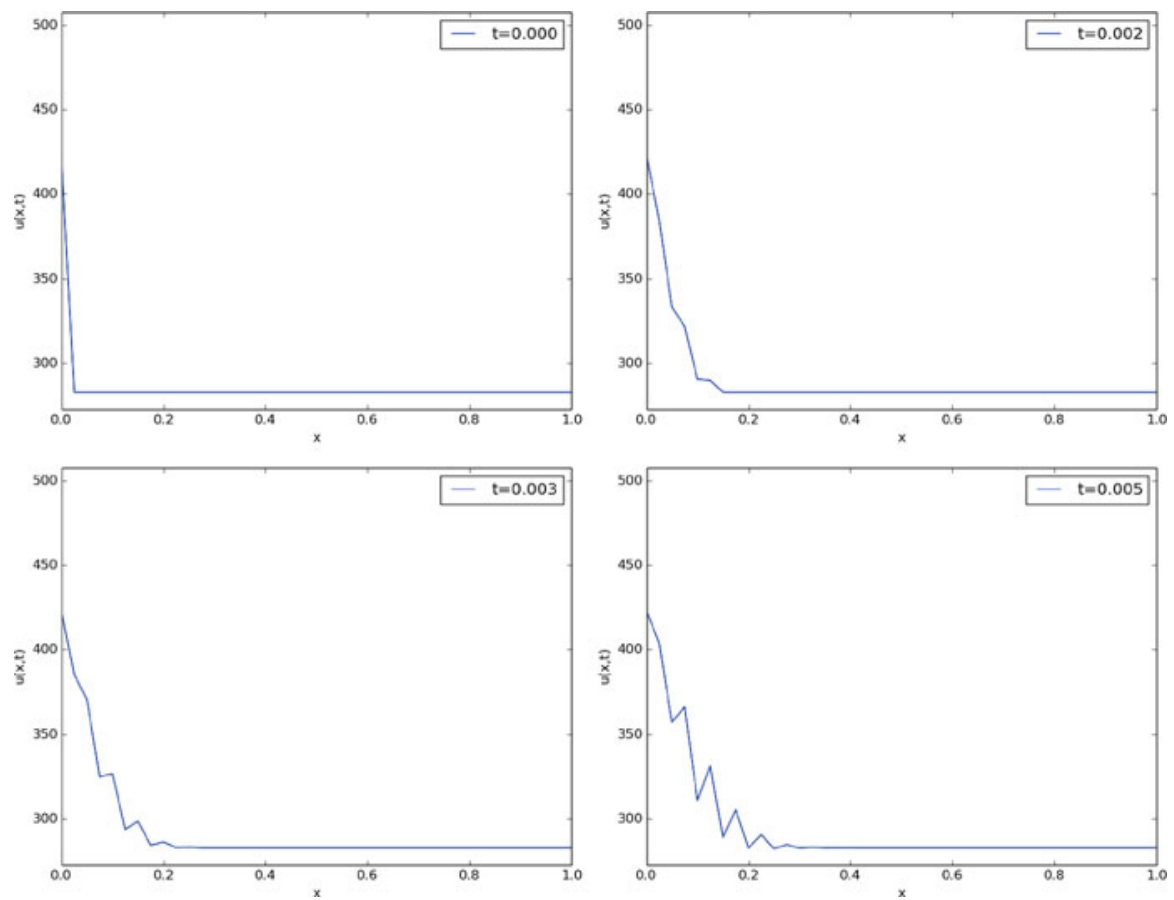

Fig. 5.1 Unstable simulation of the temperature in a rod

The $-i$ option specifies the naming of the plot files in printf syntax, and $-r$ specifies the number of frames per second in the movie. On Mac, run ffmpeg instead of avconv with the same options. Other video formats, such as MP4, WebM, and Ogg can also be produced:

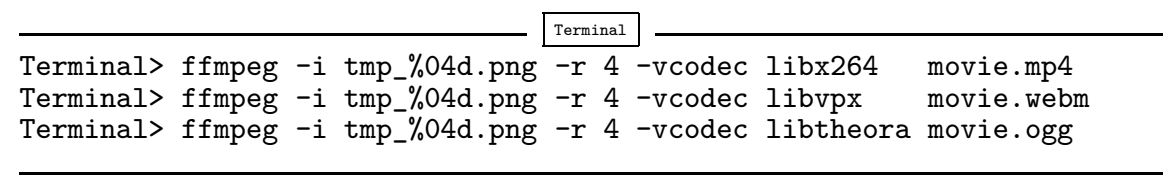

The results of a simulation start out as in Figs. 5.1 and 5.2. We see that the solution definitely looks wrong. The temperature is expected to be smooth, not having such a saw-tooth shape. Also, after some time (Fig. 5.2), the temperature starts to increase much more than expected. We say that this solution is unstable, meaning that it does not display the same characteristics as the true, physical solution. Even though we tested the code carefully in the previous section, it does not seem to work for a physical application! How can that be?

The problem is that $\Delta t$ is too large, making the solution unstable. It turns out that the Forward Euler time integration method puts a restriction on the size of $\Delta t$. For the heat equation and the way we have discretized it, this restriction can be shown to be [10]

$$
\Delta t \leq \frac{\Delta x^{2}}{2 \beta} .
$$



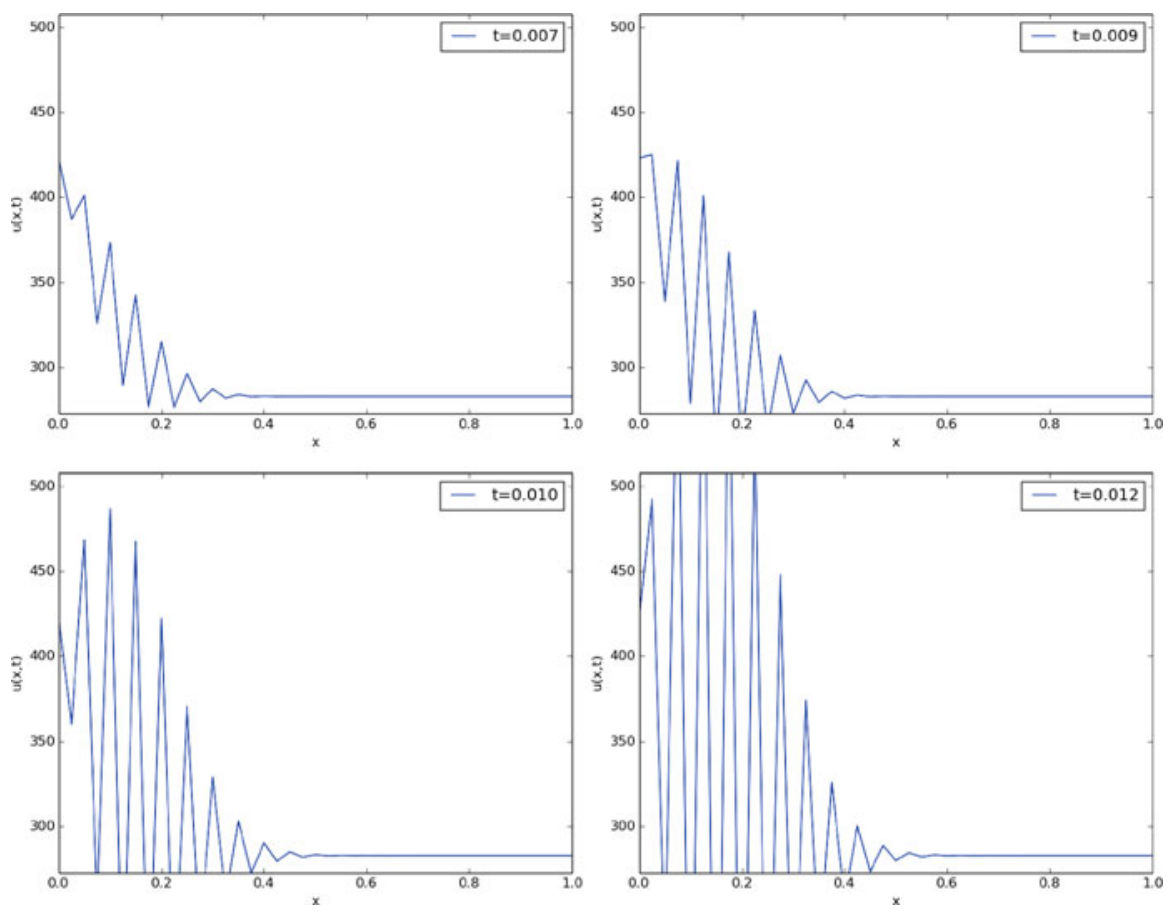

Fig. 5.2 Unstable simulation of the temperature in a rod

This is called a stability criterion. With the chosen parameters, (5.15) tells us that the upper limit is $\Delta t=0.0003125$, which is smaller than our choice above. Rerunning the case with a $\Delta t$ equal to $\Delta x^{2} /(2 \beta)$, indeed shows a smooth evolution of $u(x, t)$. Find the program rod_FE.m and run it to see an animation of the $u(x, t)$ function on the screen.

\section{Scaling and dimensionless quantities}

Our setting of parameters required finding three physical properties of a certain material. The time interval for simulation and the time step depend crucially on the values for $\beta$ and $L$, which can vary significantly from case to case. Often, we are more interested in how the shape of $u(x, t)$ develops, than in the actual $u, x$, and $t$ values for a specific material. We can then simplify the setting of physical parameters by scaling the problem.

Scaling means that we introduce dimensionless independent and dependent variables, here denoted by a bar:

$$
\bar{u}=\frac{u-u^{*}}{u_{c}-u^{*}}, \quad \bar{x}=\frac{x}{x_{c}}, \quad \bar{t}=\frac{t}{t_{c}},
$$

where $u_{c}$ is a characteristic size of the temperature, $u^{*}$ is some reference temperature, while $x_{c}$ and $t_{c}$ are characteristic time and space scales. Here, it is natural to choose $u^{*}$ as the initial condition, and set $u_{c}$ to the stationary (end) temperature. Then $\bar{u} \in[0,1]$, starting at 0 and ending at 1 as $t \rightarrow \infty$. The length $L$ 

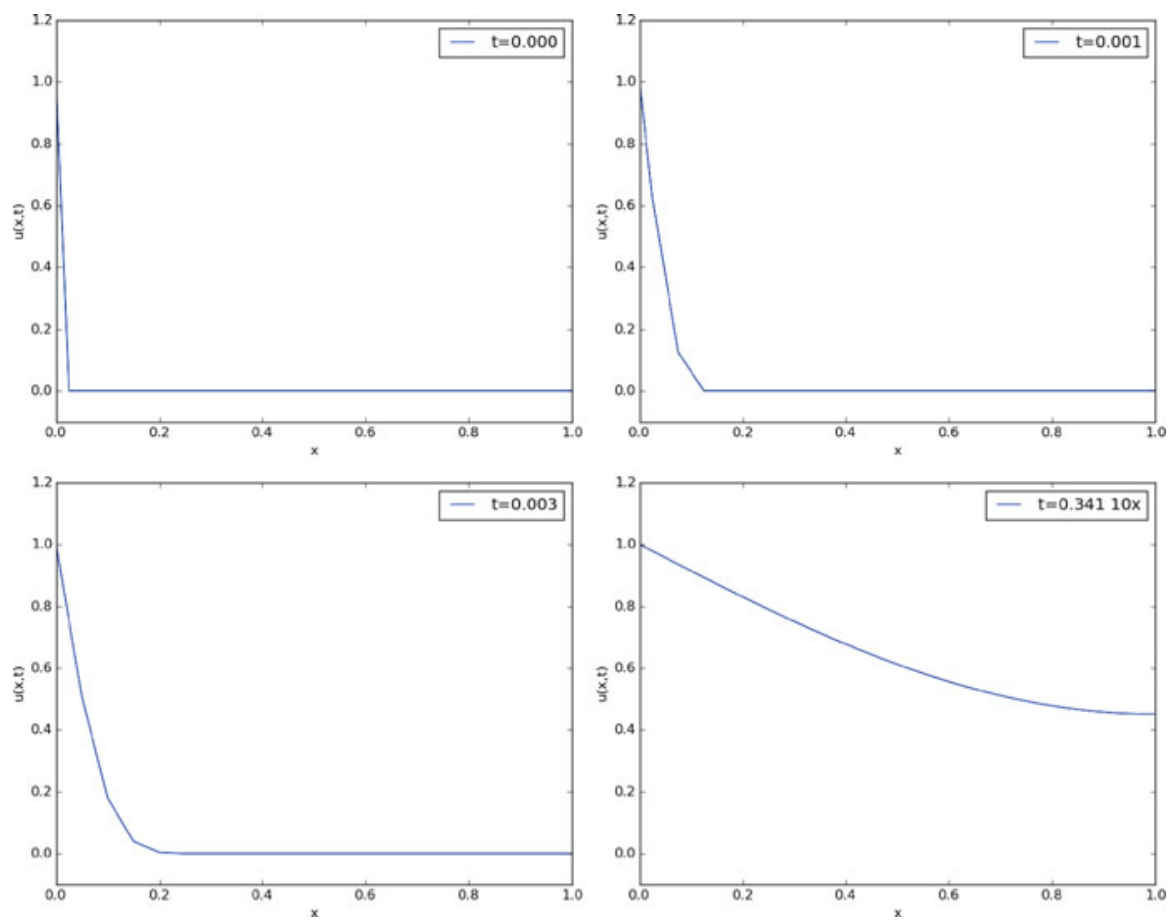

Fig. 5.3 Snapshots of the dimensionless solution of a scaled problem

is $x_{c}$, while choosing $t_{c}$ is more challenging, but one can argue for $t_{c}=L^{2} / \beta$. The resulting equation for $\bar{u}$ reads

$$
\frac{\partial \bar{u}}{\partial \bar{t}}=\frac{\partial^{2} \bar{u}}{\partial \bar{x}^{2}}, \quad \bar{x} \in(0,1) .
$$

Note that in this equation, there are no physical parameters! In other words, we have found a model that is independent of the length of the rod and the material it is made of (!).

We can easily solve this equation with our program by setting $\beta=1, L=1$, $I(x)=0$, and $s(t)=1$. It turns out that the total simulation time (to "infinity") can be taken as 1.2. When we have the solution $\bar{u}(\bar{x}, \bar{t})$, the solution with dimension Kelvin, reflecting the true temperature in our medium, is given by

$$
u(x, t)=u^{*}+\left(u_{c}-u^{*}\right) \bar{u}\left(x / L, t \beta / L^{2}\right) .
$$

Through this formula we can quickly generate the solutions for a rod made of aluminum, wood, or rubber - it is just a matter of plugging in the right $\beta$ value.

Figure 5.3 shows four snapshots of the scaled (dimensionless) solution $\overline{(} \bar{x}, \bar{t})$.

The power of scaling is to reduce the number of physical parameters in a problem, and in the present case, we found one single problem that is independent of the material $(\beta)$ and the geometry $(L)$. 


\subsubsection{Vectorization}

Occasionally in this book, we show how to speed up code by replacing loops over arrays by vectorized expressions. The present problem involves a loop for computing the right-hand side:

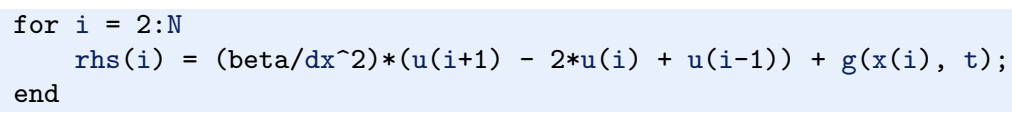

This loop can be replaced by a vectorized expression with the following reasoning. We want to set all the inner points at once: $r$ hs $(2: N)$ (this goes from index 2 up to, and including, N). As the loop index $i$ runs from 2 to $N$, the $u(i+1)$ term will cover all the inner $u$ values displaced one index to the right (compared to $2: \mathrm{N}$ ), i.e., $u(3: N+1)$. Similarly, $u(i-1)$ corresponds to all inner $u$ values displaced one index to the left: $u(1: N-1)$. Finally, $u(i)$ has the same indices as rhs: $u(2: N)$. The vectorized loop can therefore be written in terms of slices:

$$
\operatorname{rhs}(2: N)=\left(\operatorname{beta} / d^{-} 2\right) *(u(3: N+1)-2 * u(2: N)+u(1: N-1))+g(x(2: N), t) ;
$$

This rewrite speeds up the code by about a factor of 10 . A complete code is found in the file rod_FE_vec.m.

\subsubsection{Using Odespy to Solve the System of ODEs}

A nice feature with having a problem defined as a system of ODEs is that we have a rich set of numerical methods available. Matlab/Octave contains general-purpose ODE software such as the ode45 routine that we may apply. However, we shall here step out of the Matlab/Octave world and make use of the Odespy package (see Sect. 4.3.6). Odespy requires the problem to be formulated in Python code. Since Python and Matlab have very similar syntax for the type of programming encountered when using Odespy, it should not be a big step for Matlab/Octave users to utilize Odespy.

Suppose we have defined the right-hand side of our ODE system in a function rhs, the following Python program makes use of Odespy and its adaptive RungeKutta method of order 4-5 (RKFehlberg) to solve the system.

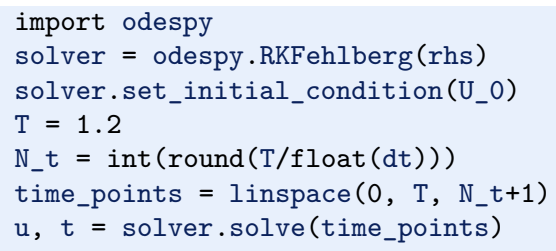



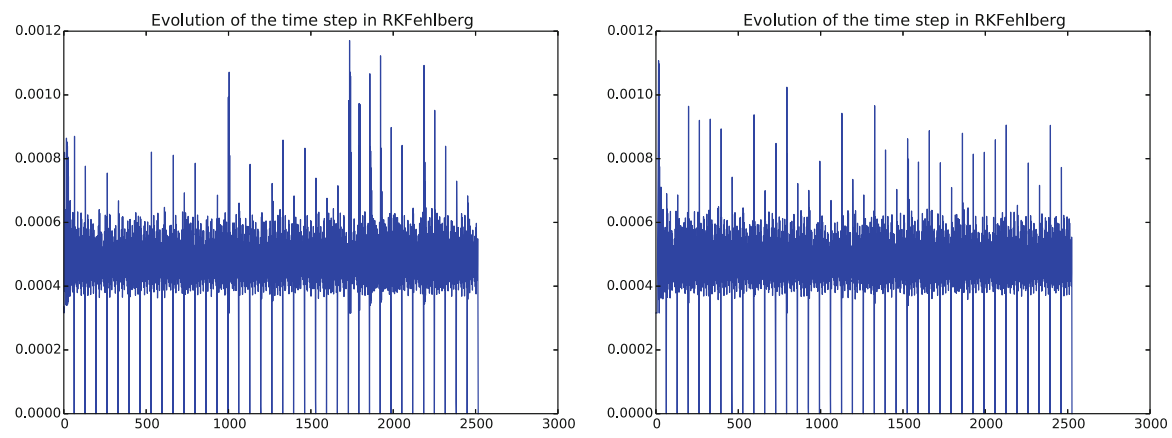

Fig. 5.4 Time steps used by the Runge-Kutta-Fehlberg method: error tolerance $10^{-3}$ (left) and $10^{-6}$ (right)

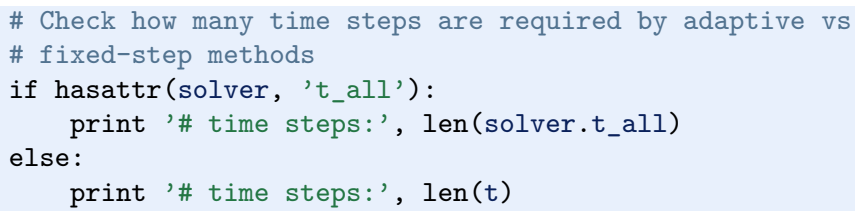

The very nice thing is that we can now easily experiment with many different integration methods. Trying out some simple ones first, like RK2 and RK4, quickly reveals that the time step limitation of the Forward Euler scheme also applies to these more sophisticated Runge-Kutta methods, but their accuracy is better. However, the Odespy package offers also adaptive methods. We can then specify a much larger time step in time_points, and the solver will figure out the appropriate step. Above we indicated how to use the adaptive Runge-Kutta-Fehlberg 4-5 solver. While the $\Delta t$ corresponding to the Forward Euler method requires over 8000 steps for a simulation, we started the RKFehlberg method with 100 times this time step and in the end it required just slightly more than 2500 steps, using the default tolerance parameters. Lowering the tolerance did not save any significant amount of computational work. Figure 5.4 shows a comparison of the length of all the time steps for two values of the tolerance. We see that the influence of the tolerance is minor in this computational example, so it seems that the blow-up due to instability is what governs the time step size. The nice feature of this adaptive method is that we can just specify when we want the solution to be computed, and the method figures out on its own what time step that has to be used because of stability restrictions.

We have seen how easy it is to apply sophisticated methods for ODEs to this PDE example. We shall take the use of Odespy one step further in the next section.

\subsubsection{Implicit Methods}

A major problem with the stability criterion (5.15) is that the time step becomes very small if $\Delta x$ is small. For example, halving $\Delta x$ requires four times as many time steps and eight times the work. Now, with $N=40$, which is a reasonable resolution for the test problem above, the computations are very fast. What takes 
time, is the visualization on the screen, but for that purpose one can visualize only a subset of the time steps. However, there are occasions when you need to take larger time steps with the diffusion equation, especially if interest is in the longterm behavior as $t \rightarrow \infty$. You must then turn to implicit methods for ODEs. These methods require the solutions of linear systems, if the underlying PDE is linear, and systems of nonlinear algebraic equations if the underlying PDE is non-linear.

The simplest implicit method is the Backward Euler scheme, which puts no restrictions on $\Delta t$ for stability, but obviously, a large $\Delta t$ leads to inaccurate results. The Backward Euler scheme for a scalar ODE $u^{\prime}=f(u, t)$ reads

$$
\frac{u^{n+1}-u^{n}}{\Delta t}=f\left(u^{n+1}, t_{n+1}\right) \text {. }
$$

This equation is to be solved for $u^{n+1}$. If $f$ is linear in $u$, it is a linear equation, but if $f$ is nonlinear in $u$, one needs approximate methods for nonlinear equations (Chap. 6).

In our case, we have a system of linear ODEs (5.9)-(5.11). The Backward Euler scheme applied to each equation leads to

$$
\begin{aligned}
& \frac{u_{0}^{n+1}-u_{0}^{n}}{\Delta t}=s^{\prime}\left(t_{n+1}\right) \\
& \frac{u_{i}^{n+1}-u_{i}^{n}}{\Delta t}=\frac{\beta}{\Delta x^{2}}\left(u_{i+1}^{n+1}-2 u_{i}^{n+1}+u_{i-1}^{n+1}\right)+g_{i}\left(t_{n+1}\right), \\
& \quad i=1, \ldots, N-1, \\
& \frac{u_{N}^{n+1}-u_{N}^{n}}{\Delta t}=\frac{2 \beta}{\Delta x^{2}}\left(u_{N-1}^{n+1}-u_{N}^{n+1}\right)+g_{i}\left(t_{n+1}\right)
\end{aligned}
$$

This is a system of linear equations in the unknowns $u_{i}^{n+1}, i=0, \ldots, N$, which is easy to realize by writing out the equations for the case $N=3$, collecting all the unknown terms on the left-hand side and all the known terms on the right-hand side:

$$
\begin{aligned}
u_{0}^{n+1} & =u_{0}^{n}+\Delta t s^{\prime}\left(t_{n+1}\right), \\
u_{1}^{n+1}-\Delta t \frac{\beta}{\Delta x^{2}}\left(u_{2}^{n+1}-2 u_{1}^{n+1}+u_{0}^{n+1}\right) & =u_{1}^{n}+\Delta t g_{1}\left(t_{n+1}\right), \\
u_{2}^{n+1}-\Delta t \frac{2 \beta}{\Delta x^{2}}\left(u_{1}^{n+1}-u_{2}^{n+1}\right) & =u_{2}^{n}+\Delta t g_{2}\left(t_{n+1}\right) .
\end{aligned}
$$

A system of linear equations like this, is usually written on matrix form $A u=b$, where $A$ is a coefficient matrix, $u=\left(u_{0}^{n+1}, \ldots, n_{N}^{n+1}\right)$ is the vector of unknowns, and $b$ is a vector of known values. The coefficient matrix for the case (5.19)-(5.21) becomes

$$
A=\left(\begin{array}{ccc}
1 & 0 & 0 \\
-\Delta t \frac{\beta}{\Delta x^{2}} & 1+2 \Delta t \frac{\beta}{\Delta x^{2}} & -\Delta t \frac{\beta}{\Delta x^{2}} \\
0 & -\Delta t \frac{2 \beta}{\Delta x^{2}} & 1+\Delta t \frac{2 \beta}{\Delta x^{2}}
\end{array}\right)
$$


In the general case (5.16)-(5.18), the coefficient matrix is an $(N+1) \times(N+1)$ matrix with zero entries, except for

$$
\begin{aligned}
A_{1,1} & =1 \\
A_{i, i-1} & =-\Delta t \frac{\beta}{\Delta x^{2}}, \quad i=2, \ldots, N-1 \\
A_{i, i+1} & =-\Delta t \frac{\beta}{\Delta x^{2}}, \quad i=2, \ldots, N-1 \\
A_{i, i} & =1+2 \Delta t \frac{\beta}{\Delta x^{2}}, \quad i=2, \ldots, N-1 \\
A_{N, N-1} & =-\Delta t \frac{2 \beta}{\Delta x^{2}} \\
A_{N, N} & =1+\Delta t \frac{2 \beta}{\Delta x^{2}}
\end{aligned}
$$

If we want to apply general methods for systems of ODEs on the form $u^{\prime}=$ $f(u, t)$, we can assume a linear $f(u, t)=K u$. The coefficient matrix $K$ is found from the right-hand side of (5.16)-(5.18) to be

$$
\begin{aligned}
K_{1,1} & =0 \\
K_{i, i-1} & =\frac{\beta}{\Delta x^{2}}, \quad i=2, \ldots, N-1 \\
K_{i, i+1} & =\frac{\beta}{\Delta x^{2}}, \quad i=2, \ldots, N-1 \\
K_{i, i} & =-\frac{2 \beta}{\Delta x^{2}}, \quad i=2, \ldots, N-1 \\
K_{N, N-1} & =\frac{2 \beta}{\Delta x^{2}} \\
K_{N, N} & =-\frac{2 \beta}{\Delta x^{2}}
\end{aligned}
$$

We see that $A=I-\Delta t K$.

To implement the Backward Euler scheme, we can either fill a matrix and call a linear solver, or we can apply Odespy. We follow the latter strategy. Implicit methods in Odespy need the $K$ matrix above, given as an argument jac (Jacobian of $f$ ) in the call to odespy.BackwardEuler. Here is the Python code for the right-hand side of the ODE system (rhs) and the $K$ matrix (K) as well as statements for initializing and running the Odespy solver BackwardEuler (in the file rod_BE.py):

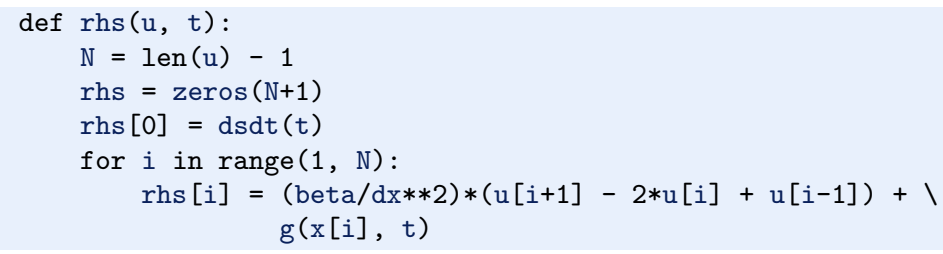




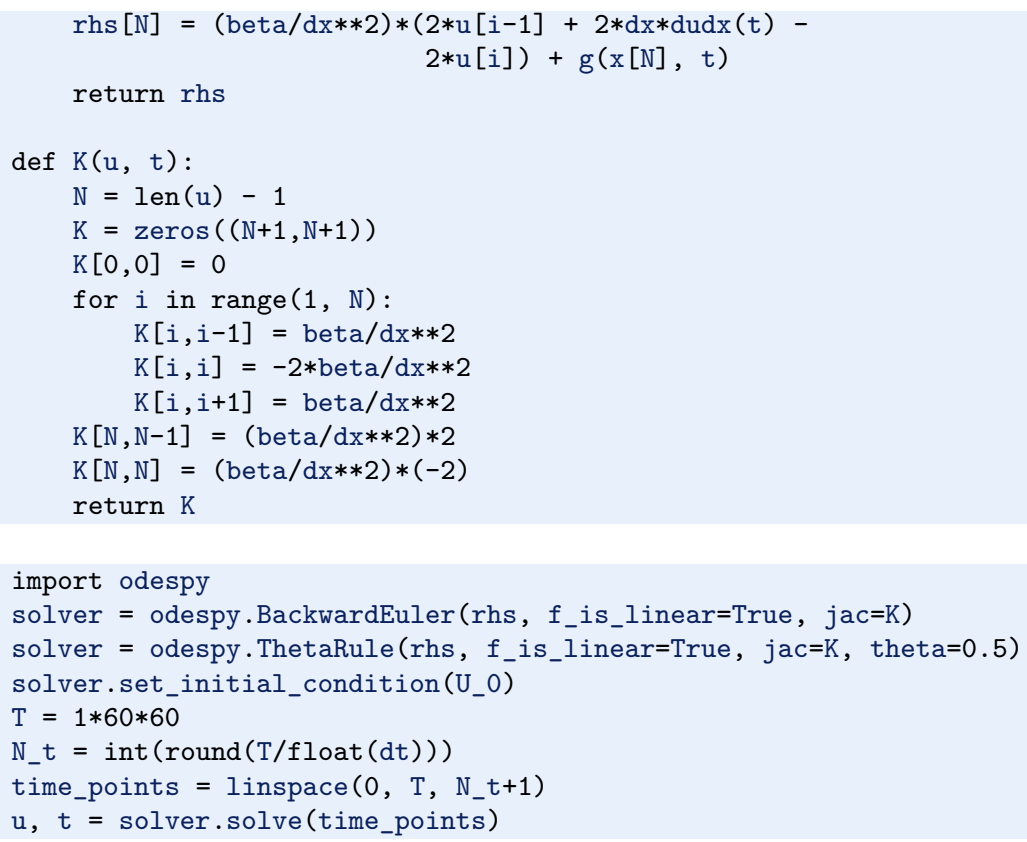

The file rod_BE.py has all the details and shows a movie of the solution. We can run it with any $\Delta t$ we want, its size just impacts the accuracy of the first steps.

\section{Odespy solvers apply dense matrices!}

Looking at the entries of the $K$ matrix, we realize that there are at maximum three entries different from zero in each row. Therefore, most of the entries are zeroes. The Odespy solvers expect dense square matrices as input, here with $(N+1) \times(N+1)$ elements. When solving the linear systems, a lot of storage and work are spent on the zero entries in the matrix. It would be much more efficient to store the matrix as a tridiagonal matrix and apply a specialized Gaussian elimination solver for tridiagonal systems. Actually, this reduces the work from the order $N^{3}$ to the order $N$.

In one-dimensional diffusion problems, the savings of using a tridiagonal matrix are modest in practice, since the matrices are very small anyway. In two- and three-dimensional PDE problems, however, one cannot afford dense square matrices. Rather, one must resort to more efficient storage formats and algorithms tailored to such formats, but this is beyond the scope of the present text.

\subsection{Exercises}

\section{Exercise 5.1: Simulate a diffusion equation by hand}

Consider the problem given by (5.9), (5.10) and (5.14). Set $N=2$ and compute $u_{i}^{0}, u_{i}^{1}$ and $u_{i}^{2}$ by hand for $i=0,1,2$. Use these values to construct a test function for checking that the implementation is correct. Copy useful functions from test_diffusion_pde_exact_linear.m and make a new test function test_diffusion_hand_calculation.

Filename: test_rod_hand_calculations.m. 


\section{Exercise 5.2: Compute temperature variations in the ground}

The surface temperature at the ground shows daily and seasonal oscillations. When the temperature rises at the surface, heat is propagated into the ground, and the coefficient $\beta$ in the diffusion equation determines how fast this propagation is. It takes some time before the temperature rises down in the ground. At the surface, the temperature has then fallen. We are interested in how the temperature varies down in the ground because of temperature oscillations on the surface.

Assuming homogeneous horizontal properties of the ground, at least locally, and no variations of the temperature at the surface at a fixed point of time, we can neglect the horizontal variations of the temperature. Then a one-dimensional diffusion equation governs the heat propagation along a vertical axis called $x$. The surface corresponds to $x=0$ and the $x$ axis point downwards into the ground. There is no source term in the equation (actually, if rocks in the ground are radioactive, they emit heat and that can be modeled by a source term, but this effect is neglected here).

At some depth $x=L$ we assume that the heat changes in $x$ vanish, so $\partial u / \partial x=$ 0 is an appropriate boundary condition at $x=L$. We assume a simple sinusoidal temperature variation at the surface:

$$
u(0, t)=T_{0}+T_{a} \sin \left(\frac{2 \pi}{P} t\right)
$$

where $P$ is the period, taken here as 24 hours $(24 \cdot 60 \cdot 60 \mathrm{~s})$. The $\beta$ coefficient may be set to $10^{-6} \mathrm{~m}^{2} / \mathrm{s}$. Time is then measured in seconds. Set appropriate values for $T_{0}$ and $T_{a}$.

a) Show that the present problem has an analytical solution of the form

$$
u(x, t)=A+B e^{-r x} \sin (\omega t-r x),
$$

for appropriate values of $A, B, r$, and $\omega$.

b) Solve this heat propagation problem numerically for some days and animate the temperature. You may use the Forward Euler method in time. Plot both the numerical and analytical solution. As initial condition for the numerical solution, use the exact solution during program development, and when the curves coincide in the animation for all times, your implementation works, and you can then switch to a constant initial condition: $u(x, 0)=T_{0}$. For this latter initial condition, how many periods of oscillations are necessary before there is a good (visual) match between the numerical and exact solution (despite differences at $t=0)$ ?

Filename: ground_temp.m.

\section{Exercise 5.3: Compare implicit methods}

An equally stable, but more accurate method than the Backward Euler scheme, is the so-called 2-step backward scheme, which for an ODE $u^{\prime}=f(u, t)$ can be expressed by

$$
\frac{3 u^{n+1}-4 u^{n}+u^{n-1}}{2 \Delta t}=f\left(u^{n+1}, t_{n+1}\right) .
$$


The Odespy package offers this method as odespy. Backward2Step. The purpose of this exercise is to compare three methods and animate the three solutions:

1. The Backward Euler method with $\Delta t=0.001$

2. The backward 2-step method with $\Delta t=0.001$

3. The backward 2-step method with $\Delta t=0.01$

Choose the model problem from Sect. 5.1.4.

Filename: rod_BE_vs_B2Step.m.

\section{Exercise 5.4: Explore adaptive and implicit methods}

We consider the same problem as in Exercise 5.2. Now we want to explore the use of adaptive and implicit methods from Odespy to see if they are more efficient than the Forward Euler method. Assume that you want the accuracy provided by the Forward Euler method with its maximum $\Delta t$ value. Since there exists an analytical solution, you can compute an error measure that summarizes the error in space and time over the whole simulation:

$$
E=\sqrt{\Delta x \Delta t \sum_{i} \sum_{n}\left(U_{i}^{n}-u_{i}^{n}\right)^{2}} .
$$

Here, $U_{i}^{n}$ is the exact solution. Use the Odespy package to run the following implicit and adaptive solvers:

1. BackwardEuler

2. Backward2Step

3. RKFehlberg

Experiment to see if you can use larger time steps than what is required by the Forward Euler method and get solutions with the same order of accuracy.

Hint To avoid oscillations in the solutions when using the RKFehlberg method, the rtol and atol parameters to RKFFehlberg must be set no larger than 0.001 and 0.0001 , respectively. You can print out solver_RKF.t_all to see all the time steps used by the RKFehlberg solver (if solver is the RKFehlberg object). You can then compare the number of time steps with what is required by the other methods.

Filename: ground_temp_adaptive.m.

\section{Exercise 5.5: Investigate the $\theta$ rule}

a) The Crank-Nicolson method for ODEs is very popular when combined with diffusion equations. For a linear ODE $u^{\prime}=a u$ it reads

$$
\frac{u^{n+1}-u^{n}}{\Delta t}=\frac{1}{2}\left(a u^{n}+a u^{n+1}\right) \text {. }
$$

Apply the Crank-Nicolson method in time to the ODE system for a onedimensional diffusion equation. Identify the linear system to be solved. 
b) The Backward Euler, Forward Euler, and Crank-Nicolson methods can be given a unified implementation. For a linear ODE $u^{\prime}=a u$ this formulation is known as the $\theta$ rule:

$$
\frac{u^{n+1}-u^{n}}{\Delta t}=(1-\theta) a u^{n}+\theta a u^{n+1} .
$$

For $\theta=0$ we recover the Forward Euler method, $\theta=1$ gives the Backward Euler scheme, and $\theta=1 / 2$ corresponds to the Crank-Nicolson method. The approximation error in the $\theta$ rule is proportional to $\Delta t$, except for $\theta=1 / 2$ where it is proportional to $\Delta t^{2}$. For $\theta \geq 1 / 2$ the method is stable for all $\Delta t$.

Apply the $\theta$ rule to the ODE system for a one-dimensional diffusion equation. Identify the linear system to be solved.

c) Implement the $\theta$ rule with aid of the Odespy package. The relevant object name is ThetaRule:

solver = odespy.ThetaRule(rhs, f_is_linear=True, jac=K, theta=0.5)

d) Consider the physical application from Sect. 5.1.4. Run this case with the $\theta$ rule and $\theta=1 / 2$ for the following values of $\Delta t: 0.001,0.01,0.05$. Report what you see.

Filename: rod_ThetaRule.m.

Remarks Despite the fact that the Crank-Nicolson method, or the $\theta$ rule with $\theta=$ $1 / 2$, is theoretically more accurate than the Backward Euler and Forward Euler schemes, it may exhibit non-physical oscillations as in the present example if the solution is very steep. The oscillations are damped in time, and decreases with decreasing $\Delta t$. To avoid oscillations one must have $\Delta t$ at maximum twice the stability limit of the Forward Euler method. This is one reason why the Backward Euler method (or a 2-step backward scheme, see Exercise 5.3) are popular for diffusion equations with abrupt initial conditions.

Exercise 5.6: Compute the diffusion of a Gaussian peak

Solve the following diffusion problem:

$$
\begin{array}{rlrl}
\frac{\partial u}{\partial t} & =\beta \frac{\partial^{2} u}{\partial x^{2}}, & x \in(-1,1), t \in(0, T] \\
u(x, 0) & =\frac{1}{\sqrt{2 \pi} \sigma} \exp \left(-\frac{x^{2}}{2 \sigma^{2}}\right), & x \in[-1,1], \\
\frac{\partial}{\partial x} u(-1, t) & =0 & t \in(0, T], \\
\frac{\partial}{\partial x} u(1, t) & =0 & t \in(0, T] .
\end{array}
$$

The initial condition is the famous and widely used Gaussian function with standard deviation (or "width") $\sigma$, which is here taken to be small, $\sigma=0.01$, such that the initial condition is a peak. This peak will then diffuse and become lower and wider. Compute $u(x, t)$ until $u$ becomes approximately constant over the domain.

Filename: gaussian_diffusion.m. 
Remarks Running the simulation with $\sigma=0.2$ results in a constant solution $u \approx 1$ as $t \rightarrow \infty$, while one might expect from "physics of diffusion" that the solution should approach zero. The reason is that we apply Neumann conditions as boundary conditions. One can then easily show that the area under the $u$ curve remains constant. Integrating the PDE gives

$$
\int_{-1}^{1} \frac{\partial u}{\partial t} d x=\beta \int_{-1}^{1} \frac{\partial d^{2} u}{\partial x^{2}} d x
$$

Using the Gauss divergence theorem on the integral on the right-hand and moving the time-derivative outside the integral on the left-hand side results in

$$
\frac{\partial}{\partial t} \int_{-1}^{1} u(x, t) d x=\beta\left[\frac{\partial d u}{\partial x}\right]_{-1}^{1}=0 .
$$

(Recall that $\partial u / \partial x=0$ at the end points.) The result means that $\int_{-1}^{1} u d x$ remains constant during the simulation. Giving the PDE an interpretation in terms of heat conduction can easily explain the result: with Neumann conditions no heat can escape from the domain so the initial heat will just be evenly distributed, but not leak out, so the temperature cannot go to zero (or the scaled and translated temperature $u$, to be precise). The area under the initial condition is 1 , so with a sufficiently fine mesh, $u \rightarrow 1$, regardless of $\sigma$.

\section{Exercise 5.7: Vectorize a function for computing the area of a polygon}

Vectorize the implementation of the function for computing the area of a polygon in Exercise 2.5. Make a test function that compares the scalar implementation in Exercise 2.5 and the new vectorized implementation for the test cases used in Exercise 2.5.

Hint Notice that the formula $x_{1} y_{2}+x_{2} y_{3}+\cdots+x_{n-1} y_{n}=\sum_{i=0}^{n-1} x_{i} y_{i+1}$ is the dot product of two vectors, $\mathrm{x}(1$ : end -1$)$ and $\mathrm{y}(2$, end $)$, which can be computed as $\operatorname{dot}(\mathrm{x}(1:$ end -1$), \mathrm{y}(2$, end $))$, or more explicitly as $\operatorname{sum}(\mathrm{x}(1:$ end -1$) \cdot * \mathrm{y}(1$ : end)).

Filename: polyarea_vec.m.

\section{Exercise 5.8: Explore symmetry}

One can observe (and also mathematically prove) that the solution $u(x, t)$ of the problem in Exercise 5.6 is symmetric around $x=0: u(-x, t)=u(x, t)$. In such a case, we can split the domain in two and compute $u$ in only one half, $[-1,0]$ or $[0,1]$. At the symmetry line $x=0$ we have the symmetry boundary condition $\partial u / \partial x=0$. Reformulate the problem in Exercise 5.6 such that we compute only for $x \in[0,1]$. Display the solution and observe that it equals the right part of the solution in Exercise 5.6.

Filename: symmetric_gaussian_diffusion.m. 
Remarks In 2D and 3D problems, where the CPU time to compute a solution of PDE can be hours and days, it is very important to utilize symmetry as we do above to reduce the size of the problem.

Also note the remarks in Exercise 5.6 about the constant area under the $u(x, t)$ curve: here, the area is 0.5 and $u \rightarrow 0.5$ as $t \rightarrow 0.5$ (if the mesh is sufficiently fine - one will get convergence to smaller values for small $\sigma$ if the mesh is not fine enough to properly resolve a thin-shaped initial condition).

\section{Exercise 5.9: Compute solutions as $t \rightarrow \infty$}

Many diffusion problems reach a stationary time-independent solution as $t \rightarrow \infty$. The model problem from Sect. 5.1.4 is one example where $u(x, t)=s(t)=$ const for $t \rightarrow \infty$. When $u$ does not depend on time, the diffusion equation reduces to

$$
-\beta u^{\prime \prime}(x)=f(x)
$$

in one dimension, and

$$
-\beta \nabla^{2} u=f(x)
$$

in $2 \mathrm{D}$ and 3D. This is the famous Poisson equation, or if $f=0$, it is known as the Laplace equation. In this limit $t \rightarrow \infty$, there is no need for an initial condition, but the boundary conditions are the same as for the diffusion equation.

We now consider a one-dimensional problem

$$
-u^{\prime \prime}(x)=0, x \in(0, L), \quad u(0)=C, u^{\prime}(L)=0,
$$

which is known as a two-point boundary value problem. This is nothing but the stationary limit of the diffusion problem in Sect. 5.1.4. How can we solve such a stationary problem (5.38)? The simplest strategy, when we already have a solver for the corresponding time-dependent problem, is to use that solver and simulate until $t \rightarrow \infty$, which in practice means that $u(x, t)$ no longer changes in time (within some tolerance).

A nice feature of implicit methods like the Backward Euler scheme is that one can take one very long time step to "infinity" and produce the solution of (5.38).

a) Let (5.38) be valid at mesh points $x_{i}$ in space, discretize $u^{\prime \prime}$ by a finite difference, and set up a system of equations for the point values $u_{i}, i=0, \ldots, N$, where $u_{i}$ is the approximation at mesh point $x_{i}$.

b) Show that if $\Delta t \rightarrow \infty$ in (5.16)-(5.18), it leads to the same equations as in a).

c) Demonstrate, by running a program, that you can take one large time step with the Backward Euler scheme and compute the solution of (5.38). The solution is very boring since it is constant: $u(x)=C$.

Filename: rod_stationary.m.

Remarks If the interest is in the stationary limit of a diffusion equation, one can either solve the associated Laplace or Poisson equation directly, or use a Backward Euler scheme for the time-dependent diffusion equation with a very long time step. Using a Forward Euler scheme with small time steps is typically inappropriate in 
such situations because the solution changes more and more slowly, but the time step must still be kept small, and it takes "forever" to approach the stationary state. This is yet another example why one needs implicit methods like the Backward Euler scheme.

\section{Exercise 5.10: Solve a two-point boundary value problem}

Solve the following two-point boundary-value problem

$$
u^{\prime \prime}(x)=2, x \in(0,1), \quad u(0)=0, u(1)=1 .
$$

Hint Do Exercise 5.9. Modify the boundary condition in the code so it incorporates a known value for $u(1)$.

Filename: 2ptBVP.m.

Open Access This chapter is distributed under the terms of the Creative Commons AttributionNonCommercial 4.0 International License (http://creativecommons.org/licenses/by-nc/4.0/), which permits any noncommercial use, duplication, adaptation, distribution and reproduction in any medium or format, as long as you give appropriate credit to the original author(s) and the source, a link is provided to the Creative Commons license and any changes made are indicated.

The images or other third party material in this chapter are included in the work's Creative Commons license, unless indicated otherwise in the credit line; if such material is not included in the work's Creative Commons license and the respective action is not permitted by statutory regulation, users will need to obtain permission from the license holder to duplicate, adapt or reproduce the material. 


\section{Solving Nonlinear Algebraic Equations}
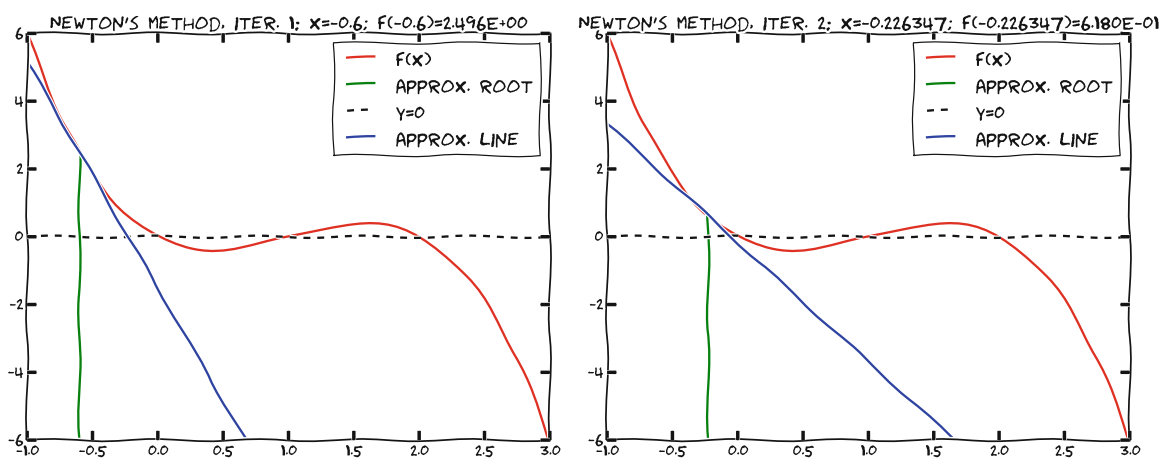

As a reader of this book you are probably well into mathematics and often "accused" of being particularly good at "solving equations" (a typical comment at family dinners!). However, is it really true that you, with pen and paper, can solve many types of equations? Restricting our attention to algebraic equations in one unknown $x$, you can certainly do linear equations: $a x+b=0$, and quadratic ones: $a x^{2}+b x+c=0$. You may also know that there are formulas for the roots of cubic and quartic equations too. Maybe you can do the special trigonometric equation $\sin x+\cos x=1$ as well, but there it (probably) stops. Equations that are not reducible to one of the mentioned cannot be solved by general analytical techniques, which means that most algebraic equations arising in applications cannot be treated with pen and paper!

If we exchange the traditional idea of finding exact solutions to equations with the idea of rather finding approximate solutions, a whole new world of possibilities opens up. With such an approach, we can in principle solve any algebraic equation. 
Let us start by introducing a common generic form for any algebraic equation:

$$
f(x)=0 .
$$

Here, $f(x)$ is some prescribed formula involving $x$. For example, the equation

$$
e^{-x} \sin x=\cos x
$$

has

$$
f(x)=e^{-x} \sin x-\cos x .
$$

Just move all terms to the left-hand side and then the formula to the left of the equality sign is $f(x)$.

So, when do we really need to solve algebraic equations beyond the simplest types we can treat with pen and paper? There are two major application areas. One is when using implicit numerical methods for ordinary differential equations. These give rise to one or a system of algebraic equations. The other major application type is optimization, i.e., finding the maxima or minima of a function. These maxima and minima are normally found by solving the algebraic equation $F^{\prime}(x)=0$ if $F(x)$ is the function to be optimized. Differential equations are very much used throughout science and engineering, and actually most engineering problems are optimization problems in the end, because one wants a design that maximizes performance and minimizes cost.

We first consider one algebraic equation in one variable, with our usual emphasis on how to program the algorithms. Systems of nonlinear algebraic equations with many variables arise from implicit methods for ordinary and partial differential equations as well as in multivariate optimization. Our attention will be restricted to Newton's method for such systems of nonlinear algebraic equations.

\section{Terminology}

When solving algebraic equations $f(x)=0$, we often say that the solution $x$ is a root of the equation. The solution process itself is thus often called root finding.

\subsection{Brute Force Methods}

The representation of a mathematical function $f(x)$ on a computer takes two forms. One is a Matlab function returning the function value given the argument, while the other is a collection of points $(x, f(x))$ along the function curve. The latter is the representation we use for plotting, together with an assumption of linear variation between the points. This representation is also very suited for equation solving and optimization: we simply go through all points and see if the function crosses the $x$ axis, or for optimization, test for a local maximum or minimum point. Because there is a lot of work to examine a huge number of points, and also because the idea is extremely simple, such approaches are often referred to as brute force methods. However, we are not embarrassed of explaining the methods in detail and implementing them. 


\subsubsection{Brute Force Root Finding}

Assume that we have a set of points along the curve of a function $f(x)$ :

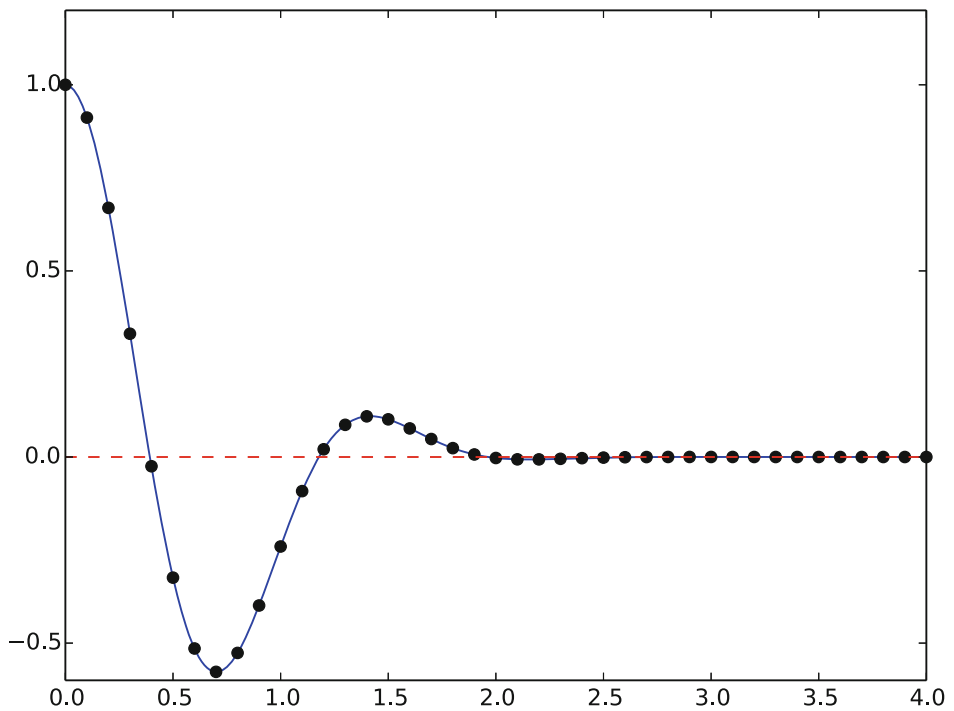

We want to solve $f(x)=0$, i.e., find the points $x$ where $f$ crosses the $x$ axis. A brute force algorithm is to run through all points on the curve and check if one point is below the $x$ axis and if the next point is above the $x$ axis, or the other way around. If this is found to be the case, we know that $f$ must be zero in between these two $x$ points.

Numerical algorithm More precisely, we have a set of $n+1$ points $\left(x_{i}, y_{i}\right), y_{i}=$ $f\left(x_{i}\right), i=0, \ldots, n$, where $x_{0}<\ldots<x_{n}$. We check if $y_{i}<0$ and $y_{i+1}>0$ (or the other way around). A compact expression for this check is to perform the test $y_{i} y_{i+1}<0$. If so, the root of $f(x)=0$ is in $\left[x_{i}, x_{i+1}\right]$. Assuming a linear variation of $f$ between $x_{i}$ and $x_{i+1}$, we have the approximation

$$
f(x) \approx \frac{f\left(x_{i+1}\right)-f\left(x_{i}\right)}{x_{i+1}-x_{i}}\left(x-x_{i}\right)+f\left(x_{i}\right)=\frac{y_{i+1}-y_{i}}{x_{i+1}-x_{i}}\left(x-x_{i}\right)+y_{i},
$$

which, when set equal to zero, gives the root

$$
x=x_{i}-\frac{x_{i+1}-x_{i}}{y_{i+1}-y_{i}} y_{i} .
$$

Implementation Given some Matlab implementation $f(x)$ of our mathematical function, a straightforward implementation of the above numerical algorithm looks like

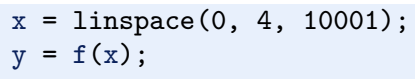




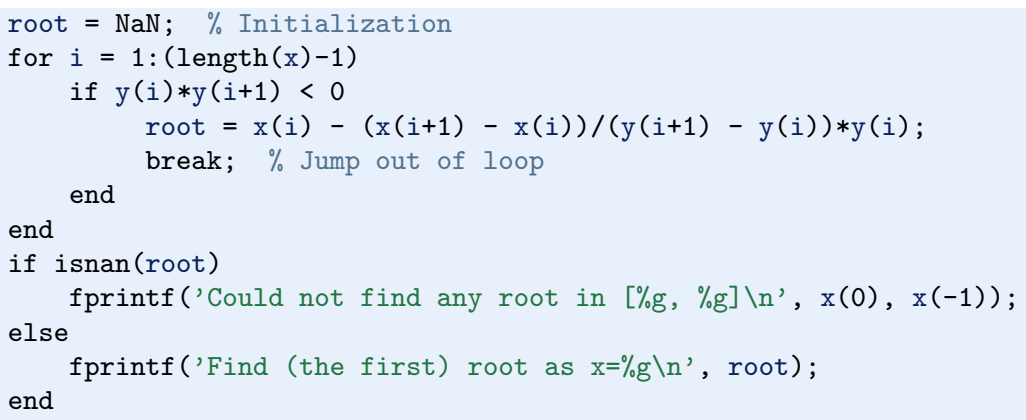

(See the file brute_force_root_finder_flat.m.)

Note the nice use of setting root to NaN: we can simply test if isnan (root) to see if we found a root and overwrote the $\mathrm{NaN}$ value, or if we did not find any root among the tested points.

Running this program with some function, say $f(x)=e^{-x^{2}} \cos (4 x)$ (which has a solution at $x=\frac{\pi}{8}$ ), gives the root 0.392699 , which has an error of $8.2 \cdot 10^{-8}$. Increasing the number of points with a factor of ten gives a root with an error of $3.1 \cdot 10^{-10}$.

After such a quick "flat" implementation of an algorithm, we should always try to offer the algorithm as a Matlab function, applicable to as wide a problem domain as possible. The function should take $f$ and an associated interval $[a, b]$ as input, as well as a number of points $(n)$, and return a list of all the roots in $[a, b]$. Here is our candidate for a good implementation of the brute force rooting finding algorithm:

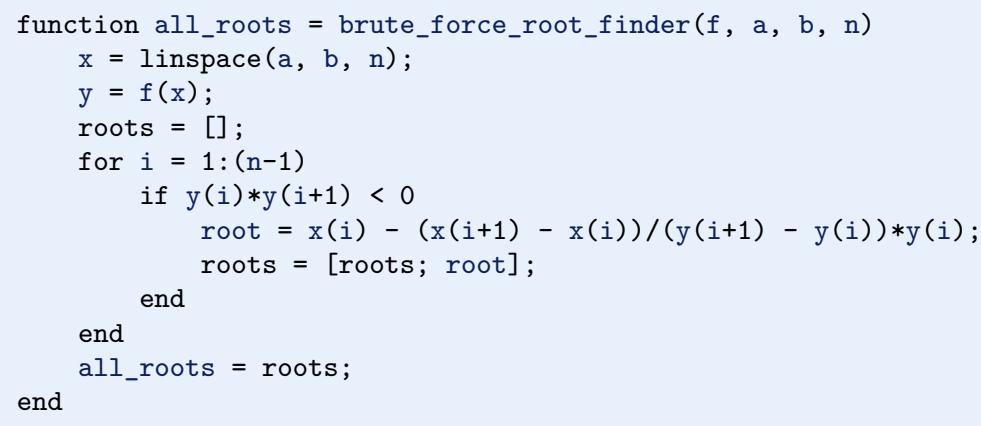

This function is found in the file brute_force_root_finder.m.

This time we use another elegant technique to indicate if roots were found or not: roots is empty (an array of length zero) if the root finding was unsuccessful, otherwise it contains all the roots. Application of the function to the previous example can be coded as (demo_brute_force_root_finder.m):

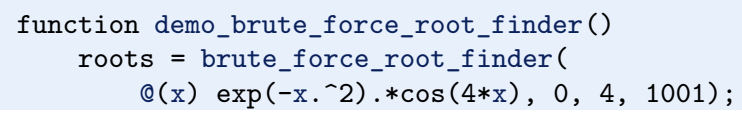




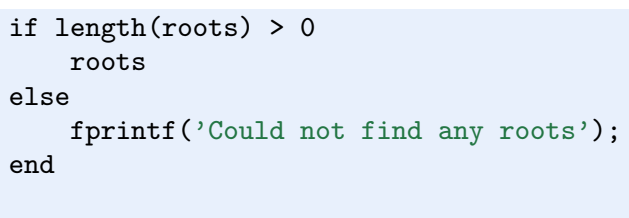

\subsubsection{Brute Force Optimization}

Numerical algorithm We realize that $x_{i}$ corresponds to a maximum point if $y_{i-1}<y_{i}>y_{i+1}$. Similarly, $x_{i}$ corresponds to a minimum if $y_{i-1}>y_{i}<y_{i+1}$. We can do this test for all "inner" points $i=1, \ldots, n-1$ to find all local minima and maxima. In addition, we need to add an end point, $i=0$ or $i=n$, if the corresponding $y_{i}$ is a global maximum or minimum.

Implementation The algorithm above can be translated to the following Matlab function (file brute_force_optimizer.m):

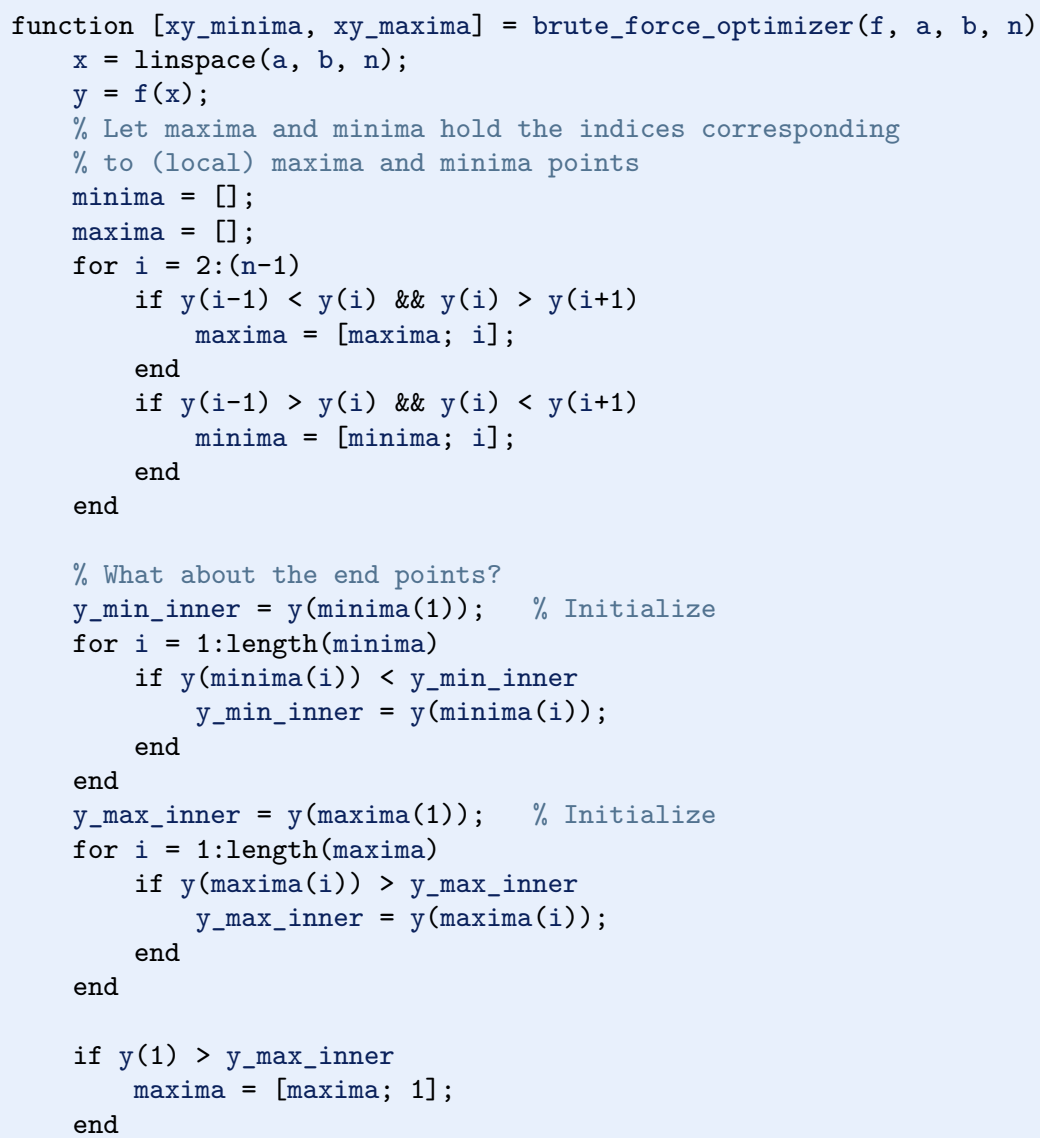




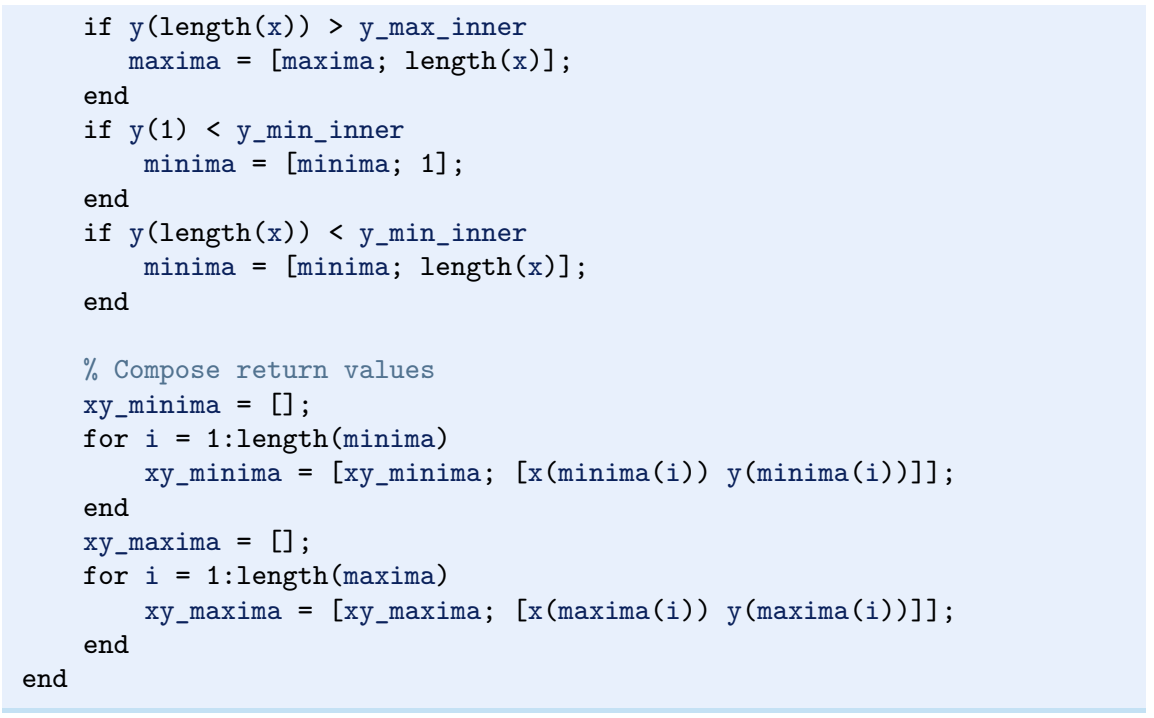

An application to $f(x)=e^{-x^{2}} \cos (4 x)$ looks like

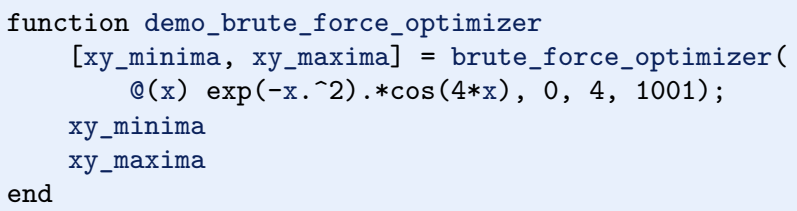

\subsubsection{Model Problem for Algebraic Equations}

We shall consider the very simple problem of finding the square root of 9, which is the positive solution of $x^{2}=9$. The nice feature of solving an equation whose solution is known beforehand is that we can easily investigate how the numerical method and the implementation perform in the search for the solution. The $f(x)$ function corresponding to the equation $x^{2}=9$ is

$$
f(x)=x^{2}-9 .
$$

Our interval of interest for solutions will be $[0,1000]$ (the upper limit here is chosen somewhat arbitrarily).

In the following, we will present several efficient and accurate methods for solving nonlinear algebraic equations, both single equation and systems of equations. The methods all have in common that they search for approximate solutions. The methods differ, however, in the way they perform the search for solutions. The idea for the search influences the efficiency of the search and the reliability of actually finding a solution. For example, Newton's method is very fast, but not reliable, while the bisection method is the slowest, but absolutely reliable. No method is best at all problems, so we need different methods for different problems. 


\section{What is the difference between linear and nonlinear equations?}

You know how to solve linear equations $a x+b=0: x=-b / a$. All other types of equations $f(x)=0$, i.e., when $f(x)$ is not a linear function of $x$, are called nonlinear. A typical way of recognizing a nonlinear equation is to observe that $x$ is "not alone" as in $a x$, but involved in a product with itself, such as in $x^{3}+2 x^{2}-9=0$. We say that $x^{3}$ and $2 x^{2}$ are nonlinear terms. An equation like $\sin x+e^{x} \cos x=0$ is also nonlinear although $x$ is not explicitly multiplied by itself, but the Taylor series of $\sin x, e^{x}$, and $\cos x$ all involve polynomials of $x$ where $x$ is multiplied by itself.

\subsection{Newton's Method}

Newton's method, also known as Newton-Raphson's method, is a very famous and widely used method for solving nonlinear algebraic equations. Compared to the other methods we will consider, it is generally the fastest one (usually by far). It does not guarantee that an existing solution will be found, however.

A fundamental idea of numerical methods for nonlinear equations is to construct a series of linear equations (since we know how to solve linear equations) and hope that the solutions of these linear equations bring us closer and closer to the solution of the nonlinear equation. The idea will be clearer when we present Newton's method and the secant method.

\subsubsection{Deriving and Implementing Newton's Method}

Figure 6.1 shows the $f(x)$ function in our model equation $x^{2}-9=0$. Numerical methods for algebraic equations require us to guess at a solution first. Here, this guess is called $x_{0}$. The fundamental idea of Newton's method is to approximate the original function $f(x)$ by a straight line, i.e., a linear function, since it is straightforward to solve linear equations. There are infinitely many choices of how to approximate $f(x)$ by a straight line. Newton's method applies the tangent of $f(x)$ at $x_{0}$, see the rightmost tangent in Fig. 6.1. This linear tangent function crosses the $x$ axis at a point we call $x_{1}$. This is (hopefully) a better approximation to the solution of $f(x)=0$ than $x_{0}$. The next fundamental idea is to repeat this process. We find the tangent of $f$ at $x_{1}$, compute where it crosses the $x$ axis, at a point called $x_{2}$, and repeat the process again. Figure 6.1 shows that the process brings us closer and closer to the left. It remains, however, to see if we hit $x=3$ or come sufficiently close to this solution.

How do we compute the tangent of a function $f(x)$ at a point $x_{0}$ ? The tangent function, here called $\tilde{f}(x)$, is linear and has two properties:

1. the slope equals to $f^{\prime}\left(x_{0}\right)$

2. the tangent touches the $f(x)$ curve at $x_{0}$ 


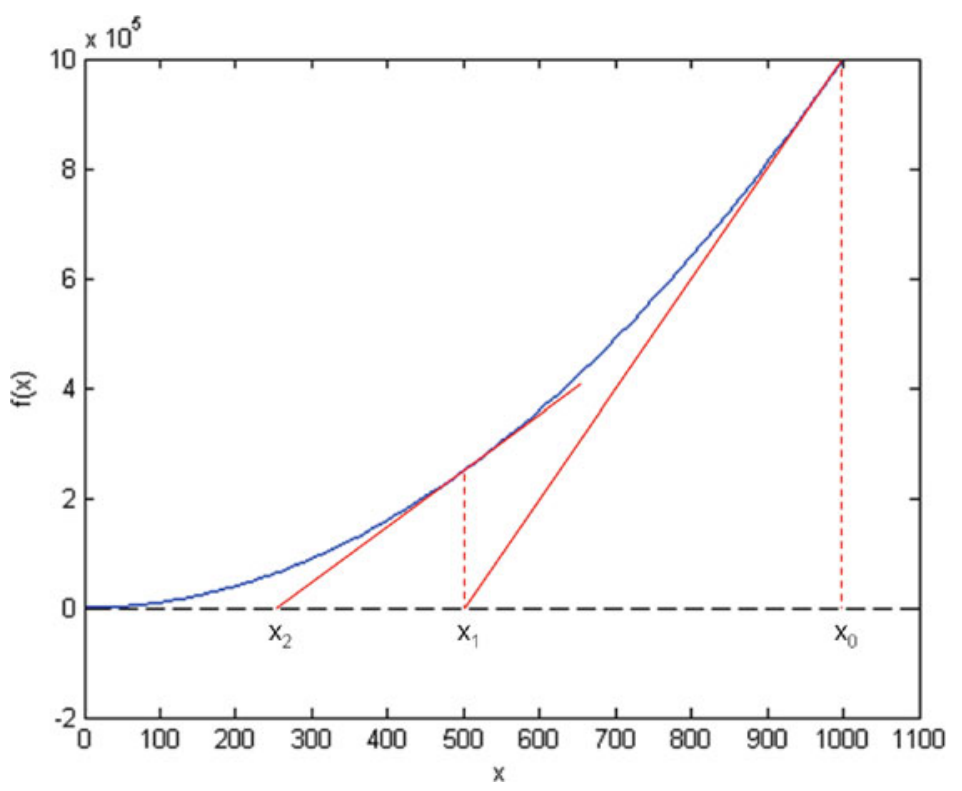

Fig. 6.1 Illustrates the idea of Newton's method with $f(x)=x^{2}-9$, repeatedly solving for crossing of tangent lines with the $x$ axis

So, if we write the tangent function as $\tilde{f}(x)=a x+b$, we must require $\tilde{f}^{\prime}\left(x_{0}\right)=$ $f^{\prime}\left(x_{0}\right)$ and $\tilde{f}\left(x_{0}\right)=f\left(x_{0}\right)$, resulting in

$$
\tilde{f}(x)=f\left(x_{0}\right)+f^{\prime}\left(x_{0}\right)\left(x-x_{0}\right) .
$$

The key step in Newton's method is to find where the tangent crosses the $x$ axis, which means solving $\tilde{f}(x)=0$ :

$$
\tilde{f}(x)=0 \Rightarrow x=x_{0}-\frac{f\left(x_{0}\right)}{f^{\prime}\left(x_{0}\right)} .
$$

This is our new candidate point, which we call $x_{1}$ :

$$
x_{1}=x_{0}-\frac{f\left(x_{0}\right)}{f^{\prime}\left(x_{0}\right)} .
$$

With $x_{0}=1000$, we get $x_{1} \approx 500$, which is in accordance with the graph in Fig. 6.1. Repeating the process, we get

$$
x_{2}=x_{1}-\frac{f\left(x_{1}\right)}{f^{\prime}\left(x_{1}\right)} \approx 250 .
$$

The general scheme of Newton's method may be written as

$$
x_{n+1}=x_{n}-\frac{f\left(x_{n}\right)}{f^{\prime}\left(x_{n}\right)}, \quad n=0,1,2, \ldots
$$


The computation in (6.1) is repeated until $f\left(x_{n}\right)$ is close enough to zero. More precisely, we test if $\left|f\left(x_{n}\right)\right|<\epsilon$, with $\epsilon$ being a small number.

We moved from 1000 to 250 in two iterations, so it is exciting to see how fast we can approach the solution $x=3$. A computer program can automate the calculations. Our first try at implementing Newton's method is in a function naive_Newton:

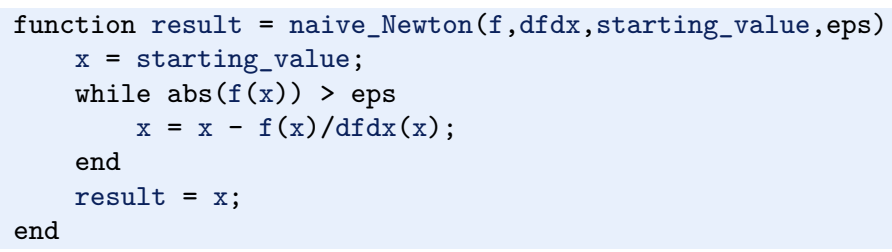

The argument $\mathrm{x}$ is the starting value, called $x_{0}$ in our previous description. To solve the problem $x^{2}=9$ we also need to implement

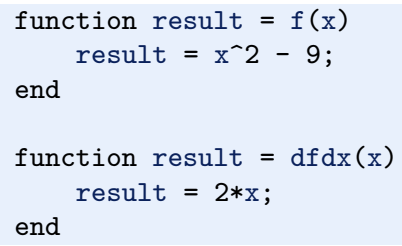

\section{Why not use an array for the $x$ approximations?}

Newton's method is normally formulated with an iteration index $n$,

$$
x_{n+1}=x_{n}-\frac{f\left(x_{n}\right)}{f^{\prime}\left(x_{n}\right)} \text {. }
$$

Seeing such an index, many would implement this as

$x(n+1)=x(x)-f(x(n)) / d f d x(x(n)) ;$

Such an array is fine, but requires storage of all the approximations. In large industrial applications, where Newton's method solves millions of equations at once, one cannot afford to store all the intermediate approximations in memory, so then it is important to understand that the algorithm in Newton's method has no more need for $x_{n}$ when $x_{n+1}$ is computed. Therefore, we can work with one variable $\mathrm{x}$ and overwrite the previous value:

$x=x-f(x) / d f d x(x)$

Running naive_Newton( $f, \mathrm{dfdx}, 1000$, eps $=0.001$ ) results in the approximate solution 3.000027639. A smaller value of eps will produce a more accurate solution. Unfortunately, the plain naive_Newton function does not return how many iterations it used, nor does it print out all the approximations 
$x_{0}, x_{1}, x_{2}, \ldots$, which would indeed be a nice feature. If we insert such a printout, a rerun results in
500.0045
250.011249919
125.02362415
62.5478052723
31.3458476066
15.816483488
8.1927550496
4.64564330569
3. 2914711388
3. 01290538807
3. 00002763928

We clearly see that the iterations approach the solution quickly. This speed of the search for the solution is the primary strength of Newton's method compared to other methods.

\subsubsection{Making a More Efficient and Robust Implementation}

The naive_Newton function works fine for the example we are considering here. However, for more general use, there are some pitfalls that should be fixed in an improved version of the code. An example may illustrate what the problem is: let us solve $\tanh (x)=0$, which has solution $x=0$. With $\left|x_{0}\right| \leq 1.08$ everything works fine. For example, $x_{0}$ leads to six iterations if $\epsilon=0.001$ :
$-1.05895313436$
0.989404207298
$-0.784566773086$
0.36399816111
$-0.0330146961372$
$2.3995252668 e-05$

Adjusting $x_{0}$ slightly to 1.09 gives division by zero! The approximations computed by Newton's method become
$-1.09331618202$
1.10490354324
$-1.14615550788$
1.30303261823
$-2.06492300238$
13.4731428006
$-1.26055913647 e+11$

The division by zero is caused by $x_{7}=-1.26055913647 \cdot 10^{11}$, because $\tanh \left(x_{7}\right)$ is 1.0 to machine precision, and then $f^{\prime}(x)=1-\tanh (x)^{2}$ becomes zero in the denominator in Newton's method.

The underlying problem, leading to the division by zero in the above example, is that Newton's method diverges: the approximations move further and further 
away from $x=0$. If it had not been for the division by zero, the condition in the while loop would always be true and the loop would run forever. Divergence of Newton's method occasionally happens, and the remedy is to abort the method when a maximum number of iterations is reached.

Another disadvantage of the naive_Newton function is that it calls the $f(x)$ function twice as many times as necessary. This extra work is of no concern when $f(x)$ is fast to evaluate, but in large-scale industrial software, one call to $f(x)$ might take hours or days, and then removing unnecessary calls is important. The solution in our function is to store the call $f(x)$ in a variable ( $f_{-}$value) and reuse the value instead of making a new call $f(x)$.

To summarize, we want to write an improved function for implementing Newton's method where we

- avoid division by zero

- allow a maximum number of iterations

- avoid the extra evaluation of $f(x)$

A more robust and efficient version of the function, inserted in a complete program Newtons_method.m for solving $x^{2}-9=0$, is listed below.

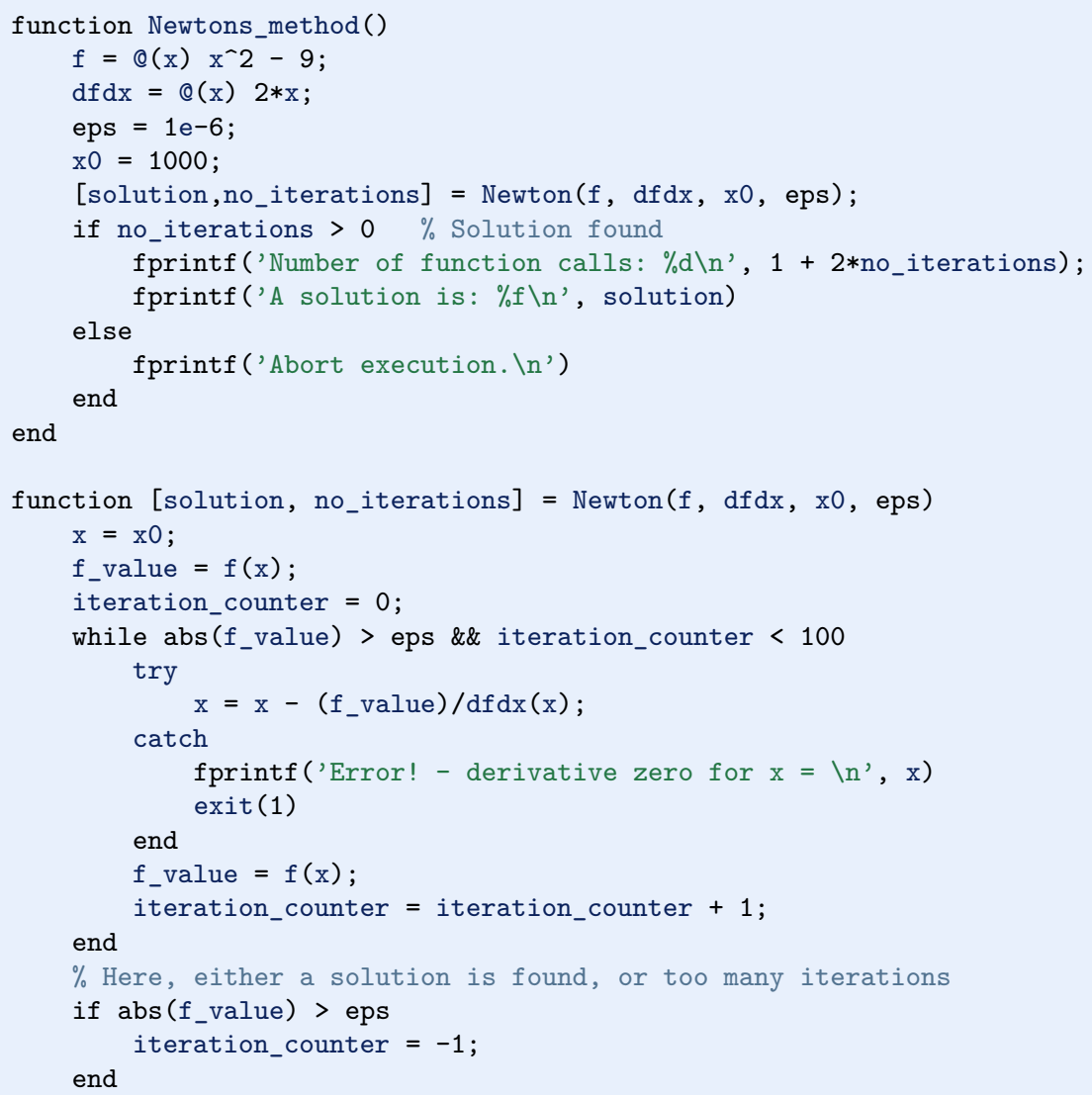


solution $=\mathrm{x}$;

no_iterations = iteration_counter;

end

Handling of the potential division by zero is done by a try-catch construction, which works as follows. First, Matlab tries to execute the code in the try block, but if something goes wrong there, the catch block is executed instead and the execution is terminated by exit.

The division by zero will always be detected and the program will be stopped. The main purpose of our way of treating the division by zero is to give the user a more informative error message and stop the program in a gentler way.

Calling exit with an argument different from zero (here 1) signifies that the program stopped because of an error. It is a good habit to supply the value 1, because tools in the operating system can then be used by other programs to detect that our program failed.

To prevent an infinite loop because of divergent iterations, we have introduced the integer variable iteration_counter to count the number of iterations in Newton's method. With iteration_counter we can easily extend the condition in the while such that no more iterations take place when the number of iterations reaches 100. We could easily let this limit be an argument to the function rather than a fixed constant.

The Newton function returns the approximate solution and the number of iterations. The latter equals -1 if the convergence criterion $|f(x)|<\epsilon$ was not reached within the maximum number of iterations. In the calling code, we print out the solution and the number of function calls. The main cost of a method for solving $f(x)=0$ equations is usually the evaluation of $f(x)$ and $f^{\prime}(x)$, so the total number of calls to these functions is an interesting measure of the computational work. Note that in function Newton there is an initial call to $f(x)$ and then one call to $f$ and one to $f^{\prime}$ in each iteration.

Running Newtons_method.m, we get the following printout on the screen:

Number of function calls: 25

A solution is: 3.000000

As we did with the integration methods in Chap. 3, we will place our solvers for nonlinear algebraic equations in separate files for easy use by other programs. So, we place Newton in the file Newton.m

The Newton scheme will work better if the starting value is close to the solution. A good starting value may often make the difference as to whether the code actually finds a solution or not. Because of its speed, Newton's method is often the method of first choice for solving nonlinear algebraic equations, even if the scheme is not guaranteed to work. In cases where the initial guess may be far from the solution, a good strategy is to run a few iterations with the bisection method (see Sect. 6.4) to narrow down the region where $f$ is close to zero and then switch to Newton's method for fast convergence to the solution.

Newton's method requires the analytical expression for the derivative $f^{\prime}(x)$. Derivation of $f^{\prime}(x)$ is not always a reliable process by hand if $f(x)$ is a complicated function. However, Matlab has the Symbolic Math Toolbox, which we may use to 
create the required $d f d x$ function (Octave does not (yet) offer the same possibilities for symbolic computations as Matlab. However, there is work in progress, e.g. on using SymPy (from Python) from Octave). In our sample problem, the recipe goes as follows:

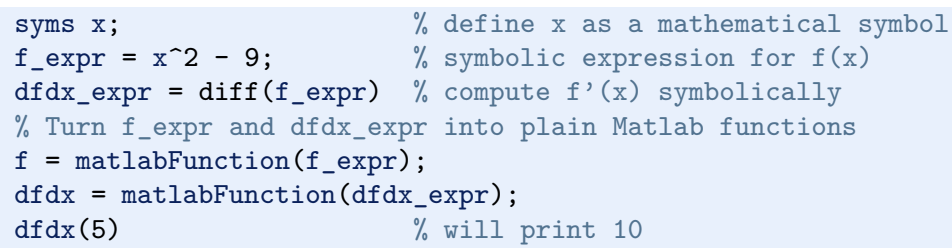

The nice feature of this code snippet is that $d f d x_{-}$expr is the exact analytical expression for the derivative, $2 * \mathrm{x}$, if you print it out. This is a symbolic expression so we cannot do numerical computing with it, but the matlabFunction turns symbolic expressions into callable Matlab functions.

The next method is the secant method, which is usually slower than Newton's method, but it does not require an expression for $f^{\prime}(x)$, and it has only one function call per iteration.

\subsection{The Secant Method}

When finding the derivative $f^{\prime}(x)$ in Newton's method is problematic, or when function evaluations take too long; we may adjust the method slightly. Instead of using tangent lines to the graph we may use secants ${ }^{1}$. The approach is referred to as the secant method, and the idea is illustrated graphically in Fig. 6.2 for our example problem $x^{2}-9=0$.

The idea of the secant method is to think as in Newton's method, but instead of using $f^{\prime}\left(x_{n}\right)$, we approximate this derivative by a finite difference or the $s e$ cant, i.e., the slope of the straight line that goes through the points $\left(x_{n}, f\left(x_{n}\right)\right)$ and $\left(x_{n-1}, f\left(x_{n-1}\right)\right)$ on the graph, given by the two most recent approximations $x_{n}$ and $x_{n-1}$. This slope reads

$$
\frac{f\left(x_{n}\right)-f\left(x_{n-1}\right)}{x_{n}-x_{n-1}} .
$$

Inserting this expression for $f^{\prime}\left(x_{n}\right)$ in Newton's method simply gives us the secant method:

$$
x_{n+1}=x_{n}-\frac{f\left(x_{n}\right)}{\frac{f\left(x_{n}\right)-f\left(x_{n-1}\right)}{x_{n}-x_{n-1}}},
$$

or

$$
x_{n+1}=x_{n}-f\left(x_{n}\right) \frac{x_{n}-x_{n-1}}{f\left(x_{n}\right)-f\left(x_{n-1}\right)} .
$$

Comparing (6.3) to the graph in Fig. 6.2, we see how two chosen starting points ( $x_{0}=1000, x_{1}=700$, and corresponding function values) are used to compute $x_{2}$. Once we have $x_{2}$, we similarly use $x_{1}$ and $x_{2}$ to compute $x_{3}$. As with Newton's

\footnotetext{
${ }^{1}$ https://en.wikipedia.org/wiki/Secant_line
} 


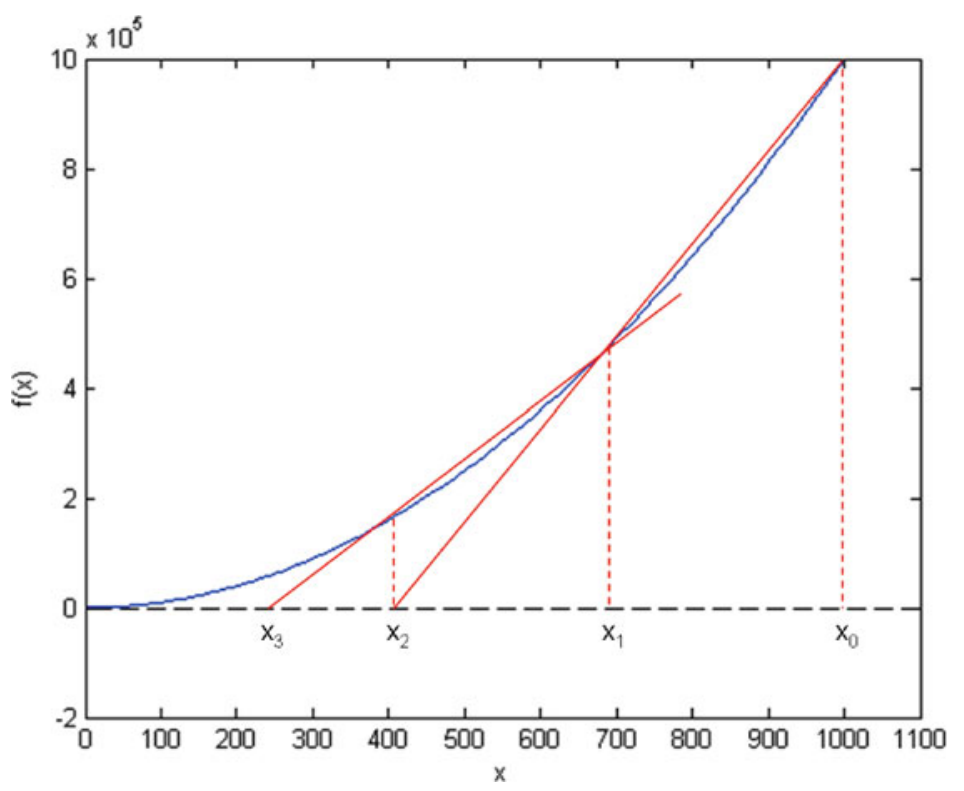

Fig.6.2 Illustrates the use of secants in the secant method when solving $x^{2}-9=0, x \in[0,1000]$. From two chosen starting values, $x_{0}=1000$ and $x_{1}=700$ the crossing $x_{2}$ of the corresponding secant with the $x$ axis is computed, followed by a similar computation of $x_{3}$ from $x_{1}$ and $x_{2}$

method, the procedure is repeated until $f\left(x_{n}\right)$ is below some chosen limit value, or some limit on the number of iterations has been reached. We use an iteration counter here too, based on the same thinking as in the implementation of Newton's method.

We can store the approximations $x_{n}$ in an array, but as in Newton's method, we notice that the computation of $x_{n+1}$ only needs knowledge of $x_{n}$ and $x_{n-1}$, not "older" approximations. Therefore, we can make use of only three variables: $\mathrm{x}$ for $x_{n+1}, \mathrm{x} 1$ for $x_{n}$, and $\mathrm{x} 0$ for $x_{n-1}$. Note that $\mathrm{x} 0$ and $\mathrm{x} 1$ must be given (guessed) for the algorithm to start.

A program secant_method.m that solves our example problem may be written as:

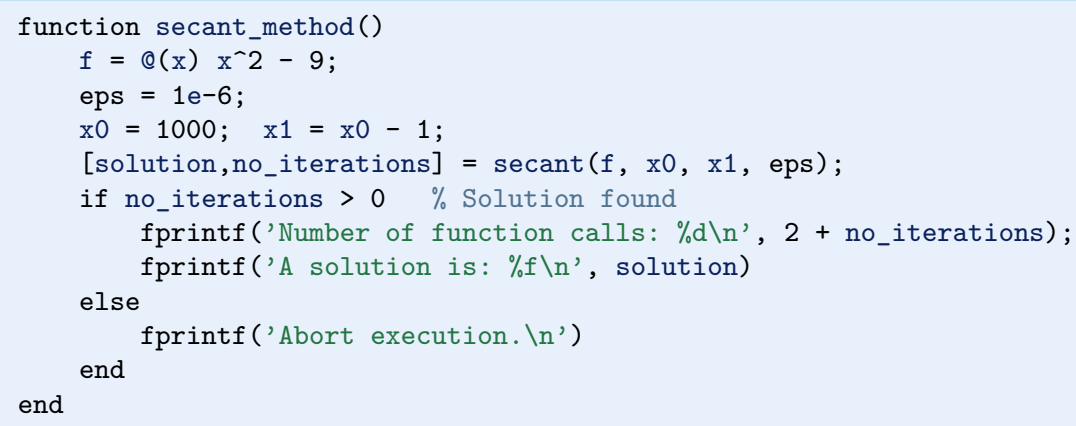




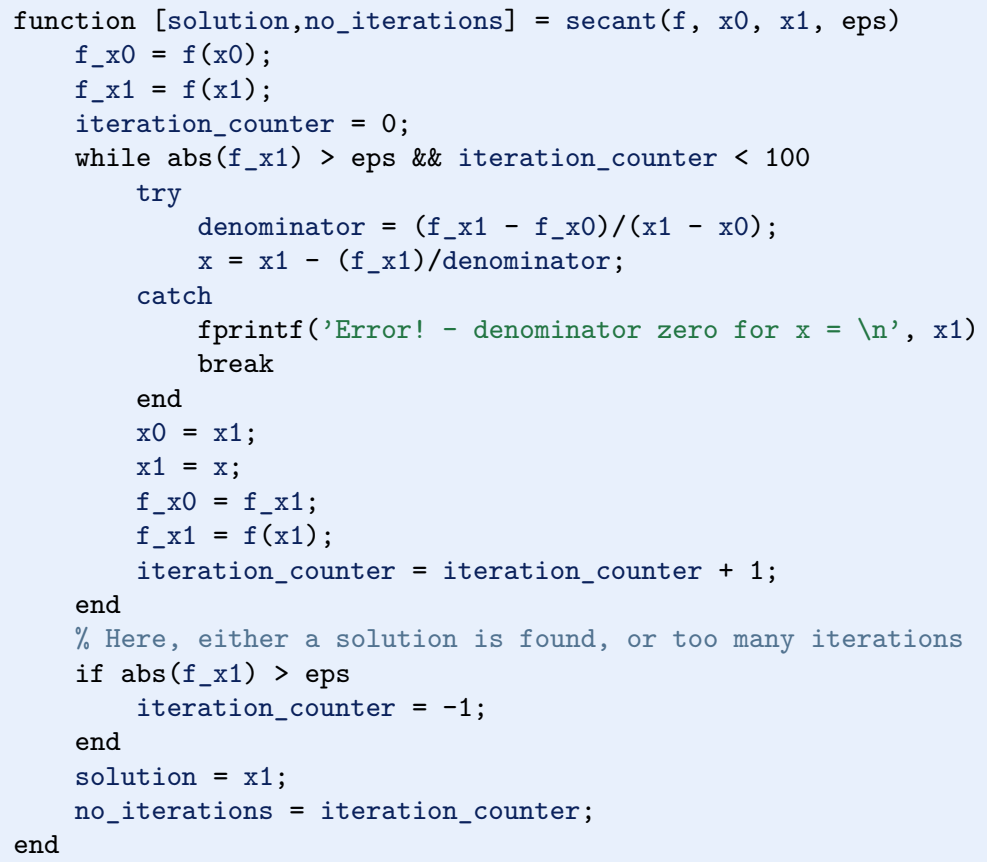

The number of function calls is now related to no_iterations, i.e., the number of iterations, as $2+$ no_iterations, since we need two function calls before entering the while loop, and then one function call per loop iteration. Note that, even though we need two points on the graph to compute each updated estimate, only a single function call $(f(x 1))$ is required in each iteration since $f(x 0)$ becomes the "old" $f(x 1)$ and may simply be copied as $f_{-} x 0=f_{-} x 1$ (the exception is the very first iteration where two function evaluations are needed).

Running secant_method.m, gives the following printout on the screen:

Number of function calls: 19

A solution is: 3.000000

As with the function Newton, we place secant in a separate file secant.m for easy use later.

\subsection{The Bisection Method}

Neither Newton's method nor the secant method can guarantee that an existing solution will be found (see Exercises 6.1 and 6.2). The bisection method, however, does that. However, if there are several solutions present, it finds only one of them, just as Newton's method and the secant method. The bisection method is slower than the other two methods, so reliability comes with a cost of speed.

To solve $x^{2}-9=0, x \in[0,1000]$, with the bisection method, we reason as follows. The first key idea is that if $f(x)=x^{2}-9$ is continuous on the interval and 
the function values for the interval endpoints $\left(x_{L}=0, x_{R}=1000\right)$ have opposite signs, $f(x)$ must cross the $x$ axis at least once on the interval. That is, we know there is at least one solution.

The second key idea comes from dividing the interval in two equal parts, one to the left and one to the right of the midpoint $x_{M}=500$. By evaluating the sign of $f\left(x_{M}\right)$, we will immediately know whether a solution must exist to the left or right of $x_{M}$. This is so, since if $f\left(x_{M}\right) \geq 0$, we know that $f(x)$ has to cross the $x$ axis between $x_{L}$ and $x_{M}$ at least once (using the same argument as for the original interval). Likewise, if instead $f\left(x_{M}\right) \leq 0$, we know that $f(x)$ has to cross the $x$ axis between $x_{M}$ and $x_{R}$ at least once.

In any case, we may proceed with half the interval only. The exception is if $f\left(x_{M}\right) \approx 0$, in which case a solution is found. Such interval halving can be continued until a solution is found. A "solution" in this case, is when $\left|f\left(x_{M}\right)\right|$ is sufficiently close to zero, more precisely (as before): $\left|f\left(x_{M}\right)\right|<\epsilon$, where $\epsilon$ is a small number specified by the user.

The sketched strategy seems reasonable, so let us write a reusable function that can solve a general algebraic equation $f(x)=0$ (bisection_method.m):

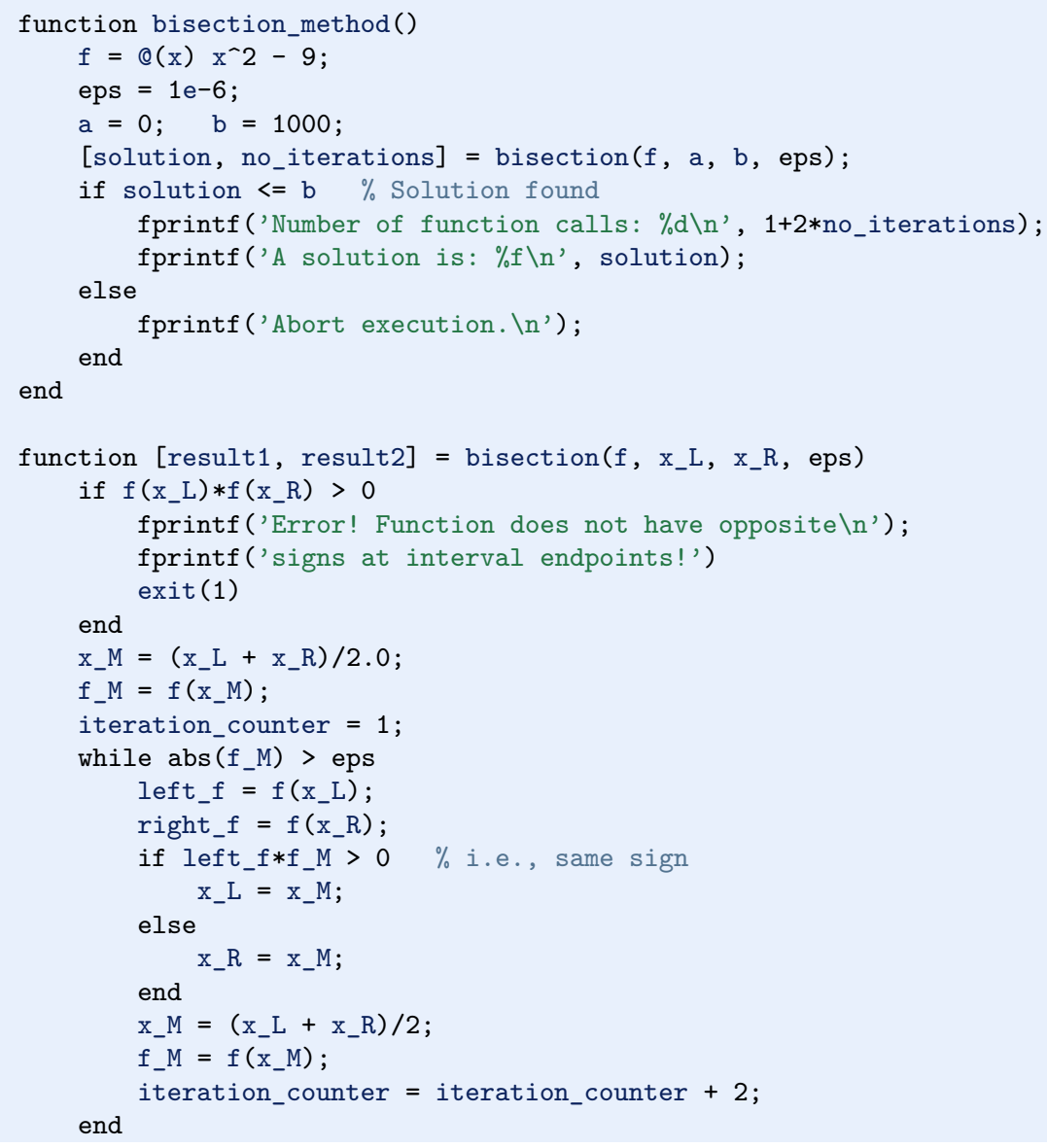




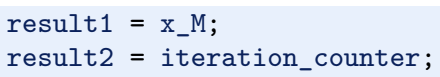

Note that we first check if $f$ changes sign in $[a, b]$, because that is a requirement for the algorithm to work. The algorithm also relies on a continuous $f(x)$ function, but this is very challenging for a computer code to check.

We get the following printout to the screen when bisection_method.m is run:

Number of function calls: 61

A solution is: 3.000000

We notice that the number of function calls is much higher than with the previous methods.

\section{Required work in the bisection method}

If the starting interval of the bisection method is bounded by $a$ and $b$, and the solution at step $n$ is taken to be the middle value, the error is bounded as

$$
\frac{|b-a|}{2^{n}}
$$

because the initial interval has been halved $n$ times. Therefore, to meet a tolerance $\epsilon$, we need $n$ iterations such that the length of the current interval equals $\epsilon$ :

$$
\frac{|b-a|}{2^{n}}=\epsilon \quad \Rightarrow \quad n=\frac{\ln ((b-a) / \epsilon)}{\ln 2} .
$$

This is a great advantage of the bisection method: we know beforehand how many iterations $n$ it takes to meet a certain accuracy $\epsilon$ in the solution.

As with the two previous methods, the function bisection is stored as a separate file bisection.m for easy use by other programs.

\subsection{Rate of Convergence}

With the methods above, we noticed that the number of iterations or function calls could differ quite substantially. The number of iterations needed to find a solution is closely related to the rate of convergence, which dictates the speed of error reduction as we approach the root. More precisely, we introduce the error in iteration $n$ as $e_{n}=\left|x-x_{n}\right|$, and define the convergence rate $q$ as

$$
e_{n+1}=C e_{n}^{q},
$$

where $C$ is a constant. The exponent $q$ measures how fast the error is reduced from one iteration to the next. The larger $q$ is, the faster the error goes to zero, and the fewer iterations we need to meet the stopping criterion $|f(x)|<\epsilon$. 
A single $q$ in (6.5) is defined in the limit $n \rightarrow \infty$. For finite $n$, and especially smaller $n, q$ will vary with $n$. To estimate $q$, we can compute all the errors $e_{n}$ and set up (6.5) for three consecutive experiments $n-1, n$, and $n+1$ :

$$
\begin{aligned}
e_{n} & =C e_{n-1}^{q}, \\
e_{n+1} & =C e_{n}^{q} .
\end{aligned}
$$

Dividing these two equations by each other and solving with respect to $q$ gives

$$
q=\frac{\ln \left(e_{n+1} / e_{n}\right)}{\ln \left(e_{n} / e_{n-1}\right)} .
$$

Since this $q$ will vary somewhat with $n$, we call it $q_{n}$. As $n$ grows, we expect $q_{n}$ to approach a limit $\left(q_{n} \rightarrow q\right)$. To compute all the $q_{n}$ values, we need all the $x_{n}$ approximations. However, our previous implementations of Newton's method, the secant method, and the bisection method returned just the final approximation.

Therefore, we have extended those previous implementations such that the user can choose whether the final value or the whole history of solutions is to be returned. The extended implementations are named Newton_solver, secant_solver and bisection_solver. Compared to the previous implementations, each of these now takes an extra parameter return_x_list. This parameter is a boolean, set to true if the function is supposed to return all the root approximations, or false, if the function should only return the final approximation. As an example, let us take a closer look at Newton_solver:

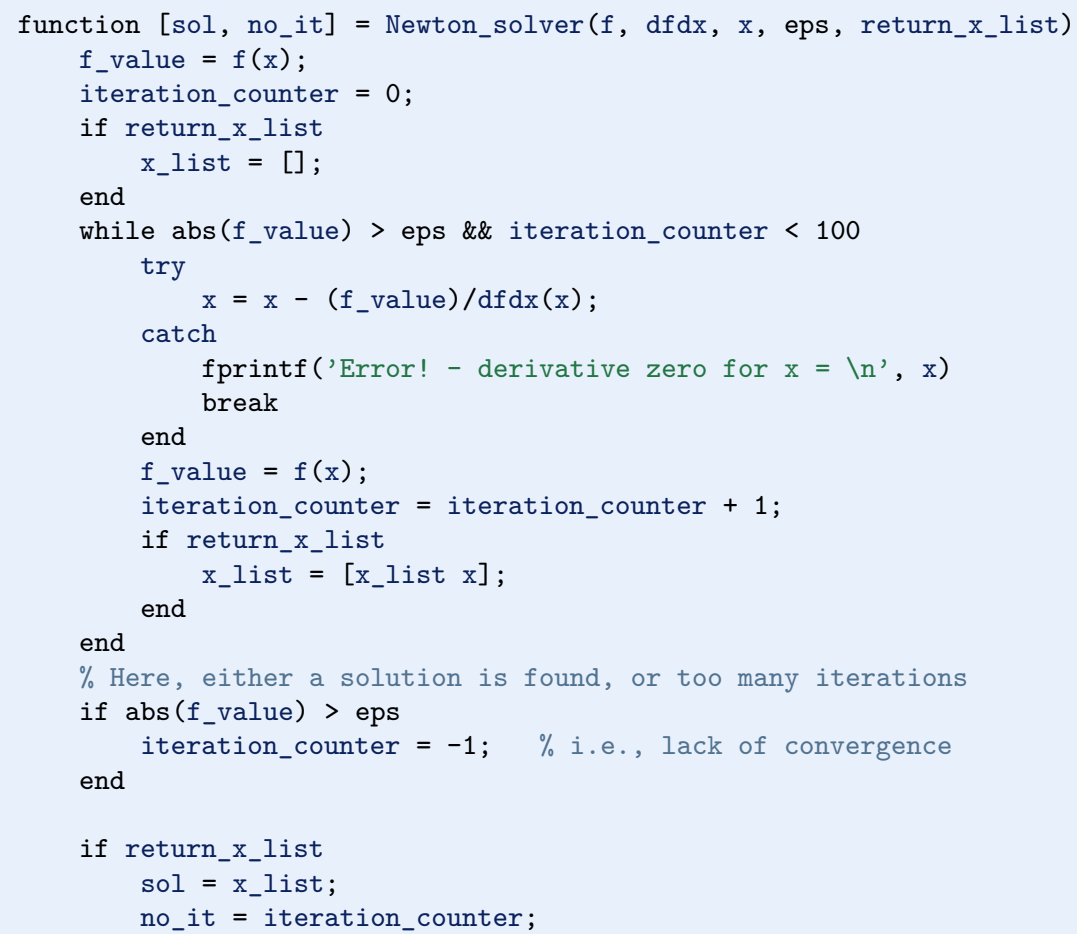


else

sol $=\mathrm{x}$;

end

no_it = iteration_counter;

end

The function is found in the file Newton_solver.m.

We can now make a call

$[x$, iter $]=$ Newton_solver $(f, d f d x, 1000,1 e-6$, true $)$;

and get an array $\mathrm{x}$ returned. With knowledge of the exact solution $x$ of $f(x)=0$, we can compute all the errors $e_{n}$ and associated $q_{n}$ values with the compact function

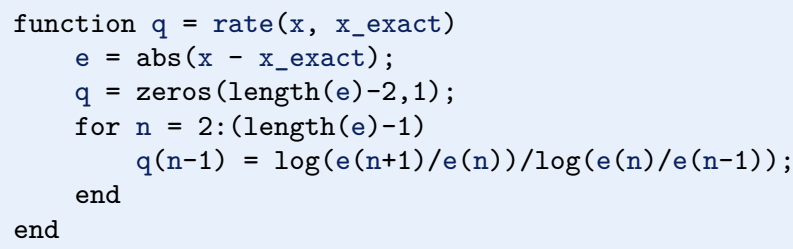

The error model (6.5) works well for Newton's method and the secant method. For the bisection method, however, it works well in the beginning, but not when the solution is approached.

We can compute the rates $q_{n}$ and print them nicely,

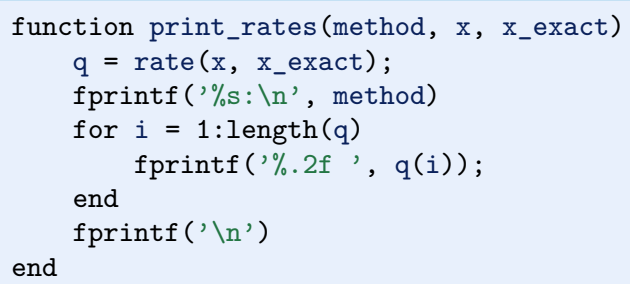

The result for print_rates ('Newton', $\mathrm{x}, 3$ ) is

\section{Newton:}

$\begin{array}{llllllllllll}1.01 & 1.02 & 1.03 & 1.07 & 1.14 & 1.27 & 1.51 & 1.80 & 1.97 & 2.00\end{array}$

indicating that $q=2$ is the rate for Newton's method. A similar computation using the secant method, gives the rates

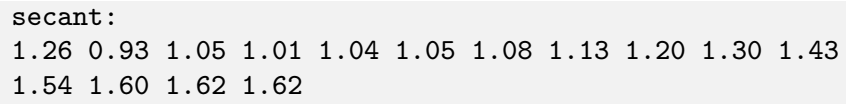

Here it seems that $q \approx 1.6$ is the limit. 
Remark If we in the bisection method think of the length of the current interval containing the solution as the error $e_{n}$, then (6.5) works perfectly since $e_{n+1}=$ $\frac{1}{2} e_{n}$, i.e., $q=1$ and $C=\frac{1}{2}$, but if $e_{n}$ is the true error $\left|x-x_{n}\right|$, it is easily seen from a sketch that this error can oscillate between the current interval length and a potentially very small value as we approach the exact solution. The corresponding rates $q_{n}$ fluctuate widely and are of no interest.

\subsection{Solving Multiple Nonlinear Algebraic Equations}

So far in this chapter, we have considered a single nonlinear algebraic equation. However, systems of such equations arise in a number of applications, foremost nonlinear ordinary and partial differential equations. Of the previous algorithms, only Newton's method is suitable for extension to systems of nonlinear equations.

\subsubsection{Abstract Notation}

Suppose we have $n$ nonlinear equations, written in the following abstract form:

$$
\begin{aligned}
F_{0}\left(x_{0}, x_{1}, \ldots, x_{n}\right) & =0 \\
F_{1}\left(x_{0}, x_{1}, \ldots, x_{n}\right) & =0 \\
\vdots & =\vdots \\
F_{n}\left(x_{0}, x_{1}, \ldots, x_{n}\right) & =0
\end{aligned}
$$

It will be convenient to introduce a vector notation

$$
\boldsymbol{F}=\left(F_{0}, \ldots, F_{1}\right), \quad \boldsymbol{x}=\left(x_{0}, \ldots, x_{n}\right)
$$

The system can now be written as $\boldsymbol{F}(\boldsymbol{x})=\mathbf{0}$.

As a specific example on the notation above, the system

$$
\begin{aligned}
x^{2} & =y-x \cos (\pi x) \\
y x+e^{-y} & =x^{-1}
\end{aligned}
$$

can be written in our abstract form by introducing $x_{0}=x$ and $x_{1}=y$. Then

$$
\begin{aligned}
& F_{0}\left(x_{0}, x_{1}\right)=x^{2}-y+x \cos (\pi x)=0, \\
& F_{1}\left(x_{0}, x_{1}\right)=y x+e^{-y}-x^{-1}=0 .
\end{aligned}
$$

\subsubsection{Taylor Expansions for Multi-Variable Functions}

We follow the ideas of Newton's method for one equation in one variable: approximate the nonlinear $f$ by a linear function and find the root of that function. When 
$n$ variables are involved, we need to approximate a vector function $\boldsymbol{F}(\boldsymbol{x})$ by some linear function $\tilde{\boldsymbol{F}}=\boldsymbol{J} \boldsymbol{x}+\boldsymbol{c}$, where $\boldsymbol{J}$ is an $n \times n$ matrix and $\boldsymbol{c}$ is some vector of length $n$.

The technique for approximating $\boldsymbol{F}$ by a linear function is to use the first two terms in a Taylor series expansion. Given the value of $\boldsymbol{F}$ and its partial derivatives with respect to $\boldsymbol{x}$ at some point $\boldsymbol{x}_{i}$, we can approximate the value at some point $\boldsymbol{x}_{i+1}$ by the two first term in a Taylor series expansion around $\boldsymbol{x}_{i}$ :

$$
\boldsymbol{F}\left(\boldsymbol{x}_{i+1}\right) \approx \boldsymbol{F}\left(\boldsymbol{x}_{i}\right)+\nabla \boldsymbol{F}\left(\boldsymbol{x}_{i}\right)\left(\boldsymbol{x}_{i+1}-\boldsymbol{x}_{i}\right) .
$$

The next terms in the expansions are omitted here and of size $\left\|\boldsymbol{x}_{i+1}-\boldsymbol{x}_{i}\right\|^{2}$, which are assumed to be small compared with the two terms above.

The expression $\nabla \boldsymbol{F}$ is the matrix of all the partial derivatives of $\boldsymbol{F}$. Component $(i, j)$ in $\nabla \boldsymbol{F}$ is

$$
\frac{\partial F_{i}}{\partial x_{j}}
$$

For example, in our $2 \times 2$ system (6.11)-(6.12) we can use SymPy to compute the Jacobian:

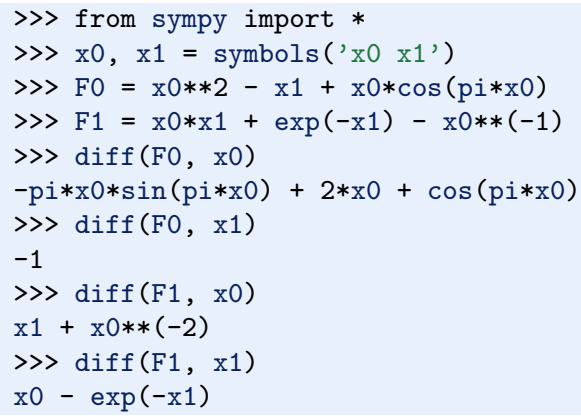

We can then write

$$
\nabla \boldsymbol{F}=\left(\begin{array}{ll}
\frac{\partial F_{0}}{\partial x_{0}} & \frac{\partial F_{0}}{\partial x_{1}} \\
\frac{\partial F_{1}}{\partial x_{0}} & \frac{\partial F_{1}}{\partial x_{1}}
\end{array}\right)=\left(\begin{array}{ll}
2 x_{0}+\cos \left(\pi x_{0}\right)-\pi x_{0} \sin \left(\pi x_{0}\right) & -1 \\
x_{1}+x_{0}^{-2} & x_{0}-e^{-x_{1}}
\end{array}\right)
$$

The matrix $\nabla \boldsymbol{F}$ is called the Jacobian of $\boldsymbol{F}$ and often denoted by $\boldsymbol{J}$.

\subsubsection{Newton's Method}

The idea of Newton's method is that we have some approximation $\boldsymbol{x}_{i}$ to the root and seek a new (and hopefully better) approximation $\boldsymbol{x}_{i+1}$ by approximating $\boldsymbol{F}\left(\boldsymbol{x}_{i+1}\right)$ by a linear function and solve the corresponding linear system of algebraic equations. We approximate the nonlinear problem $\boldsymbol{F}\left(\boldsymbol{x}_{i+1}\right)=0$ by the linear problem

$$
\boldsymbol{F}\left(\boldsymbol{x}_{i}\right)+\boldsymbol{J}\left(\boldsymbol{x}_{i}\right)\left(\boldsymbol{x}_{i+1}-\boldsymbol{x}_{i}\right)=\mathbf{0}
$$


where $\boldsymbol{J}\left(\boldsymbol{x}_{i}\right)$ is just another notation for $\nabla \boldsymbol{F}\left(\boldsymbol{x}_{i}\right)$. The equation (6.13) is a linear system with coefficient matrix $\boldsymbol{J}$ and right-hand side vector $\boldsymbol{F}\left(\boldsymbol{x}_{i}\right)$. We therefore write this system in the more familiar form

$$
\boldsymbol{J}\left(\boldsymbol{x}_{i}\right) \boldsymbol{\delta}=-\boldsymbol{F}\left(\boldsymbol{x}_{i}\right),
$$

where we have introduce a symbol $\boldsymbol{\delta}$ for the unknown vector $\boldsymbol{x}_{i+1}-\boldsymbol{x}_{i}$ that multiplies the Jacobian $\boldsymbol{J}$.

The $i$-th iteration of Newton's method for systems of algebraic equations consists of two steps:

1. Solve the linear system $\boldsymbol{J}\left(\boldsymbol{x}_{i}\right) \boldsymbol{\delta}=-\boldsymbol{F}\left(\boldsymbol{x}_{i}\right)$ with respect to $\boldsymbol{\delta}$.

2. Set $\boldsymbol{x}_{i+1}=\boldsymbol{x}_{i}+\boldsymbol{\delta}$.

Solving systems of linear equations must make use of appropriate software. Gaussian elimination is the most common, and in general the most robust, method for this purpose. Matlab interfaces the well-known LAPACK package with high-quality and very well tested subroutines for linear algebra. The backslash operator solves a linear system $A x=b$ by $\mathrm{x}=\mathrm{A} \backslash \mathrm{b}$ by a method based on Gaussian elimination.

When nonlinear systems of algebraic equations arise from discretization of partial differential equations, the Jacobian is very often sparse, i.e., most of its elements are zero. In such cases it is important to use algorithms that can take advantage of the many zeros. Gaussian elimination is then a slow method, and (much) faster methods are based on iterative techniques.

\subsubsection{Implementation}

Here is a very simple implementation of Newton's method for systems of nonlinear algebraic equations:

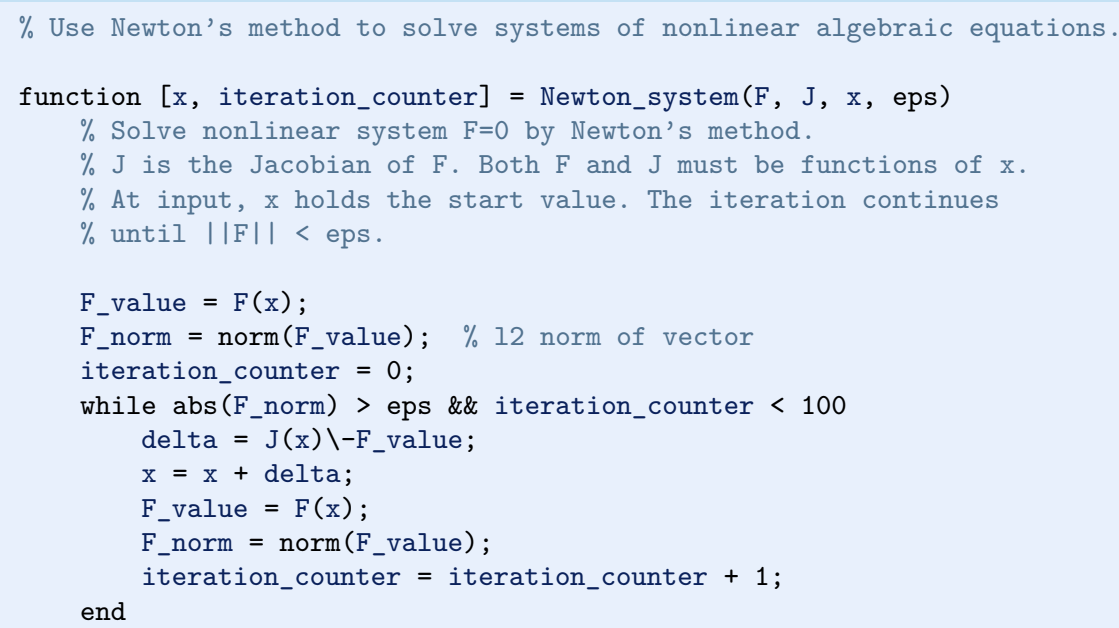


$\%$ Here, either a solution is found, or too many iterations

if abs(F_norm) > eps

end

iteration_counter $=-1$;

end

We can test the function Newton_system with the $2 \times 2$ system (6.11)-(6.12):

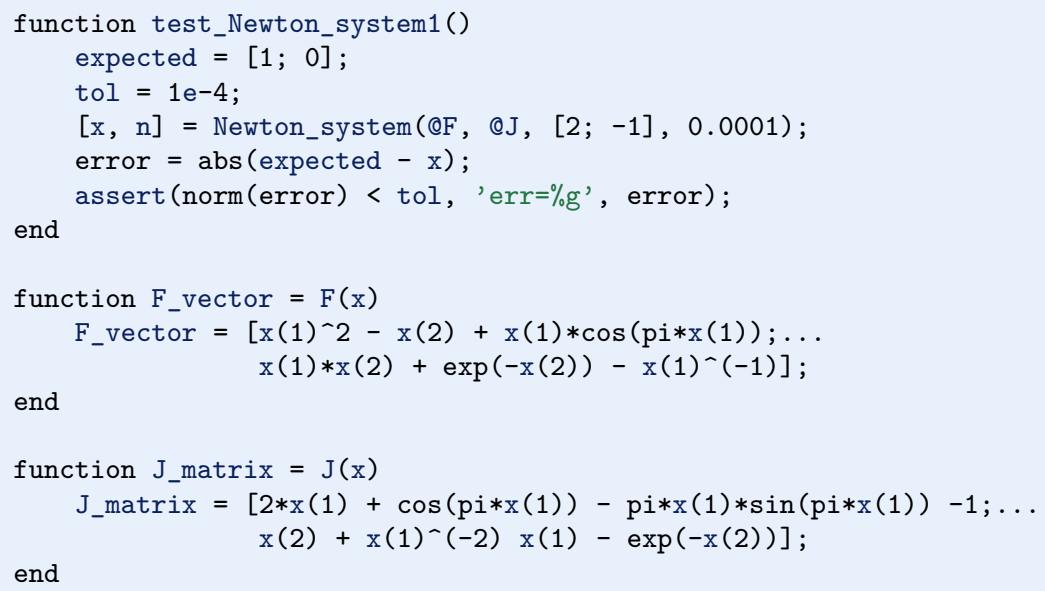

Here, the testing is based on the L2 norm of the error vector. Alternatively, we could test against the values of $\mathrm{x}$ that the algorithm finds, with appropriate tolerances. For example, as chosen for the error norm, if eps $=0.0001$, a tolerance of $10^{-4}$ can be used for $\mathrm{x}[0]$ and $\mathrm{x}[1]$.

\subsection{Exercises}

\section{Exercise 6.1: Understand why Newton's method can fail}

The purpose of this exercise is to understand when Newton's method works and fails. To this end, solve $\tanh x=0$ by Newton's method and study the intermediate details of the algorithm. Start with $x_{0}=1.08$. Plot the tangent in each iteration of Newton's method. Then repeat the calculations and the plotting when $x_{0}=1.09$. Explain what you observe.

Filename: Newton_failure.*.

\section{Exercise 6.2: See if the secant method fails}

Does the secant method behave better than Newton's method in the problem described in Exercise 6.1? Try the initial guesses

1. $x_{0}=1.08$ and $x_{1}=1.09$

2. $x_{0}=1.09$ and $x_{1}=1.1$ 
3. $x_{0}=1$ and $x_{1}=2.3$

4. $x_{0}=1$ and $x_{1}=2.4$

Filename: secant_failure.*.

\section{Exercise 6.3: Understand why the bisection method cannot fail}

Solve the same problem as in Exercise 6.1, using the bisection method, but let the initial interval be $[-5,3]$. Report how the interval containing the solution evolves during the iterations.

Filename: bisection_nonfailure.*.

\section{Exercise 6.4: Combine the bisection method with Newton's method}

An attractive idea is to combine the reliability of the bisection method with the speed of Newton's method. Such a combination is implemented by running the bisection method until we have a narrow interval, and then switch to Newton's method for speed.

Write a function that implements this idea. Start with an interval $[a, b]$ and switch to Newton's method when the current interval in the bisection method is a fraction $s$ of the initial interval (i.e., when the interval has length $s(b-a)$ ). Potential divergence of Newton's method is still an issue, so if the approximate root jumps out of the narrowed interval (where the solution is known to lie), one can switch back to the bisection method. The value of $s$ must be given as an argument to the function, but it may have a default value of 0.1 .

Try the new method on $\tanh (x)=0$ with an initial interval $[-10,15]$.

Filename: bisection_Newton.m.

\section{Exercise 6.5: Write a test function for Newton's method}

The purpose of this function is to verify the implementation of Newton's method in the Newton function in the file Newton.m Construct an algebraic equation and perform two iterations of Newton's method by hand. Find the corresponding size of $|f(x)|$ and use this as value for eps when calling Newton. The function should then also perform two iterations and return the same approximation to the root as you calculated manually. Implement this idea for a unit test as a test function test_Newton().

Filename: test_Newton.m.

\section{Exercise 6.6: Solve nonlinear equation for a vibrating beam}

An important engineering problem that arises in a lot of applications is the vibrations of a clamped beam where the other end is free. This problem can be analyzed analytically, but the calculations boil down to solving the following nonlinear algebraic equation:

$$
\cosh \beta \cos \beta=-1,
$$

where $\beta$ is related to important beam parameters through

$$
\beta^{4}=\omega^{2} \frac{\varrho A}{E I},
$$


where $\varrho$ is the density of the beam, $A$ is the area of the cross section, $E$ is Young's modulus, and $I$ is the moment of the inertia of the cross section. The most important parameter of interest is $\omega$, which is the frequency of the beam. We want to compute the frequencies of a vibrating steel beam with a rectangular cross section having width $b=25 \mathrm{~mm}$ and height $h=8 \mathrm{~mm}$. The density of steel is $7850 \mathrm{~kg} / \mathrm{m}^{3}$, and $E=2 \cdot 10^{11} \mathrm{~Pa}$. The moment of inertia of a rectangular cross section is $I=b h^{3} / 12$.

a) Plot the equation to be solved so that one can inspect where the zero crossings occur.

Hint When writing the equation as $f(\beta)=0$, the $f$ function increases its amplitude dramatically with $\beta$. It is therefore wise to look at an equation with damped amplitude, $g(\beta)=e^{-\beta} f(\beta)=0$. Plot $g$ instead.

b) Compute the first three frequencies.

Filename: beam_vib.m.

Open Access This chapter is distributed under the terms of the Creative Commons AttributionNonCommercial 4.0 International License (http://creativecommons.org/licenses/by-nc/4.0/), which permits any noncommercial use, duplication, adaptation, distribution and reproduction in any medium or format, as long as you give appropriate credit to the original author(s) and the source, a link is provided to the Creative Commons license and any changes made are indicated.

The images or other third party material in this chapter are included in the work's Creative Commons license, unless indicated otherwise in the credit line; if such material is not included in the work's Creative Commons license and the respective action is not permitted by statutory regulation, users will need to obtain permission from the license holder to duplicate, adapt or reproduce the material. 


\section{References}

1. L. Baochuan. Introduction to Numerical Methods. 2015. http://en.wikibooks.org/wiki/ Introduction_to_Numerical_Methods.

2. S. D. Conte and C. de Boor. Elementary Numerical Analysis - An Algorithmic Approach. McGraw-Hill, third edition, 1980.

3. I. Danaila, P. Joly, S. M. Kaber, and M. Postel. An Introduction to Scientific Computing. Springer, 2007.

4. J. W. Eaton, D. Bateman, and S. Hauberg. Gnu octave version 3.0.1 manual: a high-level interactive language for numerical computations. http://www.gnu.org/software/octave/doc/ interpreter/.

5. C. Greif and U. M. Ascher. A First Course in Numerical Methods. Computational Science and Engineering. SIAM, 2011.

6. D. W. Harder and R. Numerical Analysis for Engineering. 2015. https://ece.uwaterloo.ca/ $\sim$ dwharder/NumericalAnalysis/.

7. J. Kiusalaas. Numerical Methods in Engineering with Python. Cambridge, second edition, 2014.

8. H. P. Langtangen. DocOnce publishing platform. https://github.com/hplgit/doconce.

9. H. P. Langtangen. A Primer on Scientific Programming with Python. Texts in Computational Science and Engineering. Springer, fifth edition, 2016.

10. R. LeVeque. Finite Difference Methods for Ordinary and Partial Differential Equations: Steady-State and Time-Dependent Problems. SIAM, 2007.

11. T. Lyche and J.-L. Merrien. Exercises in Computational Mathematics with MATLAB. Springer, 2014.

12. MATLAB software by the mathworks. http://se.mathworks.com/products/matlab/.

13. C. Moler. Numerical Computing with MATLAB. SIAM, 2004. http://se.mathworks.com/moler/ chapters.html.

14. S. Nakamura. Numerical Analysis and Graphic Visualization with Matlab. Prentice Hall, second edition, 2002.

15. S. Otto and J. P. Denier. An Introduction to Programming and Numerical Methods in MATLAB. Springer, 2005.

16. W. H. Press, S. A. Teukolsky, W. T. Vetterling, and B. P. Flannery. Numerical Recipes. Cambridge, 1992. http://www.nrbook.com/a/bookcpdf.php.

17. G. Recktenwald. Numerical Methods with MATLAB: Implementations and Applications. Prentice-Hall, 2000. http://web.cecs.pdx.edu/ gerry/nmm/.

18. G. Sewell. The Numerical Solution of Ordinary and Partial Differential Equations. Wiley, 2005.

(C) The Author(s) 2016

S. Linge, H.P. Langtangen, Programming for Computations - MATLAB/Octave,

Texts in Computational Science and Engineering 14, DOI 10.1007/978-3-319-32452-4 
19. T. Siauw and A. Bayen. An Introduction to MATLAB Programming and Numerical Methods for Engineers. Academic Press, 2014. http://www.sciencedirect.com/science/book/ 9780124202283.

20. L. N. Trefethen. Spectral Methods in MATLAB. SIAM, 2000.

21. L. N. Trefethen. Approximation Theory and Approximation Practice. SIAM, 2012.

22. T. Young and M. J. Mohlenkamp. Introduction to Numerical Methods and MATLAB Programming for Engineers. 2015. https://www.math.ohiou.edu/courses/math3600/book.pdf. 


\section{Index}

2nd-order Runge-Kutta method, 122

\section{A}

algorithm, 3

allocate, 14

anonymous function, 32

argument, 27

array, 9,14

element, 14

index, 14

slice of, 15

sorting, 41

assert (function), 64

assignment, 5

atan, 8

axis (plot), 17

B

boolean, 25

expression, 25

false (0), 25

true (1), 25

boundary conditions, 154

brute force method, 178

bug, 2, 3, 60

C

C, 2

$\mathrm{C}++, 2$

calculator, 5

cell, 156

code, 4

exception, 188

re-use, 54,71

robust, 186

try-catch, 188

comment, 4

commenting code, 21

compartment model, 102

composite midpoint method, 57 composite trapezoidal rule, 49

computational speed (measuring), 69

computer program, 1

convergence rate, 61

copy, 15

Crank-Nicolson method, 132, 147

\section{D}

debugger, 18

debugging, 2, 3, 18

default, 11,12

demo function, 97

difference

absolute, 63

backward, 121

centered, 122

forward, 92, 121

relative, 63

differential equation

first-order, 88

second-order, 116

diffusion equation, 153

discontinuous coefficient, 114

divergence, 186

doc string, 30

DocOnce, ix

domain, 69, 74, 76, 154

complex, 76

double (precision), 11

double integral

midpoint, 69

double sum, 71

dynamical system, 87

E

else, 25

elseif, 25

Emacs, 6

end, 25

error 


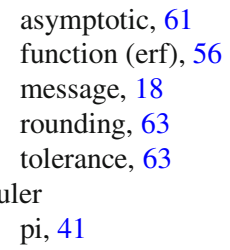

Euler's method, 93 exception handling, 18 execute (a program), 3 exp math notation, 90

\section{F}

false (0), 25

fast code, 21

finite difference method, 91

finite precision (of float), 62

flat program, 54

float, 11

floating point number (float), 63

for loop, 32

format

png, 17

Fortran, 2

forward difference approximation, 92

Forward Euler scheme, 93

Fourier series, 44

function, 8, 27

anonymous, 32

assert, 64

call, 8

definition, 27

global, 32

handle, 31

input parameter, 8

local, 32

nargin, 30

nargout, 30

nested, 32

output parameter, 8

return, 8

take a parameter, 8

\section{G}

Gauss quadrature, 59

Gedit, 6

graph, 15

\section{H}

hardcopy (plot), 17

heat equation, 153

Heun's method, 122

hold (on/off), 15

\section{I}

if, 25

implement (a program), 3 implementation

general, 52

specific, 52

indexing

one based, 14

zero based, 14

initial conditions, 154

input, 19

instability, 162

instruction, 4

integer, 11

integral

analytically, 47

approximately, 47

exact, 47

numerically, 47

integration

points, 49

interactive use (of Matlab), 10

$\mathbf{K}$

keyboard

arrow up/down, 10

L

language

computer, 2

programming, 2

Laplace equation, 174

least squares method, 43

legend (plot), 17

Leibniz

pi, 41

linear algebra, 17, 34

linear interpolation, 42

linspace, 8

logistic model

carrying capacity, 99

long lines (splitting of), 21

loop

double, 34

for, 32

index, 32, 35

infinite, 35

iteration, 32, 35

multiple, 34

nested, 34

while, 35

\section{M}

main program, 28

Maple, 2

Mathematica, 2, 20

mathematical modeling, 108

MATLAB, 2

Matlab

documentation, 21 
indent, 21

licence, 21

one-based indexing, 15

matrix, 17

tridiagonal, 169

vector product, 17

mesh, 91

points, 91,156

uniform, 91

method of lines, 155, 156

m-file, 7

Midpoint method, 57

model

computational, 89

differential equation, 88

mathematical, 3, 88

MOL, 155

forward Euler, 155

Monte Carlo integration, 76

\section{N}

Newton

starting value, 188

nonlinear algebraic equation, 123

Notepad++, 6

numerical scheme, 93

O

Octave, 2

ODE

scalar, 108

vector, 108

ode $23 \mathrm{~s}, 123$

ode 45,123

operator

Arithmetic, 11

Logical, 26

\section{$\mathbf{P}$}

parameter

input, 27

output, 27

parentheses, 11

PDE, 153

plot, 8,9

figure, 16

Poisson equation, 174

print, 3

printf formatting, 12

printing

formatted, 12

program

crash, 18

execute, 4,6

flat, 54

input, 19

output, 19 run, 4, 6

statement, 4

testing, 18

typing, 6

verification, 18

programming, 2

game, 42

prompt, 6, 10

pseudo code, 25

Python, 2

\section{R}

rand (function), 26

random walk, 25

rate of convergence, 61, 193

read (from file), 36

reserved words, 11

resonance, 138

return, 27

None, 193

value, 29

RK2, 122

root finding, 178

rounding error, 11

Runge-Kutta, 2nd-order method, 122

Runge-Kutta-Fehlberg, 128

\section{S}

Sage (symbolic package), 21

scalar ODE, 108

scaling, 136, 163

scheme, 88

script (and scripting), 3

second-order ODE rewritten as two first-order ODEs, 117

seed (random generators), 79

semi-colon (easy print), 5

simple pendulum, 116

Simpson's rule, 59

simulation, 3

single (precision), 11

SIR model, 102

source term, 153

spring

damping of, 115, 133

linear, 136

nonlinear, 133

oscillations, 115

stability criterion, 162

stop program $(\mathrm{Ctrl}+\mathrm{c}), 36$

string, 11

symbolic

computations, 19

operations, 19

simplifications, 19

Symbolic Toolbox, 19

syntax, 2 
system of ODEs, 108

T

Taylor series, 148

test function, 64

testing, 18

testing procedures, 61

text editor, 6

TextWrangler, 6

theta rule, 171

title (plot), 17

Trapezoidal rule, 49

tridiagonal matrix, 169

triple integral

midpoint, 73

true (1), 25

try-catch, 18

type conversion, 11

U

unit tests, 60

unstable solutions, 162

V

validation, 18

variable, 5

assignment, 11

delete, 21 float, 11

global, 30

integer, 11

local, 30

name, 11

string, 11

type, 11

vector, 17

vector ODE, 108

vectorization, 67, 165

verification, 18

Verlet integration, 142

Vim, 6

W

while loop, 35

WolframAlpha, 20

write (to file), 36

\section{X}

xlabel, 9

Y

ylabel, 9

Z

zeros, 14 


\section{Editorial Policy}

$\S 1$. Textbooks on topics in the field of computational science and engineering will be considered. They should be written for courses in CSE education. Both graduate and undergraduate textbooks will be published in TCSE. Multidisciplinary topics and multidisciplinary teams of authors are especially welcome.

§2. Format: Only works in English will be considered. For evaluation purposes, manuscripts may be submitted in print or electronic form, in the latter case, preferably as pdf- or zipped ps-files. Authors are requested to use the LaTeX style files available from Springer at: https://www.springer.com/gp/authors-editors/bookauthors-editors/manuscript-preparation/5636 (Click on $\longrightarrow$ Templates $\longrightarrow$ LaTeX $\longrightarrow$ monographs)

Electronic material can be included if appropriate. Please contact the publisher.

$\S 3$. Those considering a book which might be suitable for the series are strongly advised to contact the publisher or the series editors at an early stage.

\section{General Remarks}

Careful preparation of manuscripts will help keep production time short and ensure a satisfactory appearance of the finished book.

The following terms and conditions hold:

Regarding free copies and royalties, the standard terms for Springer mathematics textbooks hold. Please write to martin.peters@springer.com for details.

Authors are entitled to purchase further copies of their book and other Springer books for their personal use, at a discount of $33.3 \%$ directly from Springer-Verlag. 
Timothy J. Barth

NASA Ames Research Center

NAS Division

Moffett Field, CA 94035, USA

barth@nas.nasa.gov

Michael Griebel

Institut für Numerische Simulation

der Universität Bonn

Wegelerstr. 6

53115 Bonn, Germany

griebel@ins.uni-bonn.de

David E. Keyes

Mathematical and Computer Sciences and Engineering

King Abdullah University of Science and Technology

P.O. Box 55455

Jeddah 21534, Saudi Arabia

david.keyes@kaust.edu.sa

and

Department of Applied Physics and Applied Mathematics

Columbia University

500 W. 120 th Street

New York, NY 10027, USA

kd2112@columbia.edu
Risto M. Nieminen

Department of Applied Physics

Aalto University School of Science

and Technology

00076 Aalto, Finland

risto.nieminen@aalto.fi

Dirk Roose

Department of Computer Science

Katholieke Universiteit Leuven

Celestijnenlaan 200A

3001 Leuven-Heverlee, Belgium

dirk.roose@cs.kuleuven.be

Tamar Schlick

Department of Chemistry

and Courant Institute

of Mathematical Sciences

New York University

251 Mercer Street

New York, NY 10012, USA

schlick@nyu.edu

Editor for Computational Science and Engineering at Springer:

Martin Peters

Springer-Verlag

Mathematics Editorial IV

Tiergartenstrasse 17

69121 Heidelberg, Germany

martin.peters@springer.com 


\section{Texts in Computational Science and Engineering}

1. H. P. Langtangen, Computational Partial Differential Equations. Numerical Methods and Diffpack Programming. 2nd Edition

2. A. Quarteroni, F. Saleri, P. Gervasio, Scientific Computing with MATLAB and Octave. 4th Edition

3. H. P. Langtangen, Python Scripting for Computational Science. 3rd Edition

4. H. Gardner, G. Manduchi, Design Patterns for e-Science.

5. M. Griebel, S. Knapek, G. Zumbusch, Numerical Simulation in Molecular Dynamics.

6. H. P. Langtangen, A Primer on Scientific Programming with Python. 5th Edition

7. A. Tveito, H. P. Langtangen, B. F. Nielsen, X. Cai, Elements of Scientific Computing.

8. B. Gustafsson, Fundamentals of Scientific Computing.

9. M. Bader, Space-Filling Curves.

10. M. Larson, F. Bengzon, The Finite Element Method: Theory, Implementation and Applications.

11. W. Gander, M. Gander, F. Kwok, Scientific Computing: An Introduction using Maple and MATLAB.

12. P. Deuflhard, S. Röblitz, A Guide to Numerical Modelling in Systems Biology.

13. M. H. Holmes, Introduction to Scientific Computing and Data Analysis.

14. S. Linge, H. P. Langtangen, Programming for Computations - A Gentle Introduction to Numerical Simulations with MATLAB/Octave.

15. S. Linge, H. P. Langtangen, Programming for Computations - A Gentle Introduction to Numerical Simulations with Python.

For further information on these books please have a look at our mathematics catalogue at the following URL: www.springer.com/series/5151

\section{Monographs in Computational Science and Engineering}

1. J. Sundnes, G.T. Lines, X. Cai, B.F. Nielsen, K.-A. Mardal, A. Tveito, Computing the Electrical Activity in the Heart.

For further information on this book, please have a look at our mathematics catalogue at the following URL: www.springer.com/series/7417 


\section{Lecture Notes \\ in Computational Science \\ and Engineering}

1. D. Funaro, Spectral Elements for Transport-Dominated Equations.

2. H.P. Langtangen, Computational Partial Differential Equations. Numerical Methods and Diffpack Programming.

3. W. Hackbusch, G. Wittum (eds.), Multigrid Methods V.

4. P. Deuflhard, J. Hermans, B. Leimkuhler, A.E. Mark, S. Reich, R.D. Skeel (eds.), Computational Molecular Dynamics: Challenges, Methods, Ideas.

5. D. Kröner, M. Ohlberger, C. Rohde (eds.), An Introduction to Recent Developments in Theory and Numerics for Conservation Laws.

6. S. Turek, Efficient Solvers for Incompressible Flow Problems. An Algorithmic and Computational Approach.

7. R. von Schwerin, Multi Body System SIMulation. Numerical Methods, Algorithms, and Software.

8. H.-J. Bungartz, F. Durst, C. Zenger (eds.), High Performance Scientific and Engineering Computing.

9. T.J. Barth, H. Deconinck (eds.), High-Order Methods for Computational Physics.

10. H.P. Langtangen, A.M. Bruaset, E. Quak (eds.), Advances in Software Tools for Scientific Computing.

11. B. Cockburn, G.E. Karniadakis, C.-W. Shu (eds.), Discontinuous Galerkin Methods. Theory, Computation and Applications.

12. U. van Rienen, Numerical Methods in Computational Electrodynamics. Linear Systems in Practical Applications.

13. B. Engquist, L. Johnsson, M. Hammill, F. Short (eds.), Simulation and Visualization on the Grid.

14. E. Dick, K. Riemslagh, J. Vierendeels (eds.), Multigrid Methods VI.

15. A. Frommer, T. Lippert, B. Medeke, K. Schilling (eds.), Numerical Challenges in Lattice Quantum Chromodynamics.

16. J. Lang, Adaptive Multilevel Solution of Nonlinear Parabolic PDE Systems. Theory, Algorithm, and Applications.

17. B.I. Wohlmuth, Discretization Methods and Iterative Solvers Based on Domain Decomposition.

18. U. van Rienen, M. Günther, D. Hecht (eds.), Scientific Computing in Electrical Engineering.

19. I. Babuška, P.G. Ciarlet, T. Miyoshi (eds.), Mathematical Modeling and Numerical Simulation in Continuum Mechanics.

20. T.J. Barth, T. Chan, R. Haimes (eds.), Multiscale and Multiresolution Methods. Theory and Applications.

21. M. Breuer, F. Durst, C. Zenger (eds.), High Performance Scientific and Engineering Computing.

22. K. Urban, Wavelets in Numerical Simulation. Problem Adapted Construction and Applications.

23. L.F. Pavarino, A. Toselli (eds.), Recent Developments in Domain Decomposition Methods.

24. T. Schlick, H.H. Gan (eds.), Computational Methods for Macromolecules: Challenges and Applications. 
25. T.J. Barth, H. Deconinck (eds.), Error Estimation and Adaptive Discretization Methods in Computational Fluid Dynamics.

26. M. Griebel, M.A. Schweitzer (eds.), Meshfree Methods for Partial Differential Equations.

27. S. Müller, Adaptive Multiscale Schemes for Conservation Laws.

28. C. Carstensen, S. Funken, W. Hackbusch, R.H.W. Hoppe, P. Monk (eds.), Computational Electromagnetics.

29. M.A. Schweitzer, A Parallel Multilevel Partition of Unity Method for Elliptic Partial Differential Equations.

30. T. Biegler, O. Ghattas, M. Heinkenschloss, B. van Bloemen Waanders (eds.), Large-Scale PDEConstrained Optimization.

31. M. Ainsworth, P. Davies, D. Duncan, P. Martin, B. Rynne (eds.), Topics in Computational Wave Propagation. Direct and Inverse Problems.

32. H. Emmerich, B. Nestler, M. Schreckenberg (eds.), Interface and Transport Dynamics. Computational Modelling.

33. H.P. Langtangen, A. Tveito (eds.), Advanced Topics in Computational Partial Differential Equations. Numerical Methods and Diffpack Programming.

34. V. John, Large Eddy Simulation of Turbulent Incompressible Flows. Analytical and Numerical Results for a Class of LES Models.

35. E. Bänsch (ed.), Challenges in Scientific Computing - CISC 2002.

36. B.N. Khoromskij, G. Wittum, Numerical Solution of Elliptic Differential Equations by Reduction to the Interface.

37. A. Iske, Multiresolution Methods in Scattered Data Modelling.

38. S.-I. Niculescu, K. Gu (eds.), Advances in Time-Delay Systems.

39. S. Attinger, P. Koumoutsakos (eds.), Multiscale Modelling and Simulation.

40. R. Kornhuber, R. Hoppe, J. Périaux, O. Pironneau, O. Wildlund, J. Xu (eds.), Domain Decomposition Methods in Science and Engineering.

41. T. Plewa, T. Linde, V.G. Weirs (eds.), Adaptive Mesh Refinement - Theory and Applications.

42. A. Schmidt, K.G. Siebert, Design of Adaptive Finite Element Software. The Finite Element Toolbox ALBERTA.

43. M. Griebel, M.A. Schweitzer (eds.), Meshfree Methods for Partial Differential Equations II.

44. B. Engquist, P. Lötstedt, O. Runborg (eds.), Multiscale Methods in Science and Engineering.

45. P. Benner, V. Mehrmann, D.C. Sorensen (eds.), Dimension Reduction of Large-Scale Systems.

46. D. Kressner, Numerical Methods for General and Structured Eigenvalue Problems.

47. A. Boriçi, A. Frommer, B. Joó, A. Kennedy, B. Pendleton (eds.), QCD and Numerical Analysis III.

48. F. Graziani (ed.), Computational Methods in Transport.

49. B. Leimkuhler, C. Chipot, R. Elber, A. Laaksonen, A. Mark, T. Schlick, C. Schütte, R. Skeel (eds.), New Algorithms for Macromolecular Simulation.

50. M. Bücker, G. Corliss, P. Hovland, U. Naumann, B. Norris (eds.), Automatic Differentiation: Applications, Theory, and Implementations.

51. A.M. Bruaset, A. Tveito (eds.), Numerical Solution of Partial Differential Equations on Parallel Computers.

52. K.H. Hoffmann, A. Meyer (eds.), Parallel Algorithms and Cluster Computing. 
53. H.-J. Bungartz, M. Schäfer (eds.), Fluid-Structure Interaction.

54. J. Behrens, Adaptive Atmospheric Modeling.

55. O. Widlund, D. Keyes (eds.), Domain Decomposition Methods in Science and Engineering XVI.

56. S. Kassinos, C. Langer, G. Iaccarino, P. Moin (eds.), Complex Effects in Large Eddy Simulations.

57. M. Griebel, M.A Schweitzer (eds.), Meshfree Methods for Partial Differential Equations III.

58. A.N. Gorban, B. Kégl, D.C. Wunsch, A. Zinovyev (eds.), Principal Manifolds for Data Visualization and Dimension Reduction.

59. H. Ammari (ed.), Modeling and Computations in Electromagnetics: A Volume Dedicated to JeanClaude Nédélec.

60. U. Langer, M. Discacciati, D. Keyes, O. Widlund, W. Zulehner (eds.), Domain Decomposition Methods in Science and Engineering XVII.

61. T. Mathew, Domain Decomposition Methods for the Numerical Solution of Partial Differential Equations.

62. F. Graziani (ed.), Computational Methods in Transport: Verification and Validation.

63. M. Bebendorf, Hierarchical Matrices. A Means to Efficiently Solve Elliptic Boundary Value Problems.

64. C.H. Bischof, H.M. Bücker, P. Hovland, U. Naumann, J. Utke (eds.), Advances in Automatic Differentiation.

65. M. Griebel, M.A. Schweitzer (eds.), Meshfree Methods for Partial Differential Equations IV.

66. B. Engquist, P. Lötstedt, O. Runborg (eds.), Multiscale Modeling and Simulation in Science.

67. I.H. Tuncer, Ü. Gülcat, D.R. Emerson, K. Matsuno (eds.), Parallel Computational Fluid Dynamics 2007.

68. S. Yip, T. Diaz de la Rubia (eds.), Scientific Modeling and Simulations.

69. A. Hegarty, N. Kopteva, E. O'Riordan, M. Stynes (eds.), BAIL 2008 - Boundary and Interior Layers.

70. M. Bercovier, M.J. Gander, R. Kornhuber, O. Widlund (eds.), Domain Decomposition Methods in Science and Engineering XVIII.

71. B. Koren, C. Vuik (eds.), Advanced Computational Methods in Science and Engineering.

72. M. Peters (ed.), Computational Fluid Dynamics for Sport Simulation.

73. H.-J. Bungartz, M. Mehl, M. Schäfer (eds.), Fluid Structure Interaction II - Modelling, Simulation, Optimization.

74. D. Tromeur-Dervout, G. Brenner, D.R. Emerson, J. Erhel (eds.), Parallel Computational Fluid Dynamics 2008.

75. A.N. Gorban, D. Roose (eds.), Coping with Complexity: Model Reduction and Data Analysis.

76. J.S. Hesthaven, E.M. Rønquist (eds.), Spectral and High Order Methods for Partial Differential Equations.

77. M. Holtz, Sparse Grid Quadrature in High Dimensions with Applications in Finance and Insurance.

78. Y. Huang, R. Kornhuber, O.Widlund, J. Xu (eds.), Domain Decomposition Methods in Science and Engineering XIX.

79. M. Griebel, M.A. Schweitzer (eds.), Meshfree Methods for Partial Differential Equations V.

80. P.H. Lauritzen, C. Jablonowski, M.A. Taylor, R.D. Nair (eds.), Numerical Techniques for Global Atmospheric Models. 
81. C. Clavero, J.L. Gracia, F.J. Lisbona (eds.), BAIL 2010 - Boundary and Interior Layers, Computational and Asymptotic Methods.

82. B. Engquist, O. Runborg, Y.R. Tsai (eds.), Numerical Analysis and Multiscale Computations.

83. I.G. Graham, T.Y. Hou, O. Lakkis, R. Scheichl (eds.), Numerical Analysis of Multiscale Problems.

84. A. Logg, K.-A. Mardal, G. Wells (eds.), Automated Solution of Differential Equations by the Finite Element Method.

85. J. Blowey, M. Jensen (eds.), Frontiers in Numerical Analysis - Durham 2010.

86. O. Kolditz, U.-J. Gorke, H. Shao, W. Wang (eds.), Thermo-Hydro-Mechanical-Chemical Processes in Fractured Porous Media - Benchmarks and Examples.

87. S. Forth, P. Hovland, E. Phipps, J. Utke, A. Walther (eds.), Recent Advances in Algorithmic Differentiation.

88. J. Garcke, M. Griebel (eds.), Sparse Grids and Applications.

89. M. Griebel, M.A. Schweitzer (eds.), Meshfree Methods for Partial Differential Equations VI.

90. C. Pechstein, Finite and Boundary Element Tearing and Interconnecting Solvers for Multiscale Problems.

91. R. Bank, M. Holst, O. Widlund, J. Xu (eds.), Domain Decomposition Methods in Science and Engineering $X X$.

92. H. Bij1, D. Lucor, S. Mishra, C. Schwab (eds.), Uncertainty Quantification in Computational Fluid Dynamics.

93. M. Bader, H.-J. Bungartz, T. Weinzierl (eds.), Advanced Computing.

94. M. Ehrhardt, T. Koprucki (eds.), Advanced Mathematical Models and Numerical Techniques for Multi-Band Effective Mass Approximations.

95. M. Azaïez, H. El Fekih, J.S. Hesthaven (eds.), Spectral and High Order Methods for Partial Differential Equations ICOSAHOM 2012.

96. F. Graziani, M.P. Desjarlais, R. Redmer, S.B. Trickey (eds.), Frontiers and Challenges in Warm Dense Matter.

97. J. Garcke, D. Pflüger (eds.), Sparse Grids and Applications - Munich 2012.

98. J. Erhel, M. Gander, L. Halpern, G. Pichot, T. Sassi, O. Widlund (eds.), Domain Decomposition Methods in Science and Engineering XXI.

99. R. Abgrall, H. Beaugendre, P.M. Congedo, C. Dobrzynski, V. Perrier, M. Ricchiuto (eds.), High Order Nonlinear Numerical Methods for Evolutionary PDEs - HONOM 2013.

100. M. Griebel, M.A. Schweitzer (eds.), Meshfree Methods for Partial Differential Equations VII.

101. R. Hoppe (ed.), Optimization with PDE Constraints - OPTPDE 2014.

102. S. Dahlke, W. Dahmen, M. Griebel, W. Hackbusch, K. Ritter, R. Schneider, C. Schwab, H. Yserentant (eds.), Extraction of Quantifiable Information from Complex Systems.

103. A. Abdulle, S. Deparis, D. Kressner, F. Nobile, M. Picasso (eds.), Numerical Mathematics and Advanced Applications - ENUMATH 2013.

104. T. Dickopf, M.J. Gander, L. Halpern, R. Krause, L.F. Pavarino (eds.), Domain Decomposition Methods in Science and Engineering XXII.

105. M. Mehl, M. Bischoff, M. Schäfer (eds.), Recent Trends in Computational Engineering - CE2014. Optimization, Uncertainty, Parallel Algorithms, Coupled and Complex Problems.

106. R.M. Kirby, M. Berzins, J.S. Hesthaven (eds.), Spectral and High Order Methods for Partial Differential Equations - ICOSAHOM'14. 
107. B. Jüttler, B. Simeon (eds.), Isogeometric Analysis and Applications 2014.

108. P. Knobloch (ed.), Boundary and Interior Layers, Computational and Asymptotic Methods - BAIL 2014.

109. J. Garcke, D. Pflüger (eds.), Sparse Grids and Applications - Stuttgart 2014.

110. H.P. Langtangen, Finite Difference Computing with Exponential Decay Models.

111. A. Tveito, G.T. Lines, Computing Characterizations of Drugs for Ion Channels and Receptors Using Markov Models

For further information on these books please have a look at our mathematics catalogue at the following URL: www.springer.com/series/3527 